\title{
Depositionsmechanismen, Struktur und mechanische Eigenschaften laserdeponierter Poly(methyl methacrylat)-Filme
}

\author{
Dissertation \\ zur Erlangung des Doktorgrades \\ der Mathematisch-Naturwissenschaftlichen Fakultäten \\ der Georg-August-Universität zu Göttingen
}

Vorgelegt von

Thorsten Scharf

aus Dinkelsbühl

Göttingen 2006 
D7

Referent: $\quad$ Prof. Dr. H.-U. Krebs

Korreferent: Prof. Dr. M. Buback

Tag der mündlichen Prüfung: 


\section{Inhaltsverzeichnis}

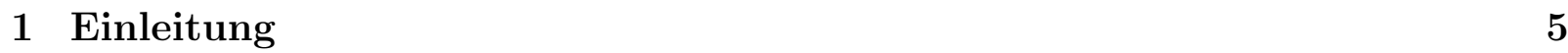

\begin{tabular}{|lrr}
\hline 2 & Experimentelle Methoden & 9
\end{tabular}

2.1 Gepulste Laserdeposition (PLD) . . . . . . . . . . . . . . . . . . . . . . 9

2.1 .1 Verwendeter Aufbau . . . . . . . . . . . . . . . . . . . 10

2.1 .2 Polymertargets . . . . . . . . . . . . . . . . . . . 11

$2.1 .3 \mathrm{MAPLE} \ldots \ldots \ldots \ldots \ldots \ldots$

2.2 Charakterisierungsmethoden . . . . . . . . . . . . . . . . . 16

2.2 .1 Plasma Puls angeregtes Reed (PPXR) . . . . . . . . . . . . 16

2.2 .2 Doppelpaddeloszillator (DPO) . . . . . . . . . . . . . 16

$2.2 .3 \quad$ Dielektrische Spektroskopie (DS) . . . . . . . . . . . . . . . . 17

$2.2 .4 \quad$ Fourier Transformations IR-Spektroskopie (FTIR) . . . . . . . . . . 17

2.2 .5 Größenausschlusschromatographie (SEC) . . . . . . . . . . . . 17

2.2 .6 Rasterelektronenmikroskopie (SEM) . . . . . . . . . . . . . . . 18

2.2 .7 Rasterkraftmikroskopie $(\mathrm{AFM})$. . . . . . . . . . . . . . . . 18

2.2 .8 Röntgenreflektometrie (XRR) . . . . . . . . . . . . . . . 18

3 Entwicklung des Plasma Puls angeregten Reed (PPXR) 19

3.1 Idee $\ldots \ldots \ldots \ldots \ldots$

3.2 Beschreibung der Schwingung . . . . . . . . . . . . . . . . . 20

3.2 .1 Balkenbiegung . . . . . . . . . . . . . . . . 20

3.2 .2 Auslenkung durch das Plasma . . . . . . . . . . . . . . . . . . 25

3.3 Mechanischer Aufbau der Apparatur . . . . . . . . . . . . . . . . . . . . . 28

3.4 Elektronische Messwerterfassung . . . . . . . . . . . . . . . . . . . . . . 30

3.5 Ablauf des Messverfahrens . . . . . . . . . . . . . . . . . . . . . . . . . 31

3.6 Auswertung der Messung . . . . . . . . . . . . . . . . . . . . . . 32

$3.6 .1 \quad$ Alternative Auswertung im Fourier-Raum . . . . . . . . . . . . . . 34

3.7 Berechnung des komplexen Elastizitäts-Moduls . . . . . . . . . . . . . . . . 36 
4 Gepulste Laserdeposition von PMMA $\quad 41$

4.1 Inkubation . . . . . . . . . . . . . . . . . . . . . 43

4.2 Laser-Ablation von PMMA . . . . . . . . . . . . . . . . . . . . . . . . . . 47

4.2 .1 Kleine Fragmente . . . . . . . . . . . . . . . . . . . . . . . . 47

4.2 .2 Droplets . . . . . . . . . . . . . . . . . . . . . . . . . . . 49

4.3 Deposition . . . . . . . . . . . . . . . . . . . . 50

$4.3 .1 \quad$ Glatter Filmanteil . . . . . . . . . . . . . . . . . . . . . . . . 51

4.3 .2 Droplets . . . . . . . . . . . . . . . . . . 52

4.4 Zusammenfassung . . . . . . . . . . . . . . . . . 57

5 Modifikation der gepulsten Laserdepositon durch Targetbeigaben 59

5.1 Antracen . . . . . . . . . . . . . . . . . . 59

5.2 UV absorbierender RAFT-Agent . . . . . . . . . . . . . . . . . . . 68

5.3 Copolymere . . . . . . . . . . . . . . . . . . 73

5.4 MAPLE . . . . . . . . . . . . . . . . . . . . . . 79

5.4 .1 Dimethoxyethan als Matrix . . . . . . . . . . . . . . . . . 79

$5.4 .2 \quad$ DME-Matrix unter Inertgasatmosphäre . . . . . . . . . . . . . . . . 83

5.4 .3 Toluol als Matrix . . . . . . . . . . . . . . . . . . . . . . . . . 84

6 Mechanische Eigenschaften von laserdeponiertem PMMA 91

6.1 Grundlagen . . . . . . . . . . . . . . . . . . . . 91

6.1 .1 Qualitative Befunde . . . . . . . . . . . . . . . 91

6.1 .2 Beobachtungen in der Zeitdomäne . . . . . . . . . . . . . . . . . . . 94

6.1 .3 Temperaturabhängigkeit . . . . . . . . . . . . . . . . . . 95

6.1 .4 Übergang zur periodischen Anregung . . . . . . . . . . . . . . . . . 96

6.2 Komplexer Elastizitätsmodul. . . . . . . . . . . . . . . . . . . . . . . . . . 97

6.3 Thermische Behandlung von PMMA . . . . . . . . . . . . . . . . . . . . 103

6.4 Vergleich spektroskopischer Methoden . . . . . . . . . . . . . . . . . . . . 106

6.5 Abhängigkeit der Messung von der Resonanzfrequenz . . . . . . . . . . . . 112

6.6 Modifikation der Filme durch die Substrattemperatur . . . . . . . . . . . . 113

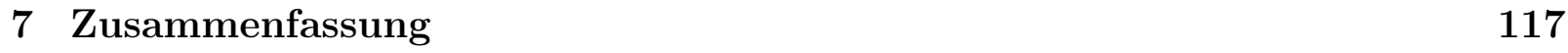

\begin{tabular}{lr}
\hline Literaturverzeichnis & 121
\end{tabular} 


\section{Einleitung}

Während unsere tägliche Umgebung scheinbar vielfach von anorganischen Werkstoffen dominiert wird, sind organische Materialien, vor allem in Form von Polymeren, aus unserem Alltag nicht mehr wegzudenken. Sie zeichnen sich gegenüber anderen Materialien durch eine Vielzahl von Vorteilen aus, wie die geringe Dichte, einfache Verarbeitbarkeit, hohe Korrosionsbeständigkeit und auch den meist niedrigen Preis. Sie werden für eine unüberschaubare Breite von Anwedungen benutzt, angefangen von einfachen Verpackungen über Textilien und Autoreifen bis hin zu Lacken und zu Komponenten für die Mikroelektronik. Dabei sind Polymere nicht nur synthetisch hergestellt, beispielsweise Zellulose oder auch DNA sind natürliche Polymere. In der Anwendung als Werkstoffe dominieren jedoch die synthetischen Polymere [53].

Von zusätzlichem Interesse für die Anwendung sind Kompositmaterialien, die aus der Verbindung verschiedener Materialien aufgebaut sind. In diesen lassen sich bei geschickter Kombination die positiven Eigenschaften mehrerer Werkstoffe vereinen [56]. Eine besondere Herausforderung der Herstellung mikroskopischer Verbundmaterialien ist meist dann gegeben, wenn verschiedene Materialklassen miteinander vereinigt werden sollen, da diese häufig völlig unterschiedliche, teilweise inkompatible Prozesse erfordern.

Auch dünne Filme organischer Materialien gewinnen in den letzten Jahren immer mehr an Bedeutung. Vor allem die fortschreitende Miniaturisierung im Bereich der Mikroelektronik, aber auch optische, katalytische, mikrosensorische und pharmazeutische Anwendungen spielen eine wachsende Rolle [19]. Standardmäßig werden diese durch nasschemische Verfahren wie spin coating hergestellt, was eine gut reproduzierbare chemische Struktur und gut einstellbare Eigenschaften des Films ermöglicht. Sind diese Verfahren wegen der benötigten Lösungsmittel nicht einsetzbar, so wird meist die Plasmapolymerisation verwendet [54], die jedoch nur für wenige Polymere genutzt werden kann und chemisch stark vernetzte Polymere produziert.

Als eine flexible Methode zur Herstellung dünner Schichten vieler Materialklassen wie Keramiken [131, 39], Halbleiter [136] und Metalle [43] hat sich die gepulste Laserdeposition (Pulsed Laser Deposition, PLD) in den letzen Jahrzehnten etabliert [29, 18, 62, 135]. Mit ihr können Kompositmaterialien in Form von homogenen Legierungen [60], inhomogenen Legierungen inklusive Nanoclustern [36, 91] bis hin zu Schichtpaketen [39] deponiert wer- 
den. Weiterhin ist dieses Verfahren frei von Lösungsmitteln und die Schichteigenschaften lassen sich in einem weiten Bereich variieren. Auch die Deposition von organischen Materialien in Form von Polymeren wird seit längerer Zeit untersucht [46, 45] und stellt sich erwartungsgemäß als schwierig heraus. Mit der üblichen Deposition mit UV-Lasern lassen sich nur ausgewählte Polymere deponieren [116] und auch diese zeigen vielfach eine stark veränderte chemische Struktur und eine unerwünschte Filmmorphologie [120]. Die Depositon mittels IR-Lasern, deren Wellenlänge an ausgewählte Molekülschwingungen angepasst wurde, wurde erfolgreich demonstriert [13, 14], erfordert jedoch einen Freie-ElektronenLaser, was die meisten praktischen Anwendungen ausschließt.

Das für die gepulste Laserdeposition im UV-Bereich am besten untersuchte Polymer und damit ideale Modellsystem für die Untersuchung von organischen Materialien, ist Poly(methyl methacrylat) (PMMA) [108]. Dieses ist ein transparenter, weitgehend amorpher Thermoplast, der unter dem Handelsnamen Plexiglas bekannt ist. PMMA wird neben der Hauptanwendung in Fensterscheiben auch in dünnen Schichten unter anderem als Photoresist und Schutzschicht gegen Elektronenstrahlung eingesetzt.

Aus vorhergehenden Arbeiten, unter anderem von Süske [117], ist bekannt, dass laserdeponierte PMMA Filme aus zwei Komponenten bestehen, einem glatten Filmanteil, der hauptsächlich aus hochvernetztem PMMA besteht, und überlagert einem Anteil aus Tröpfchen mit einigen $\mu \mathrm{m}$ Durchmesser, die aus PMMA mit stark reduzierter Kettenlänge bestehen. Vor allem die Mechanismen des Laserabtrags sind aufgrund des großen Interesses der Mikroelektronikindustrie an den Eigenschaften als Photoresist intensiv untersucht [67, 65]. Da sich jedoch in Abhängigkeit von der Wellenlänge, Bestrahlungsintensität und chemischer Reinheit des Targetmaterials große Unterschiede ergeben, sind sie teilweise immer noch umstritten. Es hat sich gezeigt, dass sich die Depositionsprodukte durch die Variation der üblichen Parameter wie Laserenergie und Hintergrundgasdruck nur unzureichend variieren lassen, weshalb ein umfangreiches Verständnis der ablaufenden Prozesse unausweichlich für Verbesserungen ist.

Ziel dieser Arbeit ist es, diese Prozesse, die zur Ablation und zu den charakteristischen Eigenschaften der Filme führen, besser zu verstehen und aus diesem Verständnis Methoden zu entwickeln, diese Schichteigenschaften zu verbessern. Dabei wird besonderer Wert auf den Erhalt der chemischen Struktur des Polymers und die Oberflächenmorphologie der Filme gelegt. Weiterhin sollen die mechanischen Eigenschaften der Filme untersucht und die ihnen zugrundeliegenden Mechanismen aufgeklärt werden. 
Die Arbeit ist folgendermaßen gegliedert: In Kapitel 2 werden die experimentellen Details, die für diese Arbeit verwendet wurden, erläutert. Im Besonderen sollen die mechanischen Eigenschaften der deponierten Filme im Detail untersucht werden. Da diese sowohl von der Temperatur als auch von der betrachteten Messzeit abhängen, bietet sich die Methode der mechanischen Spektroskopie an. Zu diesem Zweck wurde eine neue spektroskopische Untersuchungsmethode, das Plasma Puls angeregte Reed (Plasma Plume eXcited Reed, PPXR) im Rahmen dieser Arbeit entwickelt [102], mit dem es möglich ist, in Kombination mit der PLD-Apparatur flexibel, in-situ und mit variabler Frequenz mechanische Spektroskopie zu betreiben. Dieser Aufbau wird in Kapitel 2 mit den anderen experimentellen Methoden kurz skizziert und in Kapitel 3 detailliert beschieben.

In Kapitel 4 werden die beobachteten Phänomene der Laserdeposition von PMMA unter üblichen Depositionsbedingungen gezeigt und mit den umfangreichen Ergebnissen aus der Literatur sowie zusätzlichen eigenen Arbeiten diskutiert.

Aufgrund der gewonnenen Erkenntnisse soll in Kapitel 5 untersucht werden, in wie fern durch eine Veränderung der Targetzusammensetzung die Deposition beeinflusst werden kann. Ein Ziel ist dabei, Schichten mit geringerer chemischer Veränderung im Vergleich zum Ausgangsmaterial zu erreichen und gleichzeitig eine verbesserte Kontrolle über die Morphologie der Filme zu erhalten. Dazu werden verschiedene Wege vorgestellt, angefangen von einfacher Beimengung anderer Materialien, über chemische Veränderungen bis hin zu einer in den letzten Jahren neu entwickelten Methode, der Matrix Assisted Pulsed Laser Evaporation (MAPLE) [87], die für diese Arbeit in Göttingen neu aufgebaut wurde.

Die Ergebnisse der mechanischen Spektroskopiemessungen werden in Kapitel 6 gezeigt und im Hinblick auf die besonderen Eigenschaften von laserdeponiertem PMMA diskutiert. Dabei wird auch die neue Methode PPXR mit konventionelleren Methoden verglichen und die Besonderheiten diskutiert. Im Weiteren wird diskutiert, in wie fern aus den Ergebnissen der mechanischen Spektroskopie auf den Mechanismus der gepulsten Laserdeposition zurückgeschlossen werden kann.

In Kapitel 7 werden die gewonnenen Ergebnisse dieser Arbeit kurz zusammengefasst. 


\section{Experimentelle Methoden}

\subsection{Gepulste Laserdeposition (PLD)}

Die gepulste Laserdeposition (Pulsed Laser Deposition, PLD) ist eine flexible Methode zur Herstellung dünner Filme verschiedener Materialien [18, 62, 29]. So lassen sich verschiedene Materialklassen wie Keramiken [131, 39], Halbleiter [136], Metalle [43] und, mit einigen Einschränkungen, auch die in dieser Arbeit untersuchten Polymere [8] deponieren (Eine ausführliche Zusammenfassung findet sich z. B. in [135]).

Für die Deposition wird ein Strahl eines gepulsten Lasers mit hoher Energie durch ein Fenster in eine Vakuumkammer geleitet und dort auf ein Target, welches aus dem zu deponierenden Material besteht, fokussiert. Dieses wird durch die Energie des Lasers abgetragen und das abgetragene Material schlägt sich auf dem einige Zentimeter entfernt gegenüberliegenden Substrat nieder und bildet dort einen Film. Für Polymere ist der Ablationsund Depositionsmechanismus kompliziert und immer noch Gegenstand von Diskussionen [19]. Er wird unter Berücksichtigung der im Rahmen dieser Arbeit gefundenen Ergebnisse ausführlich in Kapitel 4 diskutiert. Für Metalle ist er gut verstanden. Das Material wird zunächst thermisch verdampft und der Dampf oberhalb des Targets durch den Laser weiter erhitzt und ionisiert. Das Plasma breitet sich in einer Plasmakeule (engl.: plasma plume) mit hohen Geschwindigkeiten aus und die Ionen erreichen Energien von ca. 100 eV [34, 136]. Zusätzlich ergibt sich durch das gepulste Verfahren eine im Vergleich zu anderen Verfahren sehr hohe momentane Depositionsrate von typischerweise 0,1 Monolagen pro $\mu \mathrm{s}$.

Durch diese Besonderheiten zeichnen sich laserdeponierte Filme durch besondere Eigenschaften aus. Sie verfügen i. A. über eine hervorragende Substrathaftung und eine gut einstellbare stöchiometrische Zusammensetzung [60]. Eine besondere Eigenschaft sind je nach Material verschieden ausgeprägte, tröpfchenförmige Erhöhungen, so genannte Droplets, die auf Prozesse am Target zurückzuführen sind [33]. Die Dichte der Filme ist hoch und sie stehen i. A. unter hohen Druckspannungen [99, 100]. Oberfläche und Grenzflächen sind bei geeigneten Depositionsbedingungen glatt mit rms-Rauigkeiten unter einem Nanometer, und die Filmdicken sind sehr gut kontrollierbar [131, 39], jedoch nur mit erheblichem Aufwand auf einen Bereich größer als $2 \times 2 \mathrm{~cm}^{2}$ homogen einstellbar. 


\subsubsection{Verwendeter Aufbau}

Eine der beiden verwendeten PLD-Apparaturen ist in Abbildung 2.1 gezeigt, um das Prinzip zu verdeutlichen. Von der linken Seite kommt der Laser durch ein UV-durchlässiges

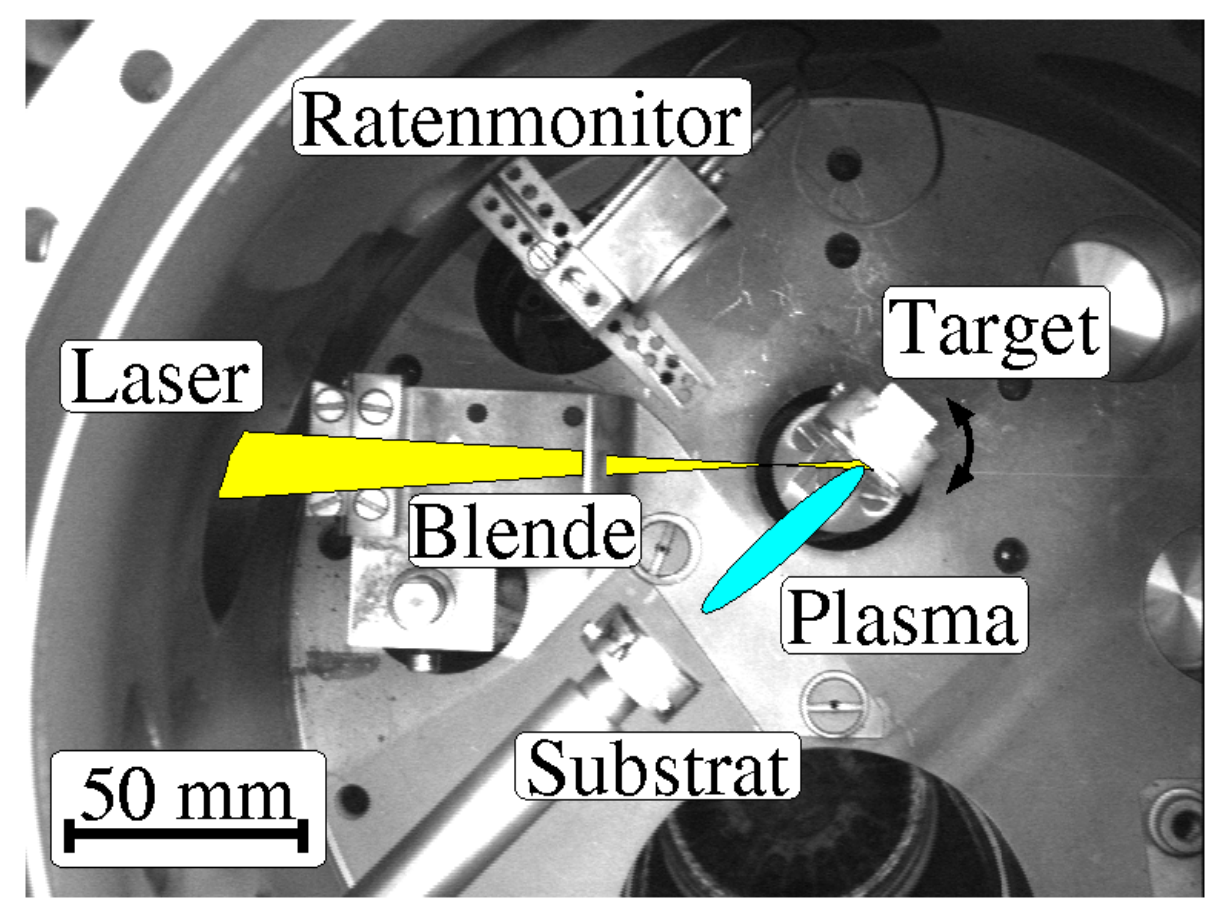

Abbildung 2.1: Schematischer Aufbau der PLD-Apparatur

Fenster. Verwendet wurde ein KrF Excimer-Laser (LAmbDa Physik LPX 110i) mit $248 \mathrm{~nm}$ Wellenlänge und einer Pulsdauer (FWHM) von 30 ns. Die Repetitionsfrequenz wurde für die Polymere auf $5 \mathrm{~Hz}$ reduziert. Der Einfallswinkel des Laserstrahls auf dem Target beträgt $45^{\circ}$. Für die Silber-Targets wurde elementares Silber mit einer Reinheit von mindestens 99,99\% verwendet. Die Herstellung der Polymertargets wird in Abschnitt 2.1.2 beschrieben.

Im Weiteren wurden zwei leicht verschiedene Depositionsgeometrien verwendet. Zum einen die fokussierende Geometrie, bei der der Fokus des Lasers auf der Probe liegt, zum anderen die abbildende Lasergeometrie, wobei das Laseraustrittsfenster optisch auf die Probe abgebildet wird. Letztere zeichnet sich gegenüber der fokussierenden Geometrie durch eine deutlich höhere Energiehomogenität des Laserstrahls auf dem Target aus [11. Jedoch ist in keiner der hier untersuchten Größen zwischen diesen beiden Geometrien ein signifikanter Unterschied festgestellt worden, weshalb sie für diese Arbeit als gleichwertig erachtet werden. 
Die Brennfleckgröße des Lasers auf dem Target beträgt $2 \mathrm{~mm}^{2}$ und die Laserenergie für die Polymere, sofern nicht anders angegeben, 60 mJ/Puls. Für Silber wurde eine deutlich höhere Energie verwendet, üblicherweise etwa 130 mJ. Der Target-Substrat-Abstand beträgt $40 \mathrm{~mm}$ in der fokussierenden und $54 \mathrm{~mm}$ in der abbildenden Geometrie. Auch für diese Unterschiede konnte außer einer veränderten Depositionsrate (siehe [115]) kein Unterschied festgestellt werden.

Der Druck in der Depositionskammer ist vor der Deposition niedriger als $5 \cdot 10^{-8}$ mbar, für die MAPLE-Experimente (Abschnitt 2.1.3 $1 \cdot 10^{-6}$ mbar. Während der Laserdeposition von Polymerschichten steigt der Druck bis in den Bereich $5 \cdot 10^{-4}$ mbar an, fällt nach der Beendigung der Deposition jedoch sehr schnell wieder in den ursprünglichen Bereich ab.

In die UHV-Kammer lassen sich mehrere Targets gleichzeitig einbauen und werden über Schrittmotoren automatisiert in den Strahlengang gefahren. Weiterhin werden die Targets während der Deposition relativ zum Laserstrahl bewegt, um eine größere Fläche abzutragen und die Targets gleichmäßiger altern zu lassen. Zusätzlich können sowohl Targets als auch Substrate in die Kammer eingeschleust werden, was ein Brechen des Vakuums erspart.

Bei der Deposition auf geheizten Substraten wurden diese auf einen PID-geregelten Widerstandsofen mit einem Target-Substrat-Abstand von $40 \mathrm{~mm}$ geklemmt. Die Temperaturmessung erfolgt hierbei über ein Thermoelement, dass seitlich mit Leitsilber an das Substrat geklebt wird. Diese Experimente wurden ausschließlich in der fokussierenden Geometrie durchgeführt, um eine maximale Reproduzierbarkeit zu erreichen.

Als Substrate wurden polierte (111)-Siliziumeinkristalle verwendet, für die Infrarotspektroskopie (Abschnitt 2.2.4) wurden wegen der besseren Reflektivität Al-Substrate benutzt. Um Schichtdickenoszillationen im IR-Spektrum zu unterdrücken, wurde hierfür unpoliertes, technisches Aluminium verwendet.

Für die Messung der Depositionsrate wird in der abbildenden Geometrie in-situ ein Ratenmonitor (LEyBOLd InfICON XTM/2 Deposition Monitor) mit einer Auflösung von $1,2 \mathrm{ng} / \mathrm{cm}^{2}$ verwendet, der sich in einem Abstand von $54 \mathrm{~mm}$ direkt gegenüber des Targets befindet.

\subsubsection{Polymertargets}

Für die Herstellung der Polymertargets wurde PMMA (ALDRICH) mit einer Molmasse von $996 \mathrm{~kg} / \mathrm{mol}$ verwendet. Dieses wurde als Pulver in einer Presse bei $3 \cdot 10^{7} \mathrm{~N} / \mathrm{cm}^{2}$ für etwa eine Stunde über die Glastemperatur erhitzt. Auf diese Weise erhält man klare, feste Targets. 
Für die Beimengung von Antracen (Abschnitt 5.1) wurden 3 g PMMA-Pulver mit der entsprechenden Menge an Antracen eingewogen, vermischt und in der Kugelmühle mit 10 Stahlkugeln mit 10 mm Durchmesser eine Stunde fein vermahlen. Die daraus gepressten Targets sind optisch homogen und zeigen eine je nach Antracenanteil deutlicher ausgeprägte weißliche bis hellgelbe Farbe. Mit 3 Gew.\% Antracen sind die Targets dabei transparent, mit $10 \%$ nicht.

\section{Kontrollierte radikalische Polymerisation}

Targets mit Ausgangsmolmassen von $3200 \mathrm{~g} / \mathrm{mol}$ wurden in Zusammenarbeit mit dem Institut für Physikalische Chemie, Universität Göttingen, hergestellt. Für die kontrollierte radikalische Polymerisation wurde das Monomer MMA in Toluol gelöst und mit einer hohen Konzentration $\left(\sim 0,3\right.$ Gew.\%) des Starters AIBN $\left(\mathrm{NCCH}_{2} \mathrm{~N}=\mathrm{NCH}_{2} \mathrm{CN}\right)$ versetzt. Dieser bildet oberhalb von $60{ }^{\circ} \mathrm{C}$ Starterradikale. Die komplette Polymerisationsreaktion ist in Abbildung 2.2 dargestellt. Die Terminierung wurde durch die Zugabe von 2,9 Vol.\% DDM

1. Starterreaktion
\[ \mathrm{NC}-\mathrm{CH}_{2}-\mathrm{N}=\mathrm{N}-\mathrm{CH}-\mathrm{CN} \stackrel{\mathrm{T}}{\longrightarrow} 2 \mathrm{NC}-\mathrm{CH}_{2} \bullet+\mathrm{N}_{2} \]

2. Initierung<smiles>C=C(C)C(=O)OCCNCC#N</smiles>

3. Propagation

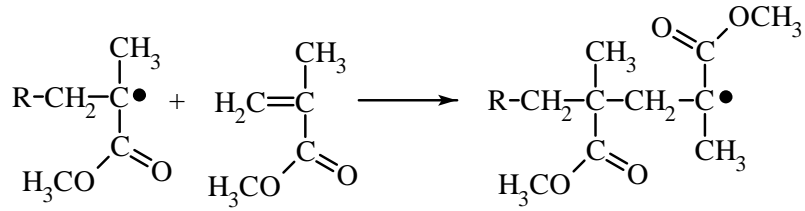

4. Terminierung

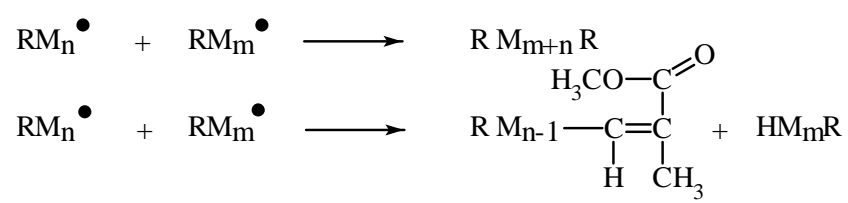

Abbildung 2.2: Radikalische Polymerisation von PMMA 
(Dodecaethiol, $\mathrm{CH}_{3}\left(\mathrm{CH}_{2}\right)_{11} \mathrm{SH}$ ) als Regler (chain transfer agent) beschleunigt, um kurze Kettenlängen zu realisieren. Der Prozess der Terminierung durch DDM ist in Abbildung 2.3 gezeigt. Nach der Polymerisation wurde das Toluol im Vakuum verdampft und es ergibt

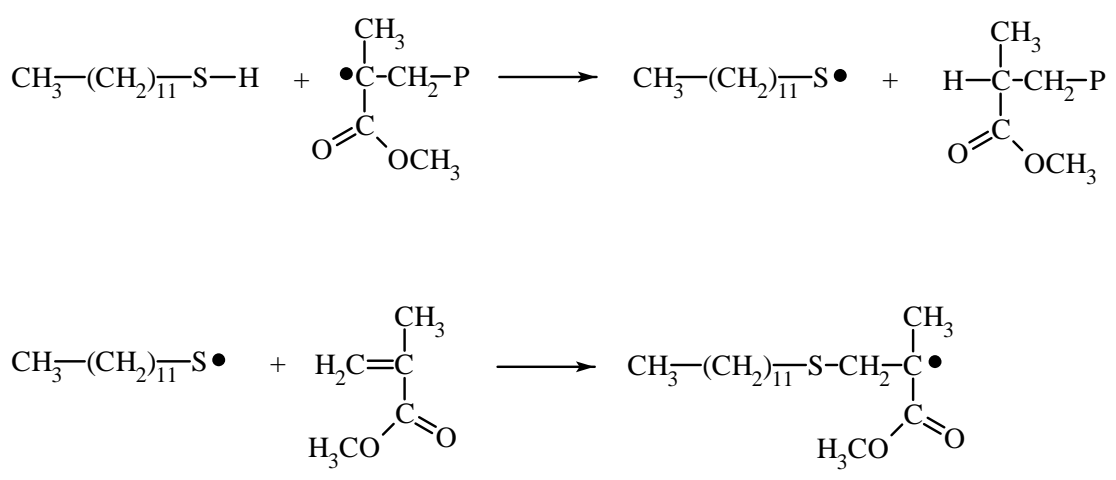

Abbildung 2.3: Terminierung der Polymerisation durch DDM

sich ein schaumartiges Material, das zu Pulver zerstampft und danach mit dem schon beschriebenen Verfahren in Targetform gepresst wurde.

\section{Reversible-Addition-Fragmentation chain Transfer polymerization (RAFT)}

Die Reversible-Addition-Fragmentation chain Transfer polymerization (RAFT) gehört zu den lebenden Polymerisationen [80]. Das RAFT-Reagenz, in unserem Fall 2-Cyanoprop-2yldithiobenzoat, bildet mit einem Polymerradikal eine intermediäre Verbindung und stabilisiert es so. Nach dem Schema in Abbildung 2.4 wird das aktive Ende an die nächste Polymerkette transferiert. Auf diese Weise erhält man eine gute Kontrolle über das nun

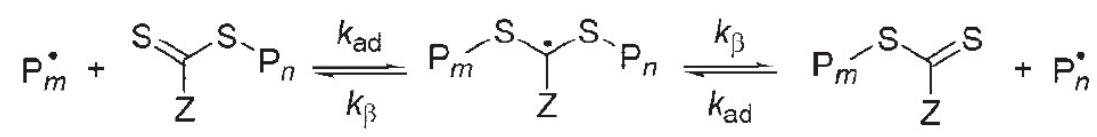

Abbildung 2.4: Schema der RAFT-Polymerisation[12], Z ist in diesem Fall ein einfacher aromatischer Ring.

mit dem Umsatz lineare Wachstum der Ketten. Die Fähigkeit zur Polymerisation wird nicht durch statistische Terminierung beendet, sondern nur durch Deaktivierung mittels Terminatoren. Dadurch ergibt sich die Möglichkeit, scharfe Kettenlängenverteilungen zu erhalten, sowie durch aufeinander folgende Zugabe verschiedener Monomere in einem weiten Maß sehr definierte Copolymere zu synthetisieren. 
Auf diese Weise wurde in Zusammenarbeit mit dem Institut für Physikalische Chemie, Universität Göttingen, PMMA mit einer mitteleren Molmasse von $M_{W}=16 \mathrm{~kg} / \mathrm{mol}$ und ein ebenso schweres Copolymer aus MMA und Styrol synthetisiert. Das Polymerpulver wurde in der oben beschriebenen Weise gepresst und ergibt feste Targets, die durch die Chromophoren des RAFT-Reagenz eine hellrote Farbe zeigen.

\subsubsection{MAPLE}

Eine der neuesten Entwicklungen der gepulsten Laserdeposition stellt die so genannte $M a$ trix Assisted Pulsed Laser Evaporation (MAPLE) dar [87, 138. Hierfür wird das Material, das deponiert werden soll, zuerst in einem geeigneten Lösungsmittel gelöst. Dieses hat während der Ablation die Aufgabe, die Laserenergie zu absorbieren und in Wärme umzuwandeln. Auf diese Weise wird das eigentliche Targetmaterial deutlich weniger der schädigenden Wirkung der UV-Strahlung des Lasers ausgesetzt. Damit ergibt sich als Anforderung für das Lösungsmittel, dass dieses möglichst stärker die UV-Strahlung absorbiert als das eigentliche Targetmaterial. Um ein festes Target zu erhalten, das auch im UHV verwendbar ist, wird die Lösung in flüssigem Stickstoff gefroren.

Für dieses Verfahren wurde im Rahmen dieser Arbeit ein spezieller Targethalter konstruiert. Ein im UHV befindlicher Kupferblock wird über doppelwandige Rohrzuführungen im Durchfluss mit flüssigem Stickstoff gekühlt, um das Target über längere Zeit im festen Aggregatszustand und den Dampfdruck im UHV-kompatiblen Bereich zu halten.

Der eigentliche Targethalter besteht aus einem weiteren $\mathrm{Cu}$-Block, in den eine Versenkung gefräst wurde. In diese wird das flüssige Targetmaterial eingefüllt und dann der Block in flüssigem Stickstoff schockgefroren. In diesem Stadium verhindert der über dem Bad liegende, gasförmige Stickstoff weitgehend die Kondensation von Wasser auf dem Target. Eine Skizze des verwendeten Aufbaus ist in Abbildung 2.5 links gezeigt. Im rechten Teil der Abbildung ist ein MAPLE-Target in dem Cu-Block nach dem Laserbeschuss zu sehen. Das feste Target wird auf den im UHV abgekühlten Targetkühler aufgeschraubt. Die Kammer wird hierfür mit Ar belüftet. Da sie oben geöffnet wird und Ar etwas schwerer als Luft ist, kann kein Wasser kondensieren. Das am Kühlhalter kondensierte Ar wird unter dem Halter aufgefangen und verdampft beim darauf folgenden Abpumpen im Vakuum sehr schnell. Der Transfer des Targets muss allerdings schnell erfolgen, damit dieses nicht aufschmilzt oder von Eis bedeckt wird. Daraufhin wird die Kammer wieder auf einen Hintergrundgasdruck unter $1 \cdot 10^{-6}$ mbar abgepumpt. Dieses Verfahren kann nur als De- 


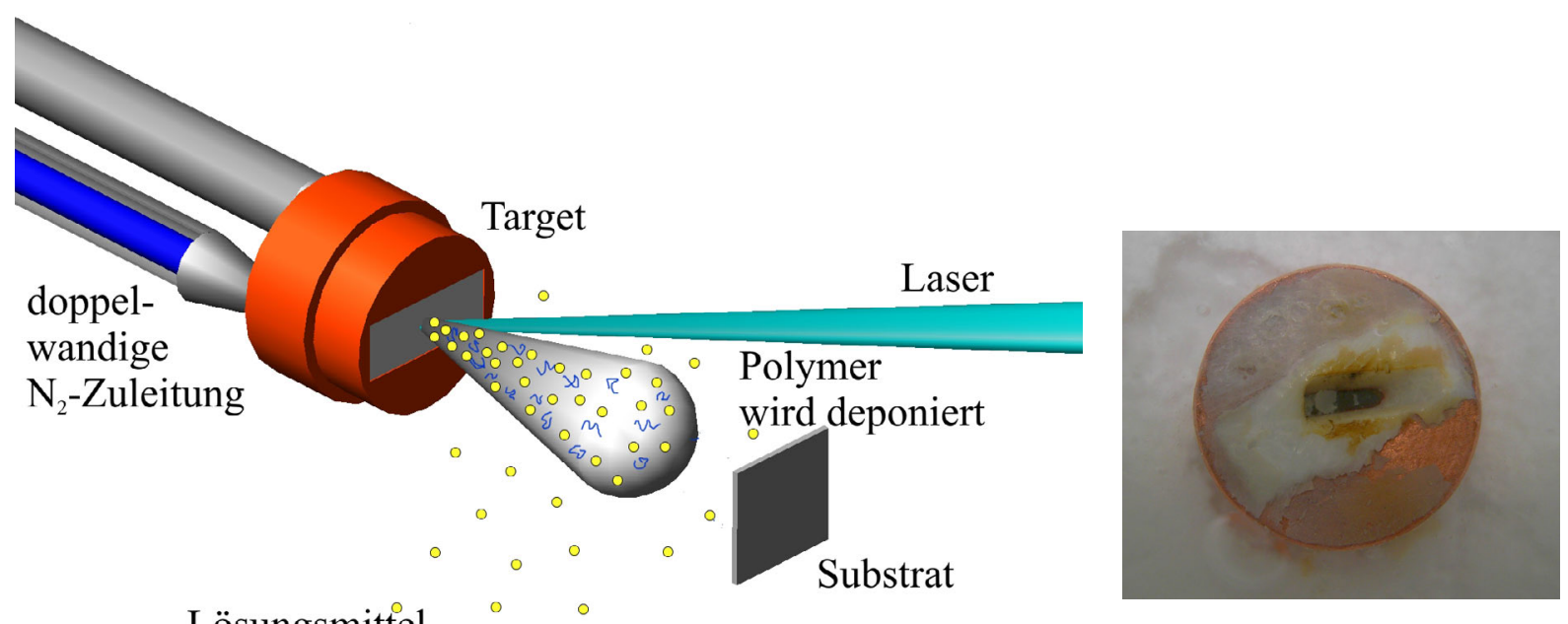

Lösungsmittel 。 wird abgepumpt

Abbildung 2.5: Links: Schematische Skizze des MAPLE-Aufbaus; Rechts: Mit 5.000 Laserpulsen bis auf den Halter durchgeschossenes Target; PMMA in Toluol, gefroren in flüssigem Stickstoff

monstrationsaufbau betrachtet werden, jedoch verwenden andere Gruppen als Unterschied höchstens einen beheiz- und bewegbaren Targethalter [138].

Als Lösungsmittel wurden Dimethoxy-Ethan (DME) und Toluol verwendet, da sie bei 248 nm gut absorbieren und PMMA sehr gut lösen. Sie besitzen einen Schmelzpunkt von $-58^{\circ} \mathrm{C}$, respektive $-95^{\circ} \mathrm{C}$, sind also in flüssigem Stickstoff gut handhabbar. Allerdings ist es gerade für Toluol nicht mehr möglich mit gasförmigem Stickstoff zu kühlen, wie dies von anderen Gruppen für andere Lösungsmittel beschrieben wird [94, 125]. Die Lösungen wurden jeweils mit 0,1-2 Gew.\% PMMA angesetzt und durch Schütteln und Wartezeit von mehreren Stunden gelöst. Ultraschall wurde in diesem Fall vermieden, da dieses unter Umständen schon die Proben für die Chromatographie zerstören könnte.

Für die MAPLE-Deposition wurde die Linse aus der Fokussierungsebene bewegt, um eine größere bestrahlte Fläche und geringere Energiedichte zu erhalten. Die mittlere Energiedichte lag damit bei etwa $170 \mathrm{~mJ} / \mathrm{cm}^{2}$. 


\subsection{Charakterisierungsmethoden}

\subsubsection{Plasma Puls angeregtes Reed (PPXR)}

Für die mechanische Spektroskopie an laserdeponierten Filmen wurde hier eine neue Methode, das Plasma Puls angeregte Reed (Plasma Plume eXcited Reed, PPXR), entwickelt 102. Die Apparatur besteht aus einem frei schwingenden Balken nach der Art eines vibrating reed [17], auf den die Schicht aufgebracht wird. Die Anregung erfolgt direkt über die Energie des Laser-Plasmas mit einem Silbertarget. Die Schwingung dieses Balkens wird durch einen Lichtzeiger gemessen und Schwingungsfrequenz und Dämpfung ausgewertet. Da die Messung temperaturabhängig erfolgen muss, ist der Balken geregelt heizbar. Das Prinzip ist in Abbildung 2.6 skizziert, eine detaillierte Beschreibung findet in Kapitel 3
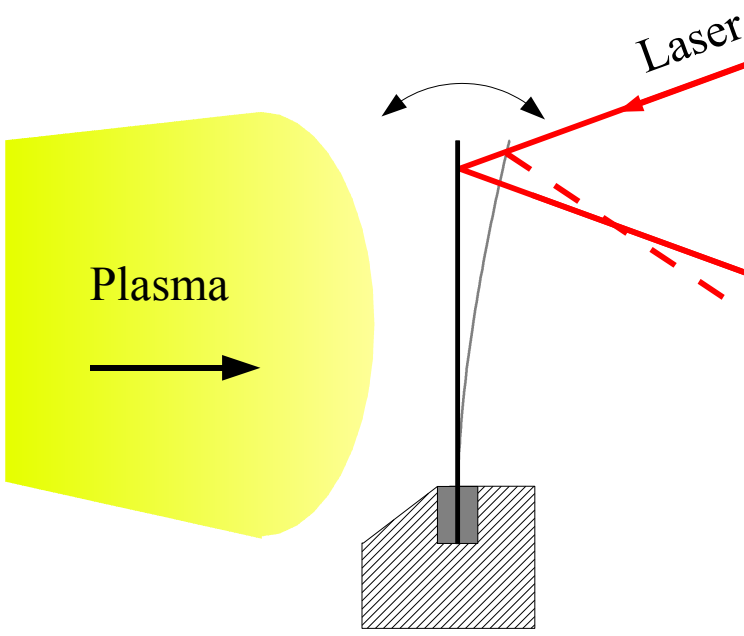

Abbildung 2.6: Skizze des Plasma Puls angeregten Reed, PPXR

statt.

\subsubsection{Doppelpaddeloszillator (DPO)}

Als ex-situ Methode für die mechanische Spektroskopie wurde in Zusammenarbeit mit dem 1. Physikalischen Institut der Universität Göttingen der Doppelpaddeloszillator verwendet. Hierbei handelt es sich um eine Substratgeometrie, die darauf optimiert wurde, bei Schwingung in einer antisymmetrischen Torsionsmode möglichst wenig Bewegung in der Einspannung zu erzeugen. Bei einer Resonanzfrequenz von ca. $5,4 \mathrm{kHz}$ erfolgt damit 
eine sehr geringe Energiedissipation und die Grunddämpfung ist sehr gering. Eine genaue Beschreibung der Apparatur findet sich bei Rösner [97, 95].

\subsubsection{Dielektrische Spektroskopie (DS)}

Als alternative Spektroskopiemethode für die laserdeponierten Polymerfilme wurde die dielektrische Verlustspektroskopie [26] in Zusammenarbeit mit dem 1. Physikalischen Institut, Universität Göttingen, und dem Lehrstuhl für Experimentalphysik V, Universität Augsburg, durchgeführt. Dazu wurden die Proben auf Cu-Substrate deponiert und als zweite Elektrode für die kapazitive Messung ein Cu-Film deponiert. Die Messung erfolgte an einem Novocontrol $\alpha$-Analyzer.

\subsubsection{Fourier Transformations IR-Spektroskopie (FTIR)}

Zur Untersuchung der chemischen Bindungsverhältnisse werden Infrarotspektren mittels Fourier Transformations Infrarot Spektroskopie (FTIR) aufgenommen. Die Spektren wurden an auf rauem Aluminium hergestellten Filmen in Reflektion gemessen. Dabei durchläuft das infrarote Licht die Schicht, wird am Substrat reflektiert und durchläuft sie ein zweites Mal, womit die Transmission der Schicht gemessen wird. Verwendet wurde ein Bruker Tensor 37 mit stickstoffgekühltem MCT-Detector (HgCdTe) und die computergesteuerte Winkelverfahreinheit HARRICK Auto Seagul bei einem Einfallswinkel (zur Oberfläche) von $54^{\circ}$. Es wurde eine Auflösung von $4 \mathrm{~cm}^{-1}$ gewählt und das Spektrum um vorher aufgenommene Hintergrundspektren korrigiert. Der Spektrometerraum wurde mit getrockneter Luft gespült, um Wasserbanden effektiv zu reduzieren.

\subsubsection{Größenausschlusschromatographie (SEC)}

In Zusammenarbeit mit dem Institut für Physikalische Chemie, Universität Göttingen, wurden mithilfe der Größenausschlusschromatographie (Size Exclusion Chromatographie, SEC) die molekularen Massen der Polymere bestimmt. Eine Lösung des zu untersuchenden Materials wird hierzu durch ein poröses Trenngel geleitet. Da die kleinen Moleküle auf dem Weg durch die Säulen mehr Volumen zur Verfügung haben als die großen, brauchen sie für den Durchlauf länger. Auf diese Weise erfolgt eine im Idealfall rein physikalische Trennung der verschiedenen Molekülgrößen, d.h. keine chemische Wechselwirkung mit dem Trenngel. Die unterschiedlichen Ankunftszeiten werden mit Standards auf die Molekülgewichte kalibriert. 
Verwendet wurde ein WATERS System mit drei PSS-SDV Säulen mit einer Partikelgröße von $5 \mu \mathrm{m}$ und einem Porendurchmesser von $10^{4}, 10^{2}$ und $10 \mathrm{~nm}$. Als Eluent wurde Tetrahydrofuran (THF), bei einer Durchflussmenge von $1 \mathrm{ml} / \mathrm{min}$ benutzt. Zur Kalibrierung wurden vier verschiedene PMMA-Eichstandards (POLYMER Standards SERvicE, PSS, $800 \mathrm{~g} / \mathrm{mol}<M_{P}<1,8 \cdot 10^{6} \mathrm{~g} / \mathrm{mol}$ ) verwendet.

Die deponierten PMMA-Schichten wurden mit THF vom Substrat gelöst. Nach ca. 24 Stunden ist dieses Lösungsmittel bei Raumtemperatur verdampft, die Proben werden weiter bei Unterdruck im Exsikkator getrocknet und nach weiteren 24 Stunden gewogen. Vor der SEC Untersuchung werden sie wieder für mindestens eine Stunde in THF gelöst.

\subsubsection{Rasterelektronenmikroskopie (SEM)}

Für die Rasterelektronenmikroskopischen Aufnahmen wurden drei verschiedene Mikroskope verwendet, ein CAmbridge Instruments S360 (W-Kathode, SE-Detektor), ein PHILIPS SEM 515 ( $\mathrm{LaB}_{6}$ Kathode, SE-Detektor) und ein LEO Supra 35 (Feldemmissionskathode, SE+Inlens Detektor). Um das Polymer möglichst wenig zu beschädigen, wurden Beschleunigungsspannungen von nur 5-10 kV benutzt. Gegen die elektrostatische Aufladung wurden die Proben vor dem Einschleusen ins Rasterelektronenmikroskop mit einer 20$30 \mathrm{~nm}$ dicken Goldschicht bedampft.

\subsubsection{Rasterkraftmikroskopie (AFM)}

Mit Hilfe der Rasterkraftmikroskopie (Atomic Force Microscopy, AFM) lassen sich Höheninformationen über die Probe mit hoher Präzision und Auflösung gewinnen. Verwendet wurde ein VEeco NanoScope IV Multimode AFM im TappingMode. Die Bildauswertung erfolgte über das Programm NanoScope Version 6.13R1 der Firma VEECo.

\subsubsection{Röntgenreflektometrie (XRR)}

Bei Filmen mit geringer Schichtdicke und Rauigkeit, lassen sich diese mittels Röntgenreflektometrie (X-Ray Reflectometry XRR), also spekulärer Kleinwinkelröntgenstreuung, untersuchen. Hierfür wurde ein PHILIPS X'Pert Vierkreis-Röntgendiffraktometer mit Co$K_{\alpha}$-Röntgenröhre $(0,17902 \mathrm{~nm})$ und Monochromator verwendet. Die erhaltenen Reflektogramme wurden mithilfe des Softwarepakets IMD [137] angepasst, bei dem der rekursive Parrat Algorithmus [85] verwendet wird. 


\section{Entwicklung des Plasma Puls angeregten Reed (PPXR)}

Als Ergänzung für die vorhandenen Untersuchungsmethoden wurde für die mechanische Spektroskopie an laserdeponierten Filmen im Rahmen dieser Arbeit, das Plasma Puls angeregte Reed (Plasma Plume eXcited Reed, PPXR) entwickelt und aufgebaut [102]. Das Grundprinzip der Anlage entspricht dem eines konventionellen vibrating reed [17], allerdings sind die Dimensionen den Schichten der gepulsten Laserdeposition angepasst und die Anregung des Schwingungsbalkens erfolgt über die Plasmakeule (engl.: plasma plume) der gepulsten Laserdeposition (PLD).

Im Folgenden soll nun zuerst die Grundidee hinter dem Prinzip des PPXR eingeführt werden. Ausgehend von den Schwingungsmoden des vibrating reed soll anhand eines einfachen Modells die Anregung des PPXR durch die Plasmakeule untersucht werden. Im Weiteren wird der experimentelle Aufbau der PPXR Apparatur sowie der Versuchsablauf und die speziell entwickelte Auswertung beschrieben. Zum Schluss wird erläutert, wie aus den mittels PPXR gemessenen Dämpfungskurven und Resonanzfrequenzen der komplexe elastische Modul bestimmt werden kann.

\section{$3.1 \quad$ Idee}

Für die mechanische Spektroskopie benötigt man eine periodische Bewegung des zu messenden Systems. Üblicherweise benutzt man resonante Methoden, lässt also die Probe in einer Eigenschwingung des messenden Systems schwingen. Aus je zwei der (temperaturabhängigen) Parameter Dämpfung, Veränderung der Resonanzfrequenz und Phasenlage zwischen Anregung und Schwingung lässt sich der komplexe elastische Modul bestimmen.

Ein etabliertes resonantes Verfahren ist das so genannte vibrating reed. Hier wird ein länglicher Balken mit meist rechteckigem Querschnitt an einer Seite fest eingespannt und kann nach der Anregung an der anderen frei schwingen. Der Hauptvorteil des vibrating reed für die mechanische Spektroskopie ist der einfache experimentelle Aufbau bei recht geringer Grunddämpfung. Für die Untersuchung dünner Filme wählt man i. A. einen Balken aus Silizium, da dies eine geringe Grunddämpfung hat, so dass der Film darauf einen 
möglichst großen Anteil an der Gesamtdämpfung hat. Dadurch ist es einfach möglich, die Energiedissipation im leeren Substrat und der Aufhängung, die durch eine Leermessung bestimmt wird, von der des Gesamtsystems abzuziehen und damit auf die Eigenschaften des Films zu schließen. Die Auswertung einer solchen Messung wird im Detail in Abschnitt 3.7 beschrieben.

Problematisch ist für diese Methode die Anregung der Schwingung. Mechanische Anregung, wie sie für bulk-Proben üblich ist (z. B. [52]), fällt wegen der Zerbrechlichkeit der dünnen Siliziumsubstrate aus. Bei der ebenfalls typischen kapazitiven Anregung [48] ist bei in-situ Verwendung mit einem Depositionsverfahren zu beachten, dass die Elektroden beschichtet werden. Um diese Nachteile zu umgehen wird bei dem in dieser Arbeit neu entwickelten PPXR-Verfahren die Energie der Plasmakeule der gepulsten Laserdeposition benutzt. Der ruhende Balken wird mit einem einzelnen Plasmapuls aus der Gleichgewichtslage gebracht, damit zur Schwingung angeregt und kann dann, nur durch die Dämpfung bestimmt, ausklingen (free decay). Zusätzlich ergibt sich der Vorteil, dass das Substrat automatisch in der ersten Eigenmode schwingt, was die sonst recht aufwendige Suche nach der scharfen Resonanzfrequenz erspart.

Für die Anregung reicht ein einziger Puls aus, so dass, wenn zur Anregung beispielsweise ein Ag-Target verwendet wird, pro Messpunkt nur etwa 0,1 Monolage Ag deponiert wird. Für zu messende Filmdicken von mehr als $100 \mathrm{~nm}$ sollte der dadurch auftretende Fehler auch bei den hier jeweils verwendeten 300-400 Messpunkten zu vernachlässigen sein [102.

\subsection{Beschreibung der Schwingung}

\subsubsection{Balkenbiegung}

Für die mathematische Beschreibung der Schwingung (siehe [84, 38]) wird das links in Abbildung 3.1 skizzierte Koordinatensystem verwendet. Auf der rechten Seite der Abbildung ist ein durch die Verbiegung verformtes Volumenelement skizziert. Die lange Kante des Balkens zeigt in $x$-Richtung und die Verbiegung erfolgt in $z$-Richtung. Der Ursprung liegt in $y$ und $z$ in der Mitte der Einspannstelle. Die Dicke des Balkens sei $d$, die Breite $b$ und die Länge $l$.

Die orts- und zeitabhängige Mittellinie des Balkens bezeichnen wir mit $u(x, t)$. Zur Ver-

einfachung gehen wir von kleinen Ausschlägen aus, d. h. $\left|u^{\prime}\right| \ll 1$. Damit ist die Änderung der Ausdehnung in $x$-Richtung zu vernachlässigen. 

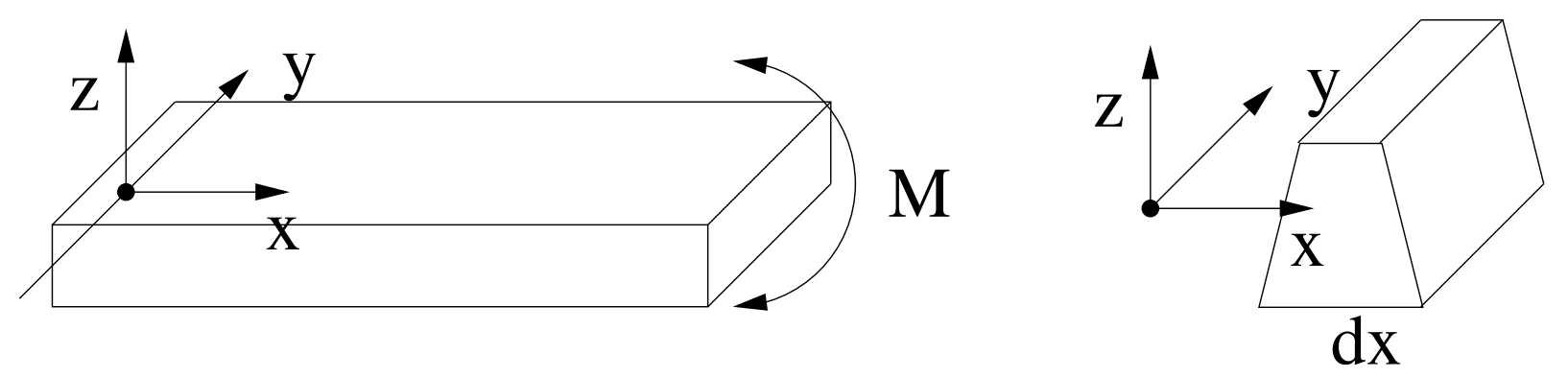

Abbildung 3.1: Koordinatensystem für die Beschreibung der Schwingung

Die kinetische Energie $E_{\text {kin }}$ des schwingenden Balkens ergibt sich zu

$$
E_{\text {kin }}=\frac{\rho}{2} \int_{0}^{l} d x\left(\frac{\partial u(x, t)}{\partial t}\right)^{2}=\frac{\rho}{2} \int_{0}^{l} d x \dot{u}^{2},
$$

wobei zur besseren Übersichtlichkeit $\rho$ die Masse pro Länge darstellt. Für kleine Auslenkungen gilt nun $u^{\prime \prime}(x, t) \approx 1 / R$ mit dem Krümmungsradius $R$. Damit lässt sich die potentielle Energie des ausgelenkten Balkens schreiben durch

$$
E_{\mathrm{pot}}=\frac{k}{2} \int_{0}^{l} d x\left(\frac{\partial^{2} u(x, t)}{\partial x^{2}}\right)^{2}=\frac{k}{2} \int_{0}^{l} d x u^{\prime \prime 2}
$$

Dabei ist $k=b \int_{-d / 2}^{d / 2} d z z^{2} E$ mit dem Elastizitätsmodul $\left(\sigma_{11}\right) E$ die Biegesteifigkeit (die gespeicherte Energie pro Länge und Krümmung), die die Dicke und Materialeigenschaften des Balkens mit einschließt. Für einen unbeschichteten Balken liegt die neutrale Faser in der Mitte, die Gleichung ist in dem oben gewählten Koordinatensystem leicht zu integrieren und ergibt

$$
k=b d^{3} E / 12 .
$$

Weiterhin wollen wir eine äußere Kraft $f(x, t)$ zulassen, die auf den Balken wirkt. Damit ergibt sich folgender Beitrag zur Energie:

$$
E_{\mathrm{f}}=-\int_{0}^{l} d x u(x, t) f(x, t)
$$

Aus diesen drei Beiträgen lässt sich die Lagrange-Funktion konstruieren:

$$
L=E_{\mathrm{kin}}-E_{\mathrm{pot}}-E_{\mathrm{f}}
$$


Daraus ergibt sich eine Lagrange-Dichte

$$
\mathfrak{L}\left(\dot{u}, u, u^{\prime \prime}\right)=\frac{\rho}{2} \dot{u}^{2}-\frac{k}{2} u^{\prime \prime 2}+u f(x, t) .
$$

Nun setzen wir $\mathfrak{L}\left(\dot{u}, u, u^{\prime \prime}\right)$ in die Euler-Lagrange-Gleichung

$$
\frac{d}{d t} \frac{\partial \mathfrak{L}}{\partial \dot{u}}-\frac{\partial^{2}}{\partial x^{2}} \frac{\partial \mathfrak{L}}{\partial u^{\prime \prime}}=\frac{\partial \mathfrak{L}}{\partial u}
$$

ein und erhalten

$$
\rho \ddot{u}+k u^{\prime \prime \prime \prime}=f(x, t)
$$

\section{Schwingung}

Für unseren Fall sind für $f(x, t)$ zwei Fälle wichtig. Zum einen ist das die von außen kräftefreie Schwingung, die nur durch die Dämpfung beeinflusst wird. Für diese lässt sich die Kraft mit $f(x, t)=-2 \lambda \dot{u}$ annehmen. Damit erhalten wir

$$
\rho \ddot{u}+k u^{\prime \prime \prime \prime}=-2 \lambda \dot{u} .
$$

Für diese lineare partielle Differentialgleichung hilft der übliche Separationsansatz $u(x, t)=$ $g(t) h(x)$. Damit erhalten wir

$$
\rho h(x) \ddot{g}(t)+2 \lambda h(x) \dot{g}(t)+k g(t) h^{\prime \prime \prime \prime}(x)=0
$$

oder auch

$$
-\frac{\ddot{g}(t)}{g(t)}-\frac{2 \lambda}{\rho} \frac{\dot{g}(t)}{g(t)}=\frac{k}{\rho} \frac{h^{\prime \prime \prime \prime}(x)}{h(x)} .
$$

Dies lässt sich in Eigenfunktion mit zugehörigen Eigenwerten auflösen, wenn man beide Seiten gleich einer Konstanten setzt, die wir mit $\omega_{0}^{2}$ bezeichnen wollen. Damit erhalten wir zwei gewöhnliche Differentialgleichungen:

$$
\begin{aligned}
& 0=\ddot{g}(t)+\frac{2 \lambda}{\rho} \dot{g}(t)+\omega_{0}^{2} g(t) \\
& 0=\frac{k}{\rho} h^{\prime \prime \prime \prime}(x)-\omega_{0}^{2} h(x) .
\end{aligned}
$$


Die Lösung für die zeitliche Entwicklung ergibt sich mit $\Lambda=\lambda / \rho$ und $\omega=\sqrt{\omega_{0}^{2}-\Lambda^{2}}$ zu

$$
g(t)=A_{0} \mathrm{e}^{-\Lambda t} \cos \omega t
$$

Für die Lösung von $h(x)$ definieren wir

$$
\gamma=\sqrt[4]{\rho \omega_{0}^{2} / k}
$$

und erhalten

$$
h(x)=B \sin (\gamma x)+C \cos (\gamma x)+D \sinh (\gamma x)+E \cosh (\gamma x)
$$

Wir haben drei Randbedingungen, $u(0)=u^{\prime}(0)=u^{\prime \prime}(l)=0$. Damit bleibt ein freier Parameter übrig, die Gesamtamplitude. Im Übrigen erhalten wir nichttriviale Lösungen für die Lösungen der Determinantengleichung

$$
1+\cos (\gamma l) \cosh (\gamma l)=0
$$

die numerisch ermittelt werden können. Die erste Lösung liegt bei $\gamma l=1,8751$. Damit erhalten wir auch die Kreisfrequenz $\omega_{0}$ der Schwingung, aus Gleichung 3.4 folgt

$$
\omega_{0}=\sqrt{\frac{k}{\rho}} \gamma^{2}
$$

Die übrigen Parameter bestimmen sich zu

$$
C=-B \frac{2 \sin \gamma l+\sinh \gamma l}{2 \cos \gamma l+\cosh \gamma l}, \quad D=-B \quad \text { und } \quad E=-B C
$$

\section{Auslenkung durch die Anregung}

Die andere interessante äußere Kraft ist die Anregung des Balkens durch die ankommenden Ionen der Plasmakeule. Diese Kraft ist über dem Ort annähernd konstant, zeitlich allerdings nicht. In diesem Fall ist die auftretende partielle Differentialgleichung nach Gleichung 3.3 nicht mehr separierbar und eine geschlossene Lösung nicht mehr einfach möglich. Als eine Näherung wird der leicht lösbare Gleichgewichtsfall betrachtet, dass eine gleichmäßige Kraft unbegrenzt lange wirkt. Diese Kraft lässt sich dann schreiben als $f(x, t)=f_{0}$, wobei $f_{0}$ die 


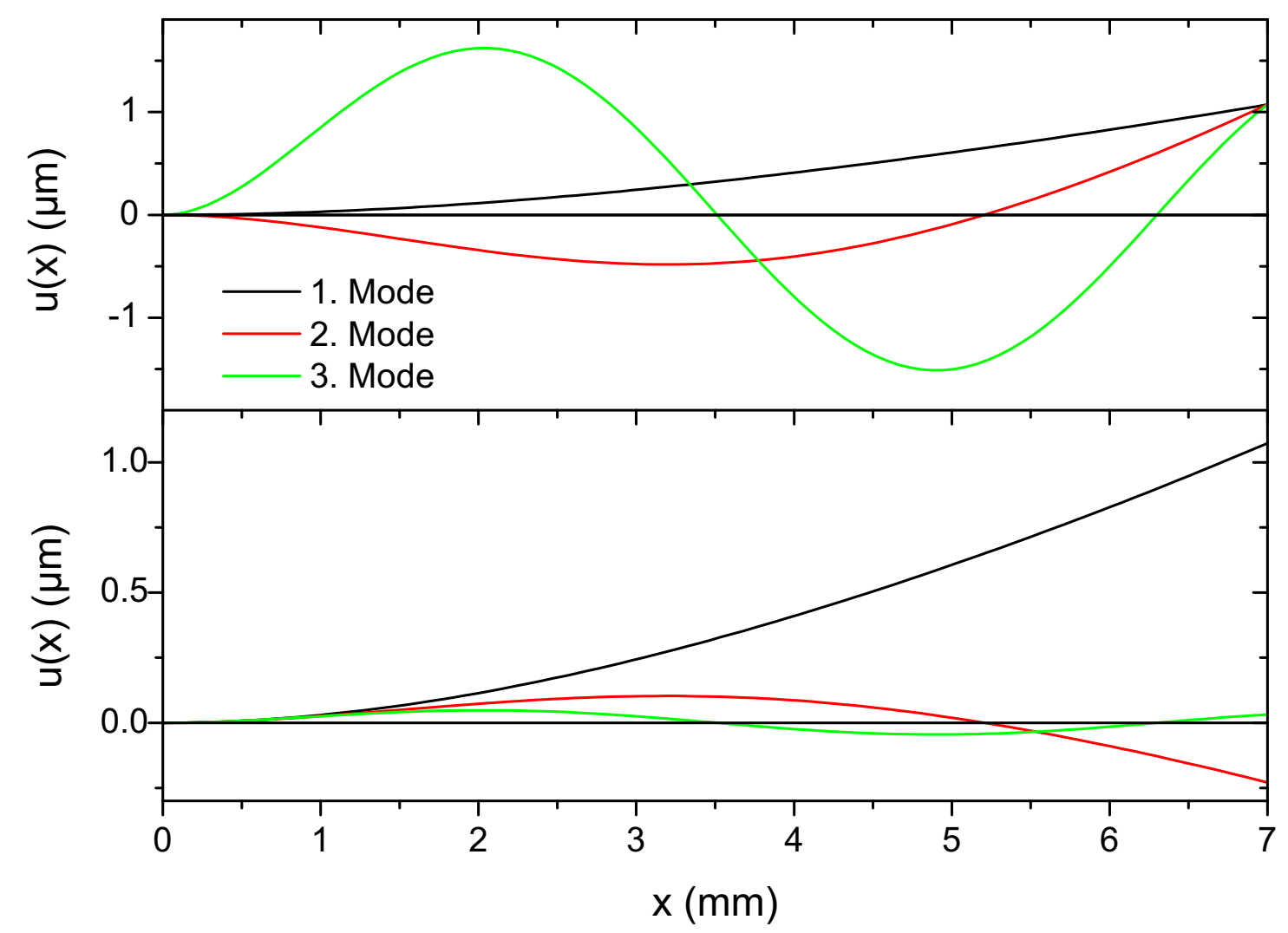

Abbildung 3.2: Biegelinie der ersten drei Schwingungsmoden; oben: normiert auf die Auslenkung an der Spitze; unten: normiert auf die Gesamtenergie (siehe 3.2.2)

Kraft pro Länge bezeichnet. Da eine zeitliche Abhängigkeit nicht betrachtet wird, bedeutet das, dass $E_{\text {kin }}$ verschwindet und $\mathfrak{L}\left(u, u^{\prime \prime}\right)$ kann geschrieben werden als

$$
\mathfrak{L}\left(u, u^{\prime \prime}\right)=-E_{\mathrm{pot}}-E_{\mathrm{f}}=\int_{0}^{l} d x\left(-\frac{k}{2} u^{\prime \prime 2}+f_{0} u\right) .
$$

Daraus folgt

$$
u^{\prime \prime \prime \prime}(x)=\frac{f_{0}}{k}
$$

was durch Integrieren die Lösung

$$
u(x)=\frac{f_{0}}{24 k} x^{4}+C_{3} x^{3}+C_{2} x^{2}+C_{1} x+C_{0}
$$


ergibt. Wiederum ergeben sich die Randbedingungen aus der eingespannten Seite und der Kräftefreiheit auf der offenen Seite:

$$
u(0)=u^{\prime}(0)=u^{\prime \prime}(l)=u^{\prime \prime \prime}(l)=0 .
$$

Daraus lassen sich die Parameter $C_{0}$ bis $C_{3}$ bestimmen und es folgt

$$
u(x)=\frac{f_{0}}{24 k} x^{2}\left(x^{2}-4 l x+6 l^{2}\right) .
$$

Diese Lösung ist nicht identisch mit der Biegelinie aus 3.5 mit den Parametern aus 3.7 . Die Anregung führt also immer dazu, dass gleich mehrere Schwingungsmoden angeregt werden. Dies wird in Abschnitt 3.2.2 diskutiert.

\subsubsection{Auslenkung durch das Plasma}

\section{Energie der Plasmakeule}

Die Auslenkung des Balkens erfolgt durch die Plasmakeule des PLD Prozesses mit einem Metall-Target (in diesem Fall Ag). Hier soll berechnet werden, wie groß diese Auslenkung maximal sein kann. Dazu wird zunächst die obere Grenze abgeschätzt, indem die Energie der Ionen aus der Plasmakeule bestimmt wird. Zuerst wird die Anzahl der auftreffenden Ionen berechnet. Nach Krebs et al. [61] ist die horizontale Verteilung durch eine $\varphi=$ $\cos (\varphi)^{24}$ Verteilung anzupassen, wohingegen in vertikaler Richtung eine $\cos (\psi)^{8}$ Verteilung passt. Dies ist in Abbildung 3.3 aufgetragen. Dazu wurde die Gesamtzahl der Teilchen nach Timm et al. [123] auf $1 \cdot 10^{15}$ normiert. Integrieren wir nun über den Raumwinkel im zentralen Bereich der mit einer Fläche von $2 \times 7 \mathrm{~mm}$ einem typischen Schwingungsbalken entspricht, so erhalten wir eine Teilchenzahl von $1 \cdot 10^{13}$, was sehr genau der üblichen Depositionsrate von $\sim 10 \mathrm{pm}$ pro Puls entspricht. Gehen wir jetzt weiterhin in einer oberen Abschätzung davon aus, dass alle Teilchen im Mittel eine Energie von $100 \mathrm{eV}$ haben, so erhalten wir eine Gesamtenergie durch den Puls von $1 \cdot 10^{15} \mathrm{eV}$ oder auch 0,16 mJ. Unter der Annahme, dass diese Energie völlig in die Auslenkung des Balkens umgesetzt wird, setzt man diese Energie als potentielle Energie in Gleichung 3.1 mit der Biegelinie für die Anregungsbiegung aus 3.8 ein und erhält als obere Abschätzung eine Anfangsauslenkung von etwa 1,1 mm, was sicher jenseits des hier betrachteten linearen Bereichs ist.

Es zeigt sich damit, dass nur ein Bruchteil der in der Plasmakeule vorhandenen Energie ausreicht, um den Balken zu gut messbaren Schwingungen anzuregen. 


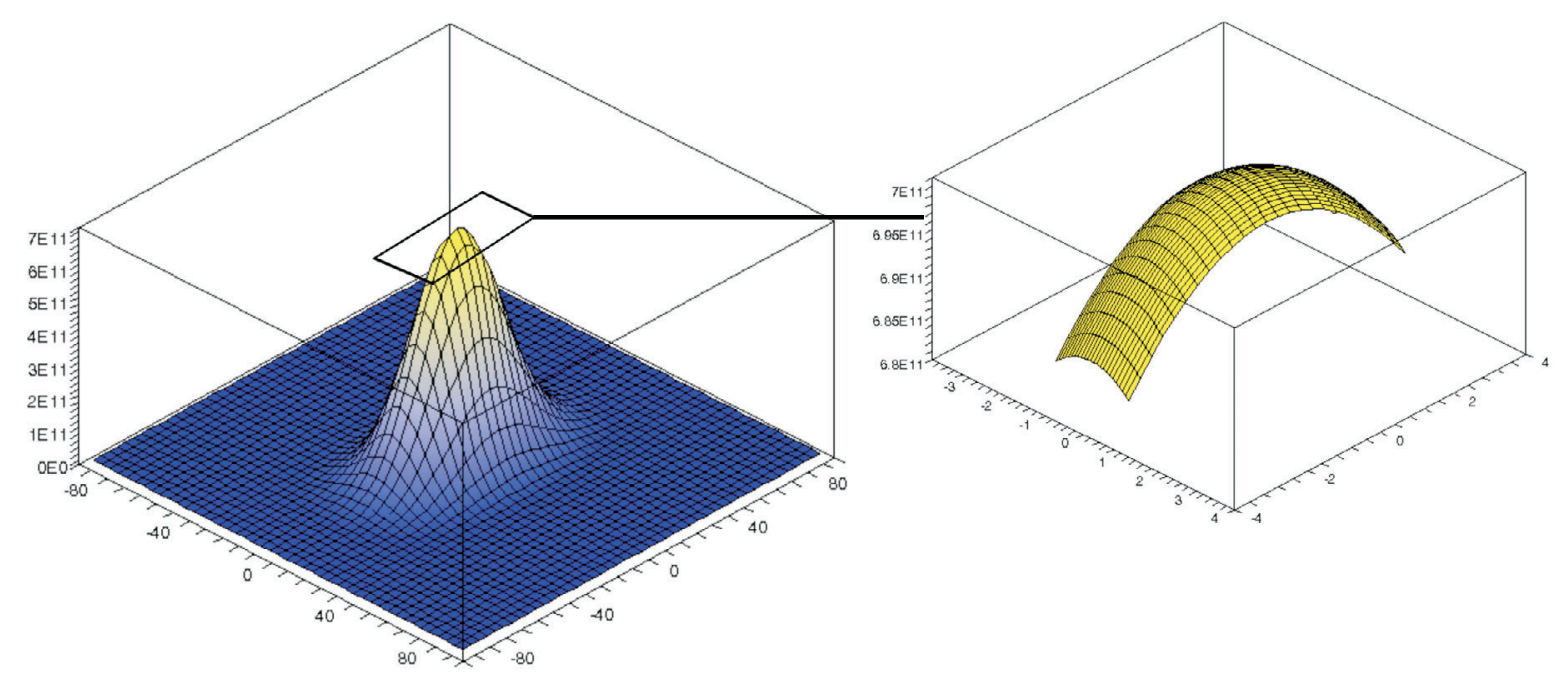

Abbildung 3.3: Teilchenzahlverteilung über dem Raumwinkel; rechts: Ausschnitt für die Fläche eines Substrats mit $7 \times 2 \mathrm{~mm}^{2}$ Größe

\section{Gemessene Auslenkung}

Experimentell läßt sich durch den Ausschlag des Lichtzeigers die Neigung des Substrats am Ort des Laserstrahls bestimmen. Da die Schwingung im kHz-Bereich liegt, ist es einfacher die Auslenkung während einer späteren Schwingung zu messen, als für die erste Biegung. Damit wird unter der Annahme der ersten Schwingungsmode der Gleichung 3.5 die Auslenkung berechnet. Wir berechnen mit einem Ausschlag des Lichtzeigers von 0,1 mm eine Auslenkung von etwa $1 \mu \mathrm{m}$. Dies liegt in einer sinnvollen und erwarteten Größe und bedeutet, dass nur ein geringer Teil der Plasmaenergie in die Schwingung geht, der größte Teil geht in die Bildung von Gitterfehlern oder in die Erwärmung des Substrats.

\section{Anregung unterschiedlicher Moden}

Wie in Abschnitt 3.2.1 gezeigt, ist die genäherte Auslenkung der Anregung (Gleichung 3.8) nicht identisch mit einer der Schwingungsmoden. Die ersten drei Schwingungsmoden und die Anregungsbiegung sind für die hier interessanten Parameter (Si-Substrat, $l=7 \mathrm{~mm}, b=$ $2 \mathrm{~mm}, d=100 \mu \mathrm{m}$ ) in Abbildung 3.2 aufgetragen. Es ist nun möglich diese Anregungsmoden nach den Eigenwerten der Schwingung, d. h. nach den verschiedenen Schwingungsmoden zu entwickeln. Wie man in Abbildung 3.4 sieht, reichen die ersten drei Moden mit numerisch bestimmten Parametern aus, um die Anfangsbiegung sehr gut zu nähern. Berechnet man nun aus dem Amplitudenverhältnis der ersten drei Moden die Energie, die in jeder dieser 


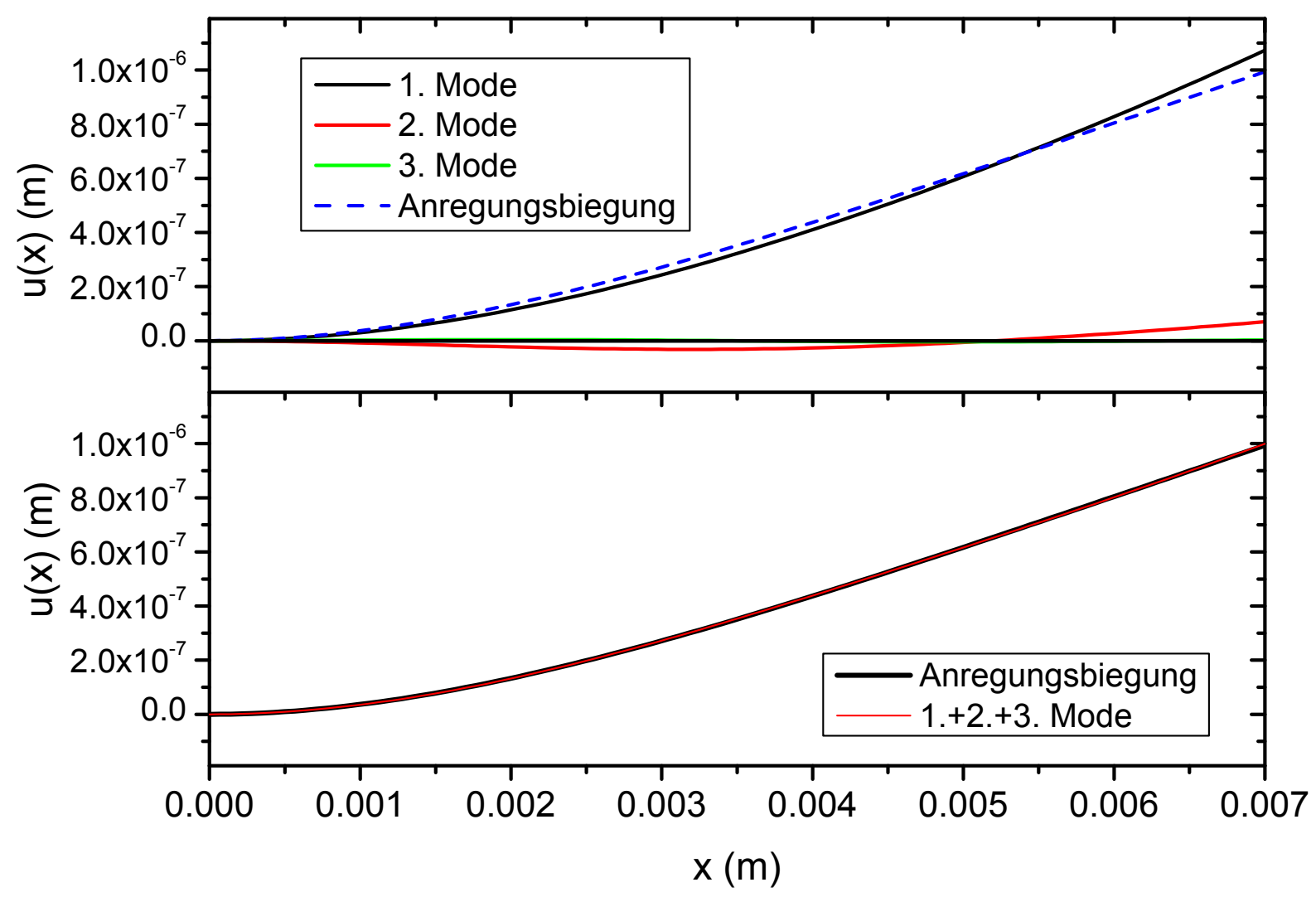

Abbildung 3.4: Zerlegung der Anregungsbiegung (Gleichung 3.8) in die Schwingungsmoden; oben: einzelne Schwingungsmoden; unten: schwarz: Anregungsbiegung, rot: Summe der ersten 3 Schwingungsmoden

drei steckt, so erhält man ein Verhältnis von etwa 1:10 (0,094) von der ersten zur zweiten und etwa 1:100 (0,0084) von der ersten zur dritten. Dies wurde sowohl für den Maximalfall mit der Gesamtenergie 0,16 mJ als auch für die gemessene Auslenkung berechnet und führt zu annähernd identischen Verhältnissen. Dieses starke Ungleichwicht bezüglich der Moden ist natürlich unter anderem dadurch bedingt, dass die angenommene Anfangsauslenkung des Balkens über die gesamte Länge in eine Richtung, d. h. weg vom einfallenden Plasma, erfolgt. Damit sollte sie grundsätzlich, wenngleich mit etwas anderen Zahlenwerten, auch gelten, wenn man über die vereinfachenden Annahmen, die in Abschnitt 3.2.1 gemacht wurden, hinaus geht. 


\subsection{Mechanischer Aufbau der Apparatur}

Kernstück einer vibrating reed Apparatur ist der einseitig eingeklemmte, schwingende Balken. Dieser besteht in diesem Fall aus (100) zur Oberfläche orientiertem, einkristallinem Silizium, da dieses eine sehr geringe Dämpfung bei gleichzeitig guter Verfügbarkeit und geringen Kosten aufweist. Aus beidseitig polierten Wafern der Dicke $100 \mu \mathrm{m}$ (CRYSTECH) wird der Balken nach Anritzen mit einem Diamantschneider gebrochen. Die Abmessungen liegen bei 1-2 mm Breite und je nach Frequenz 4-16 mm frei schwingender Länge plus ca. $2 \mathrm{~mm}$ für die Einspannung. Ein großes Länge zu Breite Verhältnis ist wichtig, damit eine Querverbiegung möglichst wenig Einfluss hat [47]. Das Brechen erfolgt so, dass in alle Hauptrichtungen des Balkens eine $\{100\}$-Richtung des Kristalls zeigt.

Da die Einspannung des Balkens der kritische Punkt für die Energiedissipation und somit die Dämpfung ist, muss diese besondere Anforderungen erfüllen. Hier wird der SiBalken in eine Halterung aus Aluminium eingespannt. Al verfügt über eine hohe Wärmeleitfähigkeit bei gleichzeitig geringer Wärmekapazität, was für die Verbindung zwischen dem Heizofen und dem zu heizenden Substrat beim Wechsel von Temperaturen sehr gut geeignet ist. Die Form des Aluminiumhalters wurde speziell so entworfen, dass die Einklemmung über drei Schrauben erfolgt und die Verbindungslinie zwischen zwei der Schrauben genau auf der vorderen Einklemmkante liegt (Abb. 3.5. Auf diese Weise ist die Einklemmkraft genau auf dieser Linie optimal einzustellen und diese Line, bei der auch die maximale Kraft durch die Schwingung auftritt, liegt an der vordere Kante. Wirkt die stärkste Kraft weiter hinten, wird die Dämpfung stark erhöht. Der Schwingungsbalken wird in den speziell
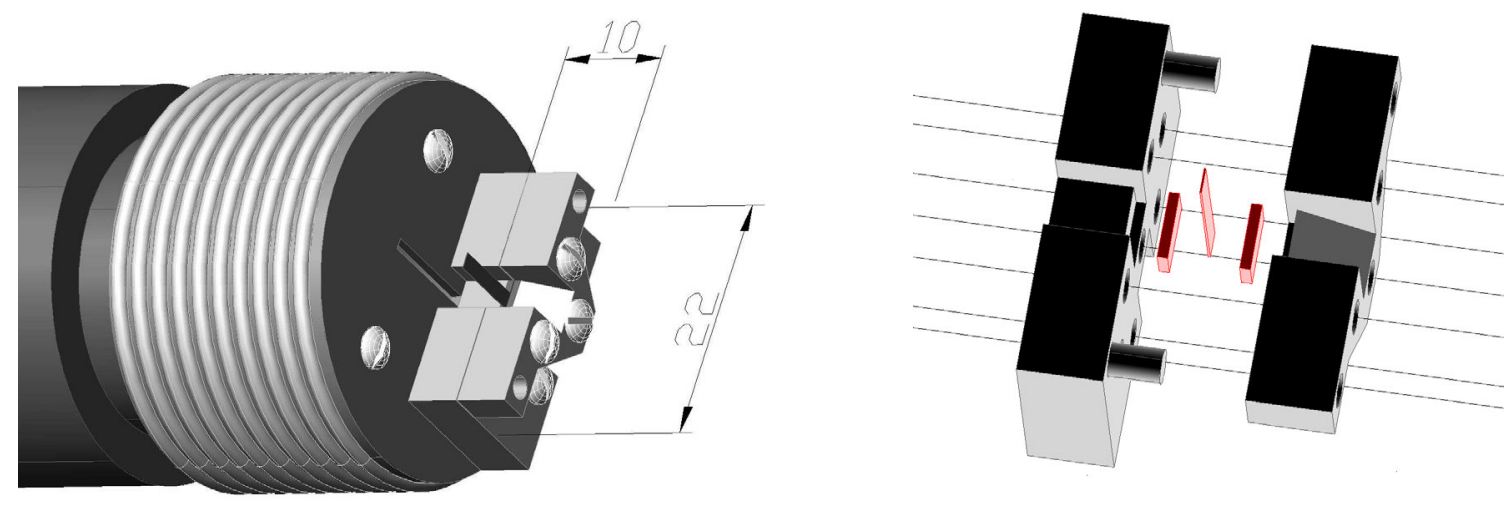

Abbildung 3.5: Links: PPXR-Aufbau mit Ofen und schwingender Einheit; Rechts: Explosionszeichnung zum Verdeutlichen der Einspannung in Si-Blöcke (rot)

geformten Al-Block zwischen zwei $650 \mu \mathrm{m}$ dicke Si-Plättchen geklemmt, die in das Al einge- 
lassen sind (Abb. 3.5 rechts). Auf diese Weise erfolgt die kritischste Stelle der Einspannung durch ein sehr hartes, einkristallines Material und kann nach jedem Versuch ausgewechselt werden, was eine langfristige Beschichtung der Einspannungsstelle verhindert. Da hier die größten Kräfte auftreten, würde dies die Messung maximal stören. Die Wärmeleitfähigkeit von $\mathrm{Si}$ ist immer noch doppelt so hoch wie die von Eisen und damit ausreichend. Diese $4 \times 2 \times 0,65 \mathrm{~mm}^{3}$ großen Si-Plätchen werden aus einem einseitig polierten Si (111)-Wafer gebrochen und anschließend nass auf einer SiC-Schleifscheibe exakt auf die passenden Dimensionen poliert. Dabei ist darauf zu achten, dass die vorderen Einklemmkanten präzise aufeinander liegen, da sonst die Güte der Einspannung deutlich reduziert wird. Eine gute Einspannung ist erreicht, wenn man durch Streichen mit einer feinen Spitze quer über die beiden eingespannten Si-Plättchen keinen Spalt mehr fühlen kann. Um den Effekt der unterschiedlichen thermischen Ausdehnung von Silizium, Aluminium und Stahlschrauben auszugleichen, wurden unter den haltenden Schrauben sehr steife Stahlfedern befestigt (nicht gezeigt in Abb. 3.5), die die Halterung auch bei höheren Temperaturen festhalten.

Der beschriebene Al-Block ist unter dem Boden einer becherförmigen Halterung aus Kupfer befestigt, die fest von Mantelheizleitern umgeben ist. Im Boden diese Heizofens befindet sich eine Blende um einen Laserstrahl passieren zu lassen (siehe Abb. 3.5). Der $\mathrm{Cu}$-Becher ist mit drei Schrauben, auf Federn gelagert, an einem stabilen Edelstahlrohr befestigt, das an einem Kammerflansch sitzt. Eine technische Skizze der Gesamtapparatur ist in Abbildung 3.6 zu sehen. Auf diese Weise ist der gesamte heizbare Aufsatz in allen

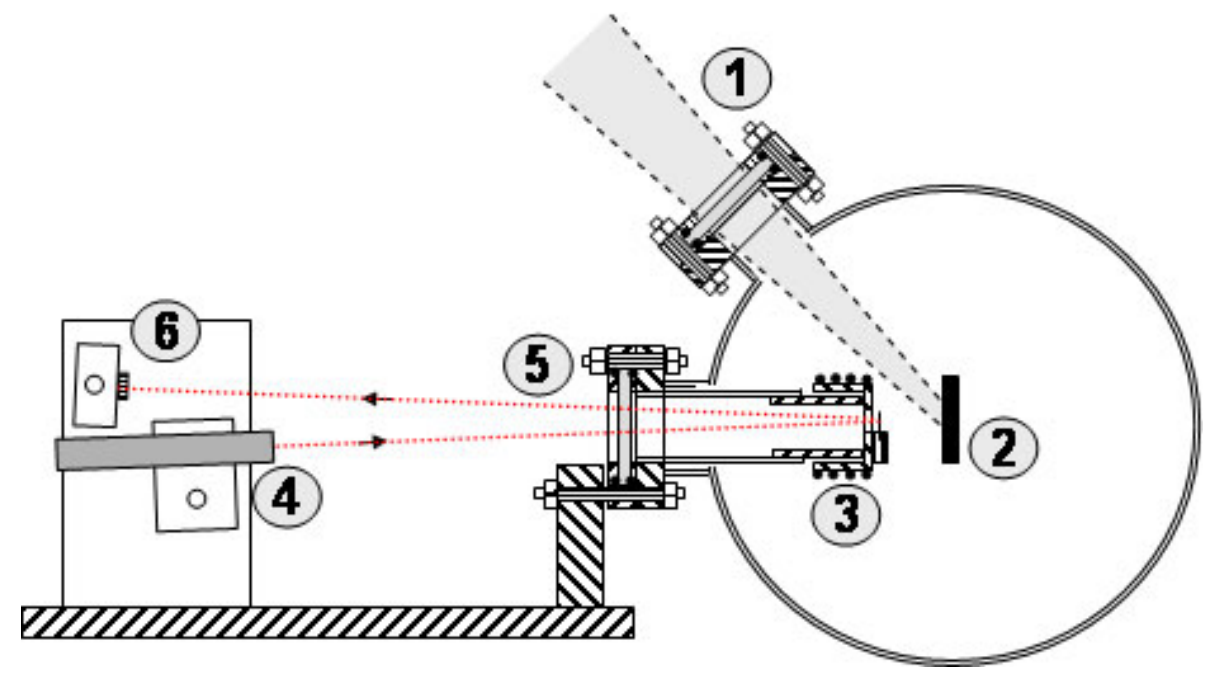

Abbildung 3.6: Schematischer Aufbau der PPXR-Apparatur: 1: Eintrittsfenster für UVLaser; 2: Target; 3: PPXR-Messkopf mit Ofen (Abb. 3.5); 4: Diodenlaser; 5: Eintrittsfenster für Messlaser; 6: Detektor 
drei Kipprichtungen justierbar, was für die Messung mit dem Laserstrahl wichtig ist. Die Wärmeableitung durch die drei Schrauben ist sehr gering, der Ofen läßt sich mit nur $5 \mathrm{~W}$ Leistung leicht auf $250{ }^{\circ} \mathrm{C}$ heizen, und am Kammerflansch läßt sich auch nach einigen Stunden nicht die geringste Erwärmung feststellen. Dies ist sinnvoll, da sich dadurch die Apparatur nicht verzieht und der Laserstrahl aus dem Detektorbereich wandert. Die Genauigkeit und Reproduzierbarkeit der Temperatur am Substrat wird für Temperaturen bis $250{ }^{\circ} \mathrm{C}$ mit schlechtestenfalls $5{ }^{\circ} \mathrm{C}$ abgeschätzt.

Durch die Halterung aus $\mathrm{Al}$ ist es ausgeschlossen, dass $\mathrm{Cu}$ bei erhöhten Temperaturen in das Siliziumsubstrat eindiffundiert. In dem Al-Block befindet sich weiterhin eine Bohrung, in die ein Ni-CrNi Mantelthermoelement mit $1 \mathrm{~mm}$ Durchmesser(ThermocoAx) eingeführt wird, um die Temperatur möglichst nah an der Probe zu messen. Die Temperatur wird über einen PID-Regler (Eurotherm 902S) geregelt. Dieser steuert über den analogen Ausgang die Stromquelle (Heinzinger Polaris 35-20) und damit die Heizung über die Mantelheizleiter. Diese Heizung wurde hier nur bis zu $350{ }^{\circ} \mathrm{C}$ genutzt, apparative Einschränkungen ergeben sich aber erst durch das Schmelzen des Aluminiumhalters oberhalb von $600{ }^{\circ} \mathrm{C}$.

Mit dem hier verwendeten PPXR ist die untere Grenze der messbaren Frequenz durch die maximal sinnvoll zu präparierende Länge des Substrats von ca. $16 \mathrm{~mm}$ mit etwa $500 \mathrm{~Hz}$ gegeben. Die obere Grenze der Messfrequenz wird durch die Samplingrate der verwendeten Wandlerkarte begrenzt (siehe Abschnitt 3.4). Bei 7,5 kHz erhält man nur etwa drei Messpunkte pro Halbschwingung. Diese Grenze ließe sich durch eine andere Karte leicht verschieben, allerdings beträgt die Länge hier nur $4,25 \mathrm{~mm}$, was die praktische Anwendung schon begrenzt.

\subsection{Elektronische Messwerterfassung}

Der elektronische Messaufbau liegt außerhalb der Vakuumkammer (Erkennbar in Abb. 3.6). Ein Diodenlaser trifft durch ein Flanschfenster und die Blende in dem Cu-Halter auf die Rückseite der Probe. Der reflektierte Strahl tritt wieder durch das Fenster und trifft auf einen in zwei Dimensionen justierbaren positionsempfindlichen Detektor (Position Sensitive Detector, PSD, SiTek 1L10 PSD). Dieser wandelt die Position auf die der Strahl auftrifft mit hoher räumlicher Auflösung und einer Reaktionszeit von etwa 20 ns linear in ein Stromsignal um. Dieses Signal wird in ein Spannungssignal umgewandelt, verstärkt und über gut abgeschirmte Kabel in den line-in-Eingang einer handelsüblichen AD-DA-Wandlerkarte 
(Creative Sound Blaster AudioPCI 128) mit einer Samplingrate von $44.100 \mathrm{~Hz}$ zur rechnergestützten Erfassung weitergeleitet. Durch diese Elektronik wird fast keine elektronische Filterung vorgenommen, nur ein Hochpassfilter der Wandlerkarte bei $23 \mathrm{~Hz}$ schneidet die Gleichstromanteile ab.

\subsection{Ablauf des Messverfahrens}

Die gesamte Messung erfolgt rechnergesteuert über ein selbstentwickeltes Java-Programm. Ein vereinfachtes Flussdiagramm des Messablaufs ist in Abbildung 3.7 gezeigt. Aus einer Steuerdatei werden die anzusteuernden Temperaturen gelesen und per RS232 Schnittstelle

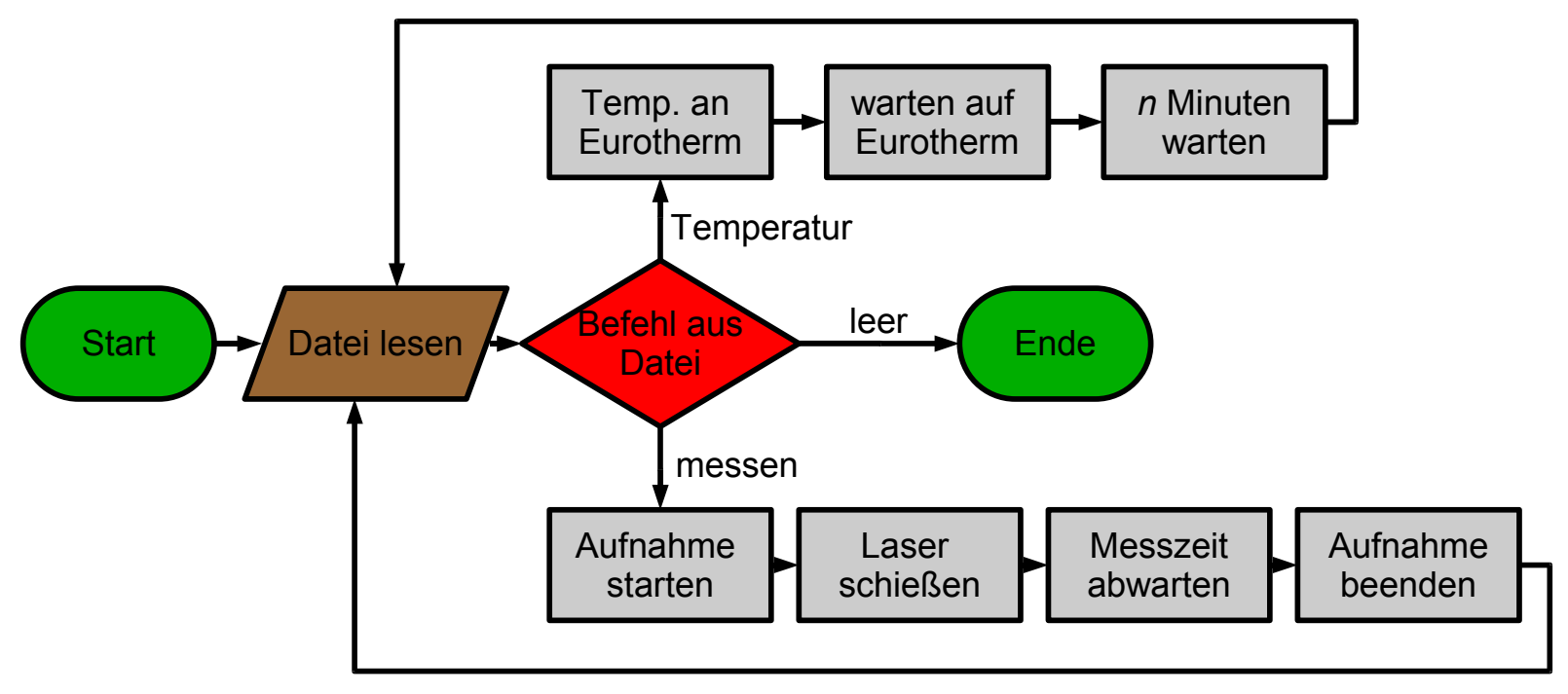

Abbildung 3.7: Vereinfachtes Flussdiagramm des Messablaufs

an den Eurothermregler gesandt. Ist die Temperatur am Thermoelement erreicht, wird sie eine voreingestellte Zeit zwischen einer und fünf Minuten gehalten, wobei in Versuchen keine messbare Abhängigkeit zwischen der Haltezeit und der Qualität der Messung festgestellt werden konnte. In dieser Zeit kann sich im System das thermische Gleichgewicht einstellen. Danach wird die Aufnahme an der Wandlerkarte gestartet und über eine zweite RS232 Schnittstelle ein Signal an die Lasersteuerung gegeben, um einen Puls abzugeben und die Plasmakeule zur Anregung zu erzeugen. Nach der vorgewählten Messzeit wird die Aufnahme beendet und die Daten werden im wav-Format binär abgespeichert. Eine sinnvolle Messzeit ist abhängig von der gewählten Schwingungsfrequenz und der Dämpfung 
und liegt zwischen $2 \mathrm{~s}$ für $7,5 \mathrm{kHz}$ und hoher Dämpfung und $10 \mathrm{~s}$ für das reine Substrat bei $500 \mathrm{~Hz}$. Es wurde versucht, die Messzeit so kurz wie möglich zu halten, da die Daten viel Speicherplatz benötigen und der Aufwand für die Auswertung (Abschnitt 3.6) mit $n \log _{2}(n)$ überproportional steigt.

\subsection{Auswertung der Messung}

Auch die Auswertung erfolgt über ein selbstentwickeltes Java-Programm. Hierzu werden die Daten aus dem speichersparenden wav-Format gelesen und gegen die Zeit aufgetragen.

Eine typische Messung ist im oberen Teil der Abbildung 3.8 gezeigt. Da die Messungen,

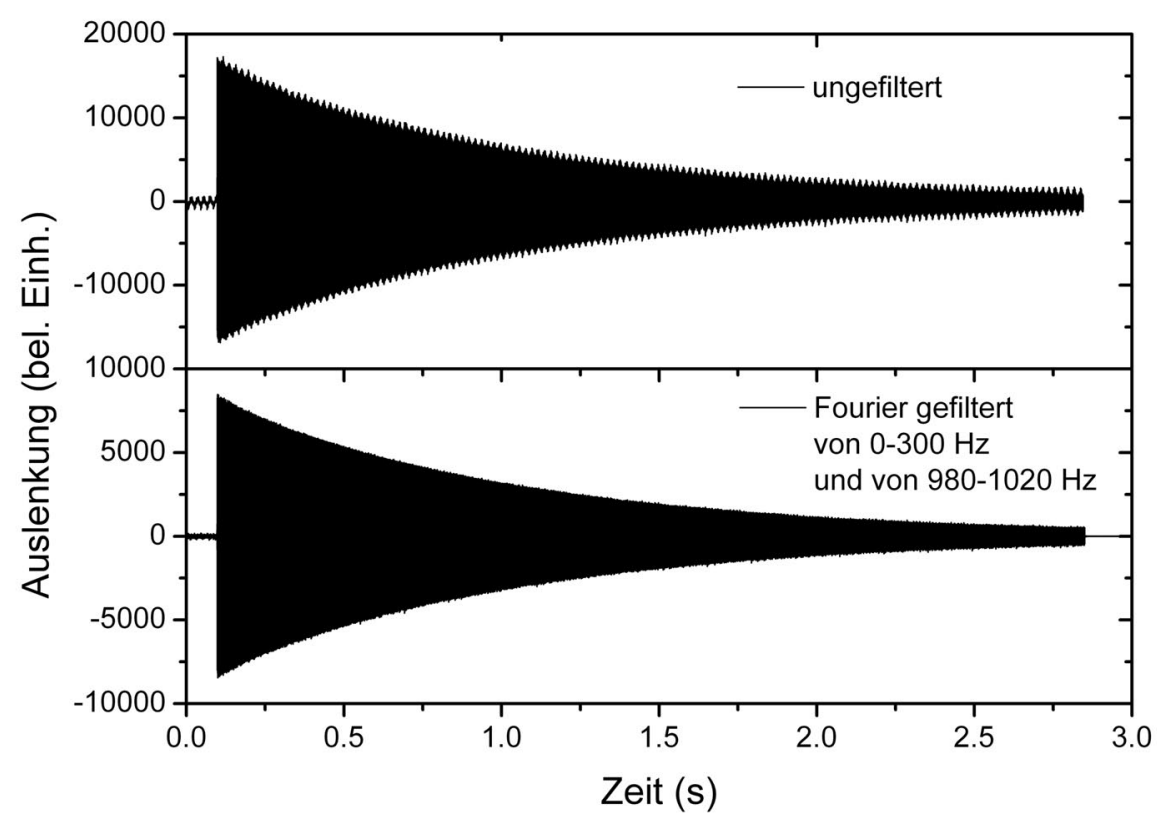

Abbildung 3.8: Typische vibrating reed Messungen mit PPXR. Oben: Original Messdaten; Unten: Fourier-gefiltert

wie deutlich sichtbar ist, teilweise noch von einem Rauschen überlagert werden, ist es nicht sinnvoll möglich, einfach die Maxima der Sinusschwingung zu nehmen, um daraus das logarithmische Dekrement zu bestimmen. Stattdessen wird die gemessene Kurve zuerst durch einen variablen Fourier-Filter gefiltert, was das Ergebnis deutlich verbessert, wie im unteren Teil der Abbildung zu sehen ist. Auf diese Weise werden physikalisch nicht sinnvolle Schwingungen, wie die $50 \mathrm{~Hz}$ des Stromnetzes und die $1000 \mathrm{~Hz}$ der Turbomolekularpumpen entfernt. Diese Filterung bewirkt nichts anderes als ein elektronisches Filter, wird hier 
jedoch sehr viel vorsichtiger eingesetzt als beispielsweise bei der Verwendung eines lock-in Verstärkers. Im Gegensatz zu elektronischen Lösungen kann die mathematische zusätzlich flexibel auf jede Messung angepasst werden und ermöglicht beliebig scharfe Filterwahl.

Da auch die gefilterte Funktion noch ein leichtes Rauschen zeigt, wird die Präzision erhöht, indem nicht nur die Maxima, sondern alle Messdaten für die Auswertung verwendet werden. Dafür wird folgender Algorithmus verwendet: Es werden für jede halbe Sinusschwingung die maximalen Messwerte gesucht. Von diesen aus werden zu kleineren und größeren Zeiten hin alle Werte bis hinter dem Nulldurchgang einbezogen. An diese Halbschwingung wird eine Sinusschwingung $A_{0} \sin (\omega t+\varphi)$ mit der Methode der kleinsten Quadrate angepasst. Dies ist in Abbildung 3.9 gezeigt.

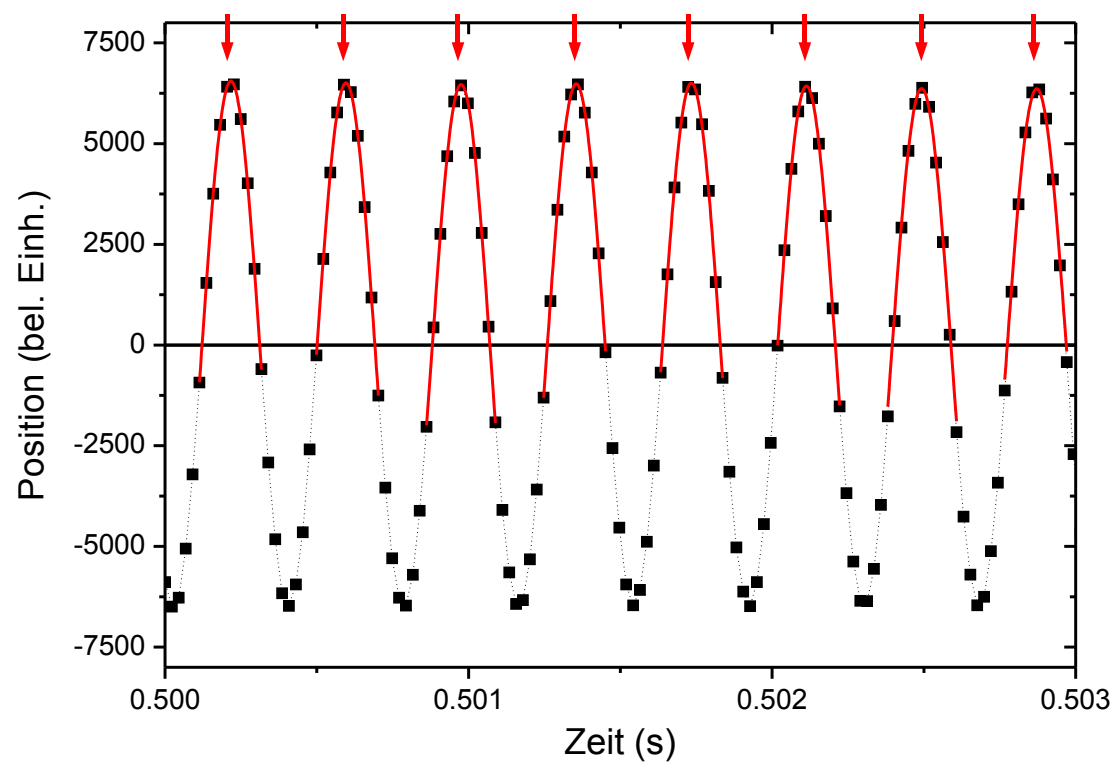

Abbildung 3.9: Ausschnitt aus einer PPXR-Messkurve mit angepassten Halbschwingungen (siehe Text)

Es wird ein einfacher Newtonscher Optimierungsalgorithmus verwendet, was sehr gut ausreicht, da die Anfangswerte schon sehr genau erraten werden können. Die Amplitude $A_{0}$ passt mit wenigen Prozent Abweichung mit der Höhe des höchsten Messwertes überein, die Phasenlage $\varphi$ bestimmt sich durch lineare Interpolation zwischen den beiden ersten Punkten, wo die Krümmung des Sinus gegen Null geht. Die Schwingungsfrequenz $\omega$ folgt aus der vorher erfolgten Fourier-Transformation mit einer Genauigkeit im Bereich von $10^{-4}$ und wird zur Anpassung nicht verändert. Die Einschränkung der Genauigkeit ist hier im 
Wesentlichen durch die Auflösung in der Fourier-Transformierten (bestimmt durch fast fourier transformation, FFT) und damit durch die Anzahl an Datenpunkten bestimmt. Um diese zu erhöhen, wurde eine zusätzliche Nullauffüllung (zero filling) um einen Faktor 4 vorgenommen. Auf diese Weise berechnet sich für jede einzelne Halbschwingung die Höhe des Maximums befreit von hochfrequentem Rauschen, und die Position auf der Zeitachse ist deutlich genauer als der Abstand zwischen zwei Messwerten.

Dieses Verfahren beginnt automatisch bei dem absoluten Maximum der Messung. Dies ist das erste Maximum nach dem Plasmapuls. Einbezogen werden Halbschwingungen, bis die Maxima einen Zeitraum von 1/100 Sekunde nicht mehr über 30\% des ersten Maximums steigen. Dieser Wert wurde empirisch als sinnvoll bestimmt, er liegt noch weit von dem Hintergrundrauschen entfernt und läßt auch mit mehreren tausend auszuwertenden Halbschwingungen genug Statistik.

Die so erhaltenen Maxima werden als nächstes einem Ausreißertest unterzogen. Weichen sie mehr als 10\% von ihren beiden Vorgängern ab, so werden sie einfach gestrichen. Dies ist notwendig, da durch die automatische Auswahl der Punkte und das Fit-Verfahren immer wieder falsche Werte gefunden werden. Diese liegen jedoch in der Regel bei weit unter einem Prozent der gefundenen Maxima und können damit gut vernachlässigt werden.

An die Logarithmen der so bereinigten Werte wird nun eine Gerade angepasst. Dieses Verfahren bietet sich an, da eine lineare Anpassung nach der Methode der kleinsten Quadrate einfach, exakt lösbar ist (Abb. 3.10). Das komplette Verfahren wird genauso für die negativen Halbschwingungen durchgeführt. Auf diese Weise ergeben sich zwei Geraden, die (abgesehen vom Vorzeichen) die gleiche Steigung haben sollten. Ist dies nicht der Fall ist dies ein gutes Indiz dafür, dass es sich um eine Fehlmessung handelt.

Dieses automatisierte Verfahren ist sehr wenig störanfällig, jedoch kommt es in der Größenordnung von etwa einem von hundert Fits zu fehlerhaften Anpassungen, die meist durch fehlerhafte Messungen begründet werden. In diesem Fall liegen die Maxima deutlich außerhalb der anderen Werte und lassen sich leicht mit einem Ausreißertest entfernen. Dieser wurde so programmiert, dass die Ausreißerschwelle frei wählbar und damit auf die gemessene Kurve anpassbar ist.

\subsubsection{Alternative Auswertung im Fourier-Raum}

Eine Alternative für die komplexe Auswertung ist die Analyse der Dämpfung direkt im Fourier-Raum. Diese ist beispielsweise für NMR-Messungen üblich, jedoch soll hier gezeigt werden, dass sie für die hier durchgeführten Messungen nicht sinnvoll ist. Die Fourier- 


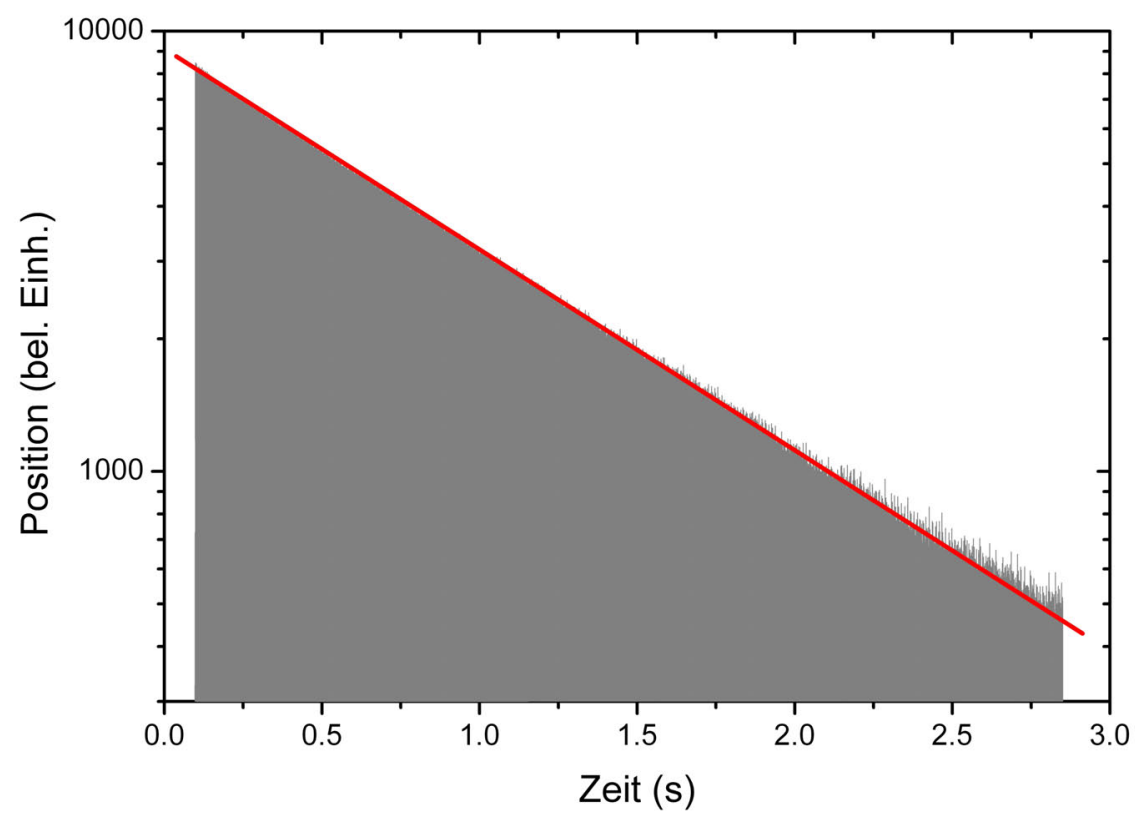

Abbildung 3.10: Messkurve aus Abbildung 3.8 in logarithmischer Auftragung mit angepasster Gerade

Transformierte der gedämpften Schwingung $\mathrm{e}^{-\Lambda t} \cos \left(\omega_{0} t\right)$ ist eine Lorentz-Kurve der Form $\frac{\Lambda}{\Lambda^{2}+\left(\omega-\omega_{0}\right)^{2}}$, wobei die Halbwertsbreite (FWHM) $2 \Lambda$ entspricht. Damit ist die Bestimmung der Dämpfung denkbar einfach. Nehmen wir als Beispiel die Messung aus dem letzten Abschnitt, so hat diese eine Dämpfung von $1,33 \cdot 10^{-5}$ pro gemessener Linie $(1 / 44100 \mathrm{~s})$. Dies bedeutet jedoch, dass die Halbwertsbreite in der Fourier-Transformierten nur 1,76 Linien beträgt und damit nicht auszuwerten ist. Eine Verbesserung ergibt sich durch Nullauffüllung (zero filling), d.h. die Rohdaten werden am Ende mit Nullen aufgefüllt um mehr Daten zu erhalten und in der fast fourier transform fft die Feinfechselwirkung besser herauszuheben.

Die Fourier-Transformierte, erhalten mit einem zero-filling-Faktor 4, wie er auch für die andere Auswertung zur Verbesserung der Frequenzauflösung verwendet wurde, ist in Abbildung 3.11 zu sehen, im Inset ist der Ausschnitt um die Frequenz der ersten Schwingungsmode vergrößert. Die berechnete Halbwertsbreite beträgt hiermit 7,0 fft Kanäle, eine Anpassung einer Lorentz-Kurve ergibt immer noch eine Abweichung in der Dämpfung von etwa $17 \%$.

Neben der stufigen Kurvenform ist zu sehen, dass die Fourier-Transformierte von einer Schwingung überlagert wird. Diese ist in den Abschneideeffekten durch eine endliche Messung begründet und ist in diesem Fall besonders stark, da eine im Verhältnis zur Abklingzeit 


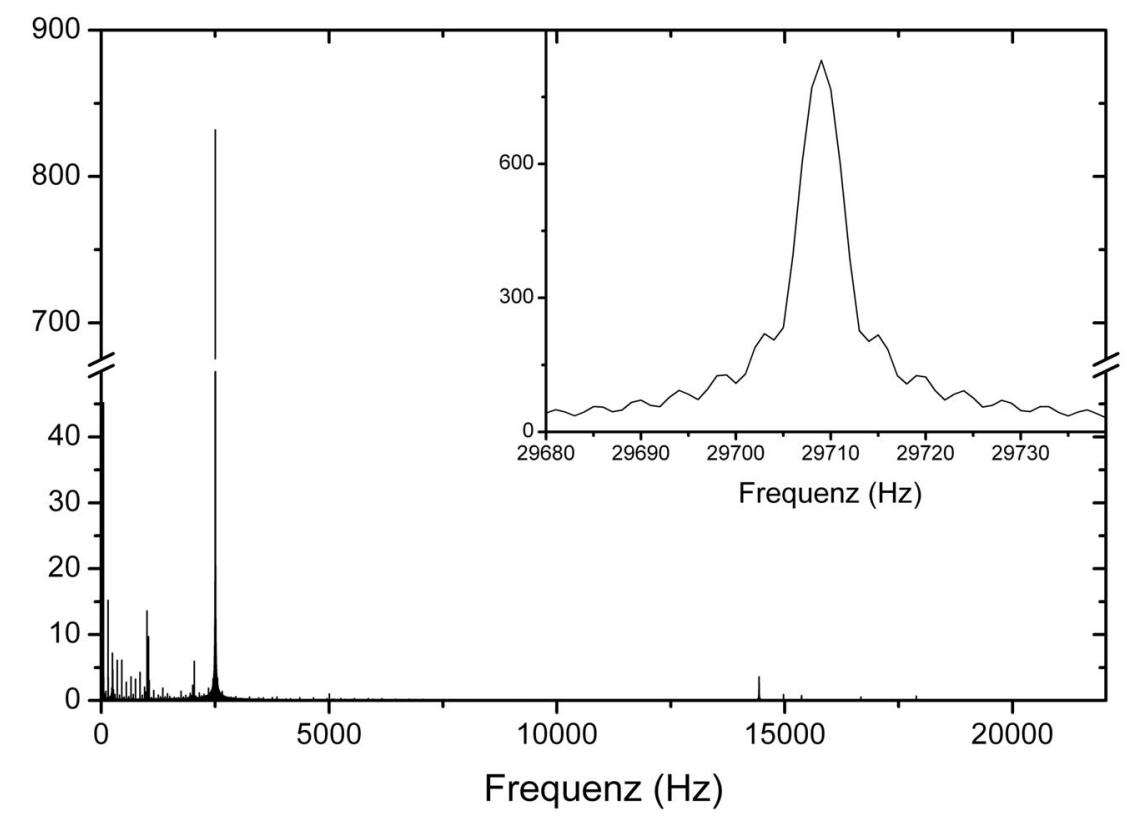

Abbildung 3.11: Fourier-Transformierte einer Messkurve; Inset: Vergrößerung um die Frequenz der ersten Schwingungsmode

kurze Messzeit gewählt wurde. Ein Versuch, das Ergebnis mit einem zero-filling-Faktor von $16 \mathrm{zu}$ verbessern, ist in Abbildung 3.12 gezeigt. Zusätzlich ist die Lorentz-Kurve berechnet mit der Dämpfung, die aus der vorherigen Auswertung im Realraum bestimmt wurden und eine im Fourier-Raum angepasste Lorentz-Kurve gezeigt. Es ist zu sehen, dass sich die beiden Kurven deutlich unterscheiden, also durch die überlagerte Schwingung eine korrekte Anpassung nicht möglich wäre. Der Fehler in der Dämpfung betrüge hier immer noch 13\%. Eine Verbesserung ist möglich, indem die Fehler durch die Abschneideeffekte durch ein Behandeln der endliche Messung mit einer Abschneidefunktion (Fensterung) reduziert werden. Da aber schon ein zero-filling um den Faktor 16, das für eine ausreichende Präzision notwendig ist, die verfügbare Rechenkapazität übersteigt, wurde dieser Ansatz nicht weiter verfolgt.

\subsection{Berechnung des komplexen Elastizitäts-Moduls}

Für die Auswertung und Bestimmung des komplexen E-Moduls der Filme liegen die gemessenen Frequenzen $\omega=2 \pi f$ und logarithmischen Dekremente $\Lambda$ des Substrats und des Systems Substrat+Film in Abhängigkeit von der Temperatur vor, wie sie durch das in Abschnitt 3.6 beschriebene Verfahren gewonnen wurden. Im Folgenden soll durchgehend 


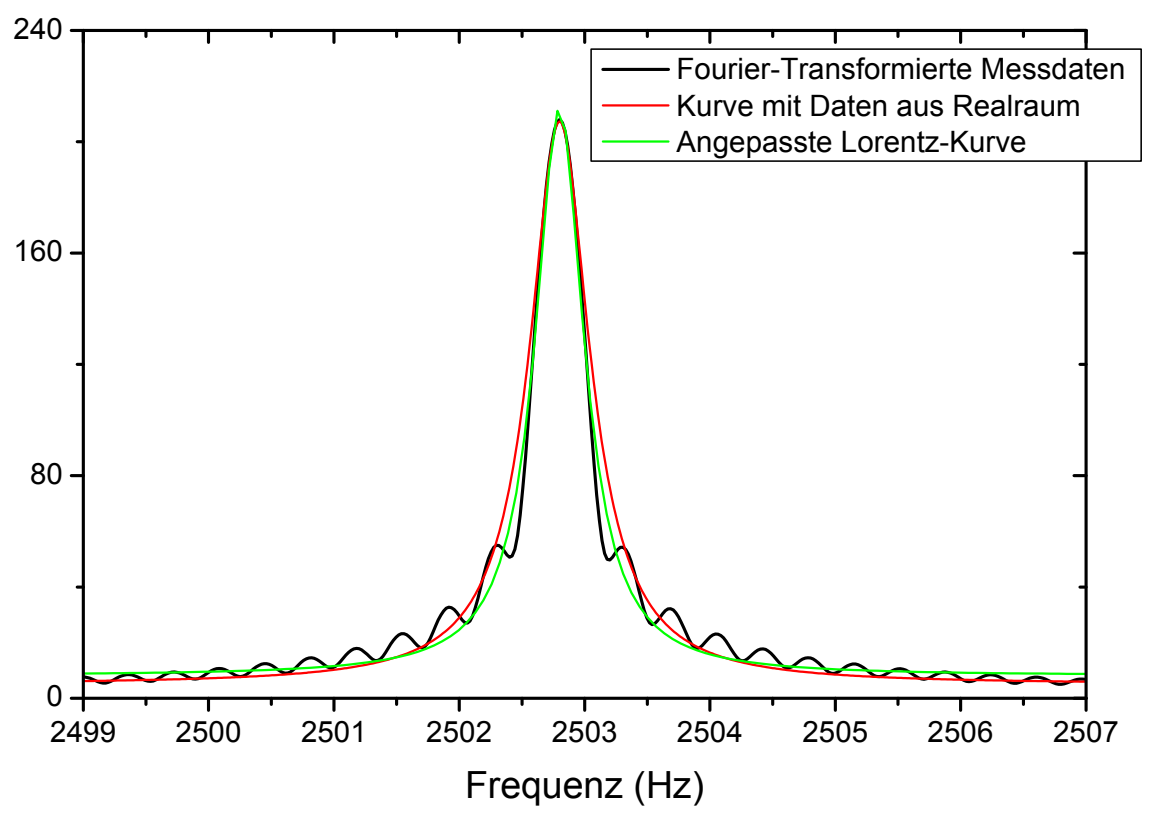

Abbildung 3.12: Fourier-Transformierte einer Messkurve mit zero-filling-Faktor 16 um die Frequenz der ersten Schwingungsmode mit der Lorentz-Kurve aus der im Realraum nach Abschnitt 3.6 ermittelten Dämpfung und einer im Fourier-Raum angepassten Kurve

der Index $s$ für das Substrat und der Index $f$ für den Film verwendet werden, sowie $s+f$ für das System Substrat+Film. Hier soll beschrieben werden, wie aus den vorhandenen Werten der komplexe E-Modul bestimmt wird. Dafür wird es notwendig sein, zuerst einige weitere Größen zu bestimmen, die Biegesteifigkeit und den E-Modul des Substrats sowie die Schichtdicke.

Aus Literaturdaten für den E-Modul des Substrats $E_{s}$ lässt sich zunächst aus Gleichung 3.2 die Biegesteifigkeit des Substrats $k_{s}$, die gespeicherte Energie pro Länge und Krümmung bestimmen:

$$
k_{s}=\frac{b d^{3} E_{s}}{12} .
$$

In diesem Fall liegt die Breite $b$ bei 1-2 mm und die Dicke $d$ bei $100 \mu \mathrm{m}$. Da die Literaturangaben für $E_{s}$ mit $165 \mathrm{GPa}$ [50] bei Raumtemperatur gemessen sind, nehmen wir diese für die niedrigste verwendete Temperatur, in diesem Fall $60{ }^{\circ} \mathrm{C}$ (Dieser Parameter geht nur in den absoluten Fehler ein und der Fehler durch die Temperatur wird hier mit etwa $1 \%$ abgeschätzt). 
Weiterhin lässt sich, immer noch bei der tiefsten Temperatur, durch Umstellen von Gleichung 3.6 der temperaturunabhängige Parameter $\gamma^{4}$ bestimmen

$$
\gamma^{4}=\frac{\rho}{k} \omega^{2}
$$

Dabei wird die Masse pro Länge $\rho$ aus der Literaturdichte für Si von $2230 \mathrm{~kg} / \mathrm{mol}$ errechnet. Zur Erinnerung sei darauf hingewiesen, dass für die Grundmode $\gamma l=1,8751$ gilt, so dass sich an dieser Stelle die Länge $l$ des Substrats berechnen lässt, was zur Kontrolle dient, und wesentlich genauer ist als die direkte Messung.

Mit diesem Ergebnis kann nun für das gesamte Temperaturintervall die Biegesteifigkeit $k$ des Substrates mittels

$$
k_{s}(T)=\frac{\rho_{s}}{\gamma^{4}} \omega_{s}^{2}(T)
$$

bestimmt werden. Daraus folgt dann mit Gleichung 3.2 der E-Modul des Substrats:

$$
E_{s}^{\prime}(T)=\frac{12}{b d^{3}} k_{s}(T)
$$

Als nächster Parameter wird die Filmdicke benötigt. Nimmt man an, dass der E-Modul des Films für Temperaturen deutlich über $T_{g}$ verschwindet $\left(E_{f}^{\prime} \rightarrow 0\right.$ für $\left.T \gg T_{g}\right)$, so verschwindet auch die durch die Krümmung im Film gespeicherte Energie und es gilt $k_{s}=k_{s+f}$ und damit

$$
\rho_{f}=\rho_{s+f}-\rho_{s}=k_{s} \gamma^{4}\left(\frac{1}{\omega_{s+f}^{2}}-\frac{1}{\omega_{s}^{2}}\right),
$$

was wiederum aus Gleichung 3.6 folgt. Die Filmdicke wird also bei der höchsten gemessenen Temperatur, hier i. A. $200-220^{\circ} \mathrm{C}$ bestimmt. Damit berechnet sich die Schichtdicke zu

$$
d_{f}=\frac{\rho_{f}}{\rho_{0} b}
$$

mit der tatsächlichen Dichte des Films $\rho_{0}$, die mit $1200 \mathrm{~kg} / \mathrm{m}^{3}$ angenommen wurde.

Die Biegesteifigkeit des Systems Balken und Schicht ergibt sich nun abhängig von der Temperatur durch

$$
k_{s+f}(T)=\frac{\rho_{s+f}}{\gamma^{4}} \omega_{s+f}^{2}(T) .
$$

Dabei wird angenommen, dass die Masse des Substrats und des Films sowie deren Länge nicht von der Temperatur abhängen und damit $\rho_{s+f}$ und $\gamma^{4}$ konstant sind. 
Integriert man nun die Biegesteifigkeit über den Film $\left(k_{f}=b \int_{d_{s} / 2}^{d_{s} / 2+d_{f}} d z z^{2} E_{f}^{\prime}\right)$ (von der Oberkante des Substrats $d_{s} / 2$ bis zur Oberkante des Films $d_{s} / 2+d_{f}$ ), so erhält man für den Speichermodul und Realteil des komplexen E-Moduls des Films die Abhängigkeit

$$
E_{f}^{\prime}=\frac{k_{f}}{b\left(\frac{1}{4} d_{s}^{2} d_{f}+\frac{1}{2} d_{s} d_{f}^{2}+\frac{1}{3} d_{f}^{3}\right)} \approx 4 \frac{k_{f}}{b d_{s}^{2} d_{f}}=4 \frac{\left(k_{s+f}-k_{s}\right)}{b d_{s}^{2} d_{f}}
$$

da die Dicke des Films sehr viel kleiner ist als die des Substrats. Dieser E-Modul des Films bestimmt sich aus der Differenz der Biegesteifigkeit des Systems Substrat+Film und des Substrats. Da diejenige des Substrats sehr viel größer ist als die des Films, ist die Messung stark fehlerbehaftet. Die Erfassung der Frequenz ist sehr genau, womit sich die Messung nur verbessern lässt, indem der Anteil des Substrats reduziert wird. Dies würde jedoch die Qualität der Messung der mechanischen Dämpfung verschlechtern, für welche diese Methode eigentlich ausgelegt ist.

Zuletzt soll nun der Verlustmodul des Films bestimmt werden. Die Güte $Q^{-1}$ ist definiert als der Energieverlust pro Schwingung relativ zur Gesamtenergie

$$
Q^{-1}=\frac{\Delta W}{W 2 \pi}\left(=\frac{2 \Lambda}{\omega}\right)
$$

Dabei ist die gespeicherte Energie $W$ das Produkt aus der Biegesteifigkeit $k$, der Länge $l$ und der Krümmung $\kappa$. Damit gilt $\Delta W=2 \pi Q^{-1} W=2 \pi Q^{-1} k l \kappa$. Außerdem gilt für das System aus Substrat und Film

$$
Q_{s+f}^{-1}=\frac{1}{2 \pi} \frac{\Delta W_{s}+\Delta W_{f}}{W_{s}+W_{f}} \approx \frac{1}{2 \pi} \frac{\Delta W_{s}+\Delta W_{f}}{W_{s}}
$$

da $W_{s} \gg W_{f}$. Setzt man nun die Werte ein und löst nach $Q_{f}^{-1}$ auf so erhält man für den Gütefaktor der Schicht

$$
Q_{f}^{-1}=\frac{1}{3} \frac{d_{s}}{d_{f}} \frac{E_{s}^{\prime}}{E_{f}^{\prime}}\left(Q_{s+f}^{-1}-Q_{s}^{-1}\right)
$$

Da weiterhin gilt

$$
Q_{f}^{-1}=\tan \Delta=\frac{E_{f}^{\prime \prime}}{E_{f}^{\prime}}
$$

mit der Phasenverschiebung $\Delta$, folgt der Verlustmodul und Imaginärteil des komplexen E-Moduls

$$
E_{f}^{\prime \prime}=Q_{f}^{-1} E_{f}^{\prime}=\frac{1}{3} \frac{d_{s}}{d_{f}} E_{s}^{\prime}\left(Q_{s+f}^{-1}-Q_{s}^{-1}\right) .
$$


Diese Messung ist sehr viel genauer als die des Realteils. Die Auflösung wird durch die Dämpfung des Substrats begrenzt, die sich wenn notwendig auch noch weiter reduzieren lässt. 


\section{Gepulste Laserdeposition von PMMA}

Aufgrund der höheren Komplexizität der zu übertragenden Moleküle ist der Prozess der gepulsten Laserdeposition (PLD) von Polymeren vielschichtiger als der anderer Materialien. Geht man davon aus, dass die Polymere wie beispielsweise Metalle vollständig bis auf die atomare Ebene zerlegt würden, wäre es ausgeschlossen, dass diese auf dem Substrat einen Film des Ausgangsmaterials erzeugen. Auch die Temperaturen von über $10.000 \mathrm{~K}$, die für das Ablationsplasma von Metallen gemessen wurden, zerstören organisches Material vollständig. So lassen sich mit KrF Excimer-Lasern einige Polymere, z. B. Polyimid [116] oder Polystyrol, leicht abtragen, die deponierten Filme zeigen jedoch keine charakteristischen Linien im Infrarotsprektrum (Abb. 4.1). Dies bedeutet, dass die polymeren

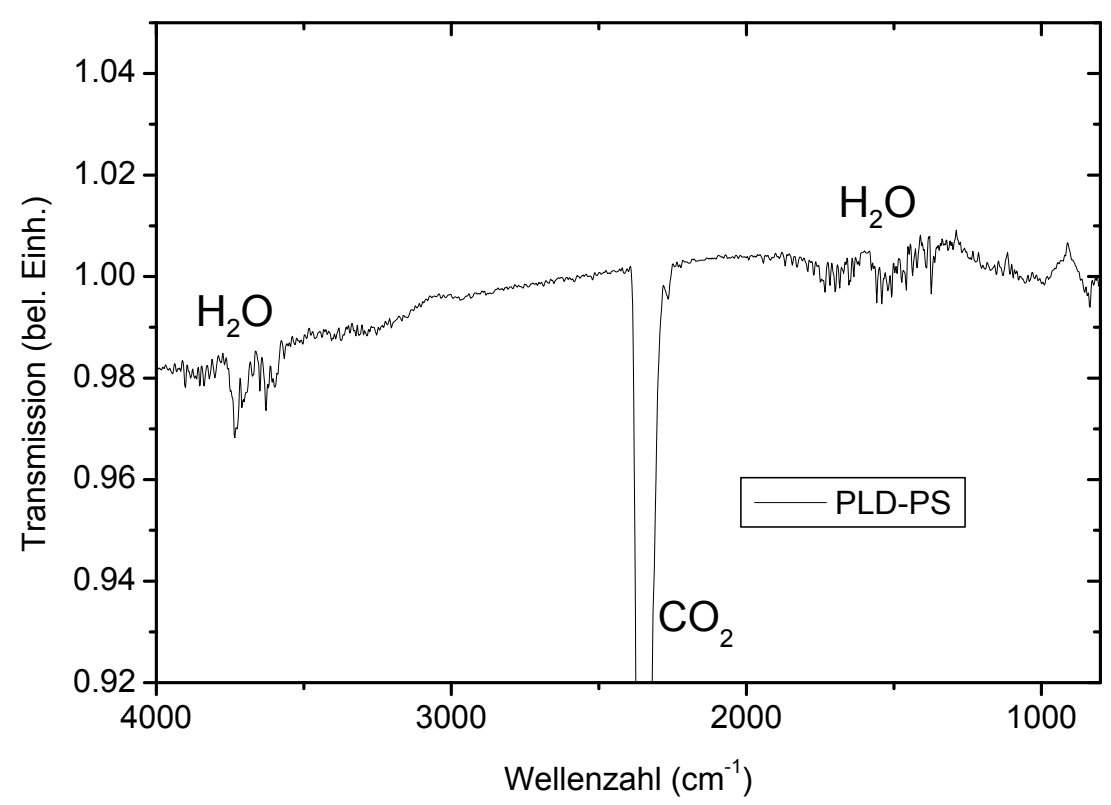

Abbildung 4.1: IR-Spektrum eines von einem Polystyrol-Target laserdeponierten Films

Bindungen zerstört und damit der polymere Charakter verloren ist. Für andere Polymere wie z. B. Poly-bisphenol-A-Carbonat existiert ein kleiner Bereich in der Laserfluenz, direkt oberhalb der Ablationsschwelle, in dem polymere Strukturen übertragen werden [36, 35]. 
Auch wenn für diese Filme die genaue Charakterisierung noch aussteht, steht fest, dass das Material deutlich oberhalb der Ablationsschwelle praktisch völlig zerstört wird.

Vollkommen anders ist die Situation bei Poly(methyl methacrylat) (PMMA, Strukturformel siehe Abb. 4.2). Dieses Material ist für UV-Licht weitgehend transparent, mit einer
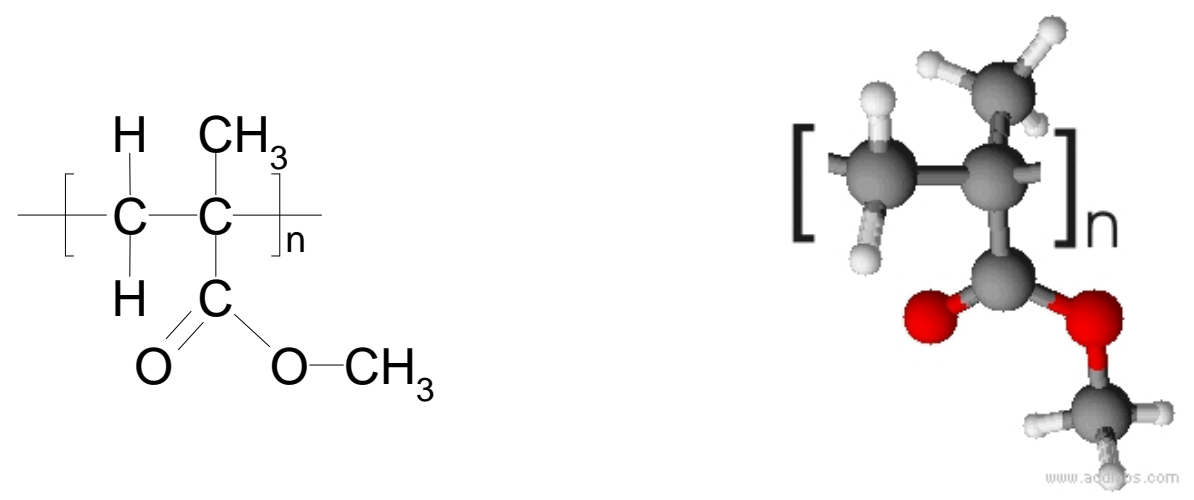

Abbildung 4.2: Strukturformel von Poly(methyl methacrylat) (PMMA)

Eindringtiefe von ca. $150 \mu \mathrm{m}$ für reines PMMA [70]. Dadurch ist die absorbierte Energie pro Volumen zu gering für einen direkten thermischen Abtrag. Allerdings reicht die Photonenenergie von KrF Excimer-Lasern mit $5 \mathrm{eV}$ aus, um die meisten kovalenten Einfachbindungen zu brechen (3-3,6 eV für die $\mathrm{C}-\mathrm{C}$ Bindungen [76]). Nur Doppelbindungen haben eine höhere Energie als $5 \mathrm{eV}(\sim 7,3 \mathrm{eV}$ für $\mathrm{C}=\mathrm{O}$ [81]). Andererseits koppelt das UV-Licht praktisch nicht in die Einfachbindungen ein, Chromophore sind in diesem Fall im Wesentlichen die Carbonylbindungen [88. Der Anregungsmechanismus ist vor allem eine Anregung vom Grundzustand $S_{0}$ in einen angeregten Singulett-Zustand $S_{1}$. Direkte Anregungen in höhere Zustände für Einzelphotonenprozesse sowie Multiphotonenprozesse sind zu vernachlässigen [86]. Der angeregte Zustand kann entweder direkt oder über einen Übergang in einen Triplett-Zustand $T_{1}$ zur Dissoziation führen. Diese beiden Möglichkeiten würde man als photochemische Ablation bezeichnen [108. Wird die Energie zunächst über weitere intramolekulare Prozesse an die nächstliegenden Bindungen weiterverteilt, oder über intermolekulare Mechanismen in Form von Wärme auch auf umliegende Ketten, spräche man von photothermischen Mechanismen. Hierdurch kann Energie über ein Volumen, das sehr groß gegenüber einer einzelnen Bindung ist, eine Temperatur erreichen, die ausreicht um das Polymer zu verändern.

Welcher dieser beiden Mechanismen vorherrscht, wird in der Literatur seit langem kontrovers diskutiert [112, 65, 3], es scheint sich jedoch zumindest für die Bestrahlung bei $248 \mathrm{~nm}$ um einen zweistufigen Prozess zu handeln, der beide Teile nacheinander beinhaltet 
und im folgenden beschrieben werden soll. (Aktuelle Reviews zu diesem Thema finden sich von Dyer [27] oder Chrisey [19].)

\subsection{Inkubation}

Der erste Prozess ist die so genannte Inkubation [121. Hierbei wird das Polymer durch die eintreffenden Photonen stark verändert, ohne dass nennenswerte Ablation auftritt. Beobachtet wurde das Phänomen hauptsächlich bei der Bestrahlung mit Laserfluenzen deutlich unterhalb der Ablationsschwelle. Zum einen beobachtet man teilweise eine Verfärbung der bestrahlten Oberfläche von farblos transparent nach hellgelb, teilweise gelblich braun [74] (siehe Abb. 4.3). Gleichzeitig wird die chemische Struktur des Targets durch die Bestrah-

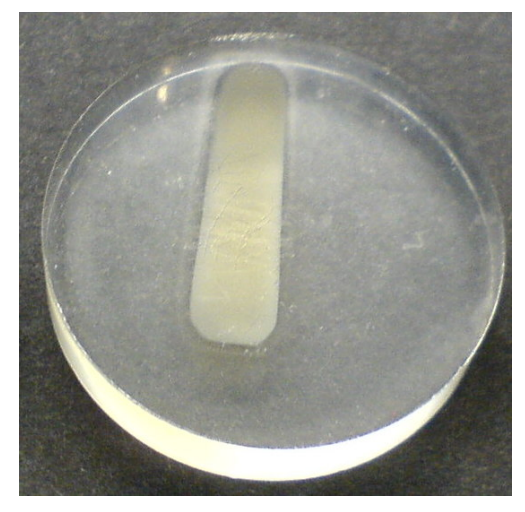

Abbildung 4.3: Laserbestrahltes Target bei $248 \mathrm{~nm}$ mit 40.000 Pulsen bei $20 \mathrm{~mJ} / \mathrm{cm}^{2}$ [74]

lung mit 248 nm Wellenlänge deutlich verändert. Durch die Bestrahlung mit 900 Pulsen bei $39 \mathrm{~mJ} / \mathrm{cm}^{2}$ wird die Kettenlänge des Polymers von $500 \mathrm{~kg} / \mathrm{mol}$ auf $8 \mathrm{~kg} / \mathrm{mol}$ reduziert [10], die Löslichkeit in Lösungsmitteln wird durch die Bestrahlung deutlich erhöht [109]. Die Transmission des UV-Lichts bei $248 \mathrm{~nm}$ sinkt durch 1000 Pulse bei $40 \mathrm{~mJ} / \mathrm{cm}^{2}$ auf 6\% des Ausgangswertes [67].

Für diese sehr niedrigen Fluenzen tritt höchstens eine langwellige Aufrauung der Oberfläche auf, jedoch praktisch kein messbarer Abtrag. Erst nach einigen 10 bis zu tausend Pulsen, abhängig von der Laserfluenz, lässt sich dann Ablation nennenswert beobachten [10. Damit ist die Ablationsschwelle von PMMA bei weitem nicht so klar definiert, wie für andere Materialien. Die chemische Veränderung, die diesem Prozess unterliegt, wurde von Küper und Stuke [67] mittels Infrarotspektroskopie charakterisiert und zum einen mit einer Veränderung der Ester-Seitengruppe, zum anderen mit einer Bildung von $\mathrm{C}=\mathrm{C}-\mathrm{H}$ 
Doppelbindungen in der Hauptkette beschrieben. Dies deutet, konsistent mit den Ergebnissen der chromatographischen Untersuchungen [10], auf eine massive Veränderung des Materials verbunden mit Kettenlängenverkürzung hin. Das Prozessschema in Abbildung 4.4 fasst die wichtigsten ablaufenden Prozesse zusammen [72].

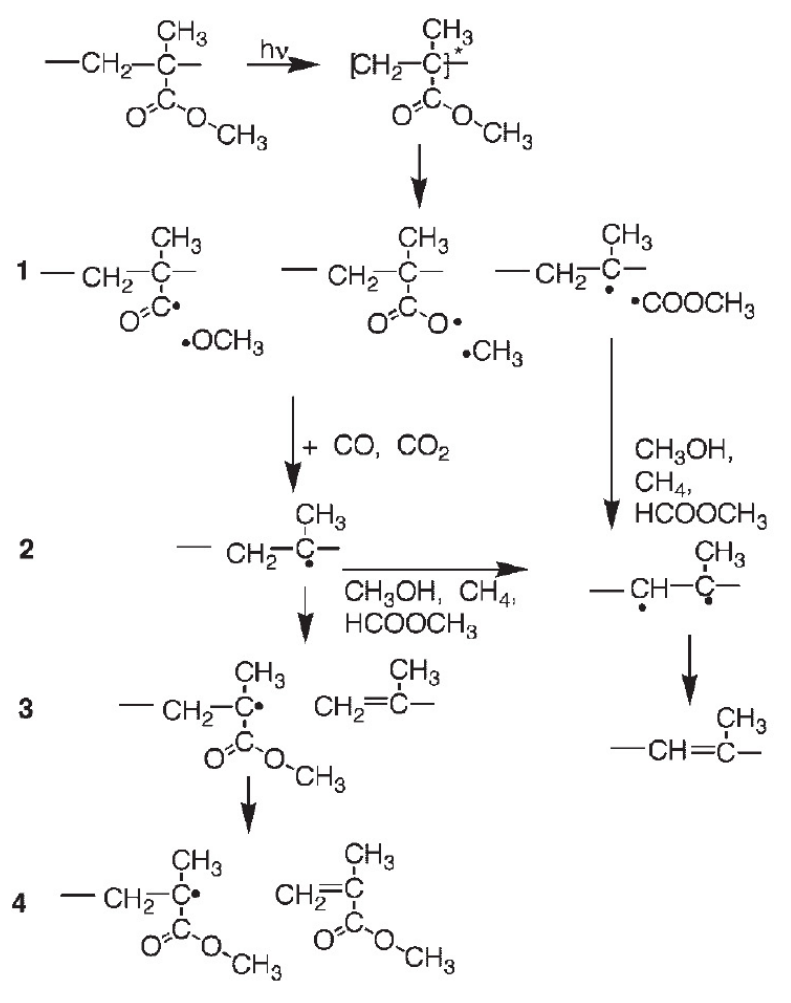

Abbildung 4.4: Prozesse zur Photodissoziation von PMMA nach Lippert [72]

Da dieselben Ergebnisse auch durch die Bestrahlung mit einer UV-Lampe mit vergleichsweise niedriger eingestrahlter Leistung erreicht wurden [67], ist ein photothermischer Prozess sicherlich auszuschließen, die Inkubation erfolgt rein photochemisch. Eine weitere Bestätigung dieser Hypothese ergibt sich dadurch, dass bei einer Wellenlänge von $308 \mathrm{~nm}$ (4,0 eV, XeCl Excimer-Laser) keine Inkubation und überhaupt kein Abtrag beobachtet wurde. Zuvor mit einer Wellenlänge von $248 \mathrm{~nm}$ bestrahltes Material, in dem die Inkubation bereits erfolgt ist, lässt sich dagegen mit $308 \mathrm{~nm}$ Laserpulsen abtragen [67]. Auch dies deutet auch auf einen photochemischen Mechanismus mit mindestens einem Übergang hin, da eine Energie von 4,0 eV ohne Verluste knapp ausreichen würde, um Bindungen aufzubrechen.

Die Inkubationsphase verändert also das Polymer photochemisch zu kürzeren Kettenlängen und sorgt durch die höhere Dichte an Chromophoren (hier Doppelbindungen 
in der Hauptkette) für eine erhöhte Absorption. Dadurch steigt die absorbierte Energie in der Nähe der Oberfläche und die Ablation wird hier vereinfacht.

Nutzt man hohe Laserfluenzen, wie im Rahmen dieser Arbeit geschehen, so ist der Bereich der Inkubation nicht klar zu trennen. Bei Pulsenergien von mehr als einem $\mathrm{J} / \mathrm{cm}^{2}$ reichen wenige Pulse aus, um die erwähnten Effekte zu erreichen. Geht man nur von der eingestrahlten Energie aus, so ist nach etwa 30 Pulsen ein vergleichbares Ergebnis erreicht. Dies wurde untersucht, indem der Abtrag während der ersten Pulse betrachtet wurde. Hierfür wurden 100 Laserpulse bei $1,2 \mathrm{~J} / \mathrm{cm}^{2}$ in abbildender Geometrie auf ein frisches PMMA Target gegeben und mithilfe einer Kamera aufgenommen. Um diese darstellen zu können, wurde die Leuchterscheinung des auftreffenden Laserstrahls jeweils ausgeschnitten und die Bilder in Abbildung 4.5 (oben) nebeneinander gesetzt. Dabei wurde für jeden Puls der gleiche Bereich des Bildes ausgewählt. In dieser Darstellung sieht man, dass die Leucht-

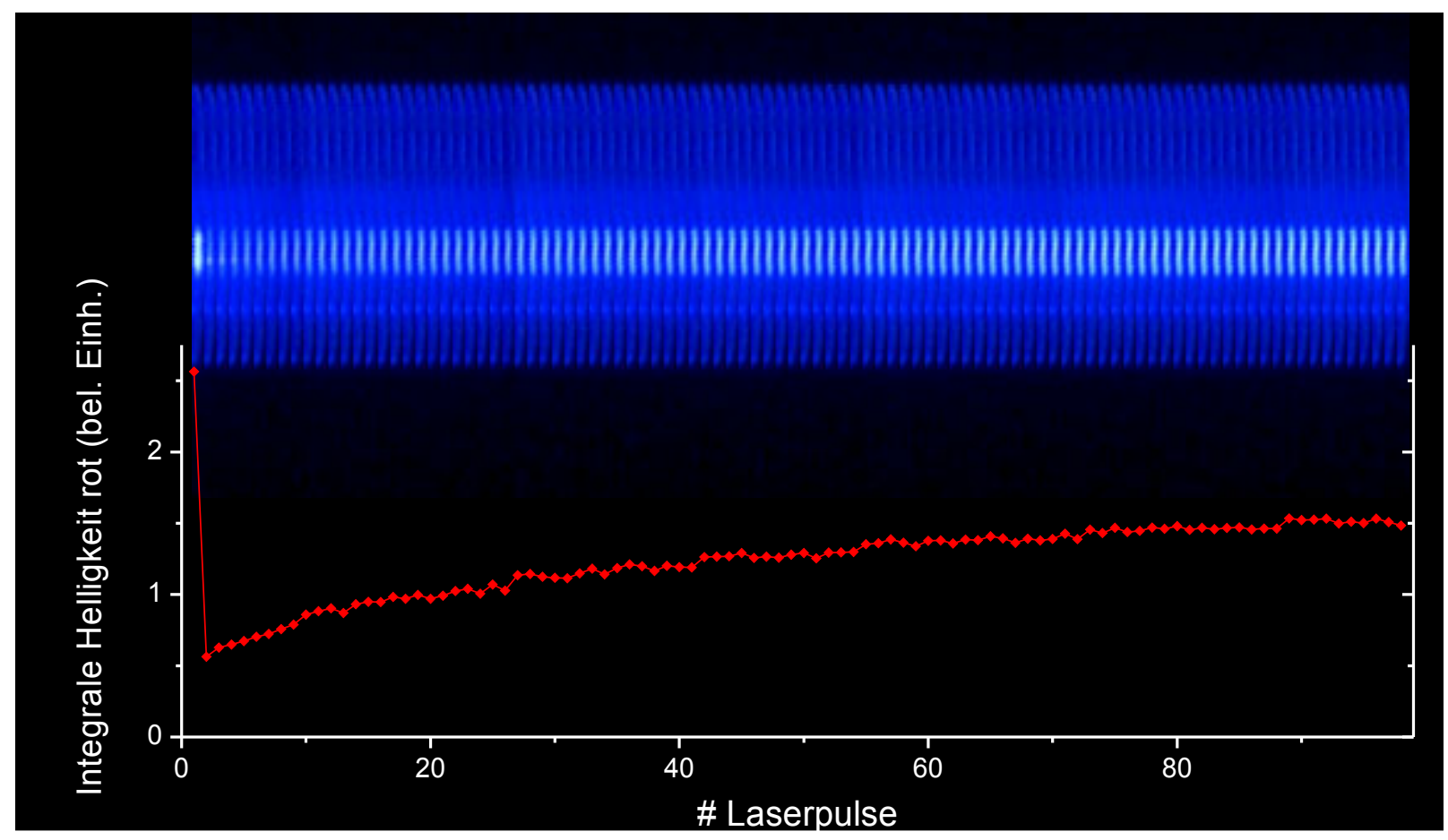

Abbildung 4.5: Oben: Serie von Bildern der ersten 100 Laserpulse auf ein PMMA Target, von links nach rechts angeordnet; Unten: Integral über die Helligkeit der Farbe Rot für jeden Bildausschnitt

erscheinung beim ersten Puls (ganz links) besonders stark ist. Dies ist darauf zurück zu führen, dass die Oberfläche durch die Herstellung der Targets nicht perfekt sauber ist und sich Adsorbate auf der Oberfläche befinden (vgl. [78] für $\mathrm{MgO}$ ). Diese lösen sich durch nur einen Puls ab und das Leuchten wird sehr schwach. Während der ersten 70 Pulse nimmt die 
Intensität kontinuierlich zu und erreicht danach einen gleichmäßigen Wert. Dieser Eindruck kann bestätigt werden, indem durch ein selbstgeschriebenes Programm über die Helligkeit jedes Einzelbildausschnitts integriert wird. Da die Helligkeit des Farbkanals blau im zentralen Bereich in Sättigung geht, wurde für das Integral nur der Rotkanal ausgewählt. Die Kurve wird im unteren Teil der Abbildung 4.5 gezeigt und bestätigt die qualitative Aussage. Im Vergleich mit Messungen für $193 \mathrm{~nm}(6,4 \mathrm{eV})$ ist die für die Beendigung der Inkubationsphase benötigte Pulszahl sehr hoch, Srinivasan et al. [109] beobachten 20-40 benötigte Pulse bei nur $0,16 \mathrm{~J} / \mathrm{cm}^{2}$, was wieder die These des photochemischen Mechanismus unterstützt.

Diese Messung wird durch die Untersuchung der Depositionsrate während der ersten Pulse weiter bestätigt. Sie erfolgt über eine Schwingquarzwaage direkt gegenüber dem Target an der Stelle des Substrats. Die Messung ist in Abbildung 4.6 gezeigt. Es ist zu

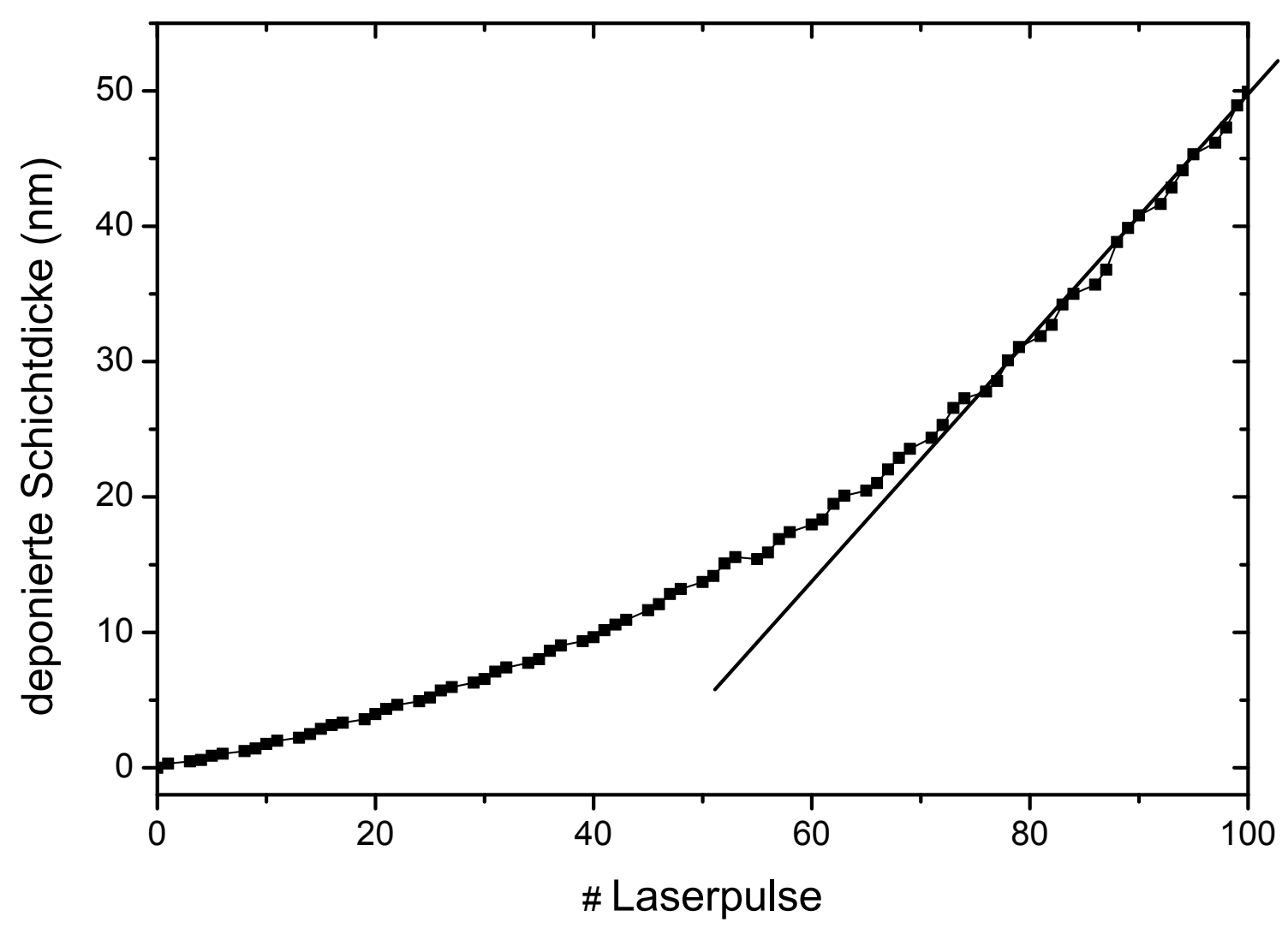

Abbildung 4.6: Deponierte Schichtdicke von PMMA während der ersten 100 Laserpulse bei $1,2 \mathrm{~J} / \mathrm{cm}^{2}$ 
sehen, dass während der ersten ca. 70 Pulse eine deutlich reduzierte Rate gemessen wird. Danach steigt sie auf einen üblichen Wert von $1 \mathrm{~nm} /$ Puls an.

Die beobachtete Ablation und Deposition während der ersten Pulse lässt sich durch eine Überlagerung der verschiedenen Prozesse erklären [66]. Die Energie, die tiefere Schichten erreicht, fällt nach dem Lambert-Beerschen Gesetz exponentiell ab. Damit werden die obersten Schichten am stärksten verändert und schon während des ersten Pulses abgetragen. Während der weiteren Pulse tritt eine starke Veränderung in Lagen bis zu $10 \mu \mathrm{m}$ Tiefe auf [74]. Durch die gestiegene Absorption kann das Laserlicht keine noch tieferen Stellen mehr erreichen und es tritt ein Gleichgewicht zwischen Inkubation, Abtrag und erneuter Inkubation ein. Da schon ab dem ersten Puls Energie aufgewendet wird, um Material abzutragen, werden für die Inkubation ins Gleichgewicht nicht 30 Pulse benötigt, wie vorher abgeschätzt, sondern ca. 70 .

\subsection{Laser-Ablation von PMMA}

Für die Ablation können analog zu den Metallen im Weiteren zwei Ablationsprodukte unterschieden werden. Zum einen niedermolekulares Material, das weitgehend gasförmig ist und sich im Film nicht wieder findet, zum anderen Tröpfchen aus polymerem Material.

\subsubsection{Kleine Fragmente}

Kleine Fragmente lassen sich während der Deposition über Massenspektroskopie nachweisen. Zu diesem Zweck wurde ein Quadropol Massenspektrometer mit einem Nachweisbereich bis zu $300 \mathrm{amu}$ benutzt. Da eine Beschichtung des Spektrometers weitmöglich vermieden werden sollte, befindet es sich während der Messung im abgeschatteten Bereich hinter dem Target. Dies ergibt die Einschränkung, dass der gemessene Druck sehr viel kleiner ist als in der Plasmakeule und große Bestandteile, die nichtflüchtig deponiert werden, überhaupt nicht nachgewiesen werden können.

Die Daten, die in Zusammenarbeit mit Süske [117] entstanden sind, sind in Abbildung 4.7 gezeigt und mit denen der Thermolyse von PMMA bei $300{ }^{\circ} \mathrm{C}$ verglichen. Dies ist notwendig, da Quadropol-Massenspekrometer durch die unsanfte Ionisation die organischen Bruchstücke weiter zerlegen. Für die thermische Degradation von PMMA ist bekannt, dass hauptsächlich Monomere entstehen [55]. Durch den Vergleich ist es möglich den Anteil an Monomeren aus den hier erfolgten Messungen abzuleiten und damit den photothermischen Anteil an der Ablation abzuschätzen. Im oberen Bild von Abbildung 4.7 sind die Daten 


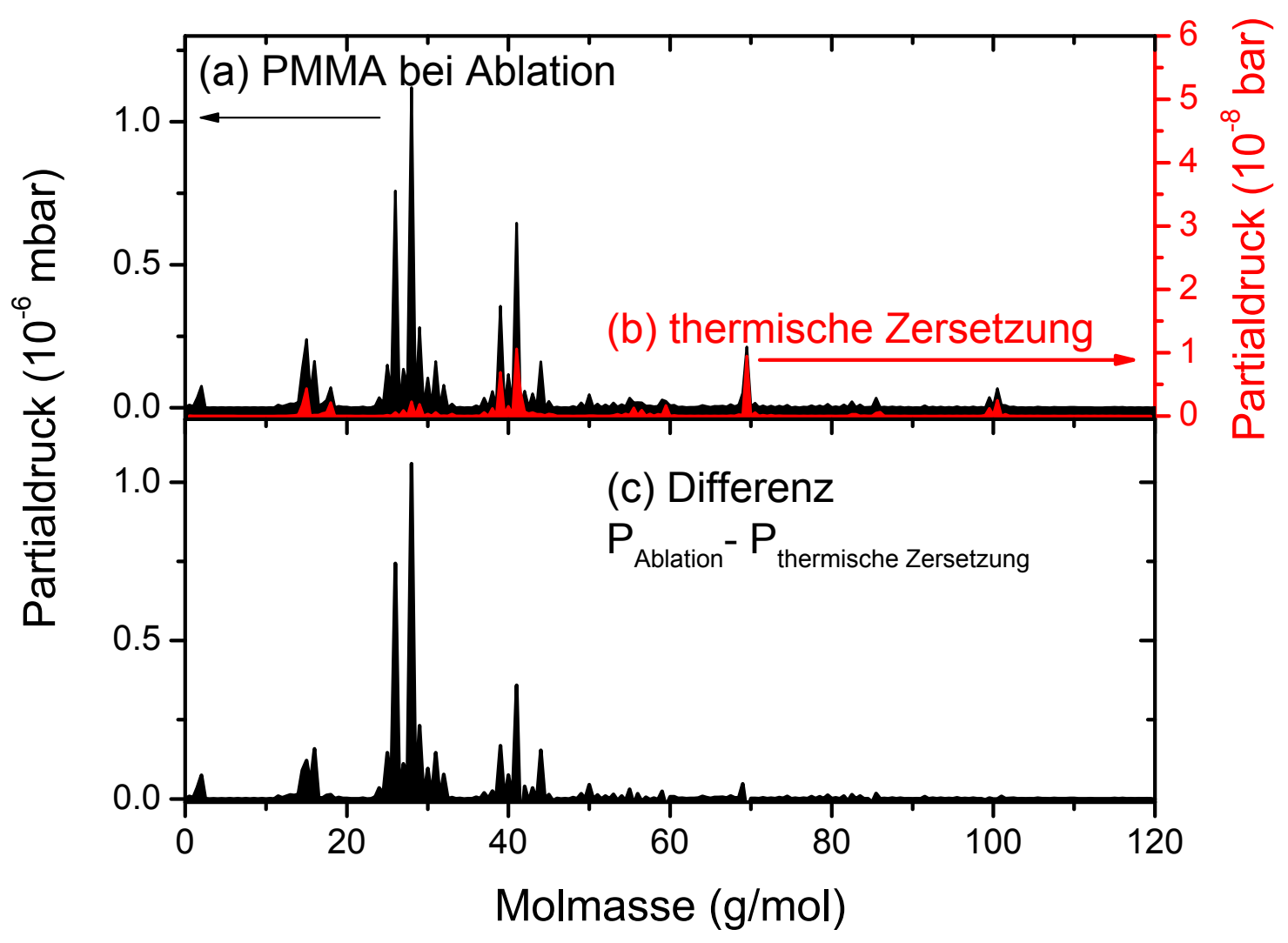

Abbildung 4.7: (a) Massenspektrum während der Laserdeposition von PMMA; (b) Massenspektrum für thermisches Zersetzen von PMMA; (c) Differenz von (a) und (b), normiert auf den Partialdruck bei $100 \mathrm{~g} / \mathrm{mol}$; aus [117]

dieser beiden Prozesse dargestellt. In dem unteren Teil der Abbildung ist die Differenz dieser beiden Kurven aufgetragen. Dazu wurde die der Thermolyse für die Monomermasse von $100 \mathrm{~g} / \mathrm{mol}$ auf diejenige der Laserdeposition normiert und davon abgezogen. Geht man nun davon aus, dass bei der Thermolyse nur Monomere entstanden sind, kann man damit auf den Anteil an Monomeren zurück schließen und kommt auf ca. 40 Gew.\%. Der Rest im Differenzspektrum sind kleinere Bruchstücke, die hauptsächlich durch ein Abspalten und Zerlegen der Estergruppe erklärt werden können [117]. Oberhalb von $120 \mathrm{~g} / \mathrm{mol}$ sind keine weiteren Peaks zu erkennen, im besonderen keine Di- und Trimere.

Das Differenzspektrum zwischen thermischem Zersetzen und Laserdeposition zeigt deutlich, dass es Unterschiede gibt, dass nicht nur photothermisch zersetzte Anteile auftreten. Vermutlich resultieren die kleinen Anteile hauptsächlich aus photochemischen Prozessen, vor allem da sie aus der Estergruppe stammen [117], in die das Laserlicht einkoppelt. Aufgrund von Flugzeitspektren schließt Srinivasan [111] aus, dass die kleinen Anteile pho- 
tothermisch entstanden sein können, da die Lebensdauer der photothermischen Zustände [55] um Größenordnungen zu hoch ist. Die hier betrachteten Anteile kleiner Moleküle sind erheblich höher, als sie teilweise in der Literatur dargestellt werden [7] (für $266 \mathrm{~nm}$ Wellenlänge). Es ist allerdings zu bemerken, dass die Anteile je nach Wellenlänge [32] oder Laserfluenz deutlich unterschiedlich sein können. So teilt Srinivasan [111] drei Fluenzbereiche ein, im ersten werden hauptsächlich kleine Bruchstücke erzeugt, oberhalb einer Schwelle effizienter Ablation werden auch größere Bruchstücke abgetragen und die Abtragstiefe nimmt stark zu. Im Bereich drei, der knapp oberhalb der hier untersuchten Laserenergie beginnt, wird dann die Abtragstiefe nicht weiter gesteigert und die Effizienz nimmt deutlich ab. Bei hohen Fluenzen steigt das Verhältnis von photothermischem zu photochemischem Anteil an [113].

\subsubsection{Droplets}

Weitere Produkte der Ablation sind Tröpfchen bestehend aus Polymer verkürzter Kettenlänge. Diese wurden schon von Srinivasan [110] durch die Aufnahmen mit einer hochauflösenden Hochgeschwindigkeitskamera beobachtet, und nachgewiesen, dass sie erst signifikant nach dem Ende des Laserpulses austreten. Damit ist es möglich, dass ihre Herkunft photothermisch ist. Srinivasan beschreibt, dass sie als lange, nadelförmige Kristalle aus dem Target kommen und erst während des Fluges aufschmelzen und eine runde Form annehmen [108].

In der Folge der Arbeit von Süske [117] und den Ergebnissen dieser Arbeit bietet sich eine andere Erklärung der beobachteten Bilder an. Durch das Erhitzen des Materials unter der Oberfläche und die teilweise Zersetzung bilden sich flüssige Blasen, die durch die gasförmigen Produkte der chemischen Veränderung unter hohem Druck stehen. Platzen diese Blasen, so schießt das Innere unter hohem Druck strahlförmig hinaus und bildet erst während des Fluges die durch die Oberflächenspannung gebotene Kugelform.

Diese Annahme wird vor allem durch die Betrachtung der bestrahlten Oberfläche begründet (Abb.4.8). Von der Oberseite aus betrachtet ist sie übersäht mit einer großen Zahl statistisch verteilter Löcher ähnlicher Größe. Betrachtet man das Target im Querschnitt, was durch sprödes Brechen durch den bestrahlten Bereich möglich ist, so sieht man, dass ein Bereich von etwa $10 \mu \mathrm{m}$ Tiefe stark verändert ist. Er besteht aus einer schwammartigen Struktur von leeren Blasen, bei einigen ist eine Öffnung nach oben zu erkennen, aus der das flüssige Material ausgetreten sein dürfte [74]. 

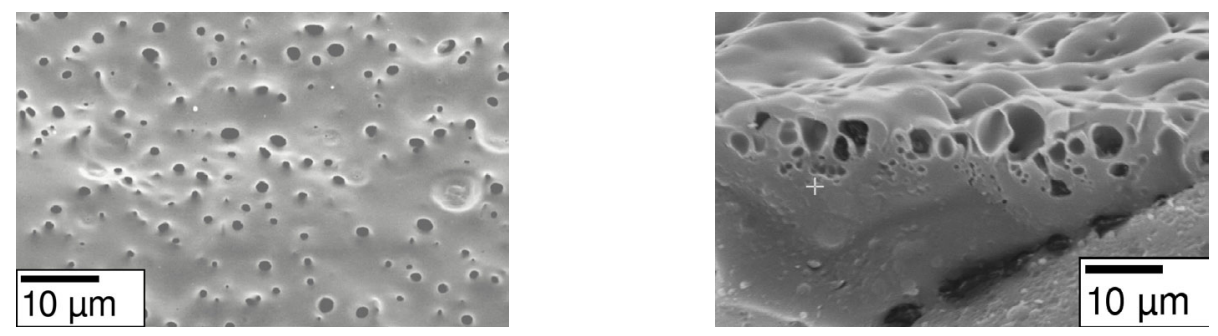

Abbildung 4.8: Rasterelektronenmikroskop Bilder von PMMA Targets, bestrahlt mit 100 Pulsen. Links: Aufsicht; Rechts: Querschnitt [74], jeweils ca. 800-fache Vergrößerung

Efthimiopoulos et al. [31] beobachten für Bestrahlung bei 308 nm Wellenlänge bei niedriger Laserfluenz $\left(2,7 \mathrm{~J} / \mathrm{cm}^{2}\right)$, dass die Blasen über die Dauer einiger Pulse entstehen, wachsen und schließlich aufbrechen. Bei hohen Fluenzen $\left(11 \mathrm{~J} / \mathrm{cm}^{2}\right)$ tritt dies schon bei einem Puls auf, analog zu dem Modell des explosiven Verdampfens [79]. Bei mittleren Fluenzen lässt sich beobachten, dass die Blasen bei ungeraden Pulszahlen entstehen, bei den darauf folgenden, geraden platzen. Durch akustische Messungen wurde nachgewiesen, dass durch das Platzen der Blasen der Hauptmaterialabtrag stattfindet [28]. Die großen Unterschiede in der Laserfluenz im Vergleich zu dieser Arbeit sind in der unterschiedlichen Wellenlänge begründet. Bei mit 308 nm Wellenlänge bestrahltem PMMA bestimmten Tsunekawa et al. [130] mithilfe der Maxwell-Boltzmann-Verteilung von Geschwindigkeiten die Temperatur der Monomere auf $600 \mathrm{~K}$, die anderer, vor allem größerer Bestandteile bis zu $1200 \mathrm{~K}$, was mit etwas Vorsicht einen Rückschluss auf die Temperaturen in den Blasen zulässt.

\subsection{Deposition}

Betrachtet man die Filme, die durch die gepulste Laserdeposition von PMMA entstehen, so spiegelt sich genau die zweikomponentige Struktur aus der Ablation wieder. Dies ist auf den Rasterelektronenmikroskopischen Bildern in Abbildung 4.9 zu sehen. Die Filme bestehen
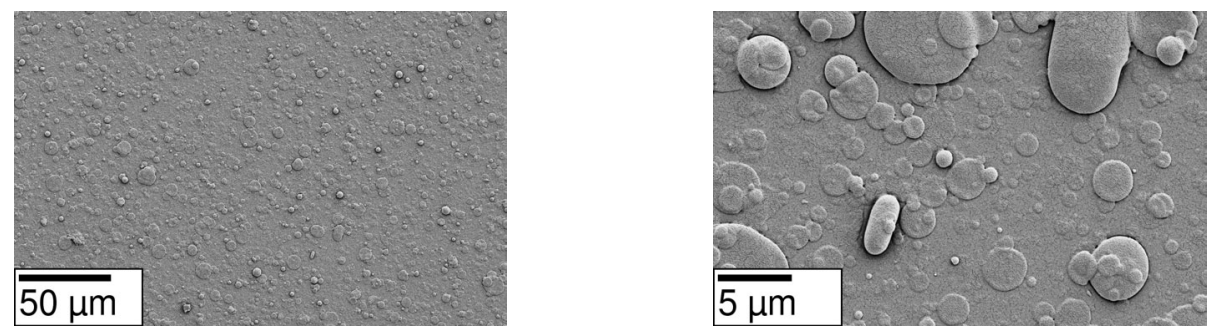

Abbildung 4.9: Rasterelektronenmikroskop Bilder eines PMMA Films, mit 300 Pulsen bei 1,2 J/ $\mathrm{cm}^{2}$. Links: ca. 200-fache Vergrößerung; Rechts: ca. 1.600-fache Vergrößerung 
aus einer glatten Schicht, kombiniert mit einer großen Anzahl von Droplets. Diese sind meist rund, einige $\mu \mathrm{m}$ im Durchmesser, jedoch nur einige hundert Nanometer hoch [117]. Kleinere Droplets nähern sich häufig stärker an die Halbkugelform an und es gibt auch nichtrunde Formen.

\subsubsection{Glatter Filmanteil}

Betrachtet man die Bereiche zwischen den Droplets, so ist festzustellen, dass sich auch hier ein Film befindet, der im Weiteren als glatte Komponente bezeichnet werden soll. Diese ist unlöslich in den üblichen organischen Lösungsmitteln, was darauf schließen lässt, dass sie aus hochvernetztem Material besteht. Von Süske [117] wurde durch Lösen der sonstigen Komponenten gezeigt, dass dieser Anteil immer noch weite Anteile des IR-Spektrums von PMMA zeigt, die Carbonylbindung und die Methylgruppen jedoch stark reduziert sind, so dass von einer Vernetzung über diese ausgegangen werden kann.

Rauigkeit und Dicke dieser Komponente sind durch die Röntgenreflektometrie messbar. In Abbildung 4.10 ist ein solches Spektrum zu sehen. Die Oberflächenrauigkeit (rms), be-

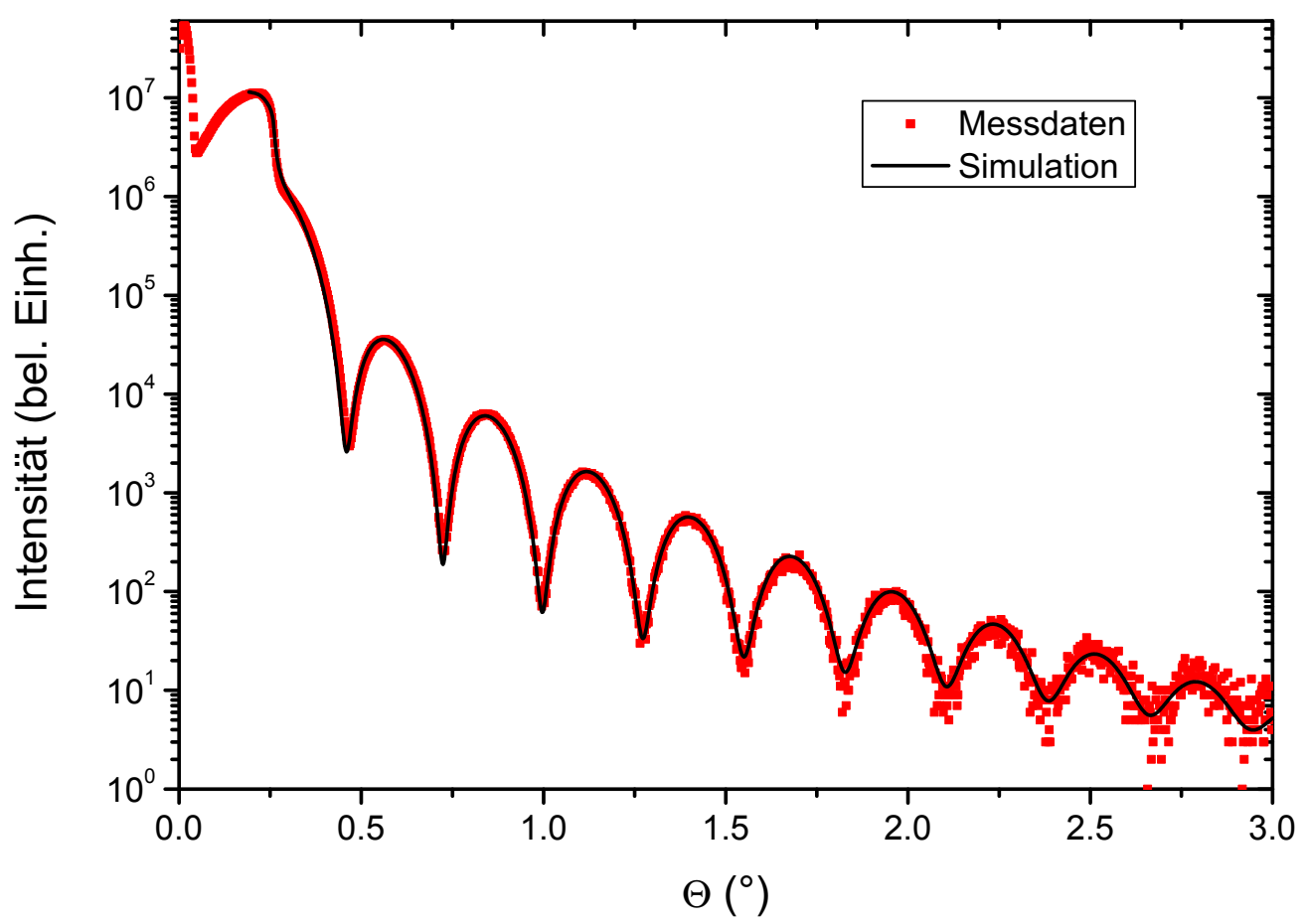

Abbildung 4.10: Röntgenreflektometriemessung an einem mit 500 Pulsen bei 1,2 $\mathrm{J} / \mathrm{cm}^{2}$ deponierten PMMA Film mit zugehöriger simulierter Kurve. Parameter siehe Tabelle 4.1 
stimmt durch die Anpassung einer simulierten Kurve mittels IMD [137], beträgt hier nur 0,53 nm, was fast vergleichbar mit sehr guten Metallfilmen gleicher Dicke ist. Die Simulation passt so gut zu den gemessenen Daten, dass Fehler durch die Anpassung weitgehend auszuschließen sind. Alle Simulationsparameter sind in Tabelle 4.1 zusammengefasst. Der

\begin{tabular}{|c|c|c|c|}
\hline$\rho$ & $d$ & $\sigma_{\text {Substrat }}$ & $\sigma_{\text {Oberfläche }}$ \\
$1,01 \mathrm{~g} / \mathrm{cm}^{3}$ & $18,36 \mathrm{~nm}$ & $0,24 \mathrm{~nm}$ & $0,53 \mathrm{~nm}$ \\
\hline
\end{tabular}

Tabelle 4.1: Anpassungsparameter für die Röntgenreflektometrie aus Abbildung 4.10. bestimmt durch IMD [137]

Wert für die Rauigkeit ist natürlich nur dadurch zu erreichen, dass die Röntgenreflektometrie nur die glatte Komponente betrachtet. Die Droplets selbst zeigen eine deutlich unterschiedliche Höhe und die durch sie erzeugte Rauigkeit wird durch die Kohärenzlänge der Röntgenstrahlung nicht erfasst.

\subsubsection{Droplets}

Die Droplets selbst entstehen aus dem im flüssigen Zustand vom Target abgetragenen Material, was schon aus der Betrachtung der vergleichbaren Größen als sicher gelten kann (vergl. Abb. 4.8 und 4.9). Aus diesem Grund erwartet man zusätzlich zur Inkubation keine weitgehenden Veränderungen der chemischen Zusammensetzung. Ein Infrarotspektrum des gesamten Films ist in Abbildung 4.11 gezeigt. Zum Vergleich ist das Spektrum eines PMMA Targets aufgetragen. Es ist leicht zu sehen, dass im Bereich der experimentellen Unsicherheit praktisch kein Unterschied zwischen diesen Kurven besteht. Das bedeutet, dass die Verhältnisse der chemischen Bindungen im Vergleich zum Target höchstens leicht verändert sind.

Weiterhin ist das Material in den Droplets in organischen Lösungsmitteln wie Tetrahydrofuran (THF) löslich. Dieser lösliche Anteil wurde für die Größenausschlusschromatographie (SEC) verwendet. Die Ergebnisse sind in Abbildung $4.12 \mathrm{zu}$ sehen. Das gelöste Polymer hat eine massenmittlere Molmasse $M_{W}$ von etwa $8 \mathrm{~kg} / \mathrm{mol}$. Dies ist im Vergleich zum Target $(996 \mathrm{~kg} / \mathrm{mol})$ eine deutliche Veränderung. Weiterhin konnte festgestellt werden, dass auch für niedrigere Ausgangsmassen von 350 und $15 \mathrm{~kg} / \mathrm{mol}$ keine weitere Veränderung im Film festzustellen ist. Die Anteile der kleinen Moleküle unter $1.000 \mathrm{~g} / \mathrm{mol}$ lassen sich durch ihre Regelmäßigkeit als verschiedene Oligomere identifizieren, durch die fehlende Kalibrierung der SEC in diesem Bereich ist die Molmassenbestimmung jedoch ungenau. 


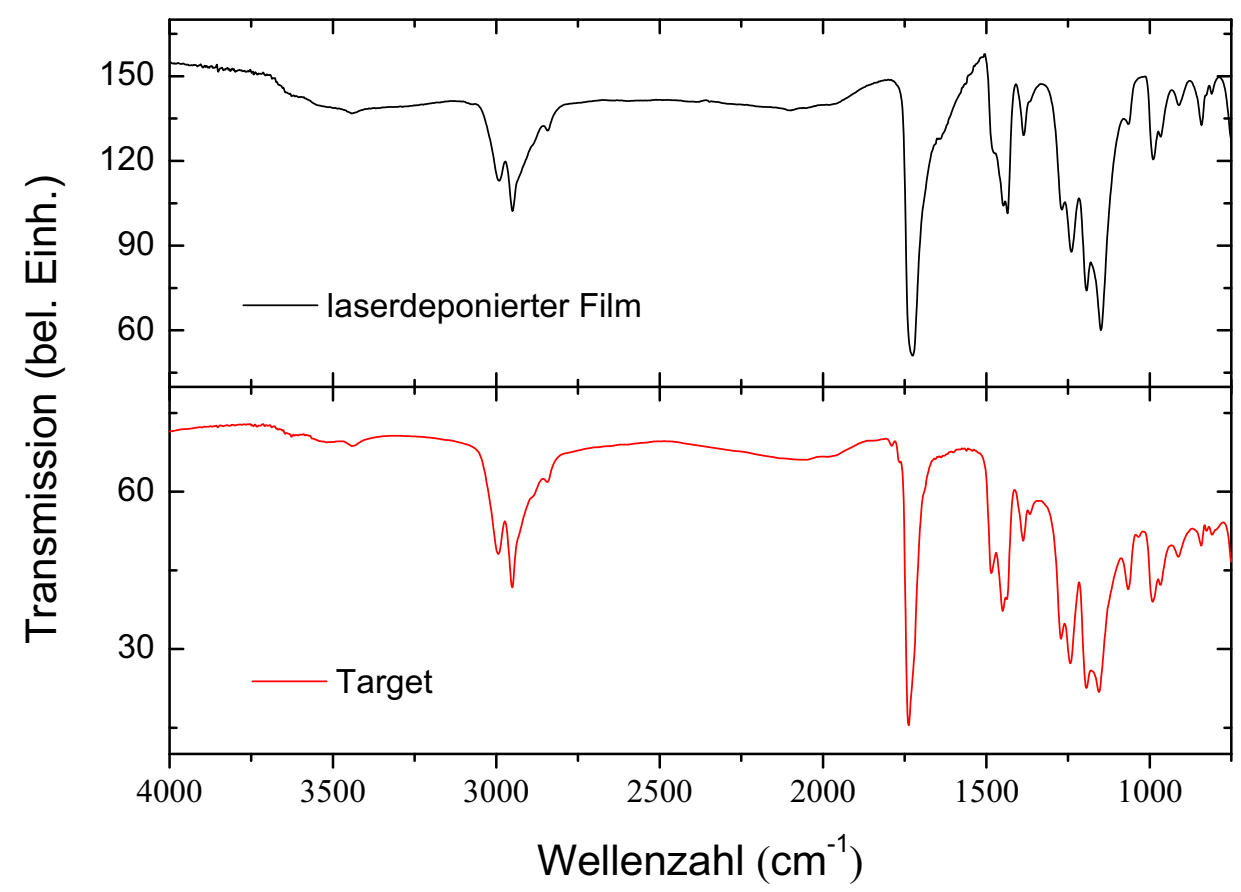

Abbildung 4.11: Infrarotspektren eines laserdeponierten Films und des dazugehörigen Targets

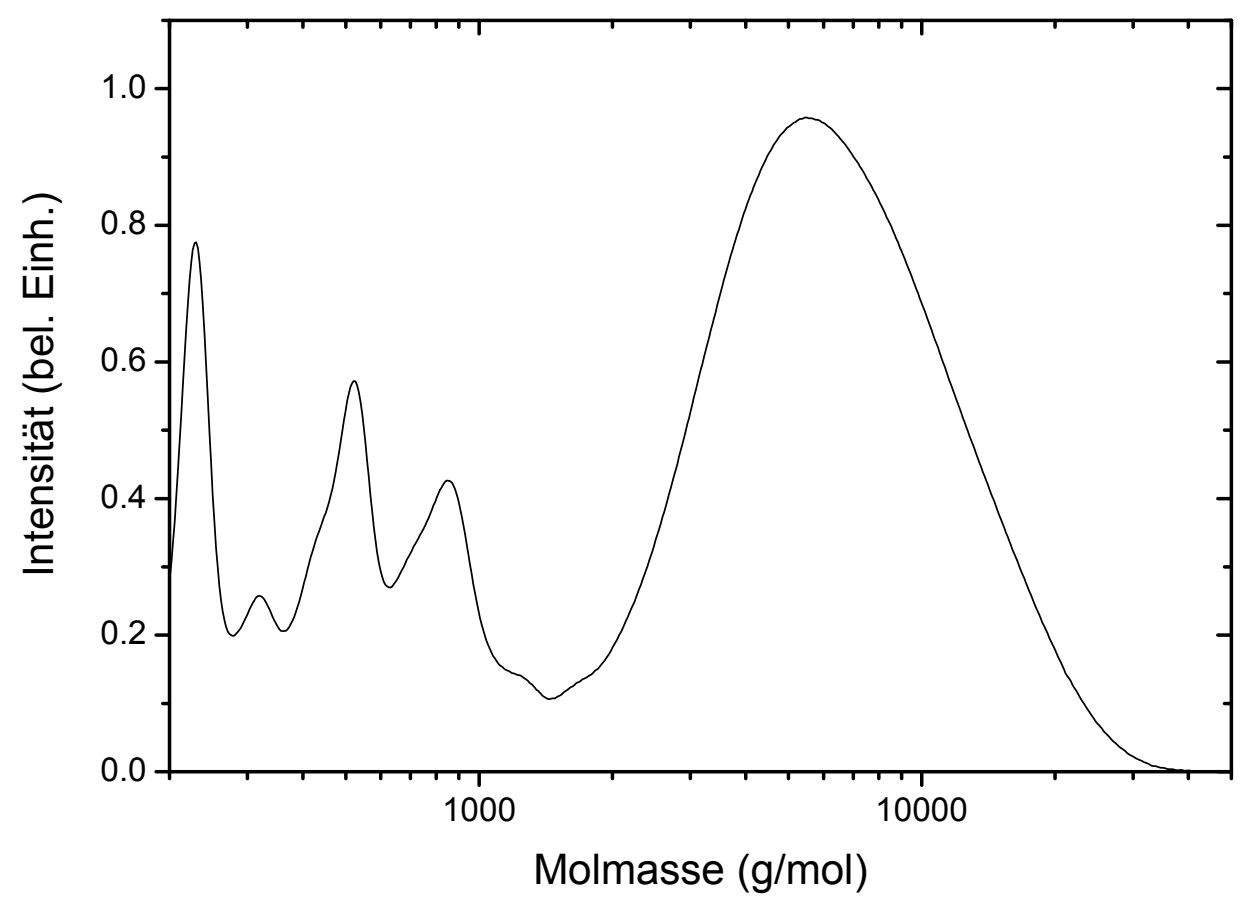

Abbildung 4.12: Chromatogramm eines laserdeponierten PMMA-Films 
Mit der Verwendung höherer Substrattemperaturen während der Herstellung lassen sich dagegen höhere Molmassen im Film erzielen [9]. So steigt $M_{W}$ bei den für diese Arbeit durchgeführten Messungen für eine Substrattemperatur von $250{ }^{\circ} \mathrm{C}$ auf $32 \mathrm{~kg} / \mathrm{mol}$ [118]. Diese Ergebnisse sind in Abbildung 4.13 aufgetragen. Im Inset ist eine Arrhenius-

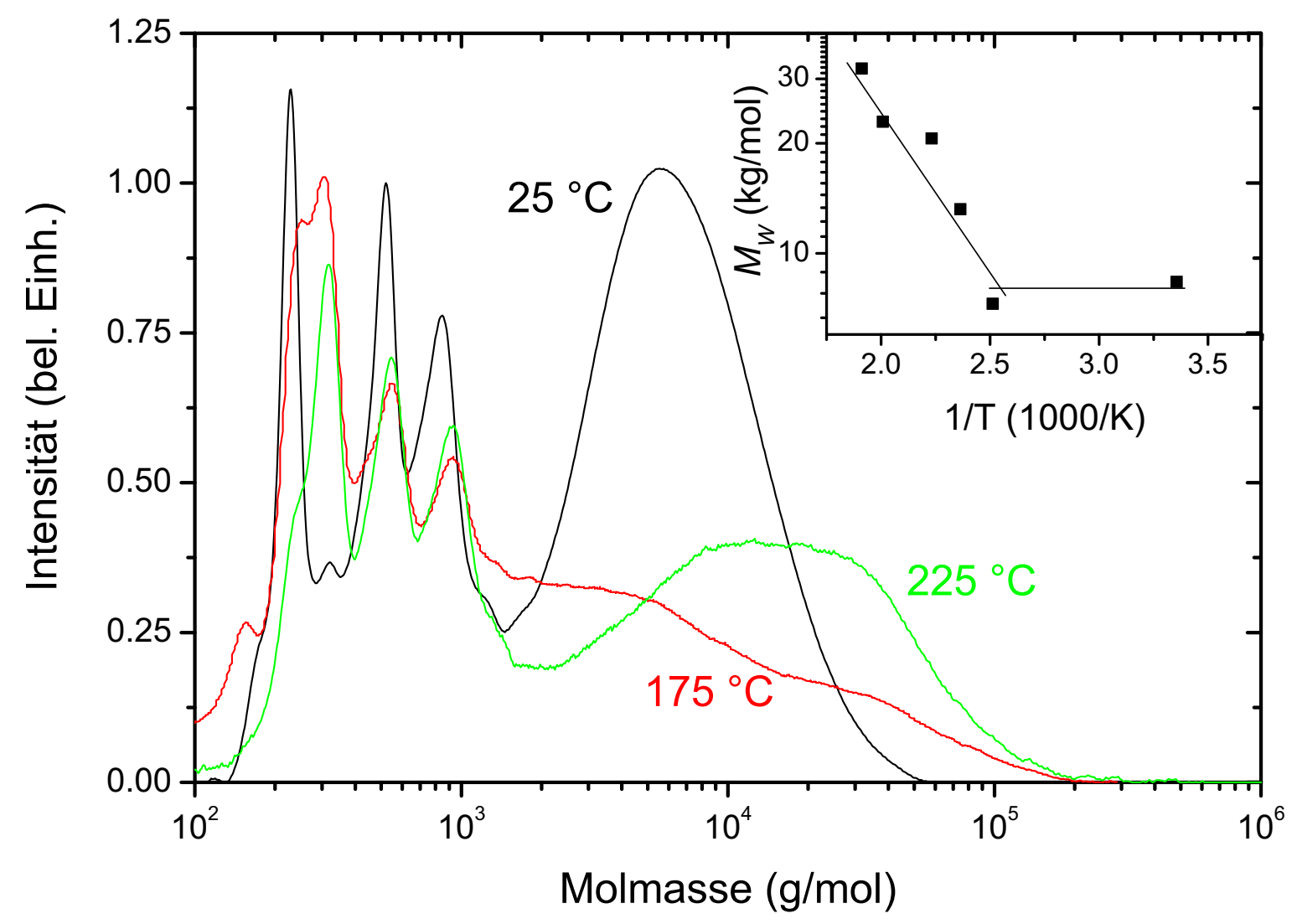

Abbildung 4.13: Chromatogramme für PMMA-Filme, die bei den angegebenen Substrattemperaturen hergestellt wurden. Inset: Molmasse $M_{W}$ für verschiedene Filme in Arrhenius-Auftragung

Auftragung von $M_{W}$ gegen die Temperatur zu sehen. Wie man erkennt, ist für Substrattemperaturen oberhalb von $150{ }^{\circ} \mathrm{C}$ recht gut eine Gerade anzupassen, der Prozess ist durch eine Aktivierungsenergie von $0,2 \mathrm{eV}$ bestimmt.

Damit ergeben sich zwei Möglichkeiten der Interpretation dieser Resultate. Zum einen ist es möglich, dass schon die abgetragenen Droplets eine Molmasse von ca. $8 \mathrm{~kg} / \mathrm{mol}$ aufweisen, zum anderen könnte dies durch Repolymerisation von noch kleineren Bestandteilen erfolgen, vor allem von Monomeren, wie dies in der Literatur teilweise angenommen wird [9] (8] für PTFE). Um dies zu klären, wurde in Zusammenarbeit mit dem Institut für Physika- 
lische Chemie, Universität Göttingen, PMMA mit einer Molmasse von $M_{W} \approx 3.200 \mathrm{~g} / \mathrm{mol}$, also deutlich unterhalb von $8.000 \mathrm{~g} / \mathrm{mol}$, durch kontrollierte radikalische Polymerisation synthetisiert. Die SEC Spuren des Targetmaterials sowie des daraus hergestellten Films sind in Abbildung 4.14 gezeigt. Es ist zu sehen, dass für diese Bereiche der Molmasse Tar-

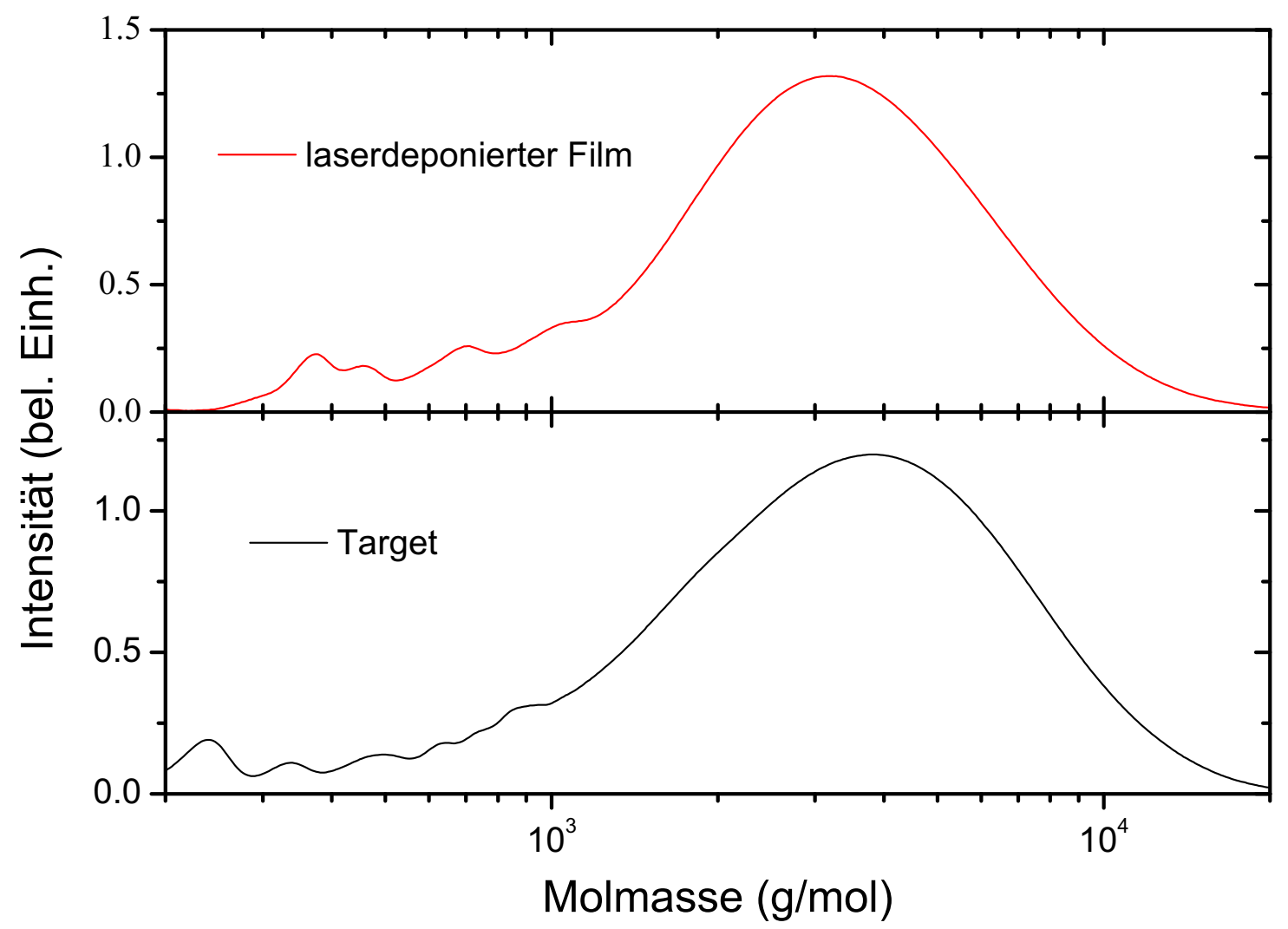

Abbildung 4.14: Chromatogramm eines Targets mit $M_{W} \approx 3.200 \mathrm{~g} / \mathrm{mol}$ und des daraus laserdeponierten Films

gets und Substrate nicht nur über praktisch die gleiche Molmasse verfügen, sondern auch die Form der Kurven fast identisch ist. Weiterhin ist die resultierende Molmasse deutlich unterhalb der sonst üblichen. Damit scheidet der Mechanismus der Repolymerisation von Monomeren am Substrat, wie er von Blanchet et al. 8, 9] vorgeschlagen wurde, aus.

Es bleibt zu klären, ob die Verlängerung der Ketten bei erhöhter Substrattemperatur durch den Zusammenschluss von Makromolekülen erfolgt oder durch Reaktion mit kleineren Einheiten, besonders Monomeren. Dazu wurden PMMA Eichstandards mit $M_{W}=$ $4.200 \mathrm{~g} / \mathrm{mol}$ (PSS) als Targets benutzt. Diese zeichnen sich dadurch aus, dass sie eine sehr scharfe Molmassenverteilung zeigen. Sollte jetzt der Zusammenschluss durch lineare Reaktion der Makromoleküle erfolgen, so sollten sich in den Chromatogrammen wiederum 
scharfe Peaks bei den Vielfachen der einfachen Massen bilden. Ein solches Chromatogramm eines Films, hergestellt bei $190{ }^{\circ} \mathrm{C}$ ist in Abbildung 4.15 aufgetragen. Es ist zu

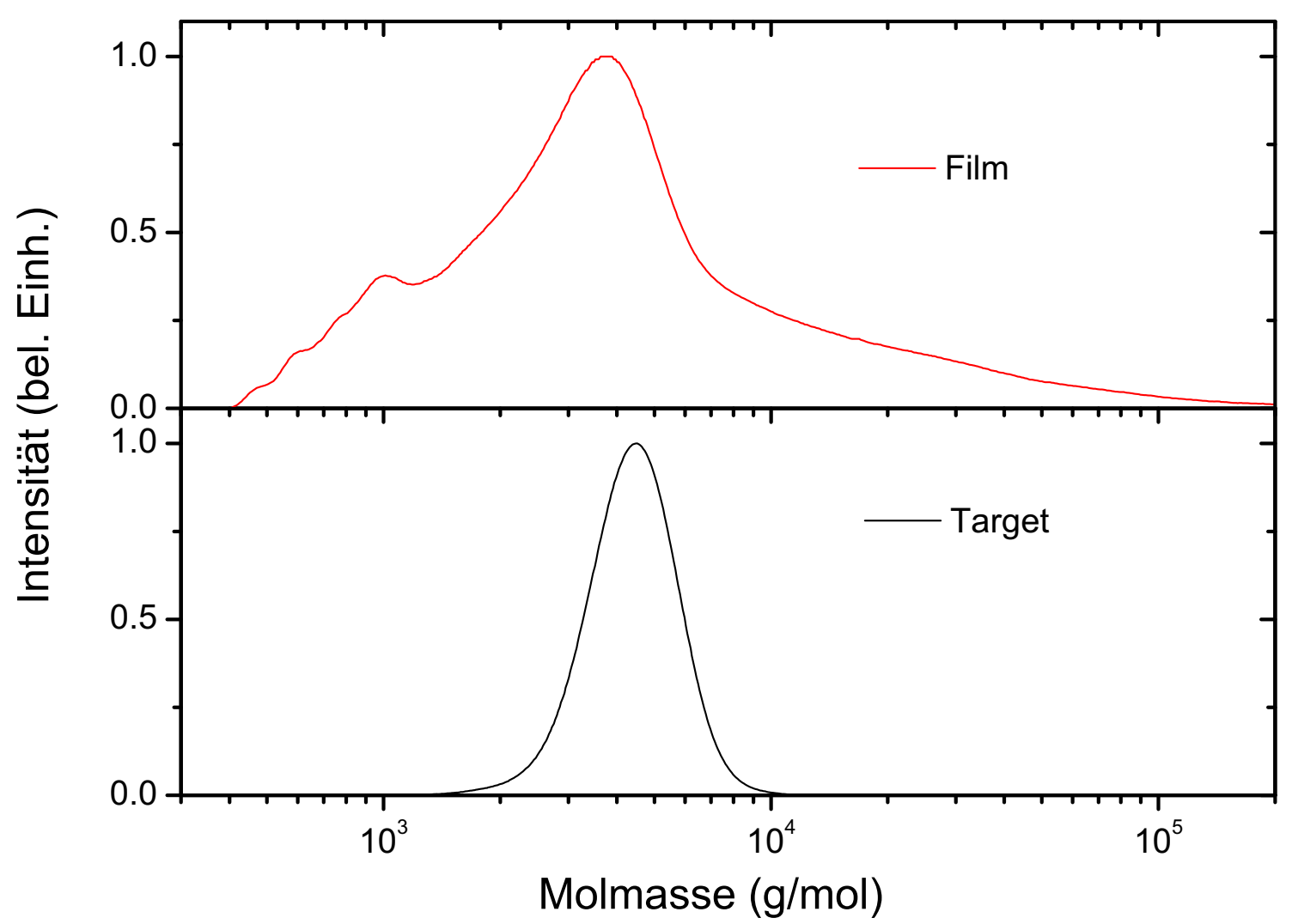

Abbildung 4.15: Chromatogramm eines laserdeponierten Films, der aus einem PMMAEichstandard mit $4.200 \mathrm{~g} / \mathrm{mol}$ hergestellt wurde

sehen, dass der Hauptteil der Moleküle eine recht scharfe, symmetrische Massenverteilung um $4.200 \mathrm{~g} / \mathrm{mol}$ aufweist. Allerdings ist eine breite Verteilung an Molmassen, die bis an $10^{5} \mathrm{~g} / \mathrm{mol}$ heranreicht, klar zu erkennen. Eine scharfe Verteilung von Vielfachen tritt nicht auf. Damit ist die direkte lineare Vernetzung auszuschließen. Übrig bleiben die Möglichkeiten des Wachstums über das Anwachsen kleinerer Bruchstücke wie Monomere, wie sie im Massenspektrum bei der Ablation vorhanden sind (siehe Abschnitt 4.2.1), oder das Wachstum durch zufälliges Vernetzen der Makromoleküle über Seitengruppen. Im letzteren Fall bleiben die Moleküle löslich solange sie nicht zu groß werden. Sie werden von der SEC als größere Moleküle erkannt, da diese ausschließlich die physikalische Größe bestimmt. Da weiterhin die Vernetzung an zufälligen Stellen im Polymerstrang erfolgt, wird die Form des Moleküls verändert. Die SEC ist ausschließlich für lineare Moleküle kalibriert, so dass 
diese Verästelung zu einer verbreiterten Kurve führen sollte. Aus den hier vorgestellten Messungen muss eine Entscheidung zwischen diesen beiden Mechanismen erst einmal offen bleiben, eine weitere Diskussion auf Basis der gemessenen mechanischen Eigenschaften folgt in Kapitel 6 .

\subsection{Zusammenfassung}

Die Laserablation von PMMA kann also als ein mehrstufiger Prozess beschrieben werden. Zuerst tritt mit der Inkubation eine photochemische Veränderung des Polymers auf. An zufälligen Stellen koppelt ein Photon in eine Carbonylbindung ein und die Seitengruppe wird abgespalten oder zumindest verändert, was zur Spaltung der Hauptkette führen kann. Auf diese Weise entstehen statistisch zusätzliche Doppelbindungen, die die Absorption lokal erhöhen. Dadurch steigt die Temperatur durch stärkere Absorption in diesem Bereich stark an und es kommt zur photothermischen Zersetzung der Umgebung [103]. Bildung von flüssigem PMMA und zusätzlicher gasförmiger Komponenten führen zur Bildung von Blasen und deren Platzen, was die Hauptquelle der abgetragenen Masse im hier betrachteten Bereich der Fluenz ist. Dies ist dann der Fall, wenn der Absorptionskoeffizient einen kritischen Wert überschritten und gleichzeitig die mittlere Molmasse und damit der Schmelzpunkt einen kritischen Wert unterschritten haben. Das Material wird abgetragen und der Mechanismus setzt sich in tiefer liegenden Ebenen fort.

Die Verstärkung der Absorption durch die chemische Veränderung stellt damit automatisch eine Instabilität dar, die notwendigerweise zur Kettenlängenverkürzung und DropletBildung führt. Eine Idee diese zu umgehen ist, Energien direkt oberhalb der Ablationsschwelle zu verwenden, was viele experimentelle Nachteile wie geringe Depositionsraten und schlechte Prozesskontrolle ergibt. Andere, vielversprechendere Möglichkeiten werden in Kapitel 5 diskutiert. 


\section{Modifikation der gepulsten Laserdepositon durch Targetbeigaben}

Auch wenn vor allem die besonderen mechanischen Eigenschaften laserdeponierter Filme interessant sind [120], ist für viele Anwendungen der in Kapitel 4 beschriebene Mechanismus der gepulsten Laserdeposition von PMMA mit dem damit verbundenen Ergebnis mit den hohen Rauigkeiten durch die Droplets und den stark verkürzten Ketten unbefriedigend. Vor allem für die Perspektive der Schichtherstellung komplexerer organischer Materialien ist außerdem eine deutliche Verringerung der Zerstörung des Materials während der Ablation und Deposition zwingend notwendig. Um den Ablationsmechanismus zu verändern, liegt es nahe, die Absorptionstiefe der UV-Strahlung im Target zu erhöhen, um die Inkubationsphase zu überspringen. Dadurch sollte die natürliche Instabilität des Prozesses verringert und die Tröpfchenbildung auf das für PLD Filme anderer Materialklassen übliche Maß [33] reduziert werden.

\subsection{Antracen}

Die erste nahe liegende Maßnahme zur Erhöhung der Absorption ist der Zusatz eines UVLicht absorbierenden Materials zum Target. Dieses sollte sehr stark absorbieren, damit kleine Mengen ausreichen, eine große Wirkung zu erzielen. Damit keine Rückstände im Film auftreten sollte es durch die Bestrahlung vollständig zersetzt werden (siehe z. B. [51, 89]). In der Literatur wird das organische Molekül Antracen verwendet [129, 126] (Strukturformel siehe Abb. 5.1), der zweiteinfachste polyzyklische aromatische Kohlenwasserstoff. Es
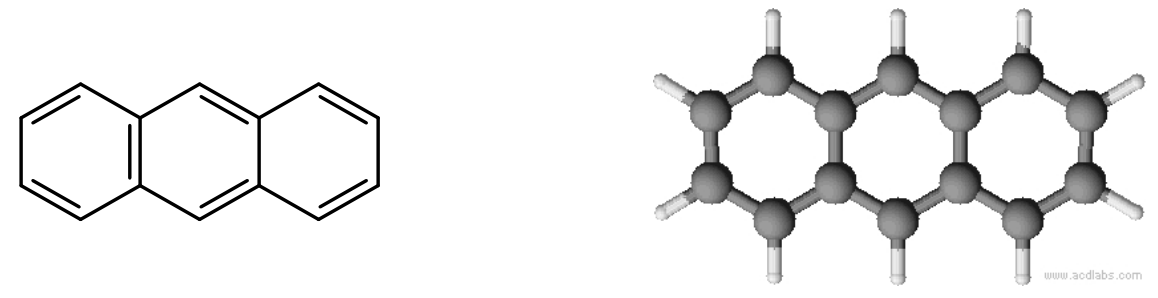

Abbildung 5.1: Strukturformel von Antracen

wurde erfolgreich für eine Reihe von Materialien verwendet, wenn auch hauptsächlich zum 
Abtragen durch Laser höherer Wellenlänge, meist $308 \mathrm{~nm}$ [40]. Tsuboi et al. [127, 128] berichten damit sogar über die Deposition von dünnen Filmen aus Seidenprotein mit $351 \mathrm{~nm}$ und $355 \mathrm{~nm}$ Lasern.

Durch Kugelmühlen und pressen (siehe Abschnitt 2.1.2) wurden Targets mit 0, 3 und 10 Gew.\% Antracen-Beimengung hergestellt . Alle in diesem Abschnitt gezeigten Experimente wurden bei einer Laserenergiedichte von $1,2 \mathrm{~J} / \mathrm{cm}^{2}$ durchgeführt. Schon ein erster Blick auf die mit Antracen versetzten Targets während der Bestrahlung zeigt einen deutlichen Unterschied zum reinen PMMA. Bilder vom Ablationsprozess von drei Targets mit unterschiedlicher Antracen-Konzentration, jeweils beim 100. Puls auf eine Position, sind in Abbildung 5.2 gezeigt. Für reines PMMA sieht man einen kleinen, blau fluoreszieren-
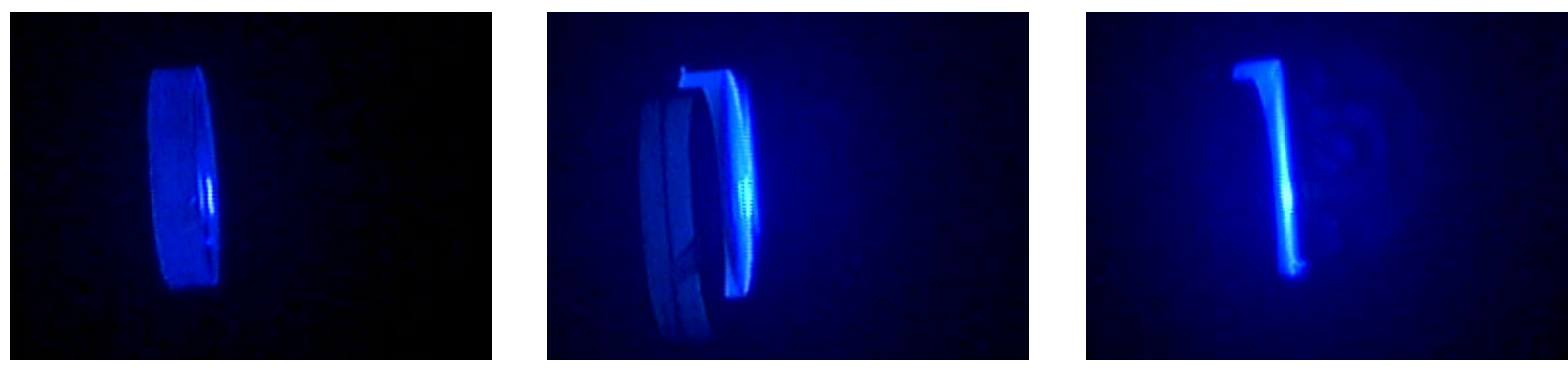

Abbildung 5.2: Bild des laserbestrahlten Targets für die verschiedene TargetZusammensetzung jeweils beim 100. Schuss auf eine Position. Von links nach rechts: reines PMMA, PMMA +3\% Antracen, PMMA +10\% Antracen.

den Punkt ohne erkennbare Plasmabildung. Mit steigendem Antracen-Gehalt wird dieser Punkt deutlich großflächiger und heller, d.h. die Absorption des UV-Lichts ist deutlich stärker. Besonders für die Probe mit 10\% Antracen sind sogar Ansätze für eine Plasmabildung zu erkennen, auch wenn diese bei weitem nicht so ausgeprägt ist wie für metallische Targets 1 .

Auch für diese Targets wurde jeweils eine Aufnahmeserie der ersten 100 Pulse auf eine frische Position gemacht. Diese ist in Abbildung 5.3 für $3 \%$ und 5.4 für $10 \%$ AntracenZugabe gezeigt. Im unteren Teil der Abbildungen ist jeweils wieder die Helligkeit des Farbkanals Rot über die Einzelbilder integriert und gegen die Pulszahl aufgetragen. Es ist deutlich zu sehen, dass der Inkubationsprozess deutlich weniger ausgeprägt ist als für reines PMMA (Vergleiche Abbildung 4.5 auf Seite 45). Vor allem für die Probe mit 3\% Antracen

\footnotetext{
${ }^{1}$ Zum genauen Verständnis der Bilder ist zu beachten, dass die drei Targets gleichzeitig übereinander in die Kammer eingebaut und nacheinander in den Laserstrahl gefahren wurden. Deshalb ist für das mittlere Bild ein leichtes Fluoreszieren des darüber befindlichen PMMA-Targets zu sehen und beim rechten Bild sieht man den durch das darüber liegende Target abgedeckten Bereich und rechts neben dem beleuchteten Fleck Schrauben des Targethalters.
} 


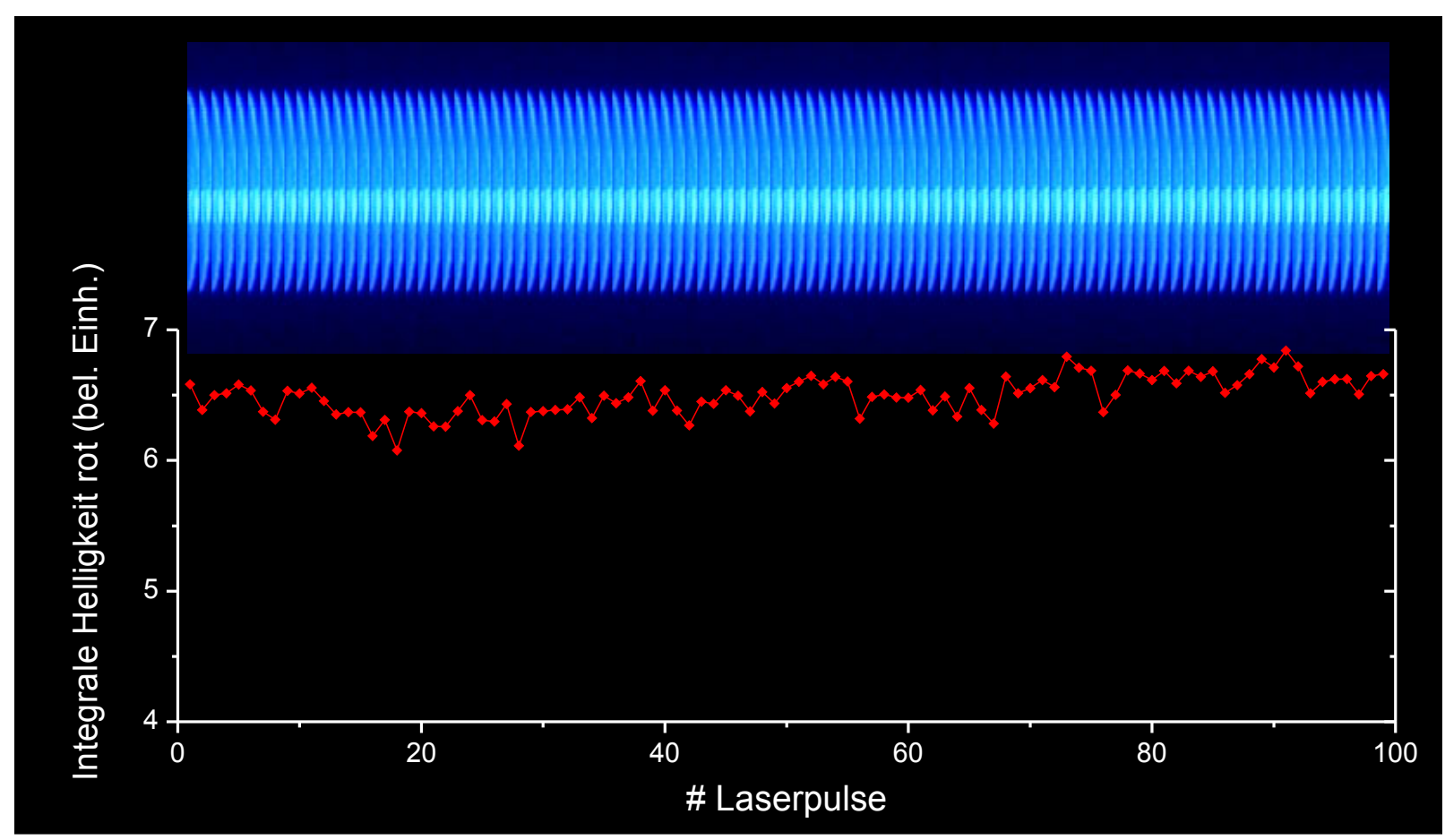

Abbildung 5.3: Oben: Entwicklung der Laserbestrahlung von PMMA mit 3\% Antracen während der ersten 100 Laserpulse; Unten: Integral über die Helligkeit der Farbe Rot

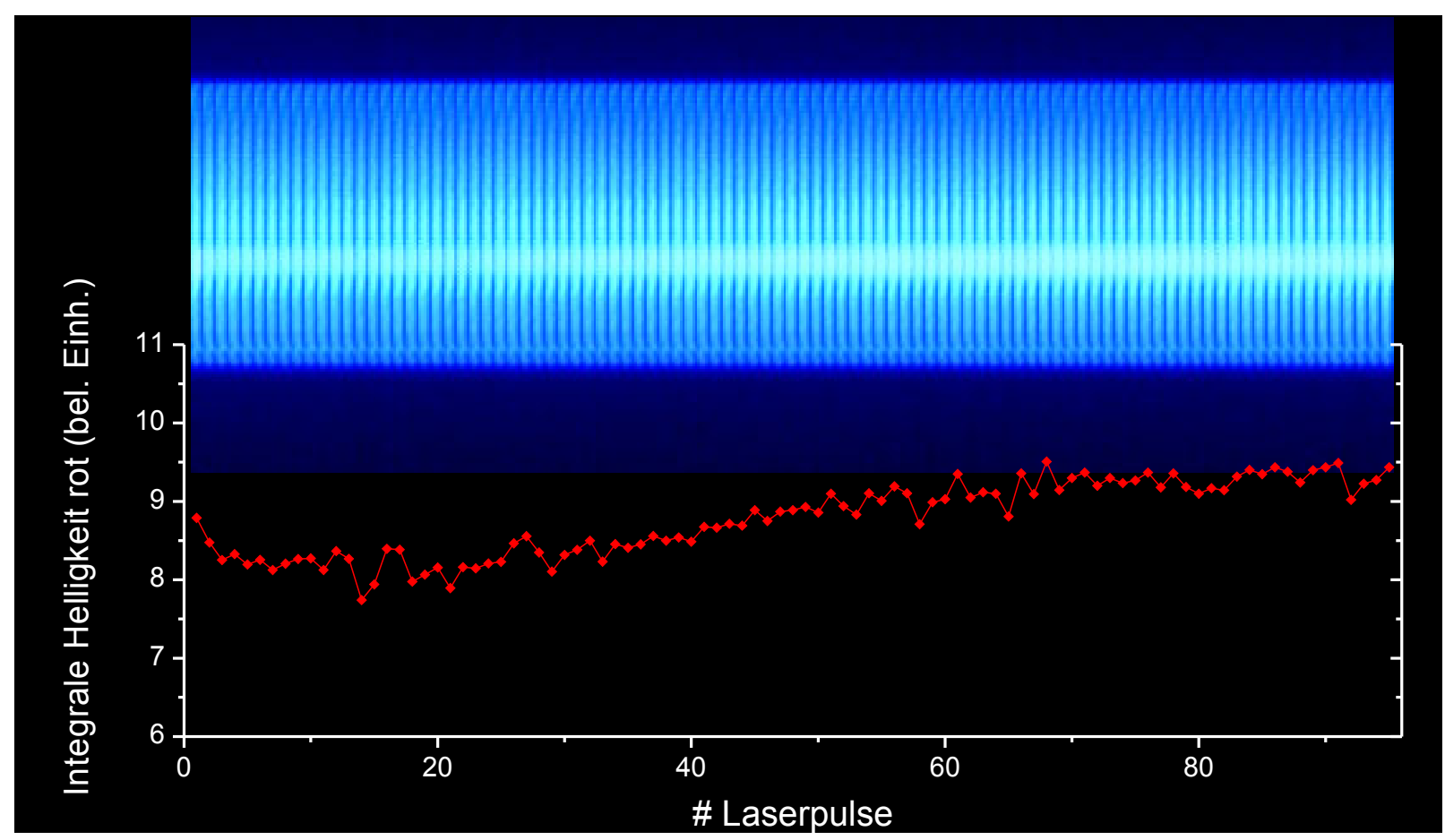

Abbildung 5.4: Oben: Entwicklung der Laserbestrahlung von PMMA mit 10\% Antracen während der ersten 100 Laserpulse; Unten: Integral über die Helligkeit der Farbe Rot 
ist keine Veränderung der Bilder mit der Pulszahl zu erkennen. Für 10\% Antracen-Zugabe ist eine leichte Zunahme der Intensität fast über den gesamten Zeitraum von 100 Pulsen sichtbar. Diese ist möglicherweise darin begründet, dass das Target recht tief abgetragen und damit der bestrahlte Bereich vergrößert wird. Die Zunahme ist hier jedoch sehr viel geringer als bei reinem PMMA, obwohl die Abtragstiefe vergleichbar hoch ist.

Einen weiteren deutlichen Hinweis auf die Vermeidung der Inkubationsphase findet man bei der Betrachtung der Depositionsraten. Hierfür wurde das von drei Targets mit unterschiedlichem Antracen-Gehalt abgetragene Material direkt auf eine Schwingquarzwaage deponiert. Die gemessenen Dicken sind in Abbildung 5.5 aufgetragen. Wie zu sehen ist,

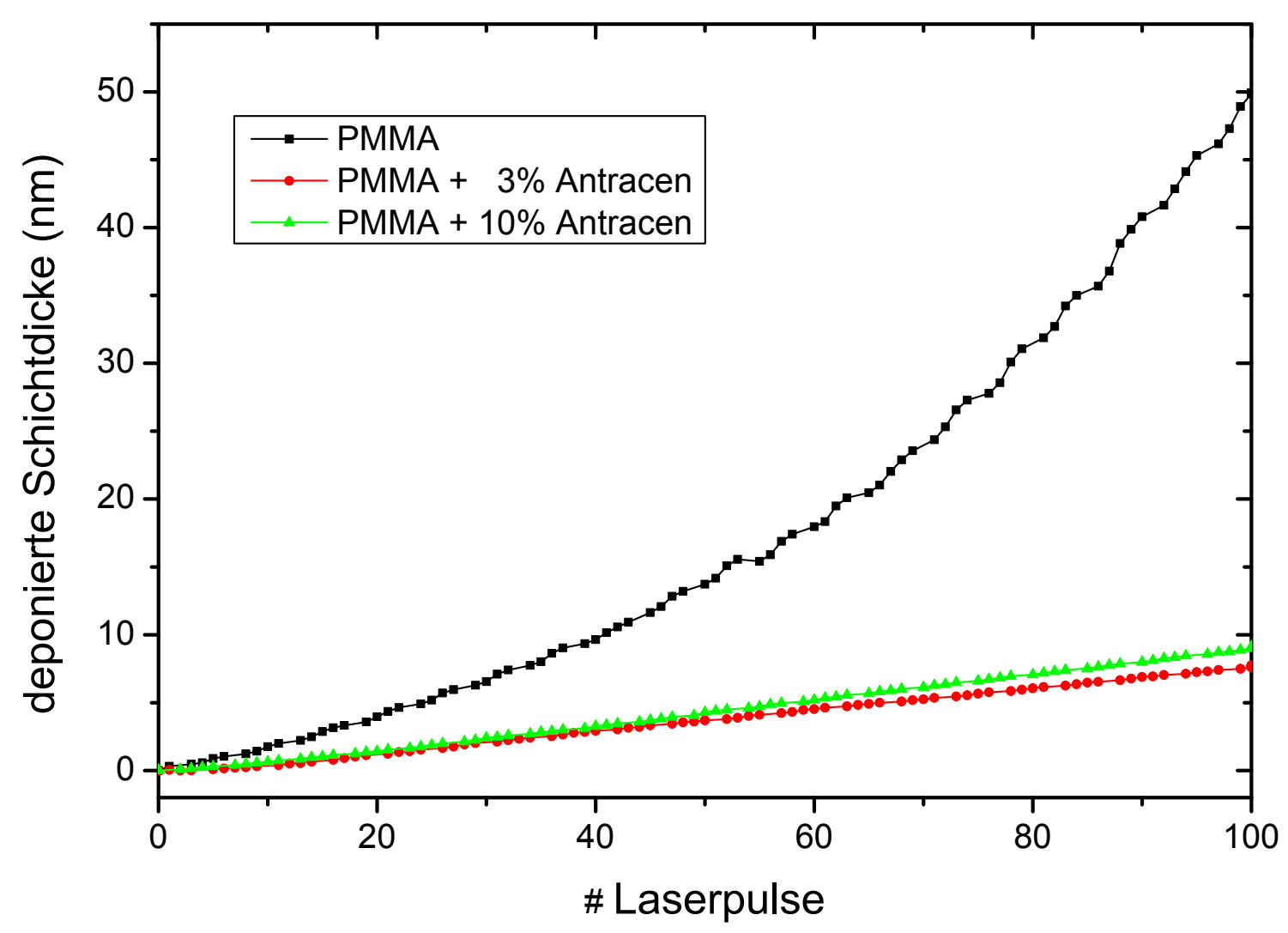

Abbildung 5.5: Vergleich der deponierten Filmdicken für verschiedenen Antracen-Gehalt unter sonst identischen Bedingungen

wird der bei reinem PMMA gefundene Bereich verminderter Depositionsrate vermieden. Für eine Antracen-Konzentration von 3\% im Target setzt die Deposition von Material ab dem fünften, für 10\% schon ab dem zweiten mit konstanter Rate ein. Dafür ist die Depositionsrate im Gleichgewicht bis zu eine Größenordnung niedriger als für reines PMMA. Da 
ein großer Teil der deponierten Masse auf Droplets zurück zu führen ist, erwartet man hier schon eine starke Reduktion der Droplet-Bildung. Die Raten sind für Proben mit 3 und 10\% Antracen fast identisch und der Anstieg ist völlig linear, wie es für den glatten Anteil von Filmen aus reinem PMMA schon gezeigt wurde [92].

Betrachtet man die Targets nach dem Beschuss, so ist festzustellen, dass sich die Morphologie, im Besonderen die Kraterbildung, deutlich unterscheidet. Nach 1000 Pulsen auf reines PMMA ergibt sich ein Loch mit ca. 3,1 mm Tiefe und deutlich ausgefranstem Boden, was vermutlich auf Inhomogenitäten im Energieprofil des Lasers im Zusammenspiel mit der natürlichen Instabilität im Ablationsprozess zurückzuführen ist. Bereits bei niedrigeren Pulszahlen ist das Material am Rand stärker abgetragen als im Zentrum. Dieses wurde schon von Ghosh et al. [44 beschrieben und durch hohe Geschwindigkeiten des abgetragenen Materials und damit verbundene Abschattung des Laserstrahls bei höheren Energiedichten erklärt. Da die Effekte auch bei Verwendung eines Strahlhomogenisierers beobachtet wurden, sind Inhomogenitäten im Laserstrahl vermutlich auszuschließen. Weiterhin denkbar wären auch Reflektionen und damit verbundene Verstärkung der Laserstrahlung an den Kanten. Bei Targets mit 3\% Antracen-Anteil ist der abgetragene Bereich wesentlich flacher, nur ca. 0,1 mm tief, und sehr gleichmäßig. Bei Zugabe von 10\% Antracen erhält man nach 1000 Pulsen einen 0,9 mm tiefen Krater, der jedoch im Unterschied zu reinem PMMA scharfe Kanten aufweist, und einen flachen Boden. Offensichtlich findet hier eine erheblich gleichmäßigere Ablation statt. Es ist anzumerken, dass bei nur 100 Pulsen auf reines PMMA ein homogenes Loch entsteht, das ca. 0,2 $\mathrm{mm}$ tief ist. Dies ist dadurch zu erklären, dass etwa die ersten 70 Pulse benötigt werden, um die Inkubationsphase zu vervollständigen und die Ablation während dieser Phase deutlich reduziert ist (vgl. Abb. 5.5).

Die Mikrostruktur bestrahlter Targets unterschiedlichen Antracen-Gehalts ist auf den rasterelektronenmikroskopischen Bildern in den Abbildungen 5.65.8 zu sehen. Die Targets wurden unter gleichen Bedingungen jeweils mit 100 Pulsen bestrahlt.
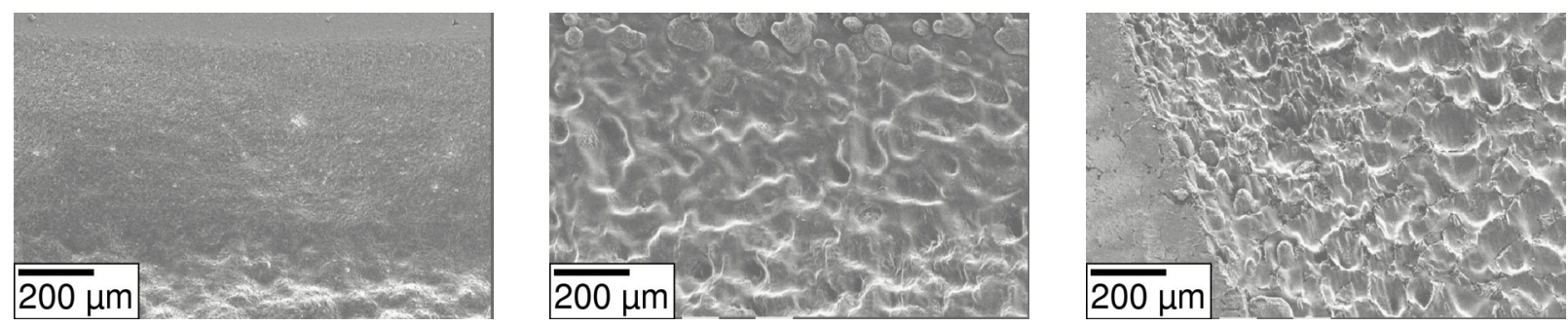

Abbildung 5.6: Targets bestrahlt mit 100 Pulsen bei $60 \mathrm{~mJ}$. Von links nach rechts: PMMA ohne, mit 3\% und mit 10\% Antracen bei ca. 50facher Vergrößerung 
In den Übersichtsaufnahmen in Abbildung 5.6 ist zu erkennen, dass die mit Antracen modifizierten Targets am Grund des abgetragenen Bereichs eine deutlich größere makroskopische Aufrauung der Oberfläche aufweisen als das reine PMMA. Die Zapfenbildung gleicht der bei einigen Metallen wie etwa Permalloy [98]. Dies lässt vermuten, dass die Oberfläche thermisch sehr stark verändert wurde, vermutlich partiell aufgeschmolzen und danach wieder erstarrt ist. Im Gegensatz dazu ist die Oberfläche des reinen Polymertargets vergleichsweise glatt mit gleichmäßig, statistisch verteilten Löchern.
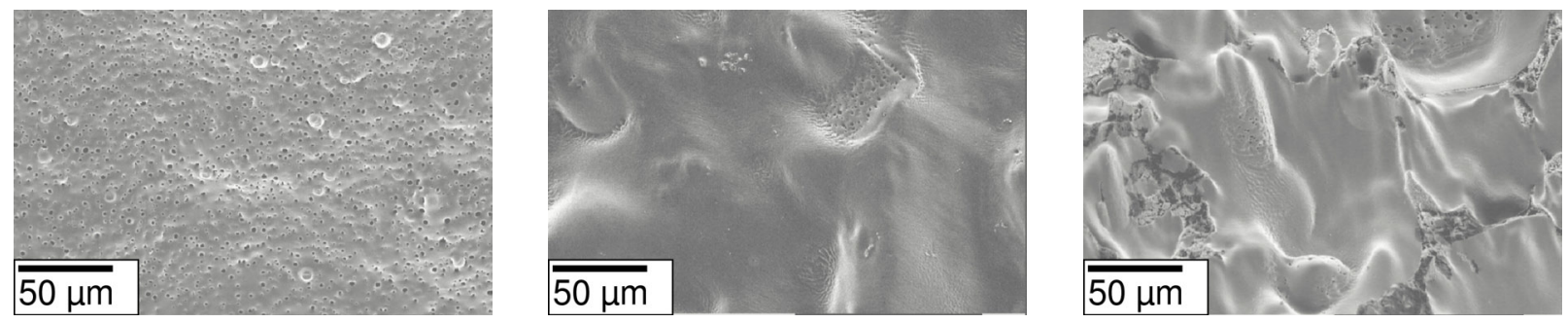

Abbildung 5.7: Targets bestrahlt mit 100 Pulsen bei 60 mJ. Von links nach rechts: PMMA ohne, mit 3\% und mit 10\% Antracen mit ca. 200facher Vergrößerung

Bei den Bildern mit höherer Vergrößerung in Abbildung 5.7 ist zu sehen, dass die Struktur für die Targets mit Antracen Inhomogenitäten aufweist. Die makroskopische Struktur der Zapfen enthält einzelne Bereiche, die mit Löchern übersäht sind wie für die reinen PMMA-Targets.
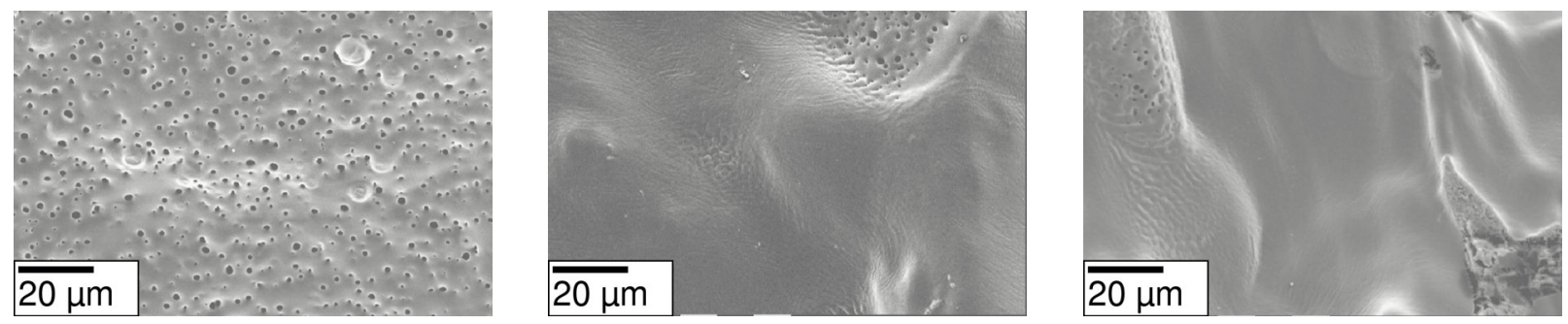

Abbildung 5.8: Targets bestrahlt mit 100 Pulsen bei $60 \mathrm{~mJ}$. Von links nach rechts: PMMA ohne, mit 3\% und mit 10\% Antracen mit ca. 500facher Vergrößerung

In der höchsten Vergrößerung in Abbildung 5.8 kann man auszählen, dass die Dichte der Löcher in den schwammartigen Stellen der Proben mit Antracen um einen Faktor 2-3 höher ist als in den reinen PMMA-Targets.

Betrachtet man die aus den drei Targets deponierten Filme, kann man erhebliche Unterschiede feststellen. Rasterelektronenmikroskopische Bilder sind in den Abbildungen 5.9 und 5.10 gezeigt. Für die Herstellung dieser Filme wurden je 300 Pulse auf die gleiche Position des Targets abgegeben ohne dieses zu bewegen. Die Filme von reinen PMMA-Targets 

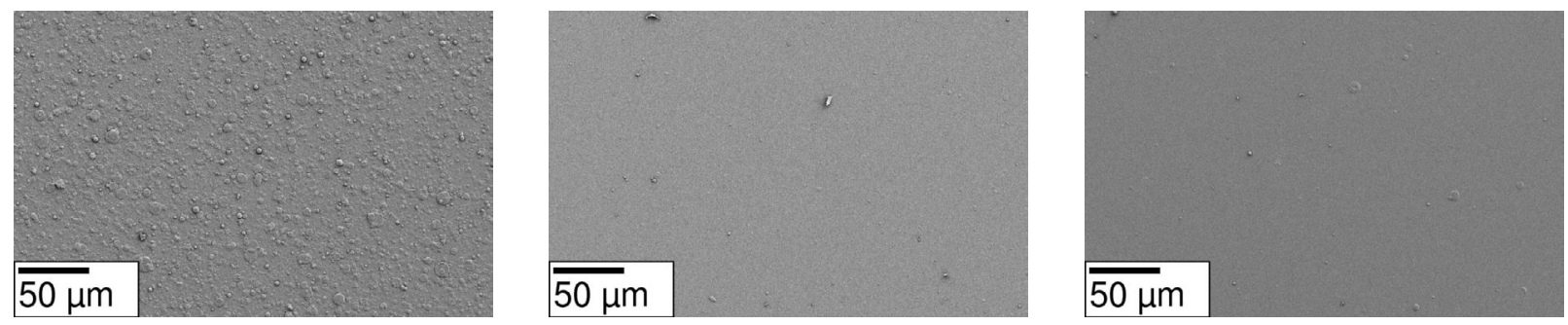

Abbildung 5.9: Filme mit 300 Pulsen bei $60 \mathrm{~mJ}$ hergestellt. Von links nach rechts: PMMA ohne, mit 3\% und mit 10\% Antracen mit ca. 150facher Vergrößerung
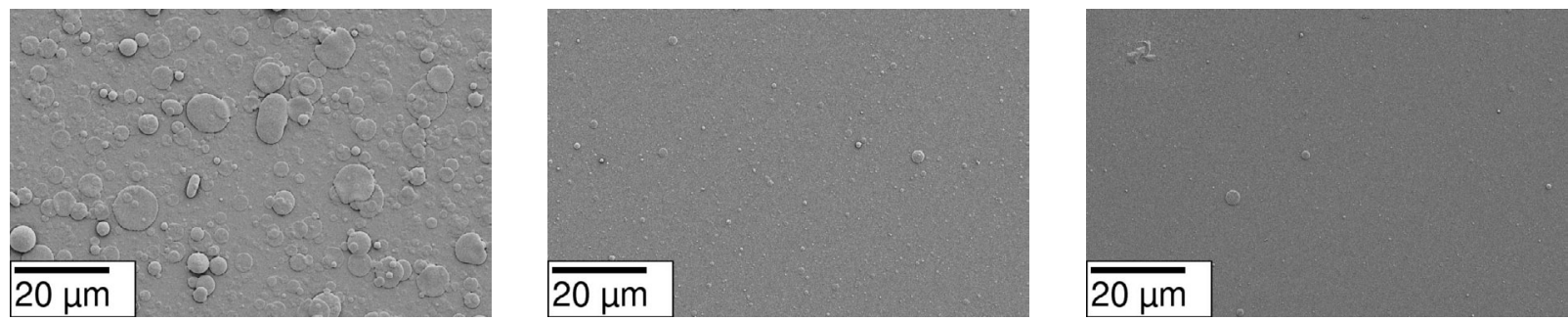

Abbildung 5.10: Filme mit 300 Pulsen bei $60 \mathrm{~mJ}$ hergestellt. Von links nach rechts: PMMA ohne, mit 3\% und mit 10\% Antracen mit ca. 500facher Vergrößerung

zeigen die aus Kapitel 4 übliche Struktur, eine fast geschlossene Schicht an Tröpfchen von einigen $\mu \mathrm{m}$ Größe. Die mit Antracen angereicherten Targets ergeben dagegen beide deutlich glattere Filme und eine sehr geringe Anzahl großer Tröpfchen. Dabei ist zu bedenken, dass die deponierte durchschnittliche Schichtdicke bei 300 Pulsen erheblich geringer ist, als für reines PMMA. Bei großen Vergrößerungen ist zu sehen, dass diese Filme mit Tröpfchen bedeckt sind, allerdings sind diese sehr viel kleiner, wenngleich sehr häufig.

Da besonders die Filme mit 10\% Antracen im Targetmaterial von den normalen PMMAFilmen abweichen, wurden von deren Oberflächenmorphologie rasterkraftmikroskopische Aufnahmen gemacht um quantitative Höheninformationen zu erhalten (siehe Abbildung 5.11). Im linken Bild ist hier zu sehen, dass die Oberflächenmorphologie mit der der Rasterelektronenmikroskopischen Aufnahmen vergleichbar ist. Die Höhe der Droplets beträgt maximal etwa 60-70 nm, der maximale Durchmesser dabei etwa 120-130 nm. Die obere rechte Darstellung ist bestmöglich unverzerrt dargestellt und zeigt, dass die großen Droplets tatsächlich als Halbkugeln auf der Oberläche liegen, die kleineren sind etwas flacher. Das zum Vergleich dargestellte hoch aufgelöste Rasterelektronenmikroskopbild von kleinen Tröpfchen auf derselben Probe, wofür die Probe um $75^{\circ}$ in Richtung des Detektors gekippt wurde, bestätigt die fast halbkugelförmige Struktur der Droplets und auch, das diese mit der Oberfläche benetzen und nicht als Rotationsellipsoid aufliegen. Wie auch für die Fil- 


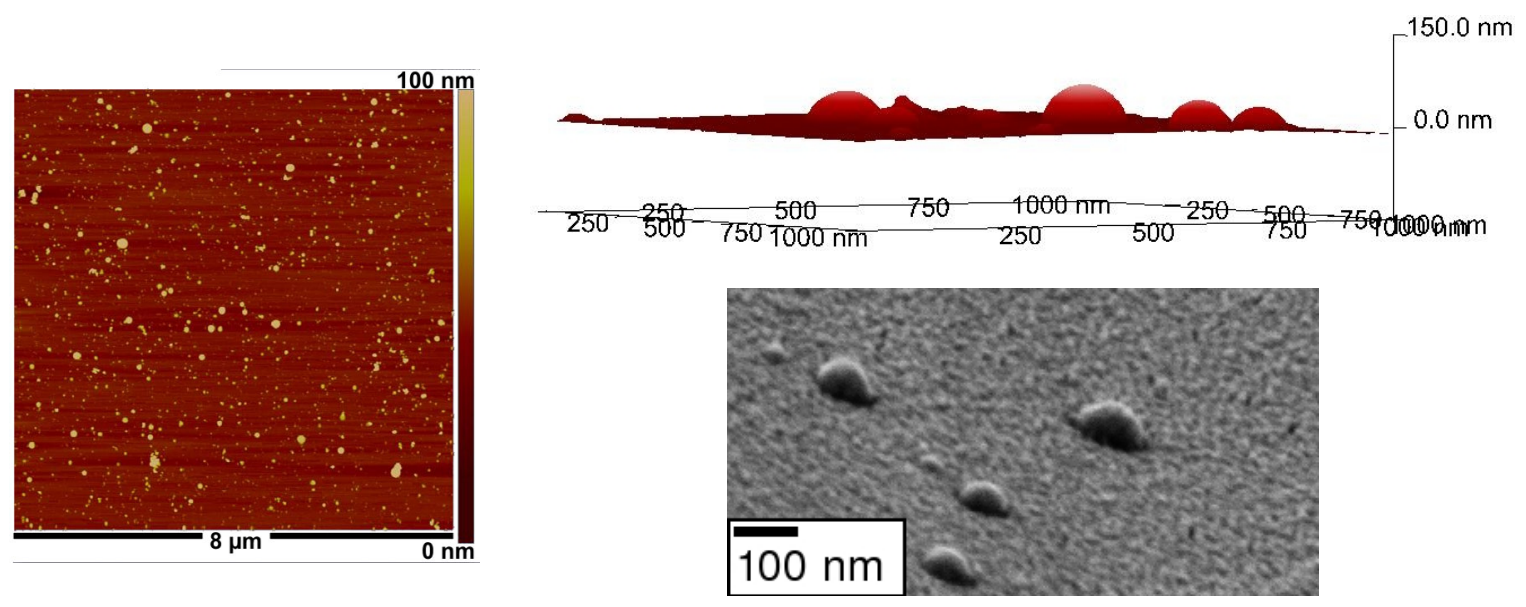

Abbildung 5.11: Links und oben rechts: Rasterkraftmikroskopbilder von Filmen aus PMMA mit 10\% Antracen, 300 Pulse. Es sind zwei verschiedene Vergrößerungen gezeigt. Unten rechts: Rasterelektronenmikroskopbild der selben Probe, um $75^{\circ}$ zum Detektor gekippt aufgenommen

me aus reinen PMMA-Targets konnte für diese Probe, die mit 300 Laserpulsen deponiert wurde, mittels Röntgenreflektometrie eine glatte Komponente nachgewiesen werden, die im Fall dieser Schicht 34,5 nm dick ist. Dies ist signifikant mehr, als für die Schichten, die aus reinen PMMA-Targets hergestellt wurden. Dort wurden mit 500 Pulsen nur 18,4 nm glatte Schicht deponiert (siehe Abschnitt 4).

Zur Klärung der chemischen Zusammensetzung und der Frage, ob neben dem PMMA auch Antracen deponiert wurde, wurden Infrarotspektren aufgenommen (siehe Abbildung 5.12. Zusätzlich ist das Spektrum von reinem Antracen aufgetragen. Es ist zu sehen, dass die Filme die charakteristischen Linien für PMMA zeigen. Abweichungen in der Stärke der Banden sind hauptsächlich auf die Grundlinienkorrektur der BRUKER Software zurückzuführen, da die Schichten sehr dünn waren. Vor allem ist festzustellen, dass keine der für Antracen charakteristischen Linien in den Spektren der Filme signifikant nachzuweisen ist. Somit ist davon auszugehen, dass Antracen durch die Laserstrahlung weitgehend zerstört wird. Auch die von Tsuboi et al. [126] berichteten Veränderungen der der $\mathrm{CH}$ und $\mathrm{C}=\mathrm{O}$ Streckbanden des PMMA, die von den Autoren auf eine Vernetzung über die $\mathrm{CH}_{2}$ Gruppe des Hauptstrangs zurückgeführt wurde, ist nicht nachzuvollziehen, wenngleich nicht völlig auszuschließen.

Eine weitere Veränderung des Prozesses durch die Beimengung von Antracen zeigt sich in der Kettenlänge des Filmmaterials. Die Chromatogramme der deponierten Filme sind in Abbildung 5.13 gezeigt. Es ist zu sehen, dass der erwünschte Effekt einer verringerten 


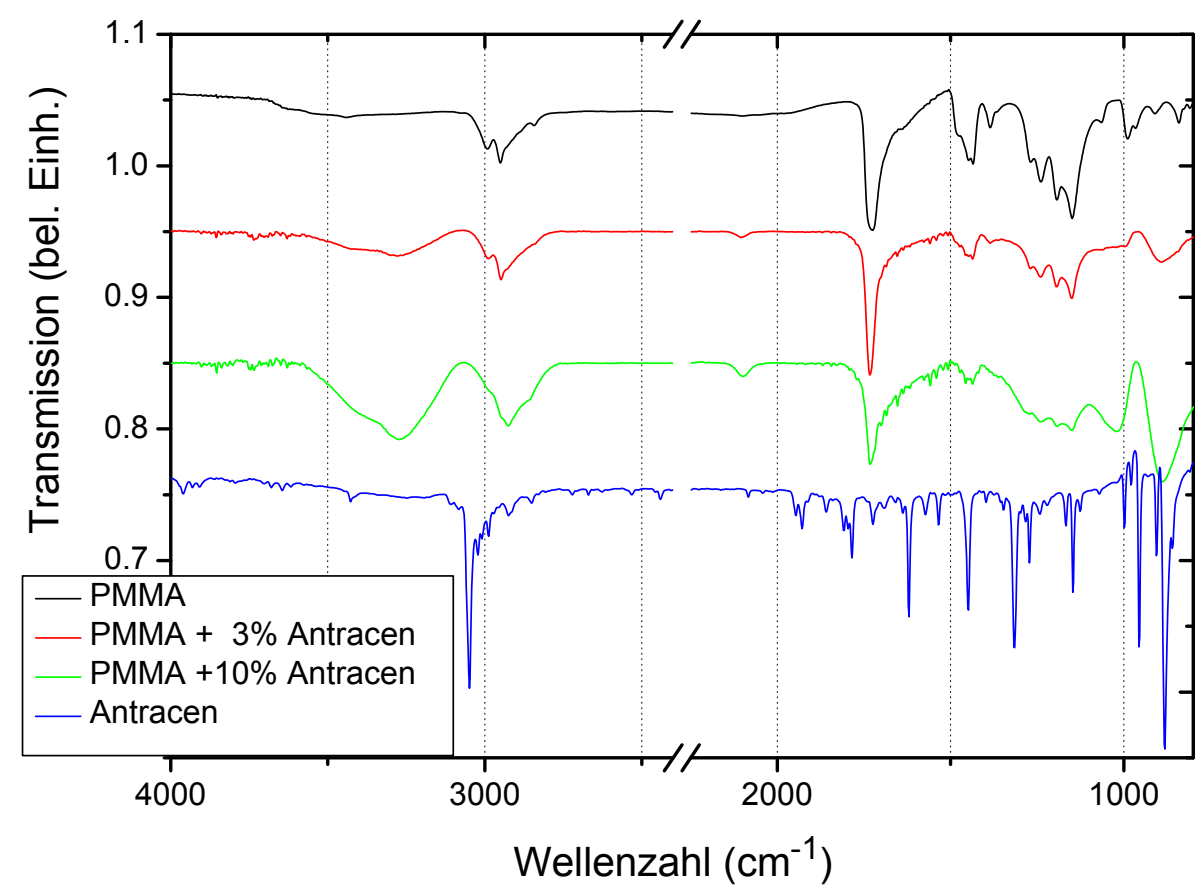

Abbildung 5.12: Infrarotspektren von Filmen mit unterschiedlichem Antracen-Gehalt im Targetmaterial und von Antracen. Die Kurven wurden für bessere Übersichtlichkeit vertikal gegeneinander verschoben.

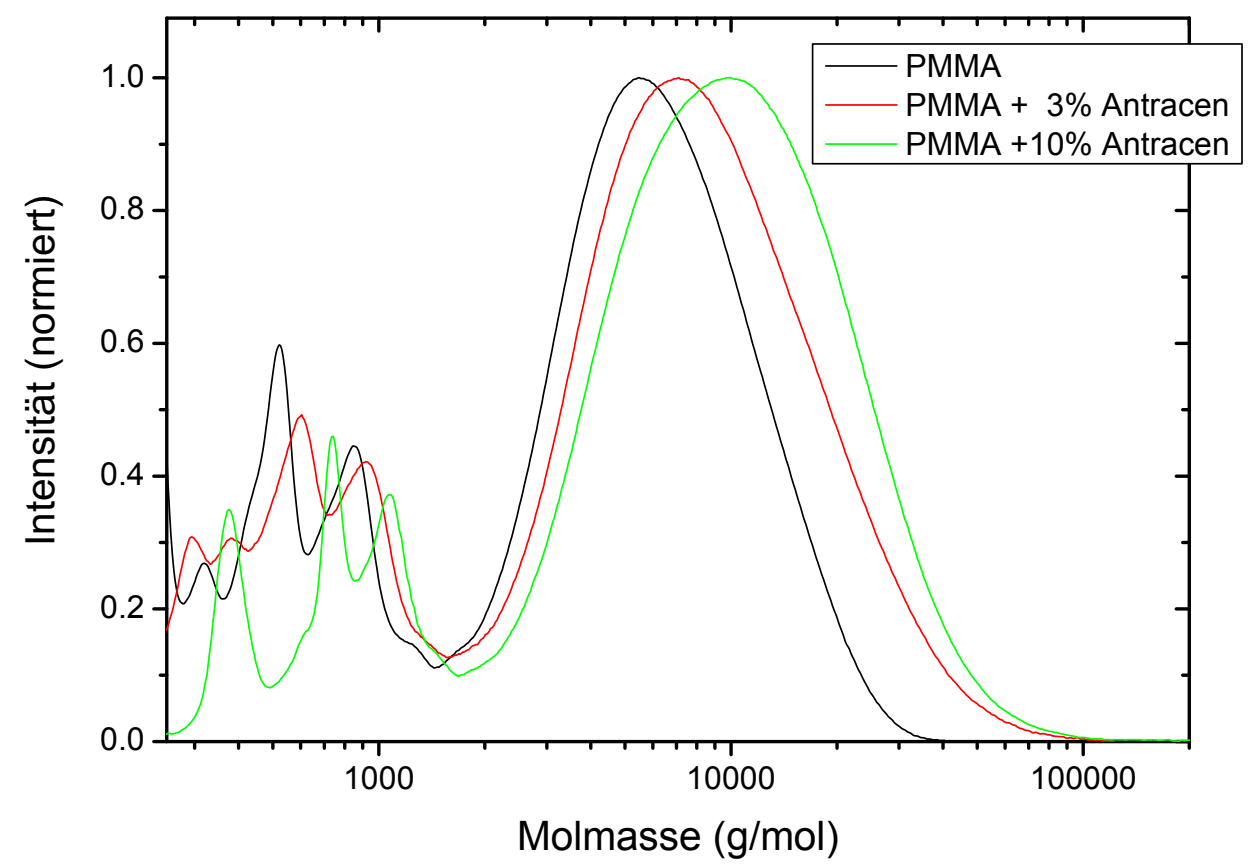

Abbildung 5.13: SE-Chromatogramme von Proben mit 0\%, 3\% und 10\% Antracen im Targetmaterial 
Zerstörung teilweise eintritt, die Kettenlänge steigt mit steigendem Antracengehalt von $M_{W}=8.000 \mathrm{~g} / \mathrm{mol}\left(M_{N}=5.300 \mathrm{~g} / \mathrm{mol}\right)$ bis auf $M_{W}=10.500 \mathrm{~g} / \mathrm{mol}\left(M_{N}=7.000 \mathrm{~g} / \mathrm{mol}\right)$ bei 3 und $M_{W}=14300 \mathrm{~g} / \mathrm{mol}\left(M_{N}=8.300 \mathrm{~g} / \mathrm{mol}\right)$ bei $10 \%$ Antracen-Zugabe. Diese Veränderung ist deutlich, allerdings zu gering um zu erwarten, dass grundlegende Veränderungen im Prozess mit dem Effekt des Übertrags eines weitgehend unbeschädigten Polymers auf diese Weise möglich ist, da bei höherem Antracen-Gehalt auch ein Übertrag von Antracen zu erwarten ist [73].

Insgesamt stellt man fest, dass die Beimengung von Antracen zu den PMMA-Targets die Absorption deutlich modifiziert. Die Effizienz der Ablation nimmt deutlich zu, das polymere Material wird durch die Absorption im Antracen weniger beschädigt. Die Tröpfchenbildung wurde verringert, aber nicht völlig unterbunden, und die erreichte Kettenlänge ist immer noch weit entfernt von der des Ausgangsmaterials. Eine mögliche Ursache hierfür ist die durch das Kugelmühlen schlecht kontrollierbare Verteilung des Antracens in dem Polymer. Auch wenn diese makroskopisch homogen ist, können sich mikroskopisch immer noch kleine Körner ergeben, die weiterhin verhältnismäßig große Absorptionszentren bilden und die Tröpfchen verursachen. Für diese Interpretation sprechen vor allem die Bilder der Mikrostruktur der Targets, auf denen gezeigt werden konnte, dass glatte Bereiche auftreten und Bereiche, die mit Löchern übersäht sind wie die reinen PMMA-Targets. Der grundsätzliche Depositionsmechanismus wird damit also nur schwach verändert.

\subsection{UV absorbierender RAFT-Agent}

Eine Idee eine homogene Verteilung des Absorbers zu erzwingen ist es, an jede Polymerkette chemisch eine absorbierende Endgruppe anzuhängen. Dieses wird durch die RAFTPolymerisation (siehe Abschnitt 2.1.2 realisiert, wodurch gleichzeitig Polymere mit scharfer Kettenlängenverteilung erreicht werden. Der verwendete RAFT-Agent, 2-Cyanoprop2-yldithiobenzoat, ist selbst stark UV-absorbierend. Die Strukturformel des so erhaltenen Polymers ist in Abb. 5.14 gezeigt. In diesem Fall wurde RAFT-polymerisiertes PMMA mit einer Molmasse von $M_{W}=16.000 \mathrm{~g} / \mathrm{mol}$ verwendet ohne zusätzliche Änderungen durchzuführen. Die daraus gepressten Targets sind hellrot, transparent und sehr spröde.

Bilder eines mit dem Laser bestrahlten Targets (Zusammenstellung der ersten 100 Pulse in Abb.5.15) zeigen Unterschiede zu denen von reinem PMMA (vgl. Abb. 4.5). Wiederum ist der erste Puls ausgezeichnet, da hier Adsorbate abgelöst werden, was eine größere Leuchterscheinung verursacht als die Ablation des eigentlichen Targets. Mit steigender 


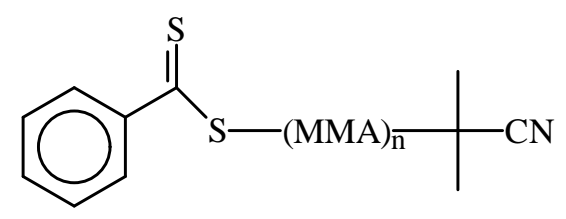

Abbildung 5.14: Strukturformel des mittels RAFT-Polymerisation hergestellten Polymers

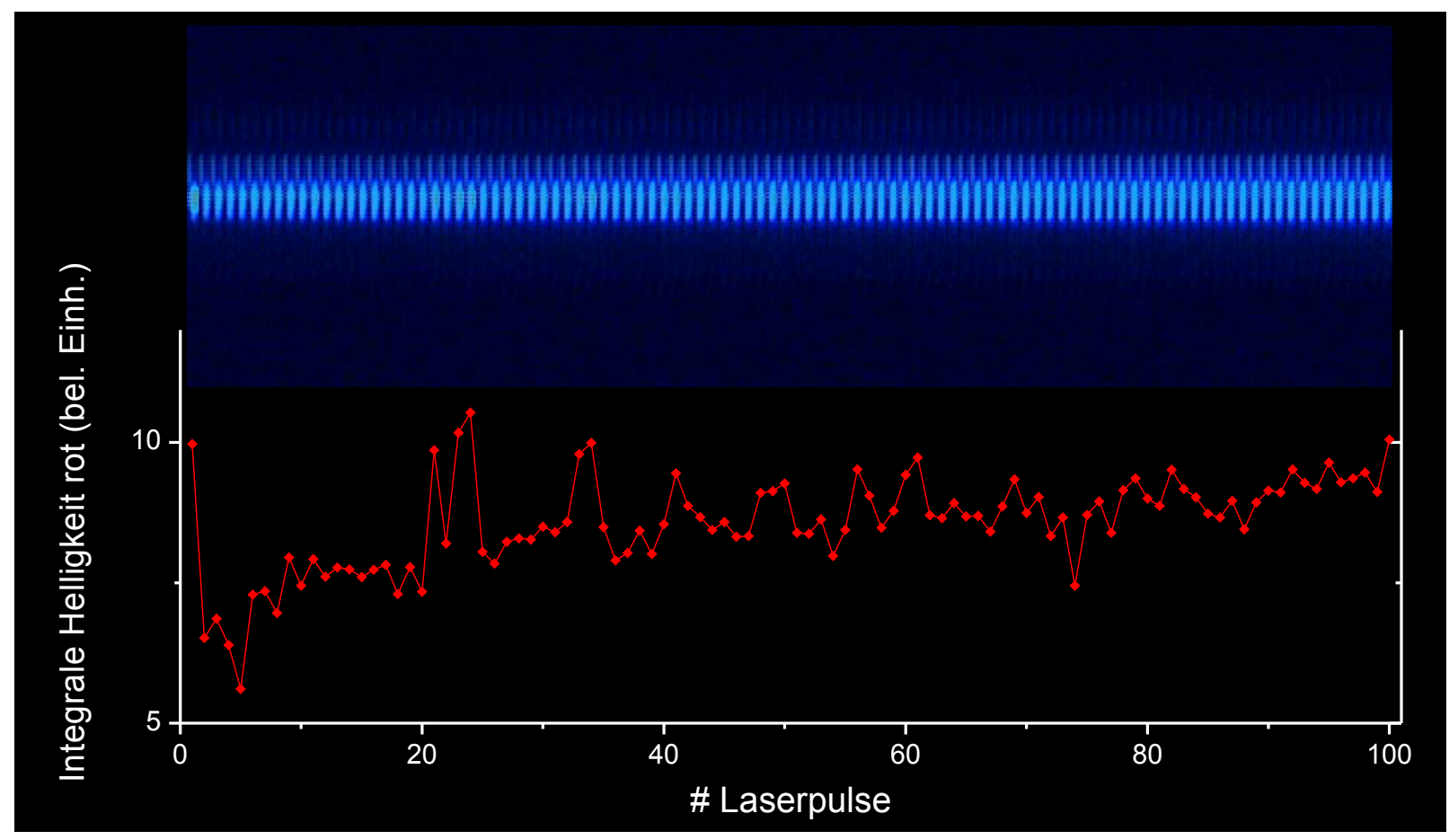

Abbildung 5.15: Oben: Entwicklung der Laserbestrahlung von RAFT-polymerisiertem PMMA während der ersten 100 Laserpulse; Unten: Integral über die Helligkeit der Farbe Rot

Pulszahl ist ein leichter Anstieg der Intensität über etwa 30-40 Pulse zu sehen. Auch in dieser Abbildung ist im unteren Teil das numerische Integral über die Einzelbilder aufgetragen, was den Eindruck aus den Bildern bestätigt, auch wenn hier das Rauschen verhältnismäßig hoch ist. Die Inkubation ist insgesamt nicht so ausgeprägt wie bei reinem PMMA, wo während er ersten Pulse fast gar kein Leuchten zu sehen ist. Der Brennfleck verändert sich von einem abgerundeten Fleck zu einer sehr genau definierten sehr rechteckigen Fläche. Die Ablation ist definierter und die Abtragskrater zeigen zwar leicht abgerundete Kanten, aber im Gegensatz zum reinen PMMA eine genau definierte Abtragstiefe. 
Dazu passen die Depositionsraten während der ersten 100 Pulse, die in Abbildung 5.16 aufgetragen sind. Es ist zu sehen, dass sich der Verlauf der Depositionsraten von dem bei

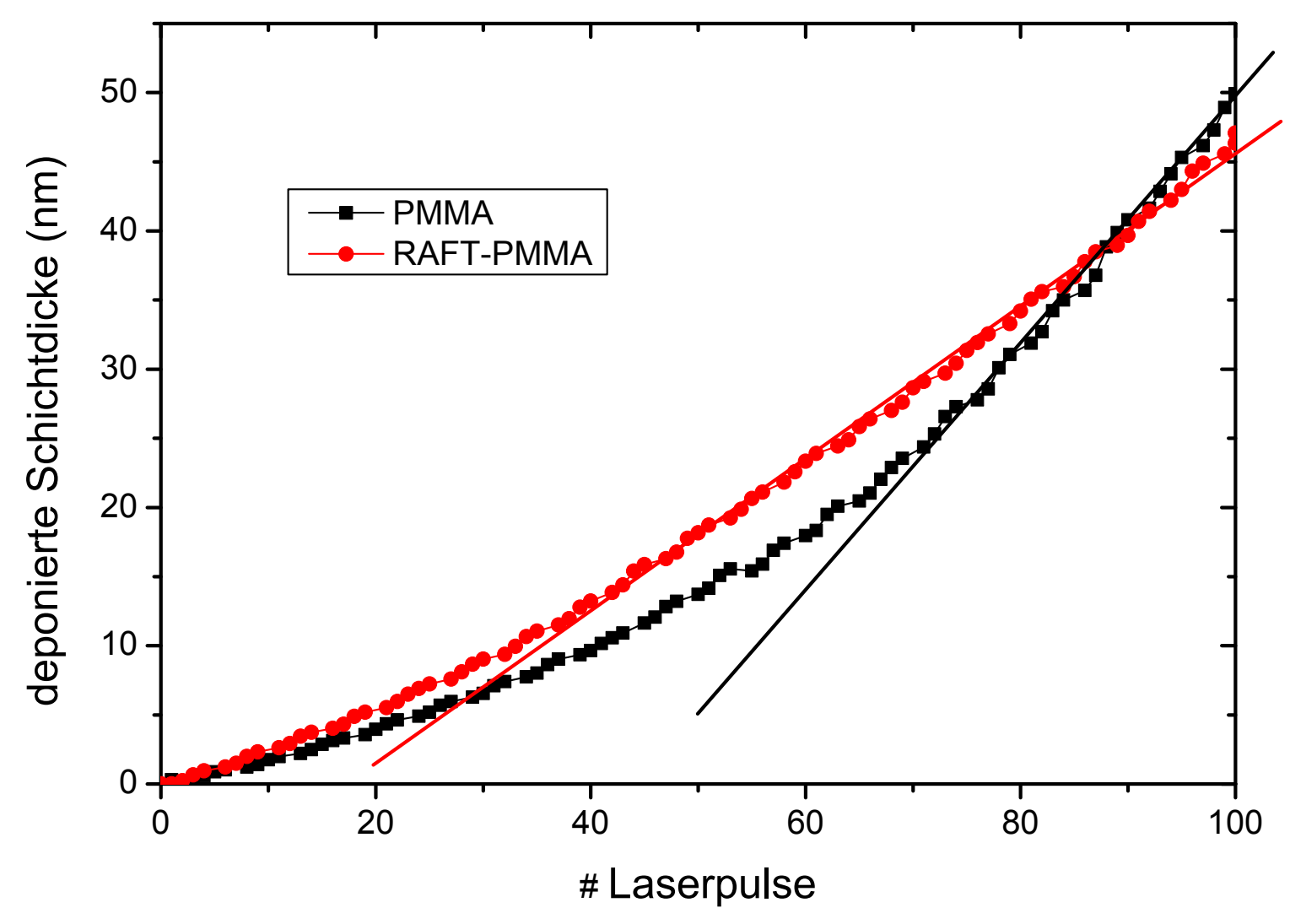

Abbildung 5.16: Deponierte Dicke bei der Deposition der ersten 100 Pulse von RAFTPMMA

Deposition von reinen PMMA-Targets unterscheidet. Zwar ist auch hier ein Bereich zu erkennen, in dem Inkubation stattfindet, jedoch ist dieser mit nur etwa 30-40 Pulsen etwa halb so lang. Auch wenn sich die Messungen durch leichte Abweichungen in der Depositionsgeometrie unterscheiden können, ist diese Veränderung signifikant. Die Einbringung der Absorber hat also einen Effekt in der verkürzten Inkubationsphase, jedoch ist diese nicht völlig aufgehoben. Damit ist zu vermuten, dass die Erhöhung der Absorption nicht ausreicht, um das Produkt grundsätzlich zu ändern.

Stellt man Filme mit diesem Ausgangsmaterial unter Standardbedingungen her, so erhält man Proben, die sich optisch nicht von den Standard-PMMA Proben unterscheiden lassen. Von der roten Färbung der Targets ist in den Filmen nichts zu sehen. Auch die Infrarotspektren, die in Abb. $5.17 \mathrm{zu}$ sehen sind, zeigen fast keinen signifikanten Unterschied 


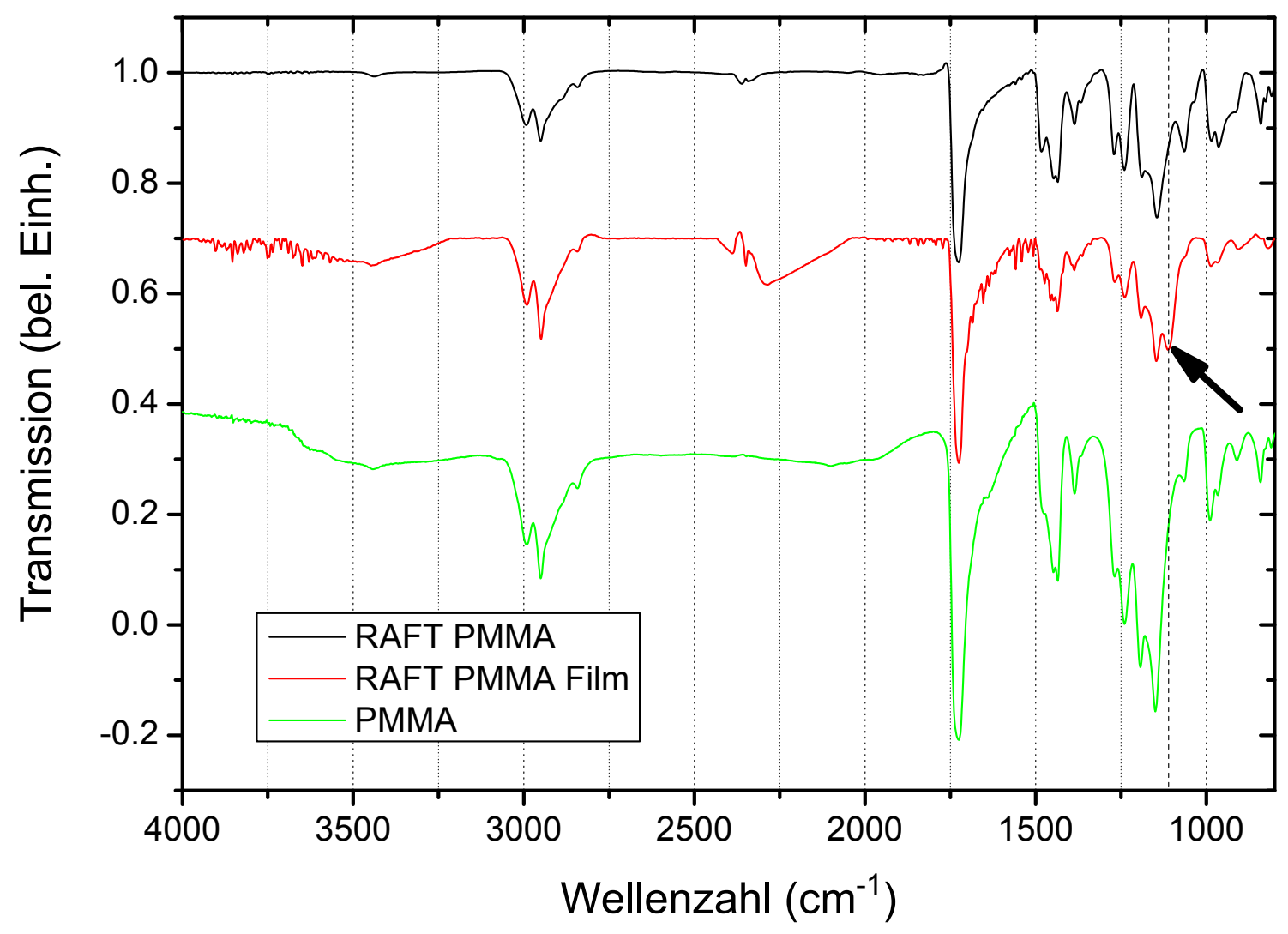

Abbildung 5.17: Infrarotspektrum von Filmen aus RAFT-polymerisiertem PMMA. Zum Vergleich ist ein Spektrum eines PMMA Films gezeigt. Die Kurven wurden für bessere Übersichtlichkeit gegeneinander verschoben.

zu den aus normalen PMMA-Targets deponierten Proben. Es taucht nur eine zusätzliche Bande bei $1108 \mathrm{~cm}^{-1}$ auf (Pfeil in der Abbildung), die weder in reinem PMMA noch in dem RAFT-polymerisierten Ausgangsmaterial zu finden ist. Diese Bande konnte nicht eindeutig zugeordnet werden. Weitere Unterschiede sind wiederum im Wesentlichen auf die Grundlinienkorrektur der Spektrometersoftware zurückzuführen. Es wird also auch hier PMMA übertragen.

Die Oberflächenstruktur der Filme (Abb. 5.18, links und Mitte), die aus den RAFTpolymerisierten Targets mit 300 Laserpulsen hergestellt wurden, zeigt einen deutlichen Unterschied zu den Filmen aus reinen PMMA-Targets (vgl. Abb. 5.9 und 5.10). Die Filme zeigen eine höhere Anzahl an Droplets, und es gibt eine Anzahl von sehr großen Droplets, ca. $20 \mu \mathrm{m}$ groß, die bei reinem PMMA nicht auftreten. Um zu entscheiden, ob diese extragroßen Droplets während der Inkubationsphase auftreten, in der der Unterschied zwi- 

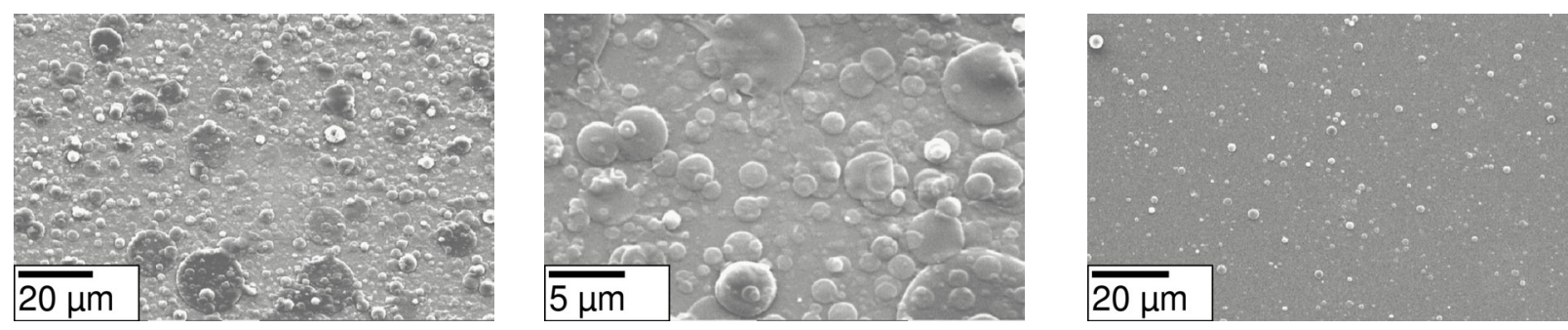

Abbildung 5.18: Filme aus RAFT-polymerisiertem PMMA. Links 300 Pulse, ca. 400fache Vergrößerung, Mitte 300 Pulse ca. 1600fache Vergrößerung und rechts 60 Pulse, ca. 400fache Vergrößerung.

schen reinem PMMA und dem hier verwendeten RAFT-polymerisierten PMMA besonders groß sein sollte, ist in der Abbildung 5.18 rechts eine Aufnahme von einem Film nach 60 Laserpulsen gezeigt. Hier zeigt sich, dass dieses Bild im Wesentlichen keine Unterschiede zu reinem PMMA aufweist. Das bedeutet, dass der Unterschied erst bei höherer Filmdicke auftritt und für niedrige Pulszahlen keine Rolle spielt.

Die höchsten Erwartungen an eine veränderte Struktur liegen in der Erhöhung der Kettenlänge im Filmmaterial. Dazu wurden SEC-Kurven gemessen, die in Abb. 5.19 aufgetragen sind. Zum Vergleich ist hier wieder die Kurve für reines PMMA gezeigt. Es ist zu sehen, dass sich die Kettenlänge gegenüber den aus handelsüblichem PMMA hergestellten Filmen nur unwesentlich verändert, sie steigt von ca. $M_{W}=8.000 \mathrm{~g} / \mathrm{mol}$ auf $9000 \mathrm{~g} / \mathrm{mol}$ $\left(M_{N}=6400 \mathrm{~g} / \mathrm{mol}\right)$. Diese Veränderung ist minimal.

Betrachtet man die Menge an Absorber, die dem Polymer zugefügt wurde, so ist diese im Vergleich zu den an den mit Antracen durchgeführten Untersuchungen sehr gering $(<1$ aromatischer Ring pro Monomereinheit). Dies führt vermutlich dazu, dass der grundsätzliche Mechanismus im Vergleich zu reinem PMMA nicht verändert wird. Zusätzlich treten bei höheren Pulszahlen noch größere Droplets auf, die vermutlich auf eine sich schneller verändernde Targetoberfläche zurückzuführen sind. Die geringe Menge an Absorber wird zerstört und damit auch das Material in größerer Tiefe von der Laserstrahlung angegriffen. Da sich dort starke Absorptionszentren befinden wird das Material im Verhältnis zu reinem PMMA in größerer Tiefe aufgeheizt. Die entstehenden Blasen haben bis zum Platzen länger Zeit, können größer werden und größere Droplets erzeugen. Das Ausgangsmaterial ist zusätzlich makroskopisch spröder als das reine PMMA, die Targets sind dadurch brüchiger und schwerer homogen herzustellen. Damit bringt diese Veränderung keine Vorteile für die Deposition von dünnen PMMA Filmen. 


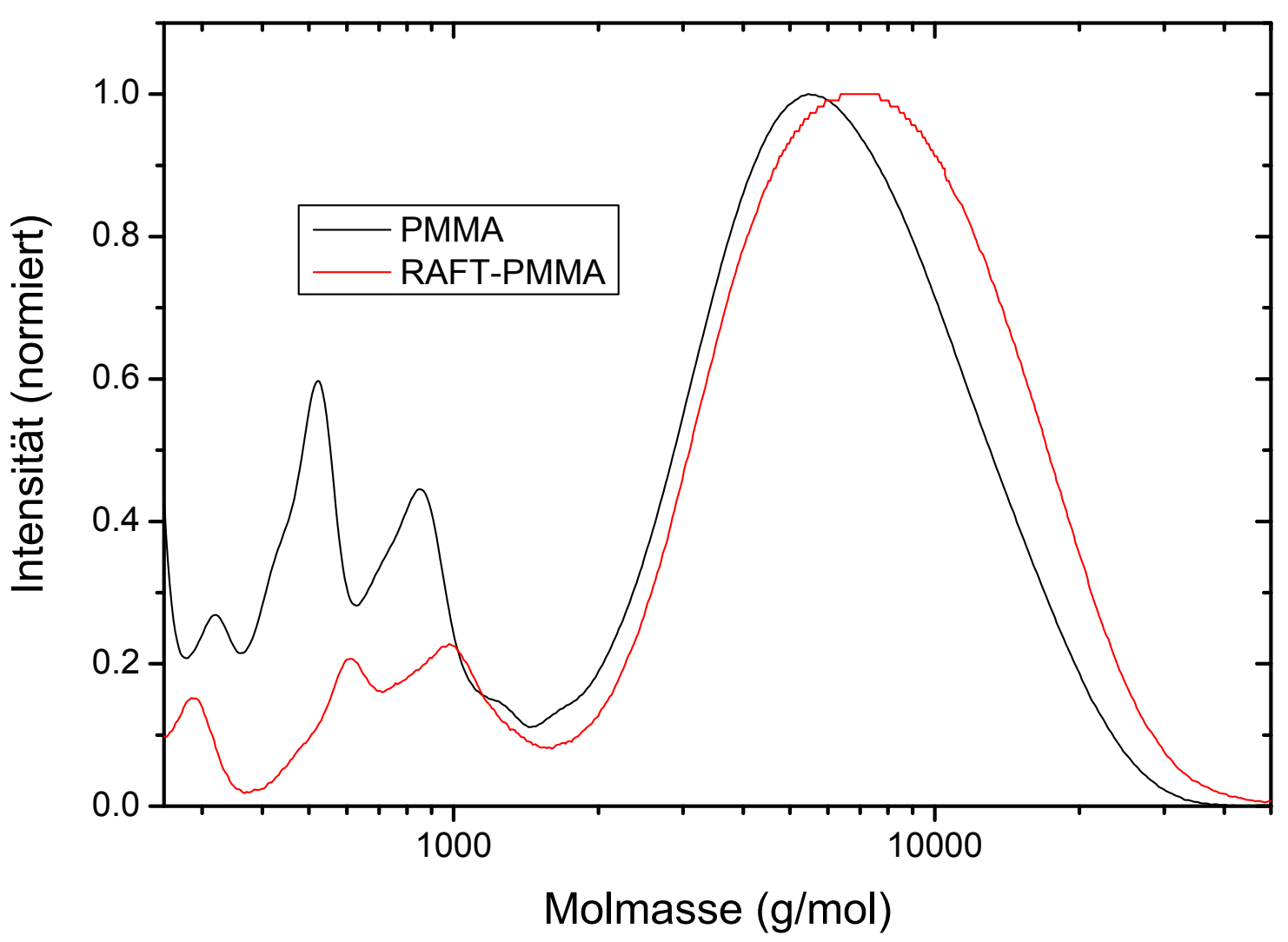

Abbildung 5.19: SE-Chromatogramme von Filmen aus RAFT-polymerisiertem PMMA

\subsection{Copolymere}

Ein Ansatz, die völlig homogene Verteilung des Absorbers zu realisieren und zusätzlich die Absorption weiter zu erhöhen, ist die Verwendung von Copolymeren aus MMA und einem stärker absorbierenden Material. Wie bereits in Abschnitt 4 gezeigt, sind Polystyrol-Filme durch gepulste Laserdeposition mit $248 \mathrm{~nm}$ Wellenlänge nicht herzustellen, da die aromatischen Ringe vollständig zerstört werden. Aus diesem Grund wurden Block-Copolymere aus MMA und Styrol mittels RAFT-Polymerisation hergestellt und als Targets verwendet. Die Strukturformel ist in Abb. 5.20 zu sehen.

Bilder der bestrahlten Targets während der ersten 100 Laserpulse sind in Abbildung 5.21 zusammengefasst. Es ist zu erkennen, dass der leuchtende Fleck erheblich größer ist als der für reines PMMA (Abb. 4.5) und das RAFT-polymerisierte PMMA (Abb. 5.15). Es sind sogar deutliche Anzeichen für eine Plasmabildung zu erkennen, wie sie beispielsweise für Metalle üblich ist. Dafür verändert sich in diesem Fall der Abtrag vom Target während der 


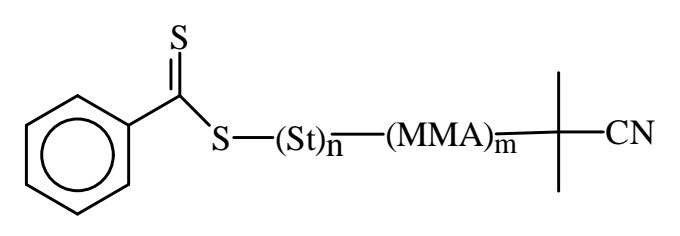

Abbildung 5.20: Strukturformel des mittels RAFT-Polymerisation hergestellten Copolymers

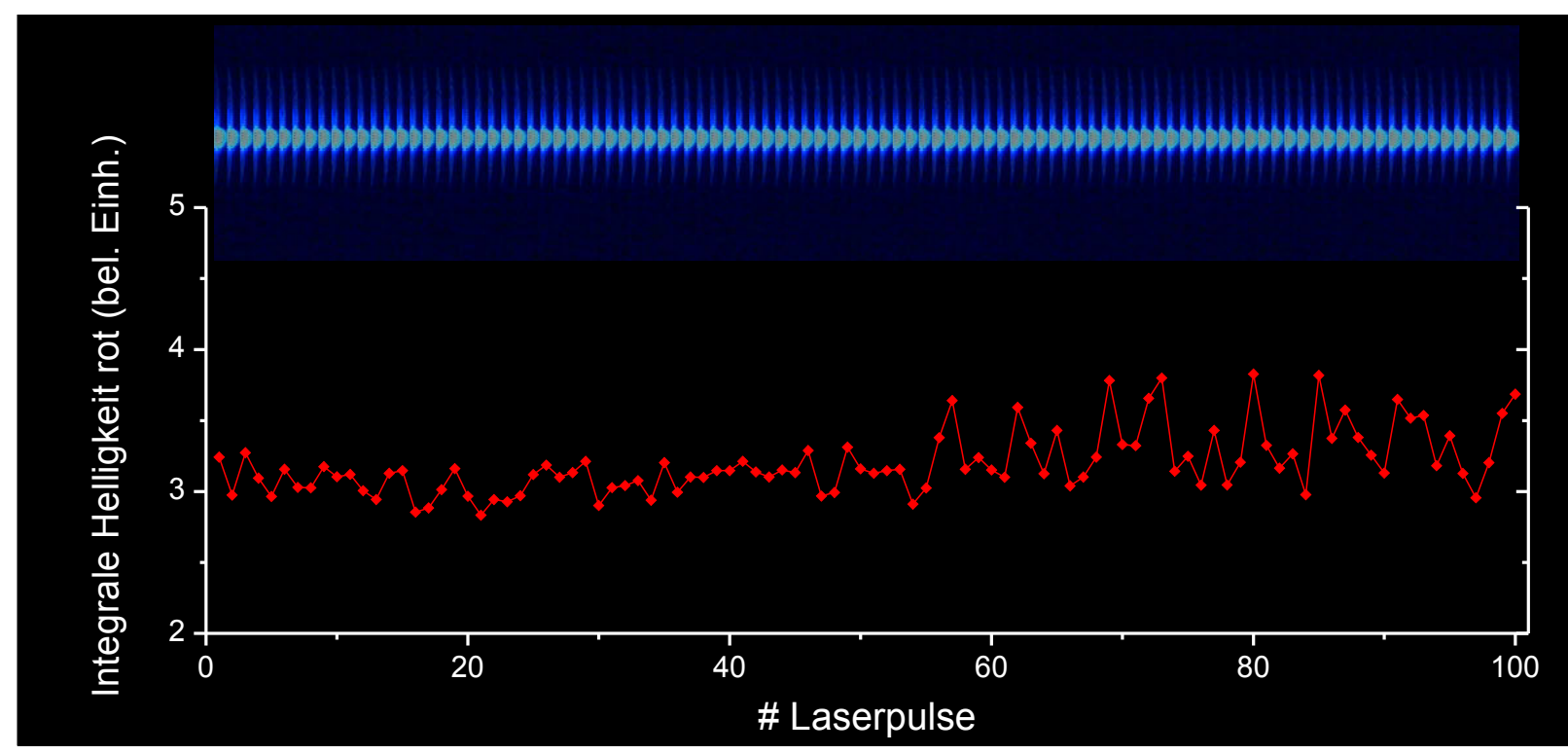

Abbildung 5.21: Oben: Entwicklung der Laserbestrahlung von RAFT-polymerisiertem Copolymer während der ersten 100 Laserpulse; Unten: Integral über die Helligkeit der Farbe Rot

ersten 100 Pulse nicht, abgesehen von dem Abtrag der Adsorbate beim ersten Puls. Dies lässt darauf schließen, dass das Ziel erreicht ist, den Ablationsprozess am Target über den Zeitraum des Abtrags stabil zu halten.

Betrachtet man die deponierte Schichtdicke von den Copolymertargets gegen die Pulszahl, so erhält man den Graphen 5.22. Zum Vergleich ist hier wieder die Rate von reinem PMMA aufgetragen. Es ist zu sehen, dass die Depositionsrate über die 100 Pulse konstant bleibt. Bei genauerem hinsehen erkennt man allerdings, dass die Rate im Verlauf der ersten 3-4 Pulse deutlich kleiner ist als für den Rest der Deposition. Auch wenn dies in den Kamerabildern nicht zu erkennen war, kann man sagen, dass ein geringer Effekt der Inkubation auftritt, wenngleich nur wenige Pulse lang. Auch in diesem Fall ist die Rate, wie schon bei der Zugabe von Antracen, sehr viel geringer als für reines PMMA. Beides spricht 


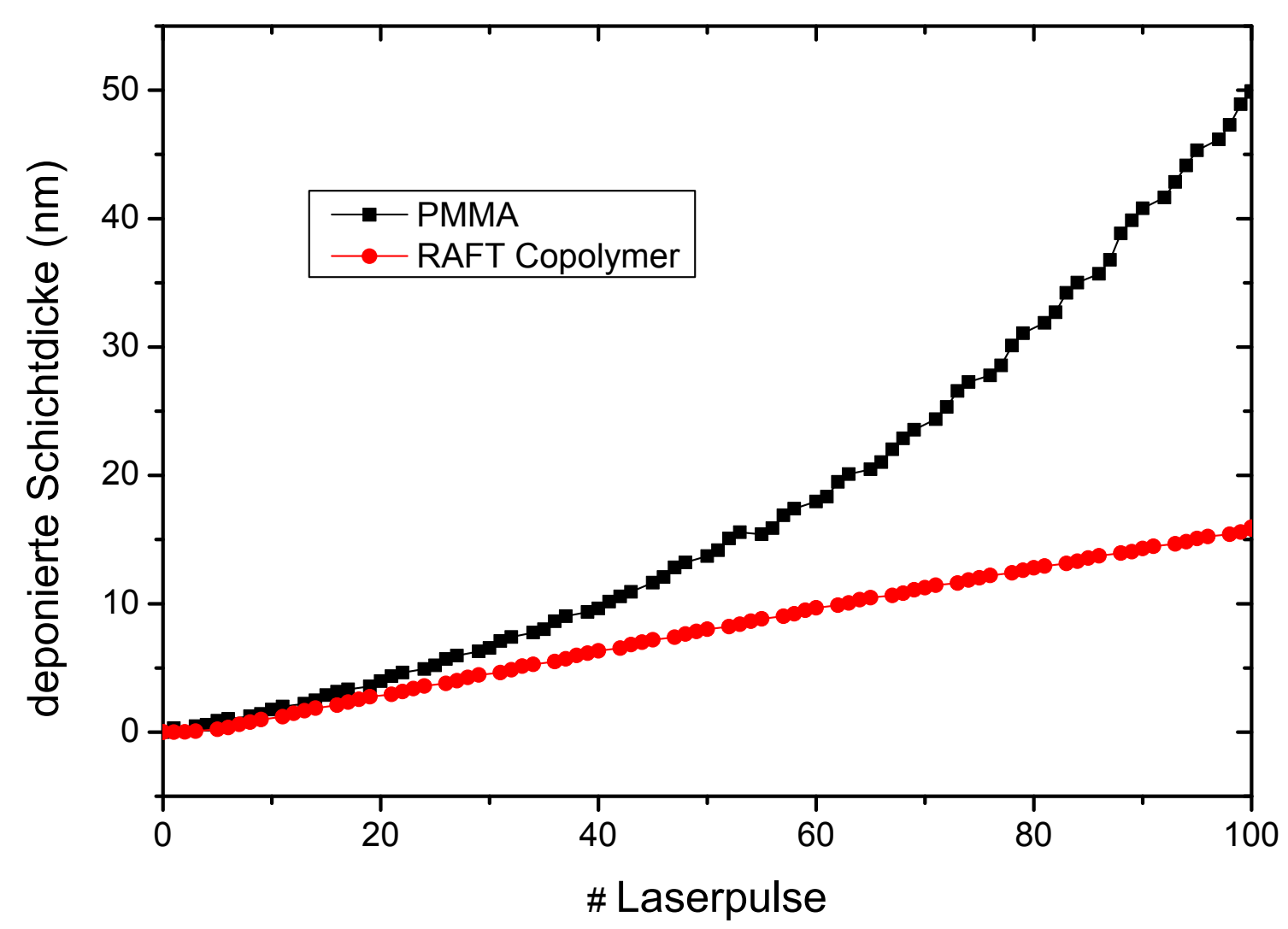

Abbildung 5.22: Deponierte Schichtdicke bei der Deposition der ersten 100 Pulse von RAFT-polymerisiertem Copolymer. Zum Vergleich ist die Rate für reines PMMA aufgetragen.

dafür, dass der Ablationsprozess deutlich stabilisiert werden konnte. Die Depositionsrate ist deutlich höher als durch die Beimengung von Antracen, was konsistent mit der deutlich vergrößerten Plasmabildung ist.

Bei dieser starken Veränderung der chemischen Zusammensetzung der Targets ist es interessant zu sehen, ob die Produkte eher das reine PMMA oder das Copolymer widerspiegeln. Zur Klärung dieser Frage wurden Infrarotspektren aufgenommen, die in Abbildung 5.23 gezeigt sind. Zusätzlich sind zum Vergleich die Spektren eines laserdeponierten PMMA Films und von Polystyrol, das durch auftropfen einer Lösung auf ein Substrat und darauffolgendes Abdampfen des Lösungsmittels (drop cast) präpariert wurde, aufgetragen. Es ist zu sehen, dass die Spektren sehr gut mit denen der laserdeponierten PMMA-Filme übereinstimmen. Keine der charakteristischen Banden, die den Bindungen im Polystyrol zugeordnet werden könnten, findet sich in dem Film, es ist also davon auszugehen, dass 


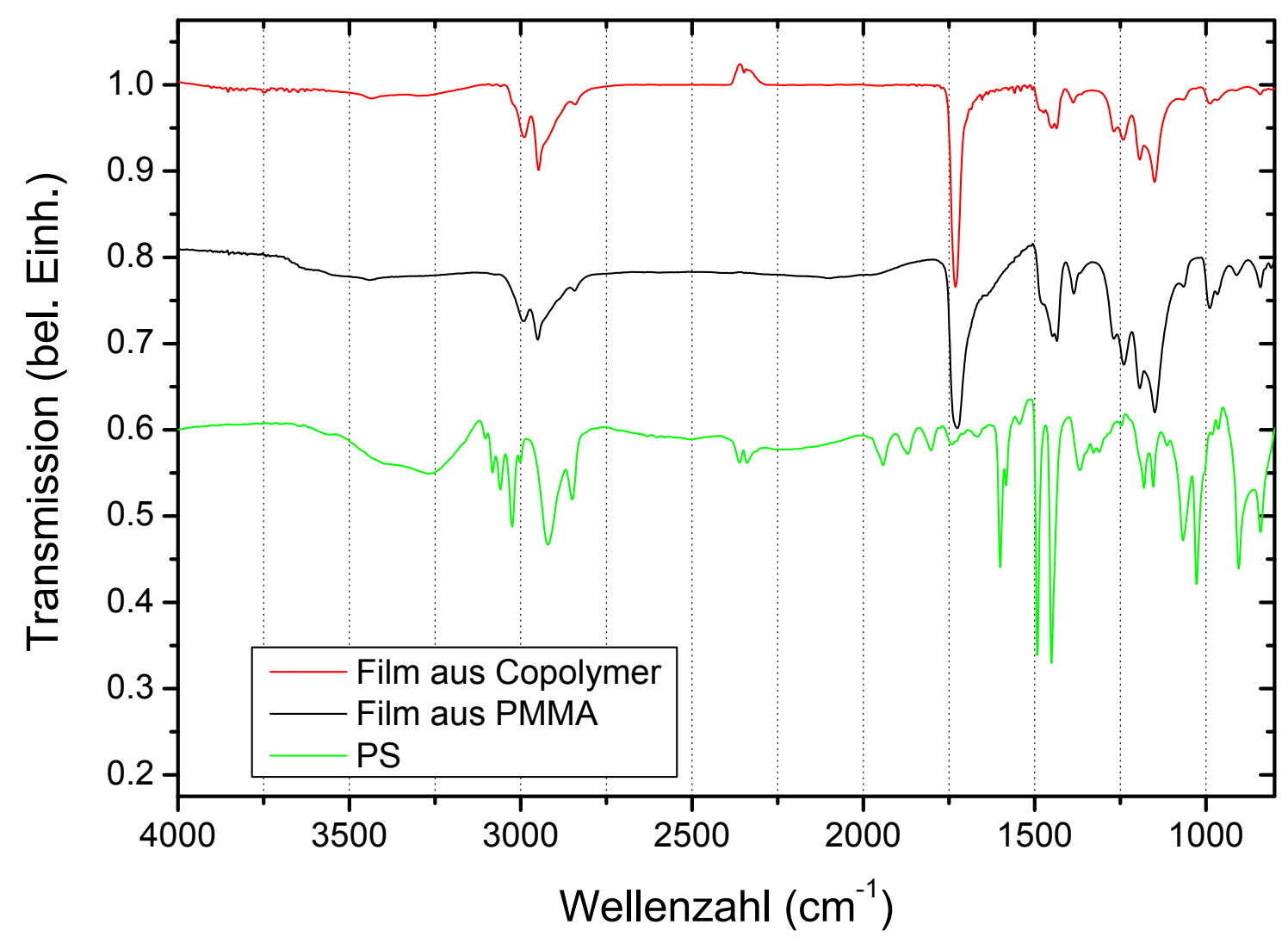

Abbildung 5.23: Infrarotspektrum von Filmen aus RAFT-polymerisiertem Copolymer. Zum Vergleich ist ein Spektrum eines Films aus reinem PMMA und von reinem PS gezeigt. Die Kurven wurden für bessere Übersichtlichkeit gegeneinander verschoben.

bei den hier verwendeten Laserfluenzen — bis zur Nachweisgrenze mit dieser Methode das gesamte Styrol zerstört und nur PMMA deponiert wurde. Dies war nach den Ergebnissen mit reinem Polystyrol in Kapitel 4 grundsätzlich zu erwarten, ob jedoch auch im Copolymer eine völlige Zerstörung eintritt war unklar. Weiterhin ist zu erkennen, dass die zusätzliche Bande bei $1108 \mathrm{~cm}^{-1}$, die für das RAFT-polymerisierte PMMA im Film aufgetreten war, nicht auftritt. Dies lässt darauf schließen, dass diese durch die Nachbarschaft des RAFT-Reagenz mit dem PMMA begründet war, die in dem Copolymer nicht vorhanden ist, zuerst Polystyrol folgt. Diese Ergebnisse, verbunden mit der Betrachtung der Plasmabildung und der Depositionsrate, sprechen dafür, dass die intensive Leuchterscheinung auf die Zerstörung und den Abtrag des Styrols zurückzuführen ist, die Ablation und Deposition von PMMA dagegen etwas verzögert eintritt. 
Rasterelektronenmikroskopbilder der mit 300 Pulsen aus Copolymertargets hergestellten Filmoberfläche sind in Abbildung 5.24 mit zwei verschiedenen Vergrößerungen gezeigt. Es ist zu sehen, dass sich die Oberfläche sowohl von reinem PMMA als auch von RAFT-
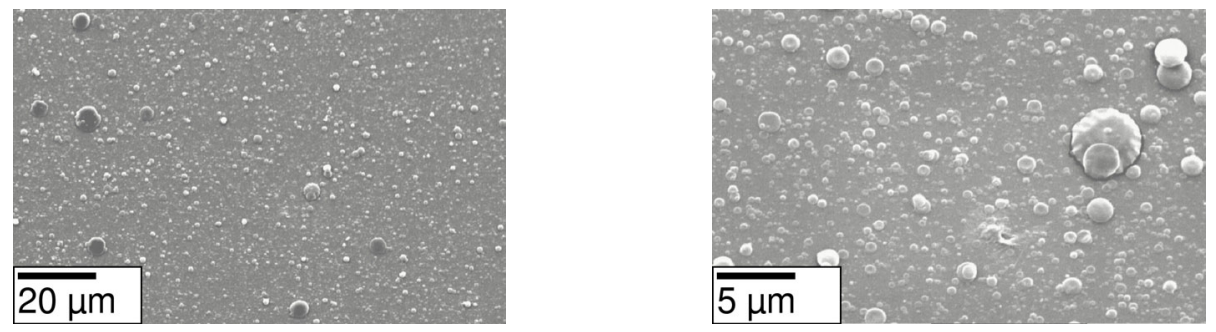

Abbildung 5.24: Filme aus RAFT-polymerisiertem Copolymer mit 300 Pulsen. Links ca. 400fache und rechts ca. 1600fache Vergrößerung

polymerisiertem PMMA deutlich unterscheidet. Sie ist zwar wiederum übersäht mit Droplets, jedoch liegen diese nicht mehr in der Größenordnung von 1-4 $\mu \mathrm{m}$. Statt dessen sind kaum Droplets über 1,5 $\mu \mathrm{m}$ Größe zu finden, jedoch sehr viele mit einer Größe von unter $800 \mathrm{~nm}$ bis hinunter zur Auflösungsgrenze des verwendeten Rasterelektronenmikroskops.

Aufgrund dieser Besonderheit wurden auch von den Filmen dieses Materials Rasterkraftmikroskopaufnahmen gemacht, um die Höhenskala zu ermitteln. Diese sind in Abbildung 5.25 gezeigt. Dort ist in der Übersichtsaufnahme (links) zu sehen, dass die Bilder mit
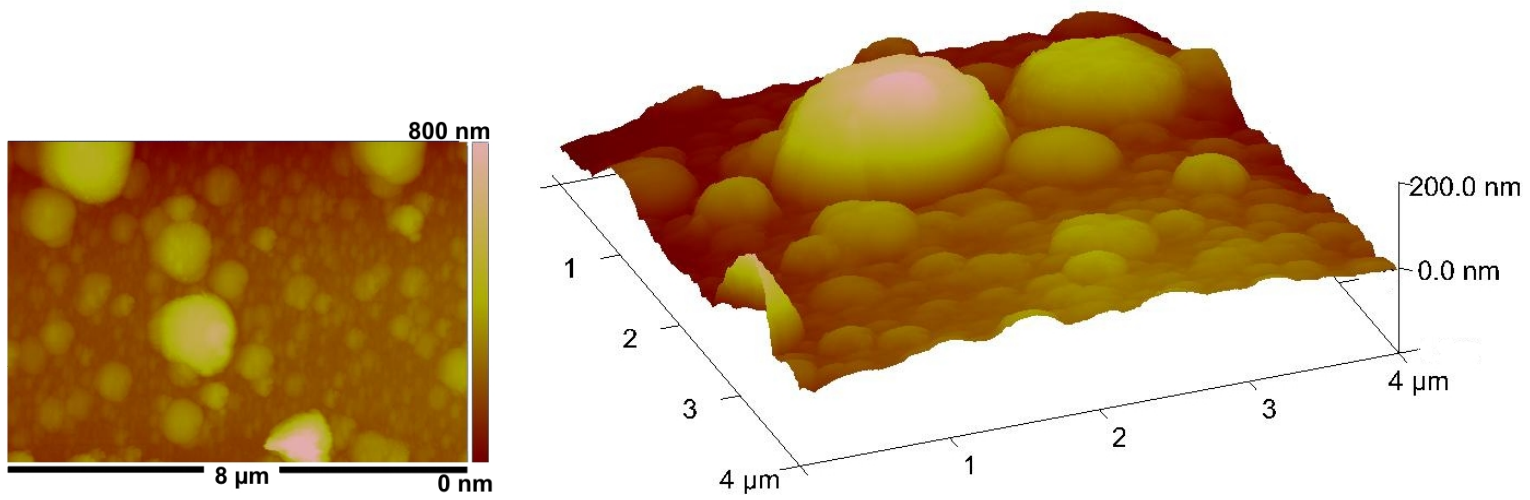

Abbildung 5.25: Rasterkraftmikroskopbilder von Filmen aus RAFT-polymerisiertem Copolymer. Es sind zwei verschiedene Vergrößerungen gezeigt, das rechte Bild ist überhöht dargestellt.

denen der Probe für das Rasterelektronenmikroskop vergleichbar sind. Die Droplets sind maximal ca. $200 \mathrm{~nm}$ hoch und damit deutlich flacher als es Halbkugeln wären. Die Struktur sieht damit insgesamt ähnlich aus wie die von reinen PMMA-Targets hergestellten, nur die Größenskala der Droplets ist um einen Faktor 3-4 kleiner. 
In Abb. 5.26 ist das Chromatogramm für die Filme aus Copolymertargets zu sehen. Zum

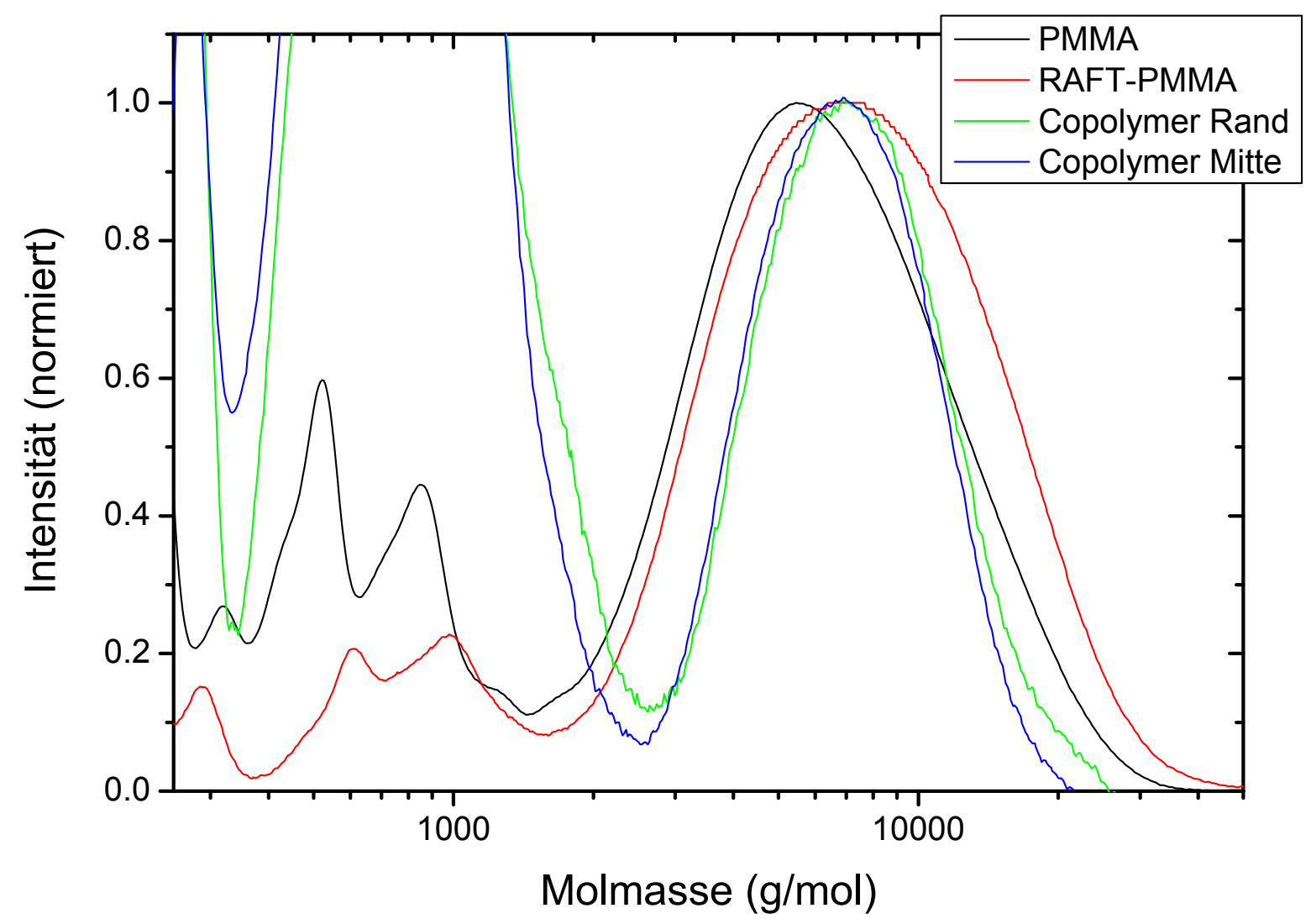

Abbildung 5.26: SE-Chromatographie von Filmen aus RAFT-polymerisiertem Copolymer

Vergleich wurden Kurven für Filme aus reinen PMMA Targets und RAFT-polymerisierten Targets mit eingetragen. Es zeigt sich, dass hier die Kettenlänge nur minimal gegenüber der der Filme aus reinen PMMA-Targets erhöht ist. Sie ist identisch mit dem RAFTpolymerisierten Polymer bei $M_{W}=9.000 \mathrm{~g} / \mathrm{mol}$, nur die Verteilung der Kettenlängen ist schärfer, das Zahlenmittel steigt damit auf $M_{N}=6.500 \mathrm{~g} / \mathrm{mol}$.

Es ist also festzustellen, dass die Ablation durch die Verwendung von Copolymeren deutlich verändert wird. Vor allem die Morphologie der Filmoberfläche verändert sich deutlich und die Droplets werden weniger dominant. Gleichzeitig ist die Depositionsrate hoch und die Targetalterung ist deutlich schwächer als für reine PMMA-Targets. Allerdings ist das andere Ziel, eine chemisch möglichst wenig veränderte Struktur zu erhalten, die lange Polymerketten aufweist, nicht erreicht. Wie durch die geringen Unterschiede in der Depositionsrate während der ersten Pulse geschlossen werden kann, tritt auch hier Inkubation 
auf. Allerdings ist diese wohl weniger stochastisch, sondern tritt erzwungen im Bereich der Styrol-Ketten und dadurch gleichmäßiger verteilt auf.

\subsection{MAPLE}

Die Targetmodifikationen der letzten Abschnitte werden durch die in Abschnitt 2.1.3 schon vorgestellte MAPLE (Matrix Assisted Pulsed Laser Evaporation) [87, 138] Methode konsequent maximiert. Für diese Variation der PLD ist das zu deponierende Material gegenüber dem Absorber in der Minderheit und in einer Absorbermatrix eingebettet. Dies wird realisiert, indem ein UV absorbierendes Lösungsmittel verwendet wird. In diesem ist das Material bei guter Löslichkeit homogen verteilt. Die Lösung wird durch flüssigen Stickstoff schockgefroren und als Targetmaterial verwendet. Für MAPLE wird berichtet, dass sich verschiedene Materialien von Polymeren [106] über Kohlenstoffnanoröhrchen [138] und metallorganische Sterncopolymere [37] bis zu Proteinen mit intakter biologischer Funktion [90] deponieren lassen, auch wenn die Berichte bezüglich Schichtdickenhomogenität, Rauigkeit und teilweise chemischer Unversehrtheit vielfach unbefriedigend sind.

\subsubsection{Dimethoxyethan als Matrix}

Das für MAPLE häufig verwendete Lösungsmittel Wasser [15, 16] scheidet in diesem Fall aus, da die meisten Polymere darin nicht löslich sind. Ein weiteres in der Literatur verwendetes Lösungsmittel für die Matrix ist Dimethoxyethan (DME) [37], auch bekannt unter dem Namen Ethylenglykoldimethylether oder Dimethylglykol (Strukturformel Abb. 5.27). Es wurden Lösungen mit 2 Gew.\% PMMA verwendet, die über mindestens 20 Stunden homogenisiert wurden.
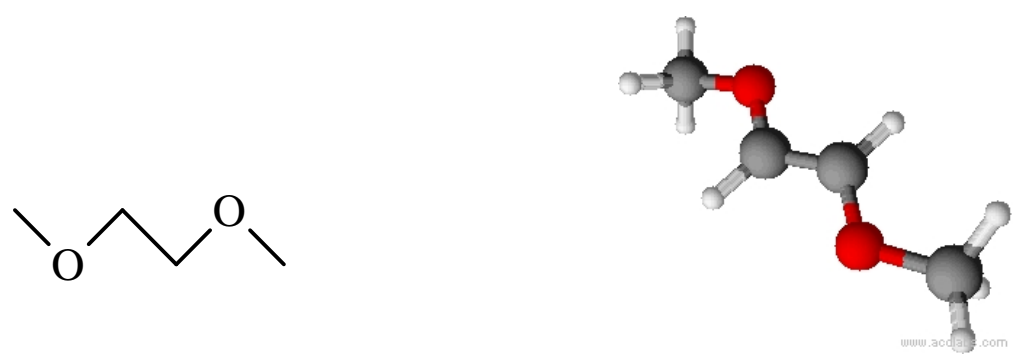

Abbildung 5.27: Strukturformel von Dimethoxyethan (DME)

Filme, die mit dieser stark veränderten Methode hergestellt wurden, zeigen schon makroskopisch einen deutlichen Unterschied zu den unter Standardbedingungen laserdepo- 
nierten PMMA-Filmen. Sie sind bis auf Schichtdickeninterferenzfarben farblos transparent, zeigen eine verhältnismäßig schlechte Substrathaftung und lassen sich leicht abkratzen. Des Weiteren sind sie in üblichen Lösungsmitteln wie THF fast rückstandsfrei abzulösen.

Dass die Filme nicht glatt sind, ist schon im Lichtmikroskop ersichtlich. Bei etwa 50fa-
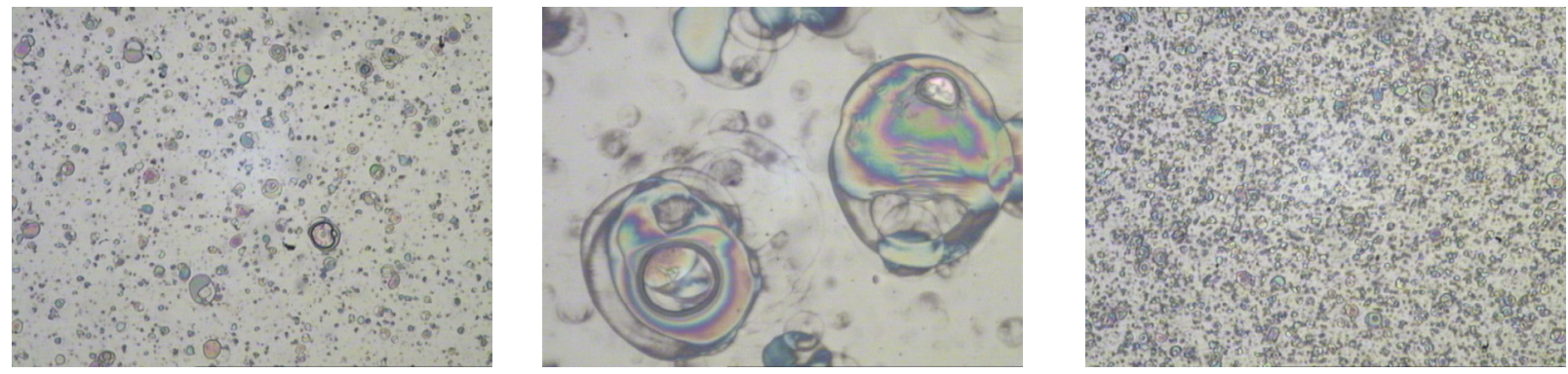

Abbildung 5.28: Lichtmikroskopaufnahmen von PMMA Filmen mittels MAPLE mit DME hergestellt. Links: 500 Pulse, ca. 50fache Vergrößerung; mitte: 500fache Vergrößerung; rechts: 5000 Pulse, ca. 50fache Vergrößerung

cher Vergrößerung (Abb. 5.28 links und rechts) ist zu sehen, dass der Film aus einer Vielzahl von Droplets besteht, deren Anzahl mit zunehmender Pulszahl steigt. Bei großer Vergrößerung (Mitte) ist zu sehen, dass diese Droplets keineswegs rund sind, wie es sonst für Droplets auf PMMA-Filmen bekannt ist, sondern teilweise sehr komplizierte Strukturen ausbilden.

Erhöht man jetzt Auflösung und Schärfentiefe durch die Verwendung eines Elektronenmikroskops (Abb.5.29), ist zu sehen, dass die Droplets sehr flach auf dem Substrat aufliegen und überhaupt nicht mit üblichen Droplets zu vergleichen sind. Es erweckt eher den Ein-
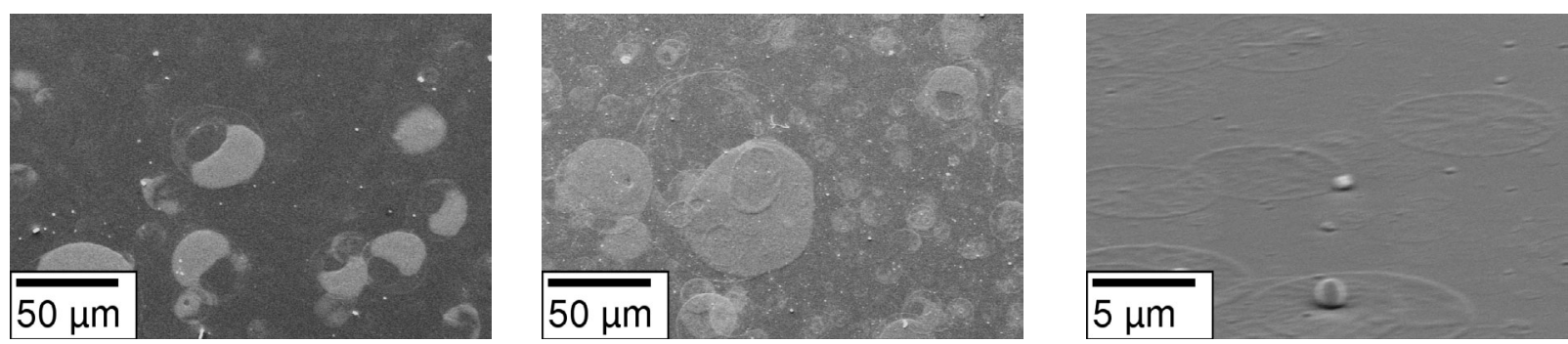

Abbildung 5.29: Rasterelektronenmikroskopaufnahmen von PMMA Filmen mittels MAPLE mit DME hergestellt. Links: 500 Pulse, ca. 200fache Vergrößerung; mitte: 5000 Pulse, ca. 200fache Vergrößerung; rechts: 5000 Pulse, ca. 2.000fache Vergrößerung, Probe für die Aufnahme um $63^{\circ}$ gekippt

druck, dass es sich um die Überreste flüssiger Tropfen von im Lösungsmittel gelösten Polymer gehandelt hat, die auf der Oberfläche liegen blieben und sich unter Zurücklassung von Trockenringen von PMMA verflüchtigten. Kippt man die Probe im Rasterelektronenmikroskop (Abb. 5.29 rechts) sieht man in vielen Trockenflecken ein annähernd kugelförmiges 
Gebilde, meistens in der Nähe des Zentrums. Hierbei könnte es sich um PMMA handeln, das bereits während des Fluges eingetrocknet und ausgefallen ist und sich dann in einem Tropfen aus gesättigter Lösung auf das Substrat gesetzt hat. Der Rest des Lösungsmittels ist dann auf dem Substrat wie üblich getrocknet und der fast kugelförmige Anteil unverändert geblieben. Hier ist zu beachten, dass die Targets mit etwa $-180{ }^{\circ} \mathrm{C}$ sehr kalt sind, Mercado et al. verwenden beispielsweise nur $-120{ }^{\circ} \mathrm{C}$ kalte Targets für $\mathrm{CHCl}_{3}$ [77, Toftmann et al. nur $-50{ }^{\circ} \mathrm{C}$ für Wasser [94, 125], was hier apparativ nicht möglich ist. Die Tröpfchen werden hier vermutlich kälter abgelöst und benötigen während des Fluges mehr Zeit, um völlig zu verdampfen. Allerdings sind auch die Berichte in der Literatur über glatte Filme nicht auf mikroskopischer Skala gezeigt. Auf dem Substrat verdampft das Lösungsmittel dann vermutich sehr schnell.

Betrachtet man die chemische Zusammensetzung mittels Infrarotspektroskopie (siehe Abbildung 5.30, so erkennt man nur leichte Veränderungen gegenüber den Spektren für

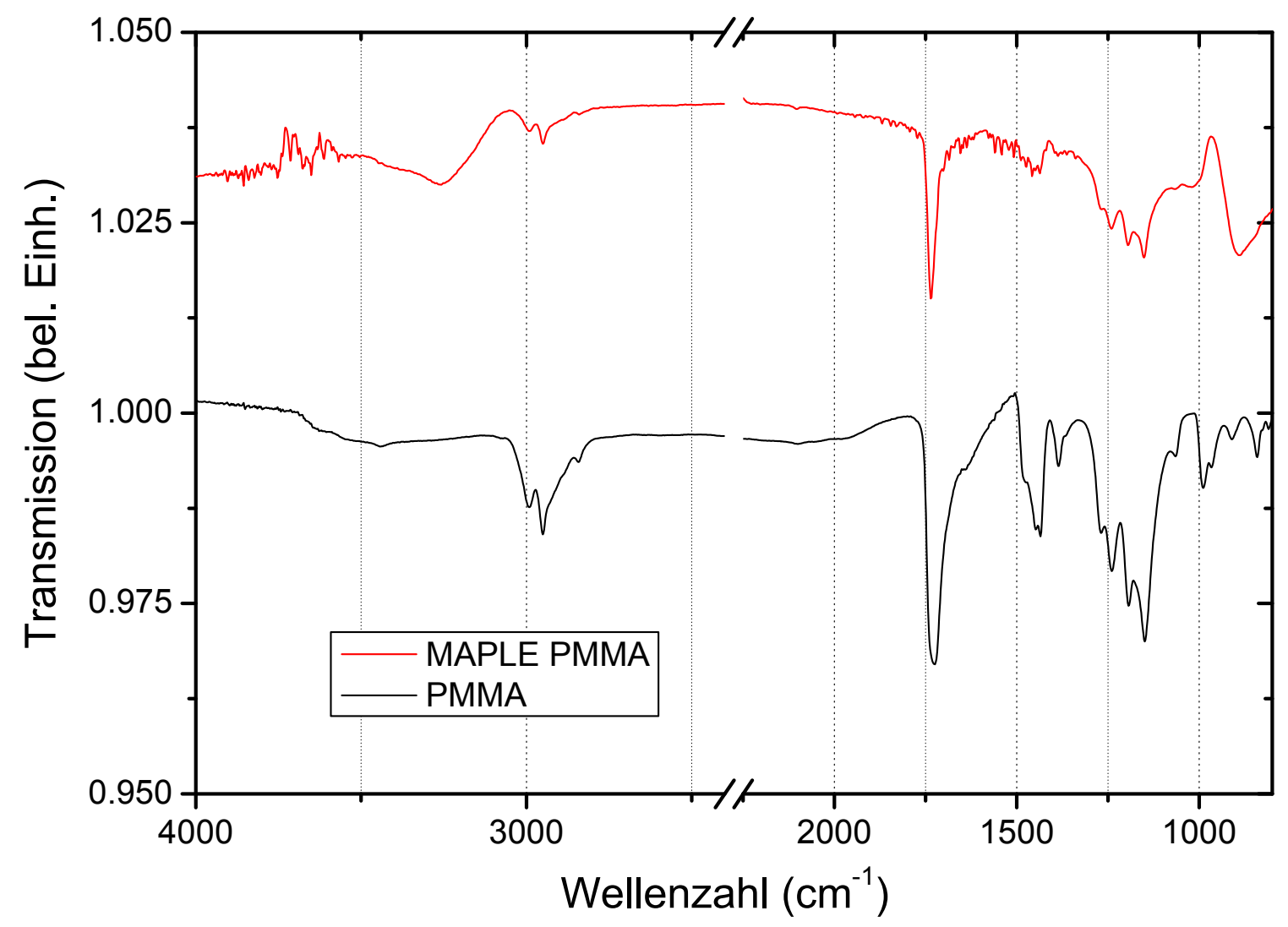

Abbildung 5.30: Infrarotspektren von MAPLE PMMA mit DME und von üblichem laserdeponiertem PMMA. Die Spektren wurden gegeneinander verschoben. 
Filme aus festen PMMA-Targets. In diesem Fall bleibt es unklar, ob die Unterschiede durch die schlechte Auflösung bei der geringen Schichtdicke stammen, oder ob tatsächlich chemische Veränderungen auftreten. Diese betreffen im Wesentlichen das starke Zurückgehen der Deformationsbanden der $\mathrm{O}-\mathrm{CH}_{3}, \mathrm{CH}_{2}$ und $\alpha \mathrm{CH}_{3}$ Bindungen, die im Bereich zwischen 1438$1483 \mathrm{~cm}^{-1}$ liegen. Hierbei handelt es sich um mehrere verschiedene Gruppen an deutlich unterschiedlichen Stellen im Monomer. Damit ist es deutlich wahrscheinlicher, dass diese in der zu geringen Auflösung untergehen, als dass das Polymer soweit zerstört ist, gleichzeitig aber die anderen Banden zeigt, ohne dass zusätzliche Banden auftreten.

Da in der Literatur teilweise berichtet wird, dass mittels MAPLE selbst sehr komplizierte Moleküle weitgehend zerstörungsfrei deponiert werden können [37], ist der interessanteste Punkt die Untersuchung des Molekulargewichts der Produkte. Diese ist in Abbildung 5.31 gezeigt. Wie sich herausstellt, ist das Bild völlig anders als für unter

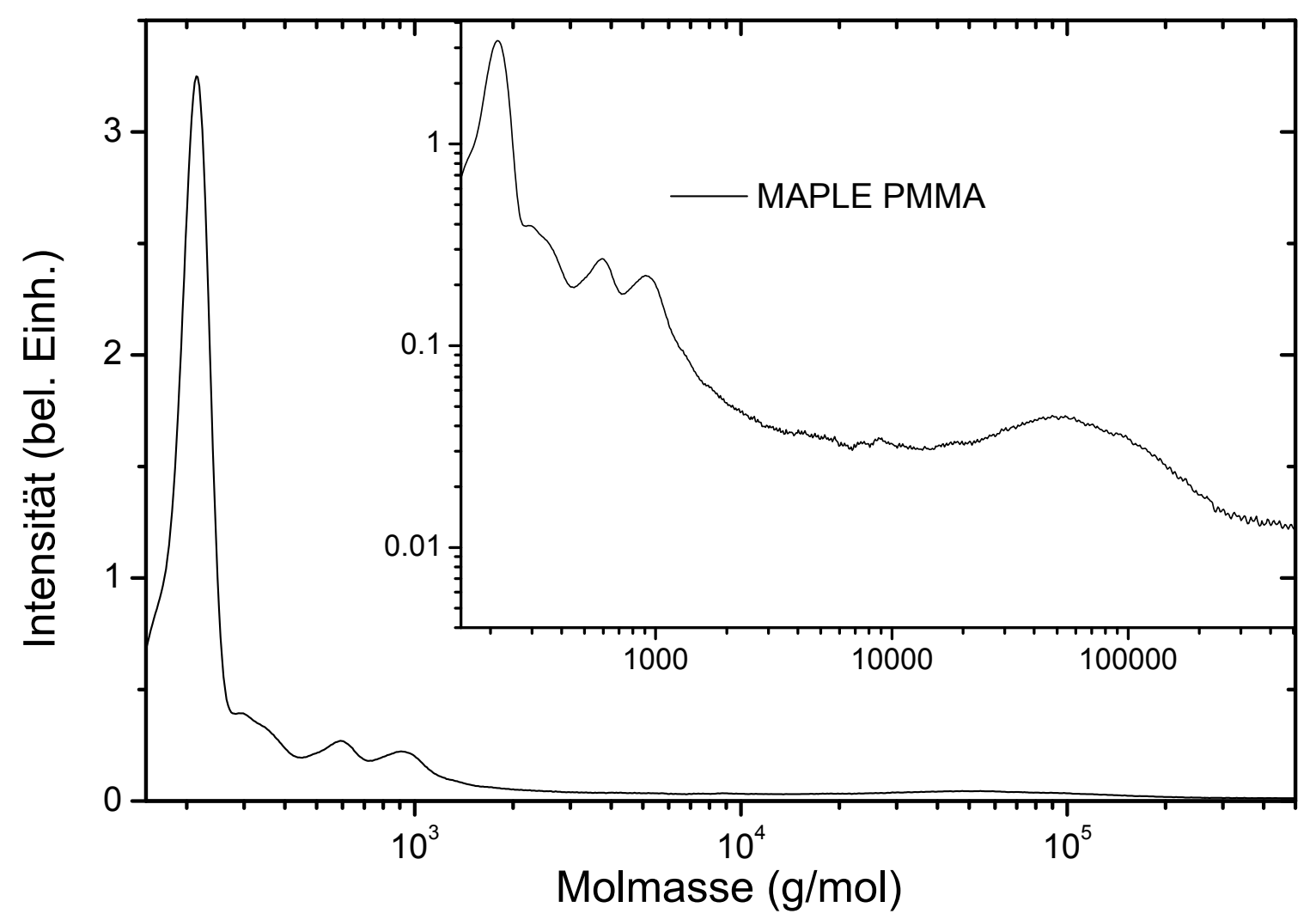

Abbildung 5.31: SE-Chromatogramme von Filmen aus MAPLE PMMA mit DME

Standardbedingungen laserdeponierte PMMA-Filme. Es dominiert ein deutlicher Anteil an Oligomeren, vielfach kleiner als für reines PMMA. Vor allem das Dimer sticht hervor. 
Nur bei genauem Hinsehen, fällt ein kleiner Peak mit mittlerer Masse $M_{W}=73 \mathrm{~kg} / \mathrm{mol}$ $\left(M_{N}=56 \mathrm{~kg} / \mathrm{mol}\right)$ auf. Um diesen deutlicher zu machen ist im Inset von Abbildung 5.31 noch einmal die gleiche Messung logarithmisch dargestellt. Ob der Peak tatsächlich signifikant ist, oder aus dem Rauschen der chromatographischen Säulen kommt ist nicht $100 \%$ ig sicher, wenn man ihn als relevant ansieht, erhält man dank der großen Breite einen Anteil von etwa $81 \%$ an diesen verhältnismäßig sehr langkettigen Molekülen. Da Dimere von MMA nicht fest und leicht flüchtig sind, ist es wohl auszuschließen, dass der Film im wesentlichen daraus besteht. Auch eine Art Matrix fällt aus, da das Material vollständig für die SEC aufgelöst wird. Damit wird unterstützt, dass der langkettige Anteil tatsächlich real ist. Der Anteil ist niedriger als für Filme, die aus reinem PMMA hergestellt wurden (90-95\%) und die Verteilung ist sehr breit. Von zerstörungsfreier Ablation kann keine Rede sein, auch wenn das Ergebnis deutlich verändert ist.

\subsubsection{DME-Matrix unter Inertgasatmosphäre}

Eine Idee zur Vermeidung der Deposition flüssiger Tröpfchen von Polymerlösung ist die Veränderung des Target-Substrat-Abstands. Dies ergibt im experimentell zugänglichen Bereich keine messbaren Veränderungen. Eine weitere Möglichkeit das Verdampfen des Lösungsmittels schon während des Fluges zu verbessern sollte die Verwendung eines inerten Hintergrundgases sein. Dadurch sollte durch eine vermehrte Wechselwirkung in der Flugphase das Lösungsmittel schneller verdampfen und so dieser Prozess nicht erst auf dem Substrat stattfinden. Es ist also ein Druck zu wählen, der eine recht starke Wechselwirkung erzeugt.

Da bei den hier verwendeten Targettemperaturen das üblichste Gas Ar (z. B. [37]) bereits flüssig wird, wurde stattdessen Ne verwendet. Abschätzungen für die Gasdrücke, die nötig sind, um den Prozess der gepulsten Laserdeposition zu verändern, kann man aus früheren Arbeiten zum Vergleich der Hintergrundgase [101, 100] gewinnen; auch der Vergleich mit entsprechenden Ar-Gasdrücken ist damit möglich. Es wurde ein Druck von 0,25 mbar Ne verwendet, der in der Wechselwirkung einem Ar-Druck von etwa 0,1 mbar entsprechen und eine starke Beeinflussung bewirken sollte. Für zusätzliche Vergleichsmes-

sungen wurde ein Hintergrundgasdruck von 0,4 mbar verwendet, was keine weiteren Effekte zeigte.

Betrachtet man die Rasterelektronenmikroskopischen Bilder von Filmen, die unter einem Druck von 0,25 mbar Ne hergestellt wurden (Abb. 5.32), so sieht man, dass der Prozess im Vergleich zur Deposition im UHV höchstens geringfügig verändert wurde. Es 

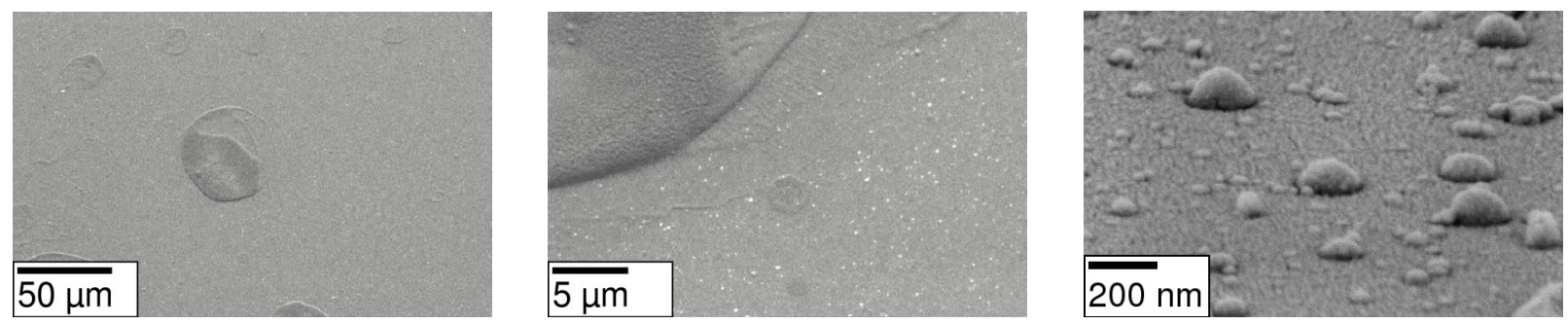

Abbildung 5.32: Rasterelektronenmikroskopaufnahmen von PMMA Filmen mittels MAPLE mit DME unter 0,25 mbar Neon-Atmosphäre mit jeweils 1000 Pulsen hergestellt. Links: ca. 200fache Vergrößerung; Mitte: ca. 1600fache Vergrößerung; rechts ca. 40.000fache Vergrößerung, Probe um $75^{\circ}$ gekippt.

sind weiter die großen, flachen Tropfen zu sehen, die an Trockenflecken erinnern. Bei hoher Vergrößerung sind zusätzlich eine Vielzahl sehr kleiner Punkte zu erkennen. Diese Bilder, vor allem die kleinen Punkte, sind vergleichbar mit den von Mercado et al. [77] für MAPLEdeponiertes PLGA (Poly (lactid-co-glycolsäure)) gezeigten, wobei dort als Lösungsmittel $\mathrm{HCCl}_{3}$ und als Hintergrundgas 0,13 mbar Ar verwendet wurde. Die Autoren führen die kleinen Droplets darauf zurück, dass das Lösungsmittel erst auf dem Substrat verdampft. Die relativ steilen Flanken, erkennbar im rechten Bild, für das die Probe zum Detektor hin verkippt wurde, lassen jedoch vermuten, dass die feinen Tröpfchen schon im Flug entstanden sind. Vergleicht man die Bilder mit denen der Proben für die kein Hintergrundgas verwendet wurde, so sind die kleinen Droplets der wesentliche Unterschied. Es scheint so, als wäre während des Fluges mehr Lösungsmittel verdampft, weshalb mehr kleine, hohe Tröpfchen entstanden sind. Zusätzlich sind sie feiner verteilt. Dies zeigt, dass tatsächlich ein Druckregime erreicht wurde, das einen starken Einfluss auf die Deposition ausübt.

Als wesentlicher Unterschied ist eine stark verringerte Depositionsrate auffällig. Da bei einem Hintergrundgasdruck von 0,4 mbar Ne keine weitere Veränderung auftritt und die Herstellung unter so hohen Gasdrücken einen erheblichen experimentellen Aufwand darstellt, wurde der Einfluss der Inertgasatmosphäre an dieser Stelle nicht weiter untersucht.

\subsubsection{Toluol als Matrix}

Da Chromophore für Strahlung mit $248 \mathrm{~nm}$ Wellenlänge hauptsächlich durch $\pi$-Elektronensysteme bestimmt werden und vor allem aromatische Ringe geeignet sind, liegt die Verwendung eines weiteren Lösungsmittels nahe, das zu diesem Zweck in der Literatur

erst in allerletzter Zeit betrachtet wurde, Toluol [106] (Strukturformel siehe Abb. 5.33). Es schmilzt bei $-93{ }^{\circ} \mathrm{C}$, deutlich niedriger als DME. Auch wenn Toftmann et al. [124] für die 

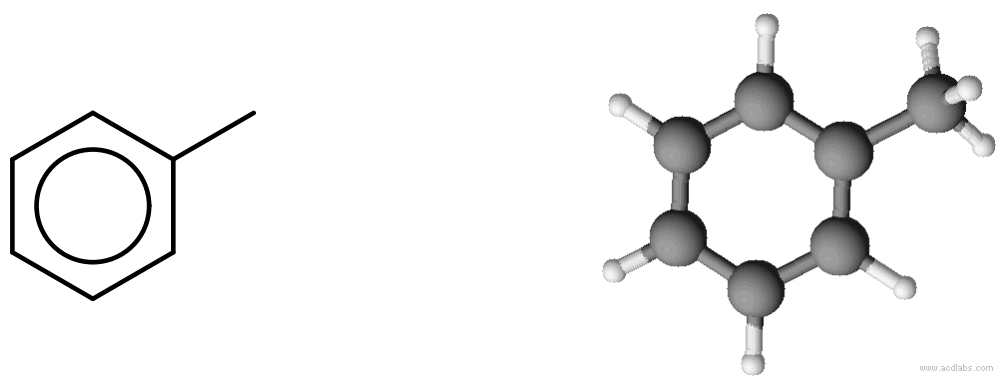

Abbildung 5.33: Strukturformel von Toluol

Laserdepositon von gefrorenen, reinen Toluol-Targets mittels $193 \mathrm{~nm}$ Lasern eine deutliche Filmbildung beobachten und für $248 \mathrm{~nm}$ Laser immerhin Anzeichen davon erkennen, scheint diese Matrix generell vielversprechend zu sein.

Filme, die mittels MAPLE mit Toluol mit 2 Gew.\% PMMA hergestellt wurden, zeigen wiederum als einzige Färbung Schichtdickeninterferenzen und hinterlassen nach dem Lösen in THF keine sichtbaren Rückstände. Vergleicht man die Infrarotspektren mit denen von PMMA (Abb. 5.34), so sieht man keine signifikanten Unterschiede. Es tritt also wiederum

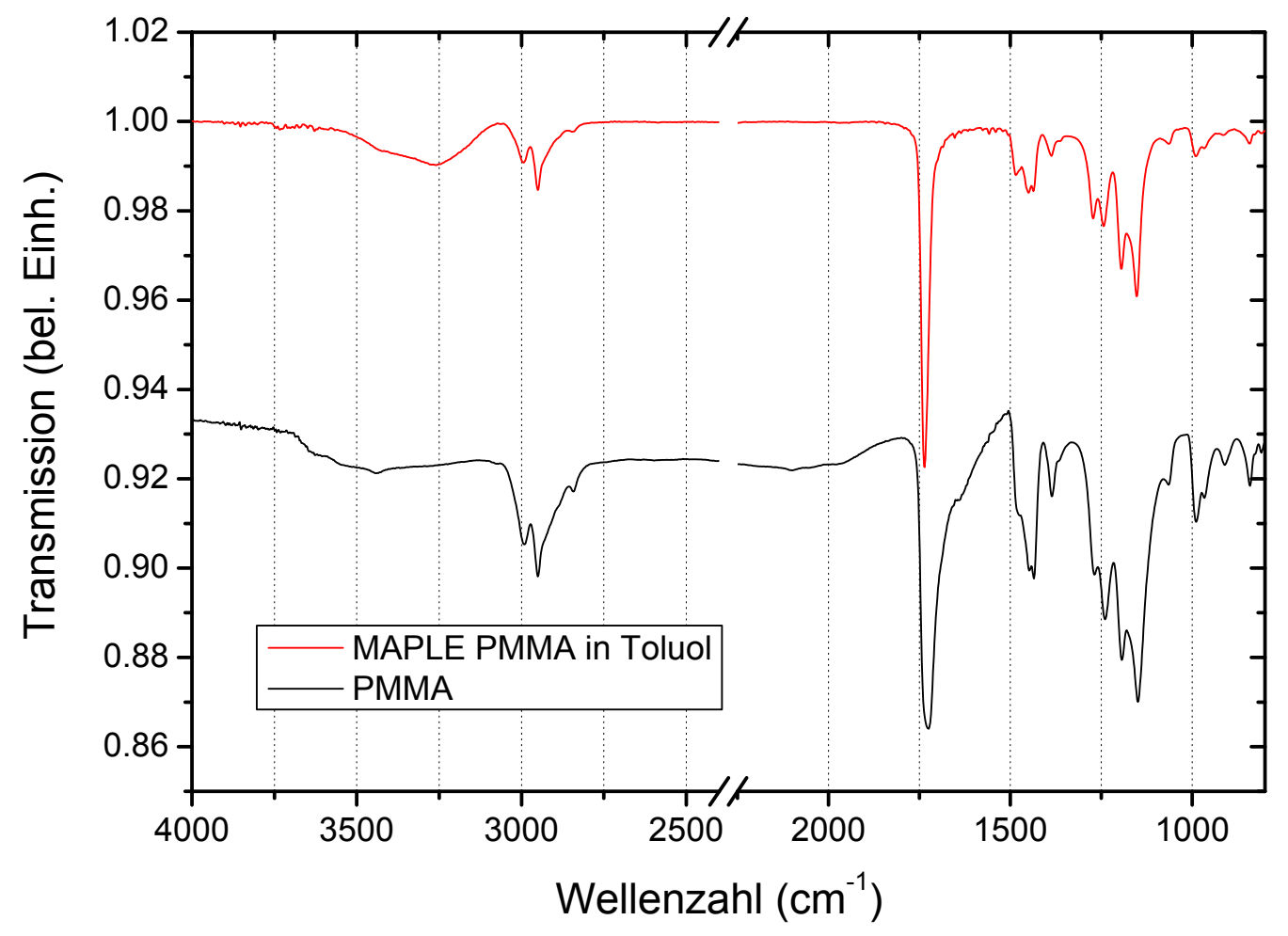

Abbildung 5.34: Infrarotspektren von MAPLE PMMA mit Toluol und von üblichem laserdeponiertem PMMA. Die Spektren wurden gegeneinander verschoben. 
keine merkliche Veränderung der chemischen Bindungsverhältnisse zum Ausgangsmaterial auf.

Betrachtet man die Proben im Lichtmikroskop (Abb. 5.35), so sieht man einen deutlichen Unterschied zu den Proben, bei denen DME als Lösungsmittel verwendet wurde. Die Proben sind übersäht mit einzelnen Droplets, die deutlich kleiner sind als bei den mit
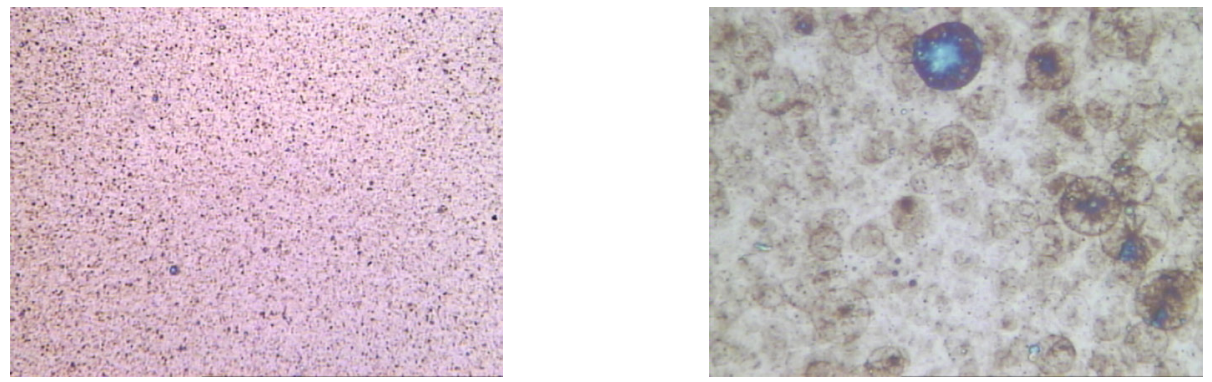

Abbildung 5.35: Lichtmikroskopaufnahmen von PMMA Filmen mittels MAPLE mit Toluol hergestellt. Links: 300 Pulse, ca. 50fache Vergrößerung; rechts: ca. 1000fache Vergrößerung

DME hergestellten und dafür dichter liegen. In höherer Vergrößerung ist zu erkennen, dass sie weitgehend rund sind.

Unter dem Rasterelektronenmikroskop ist in Abbildung 5.36 zu erkennen, dass sehr viele Droplets existieren und für diese alle Größen auftreten, die nicht, wie für PLD häufig beobachtet, bimodal verteilt sind [33]. Die auf dem rechten Bild in der Mitte sichtbare
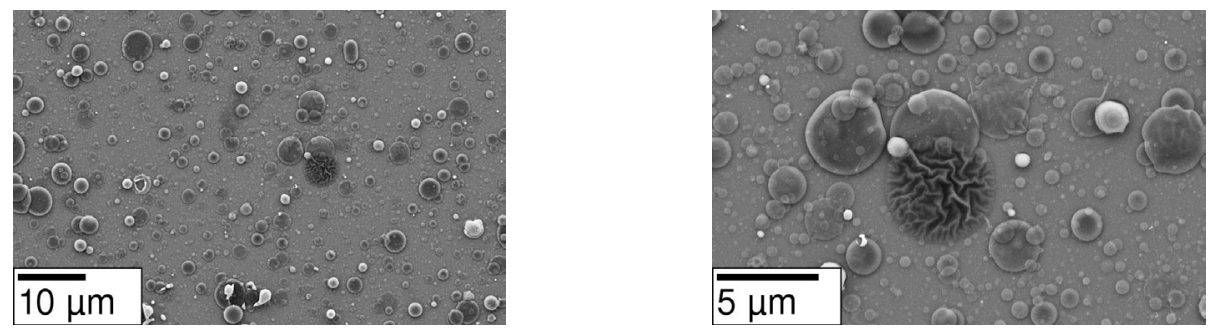

Abbildung 5.36: Rasterelektronenmikroskopaufnahmen von PMMA Filmen mittels MAPLE mit Toluol hergestellt. Links: 300 Pulse, ca. 700fache Vergrößerung; rechts: ca. 2000fache Vergrößerung

faltige Struktur ist häufiger auf den Proben zu finden wenngleich nicht repräsentativ für die gesamte Schicht. Sellinger et al. [106] beobachten diese Struktur ebenfalls und führen sie darauf zurück, dass die Tröpfchen von ihrer Oberfläche her eintrocknen und dabei eine feste Hülle bilden, die dann durch kleine Löcher Lösungsmittel ausdampfen und damit zusammenfallen. Diese Erklärung erscheint einleuchtend, auch wenn hier keine von den 
dort berichteten kleinen Löchern im SEM sichtbar gemacht werden können, selbst bei 60.000facher Vergrößerung.

Des Weiteren ist zu bemerken, dass Sellinger et al. [106] die vermehrte Bildung von Strukturen auf der Oberfläche bei PMMA Konzentrationen über 1\% im Target beobachtet haben. Eine Verringerung der Konzentration könnte hier für glattere Filme sorgen.

Bei diesen Bildern stellt sich die Frage, ob, wie bei reinem PMMA, zwischen den Droplets ebenfalls eine glatte Komponente existiert. Dies wurde mittels Röntgenreflektometrie untersucht. In Abbildung 5.37 ist zu sehen, dass hier keinerlei Kiessig-Fringes auftreten,

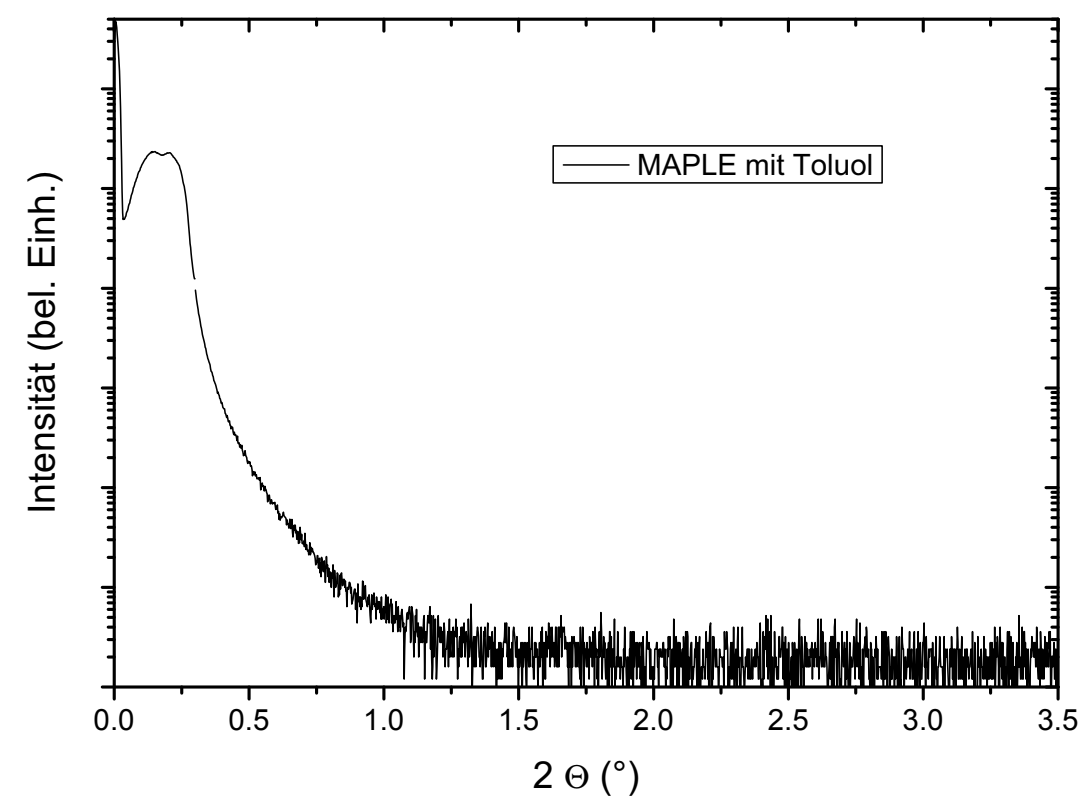

Abbildung 5.37: Röntgenreflektometriemessung eines mittels MAPLE mit Toluol deponierten PMMA Films auf Si

die auf eine glatte Schicht zurückschließen ließen. Es ist damit davon auszugehen, dass die Droplets das einzige Depositionsprodukt auf den Filmen sind. Da keine Rückstände nach Lösen in THF auftreten, ist der vernetzte Anteil auf jeden Fall sehr gering.

Die entscheidende Änderung zu normaler Laserdeposition von PMMA sieht man in der Molmassenverteilung des Films. Diese ist in Abbildung 5.38 aufgetragen. Wenn man der chromatographischen Analyse auch bei sehr kleinen Molmassen quantitativ glaubt, liegt der Anteil an Molekülen mit Molmasse über $2000 \mathrm{~g} / \mathrm{mol}$ bei über 98,5\%. Leider ist diese Angabe nicht sehr sicher, da zum einen die Analyse für Molmassen deutlich unter $1000 \mathrm{~g} / \mathrm{mol}$ sehr ungenau ist, zum anderen ist nur schwer eine korrekte Basislinie anzugeben, da viele miteinander überlagerte Peaks existieren. Die mittlere Molmasse des langkettigen Anteils 
liegt bei $M_{\mathrm{W}}=28 \mathrm{~kg} / \mathrm{mol}$. Dies lässt auf eine radikale Veränderung des Ablationsprozesses schließen.

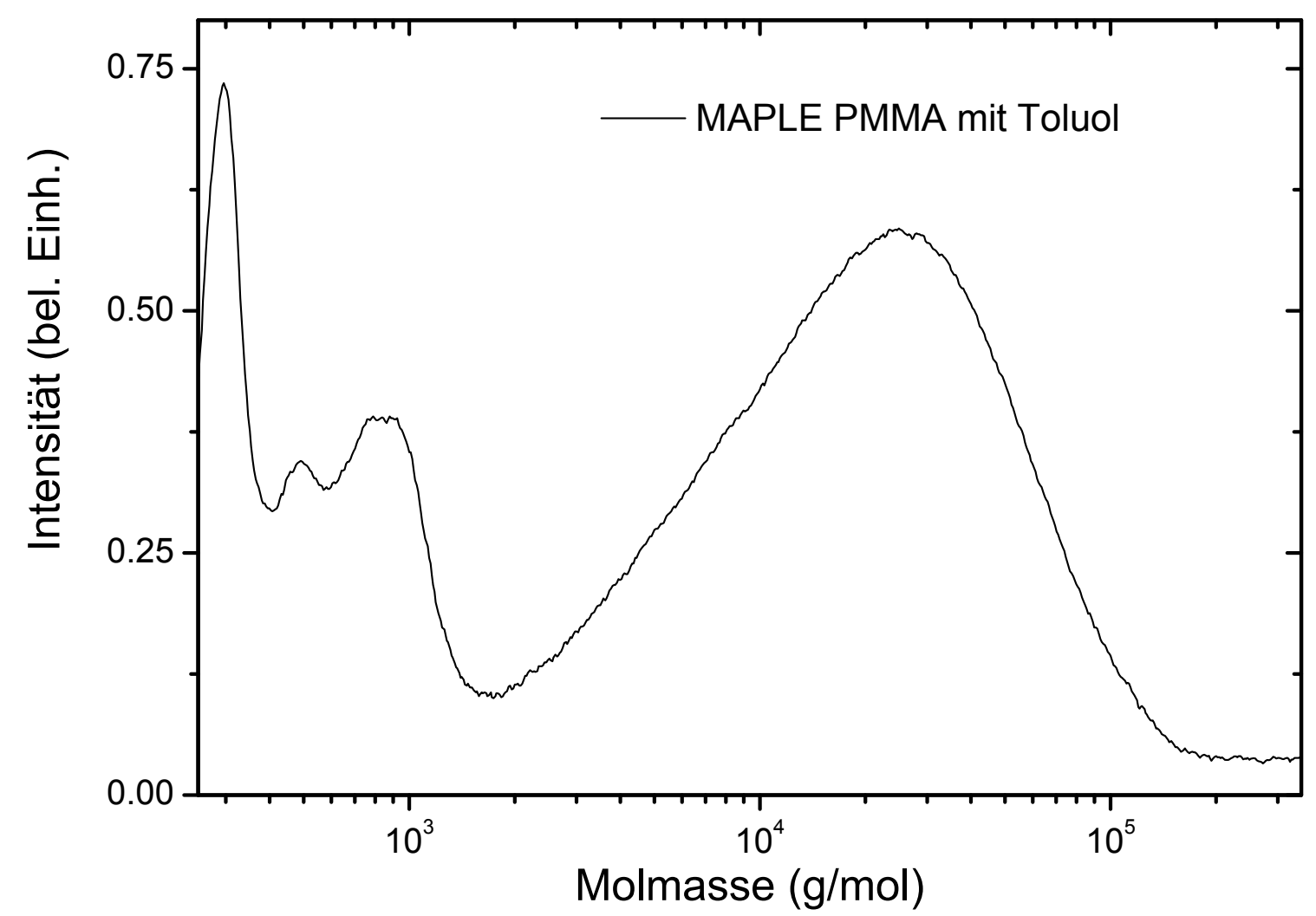

Abbildung 5.38: SE-Chromatographie von Filmen aus MAPLE PMMA mit 2\%iger Lösung in Toluol

Damit ist zu vermuten, dass bei einer noch stärkeren Veränderung des Ablationsprozesses in diese Richtung höhere Kettenlängen zu erreichen sind. Aus diesem Grund wurde das gleiche Verfahren mit einer 0,5\%igen PMMA Lösung in PMMA verwendet. Die hier entstehende SEC Kurve ist in Abbildung 5.39 zu sehen. Die mittlere Molmasse der langkettigen Komponente steigt noch einmal auf $M_{W}=48 \mathrm{~kg} / \mathrm{mol}\left(M_{N}=28,5 \mathrm{~kg} / \mathrm{mol}\right)$. Allerdings steigt der Anteil der kurzkettigen Moleküle wieder auf über 10\%, was wieder auf die ungenaue Analyse zurückzuführen sein könnte. Eine weitere Senkung des Polymeranteils hat dagegen keinen Effekt auf die Kettenlänge, bei der Deposition aus einer 0,1\%-igen PMMA-Lösung bleibt $M_{W}$ bei $48 \mathrm{~kg} / \mathrm{mol}$.

Offensichtlich unterscheidet sich der Mechanismus der Ablation und Deposition mit dieser Methode deutlich von dem der Laserdeposition unter Standardbedingungen und auch 


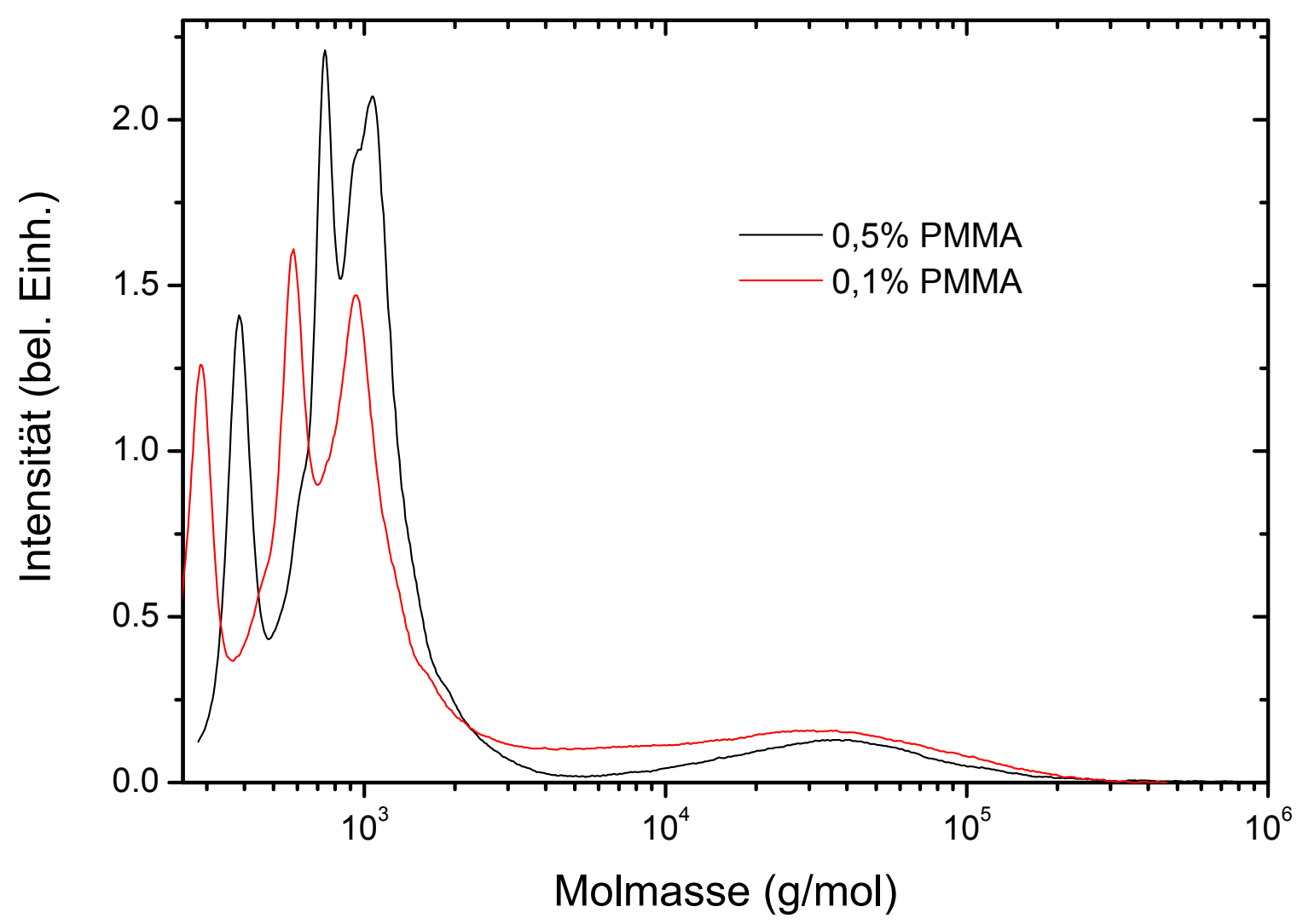

Abbildung 5.39: SE-Chromatogramme von Filmen aus MAPLE PMMA mit 0,5\%iger und 0,1\%iger Lösung in Toluol

von dem von MAPLE mit dem Lösungsmittel DME. Der langkettige Anteil ist deutlich stärker, was bedeutet, dass der Schutz des Probenmaterials durch das Lösungsmittel gut funktioniert. Die beobachteten Produkte sind jedoch weiterhin Droplets, die aus flüssigen Lösungströpfchen entstanden sind. Die Interpretation des Mechanismus mit der festen Haut auf der Oberfläche der Flüssigkeitströpfchen bedeutet aber, dass das Verdampfen der Flüssigkeit schneller geschieht, als die Konvektion innerhalb des Tröpfchens die Homogenität wieder herstellen könnte und so zur Nukleation eines einzelnen, eher runden Partikels führen könnte. Letzteres ist vermutlich bei der MAPLE Deposition mit DME der Fall, wo kleine, runde Kugeln in der Mitte der Trockenflecken beobachtet wurden. Der Unterschied zwischen den beiden Lösungsmitteln liegt hier in dem deutlich unterschiedlichen Dampfdruck.

Damit bleibt festzustellen, dass durch die Verwendung der MAPLE Methode mit dem hier neu als Matrix eingeführten Toluol PMMA deponierbar ist, das wesentlich langkettiger 
ist als für die übliche gepulste Laserdeposition. Auch die physikalischen Eigenschaften wie Löslichkeit und Transparenz sind wesentlich näher am Ausgangsmaterial. Vor allem die Kettenlänge der Makromoleküle lässt sich bedeutend erhöhen.

Negativ anzumerken ist, dass die sonst von der PLD bekannte hervorragende Substrathaftung verringert ist, hier könnte man sicherlich mit einer Kombination, die erst mit normaler PLD arbeitet und dann auf ein gefrorenes Target wechselt, Abhilfe schaffen.

Insgesamt ist der Parameterraum für MAPLE zu unüberschaubar, um aus dieser kurzen Studie sagen zu können, wie groß das Potential optimal ist. Untersucht wurden hier Auswirkungen des verwendeten Lösungsmittels, die Konzentration und ansatzweise der Gasdruck, der Target-Substrat-Abstand und die Laserenergiedichte. Punkte wie Target-Temperatur [16], Substrattemperatur [93] und Laserwellenlänge [124] spielen zusätzlich eine Rolle, wurden aber hier nicht betrachtet. Es ist zu vermuten, dass durch eine Optimierung aller Parameter die chemische Zusammensetzung des Polymers weitgehend unbeschadet übertragen werden kann. Dies war allerdings nicht das Ziel dieser Untersuchung. Hier konnte gezeigt werden, dass durch eine radikale Veränderung der Absorption im Target die Zerstörung des Polymers deutlich reduziert werden kann. Allerdings sind dazu sehr hohe Konzentrationen an Absorbern notwendig, so dass tatsächlich nur ein sehr geringer Anteil an ursprünglichem Targetmaterial übrig bleibt.

Der nächste Schritt für eine Anwendung des MAPLE Verfahrens wird sein, das stickstoffgekühlte Target während der Herstellung zu bewegen, um eine Abrasterung zu erreichen. Auf diese Weise sollte es möglich sein, weit einfacher Filme besserer Qualität herzustellen. Auch eine Optimierung der Laserenergie, z. B. durch Verwenden einer abbildenden Lasergeometrie mit entsprechenden Strahlabschwächern wäre wünschenswert, um diesen Parameter genauer untersuchen zu können. Ein weiterer Punkt, der hier nur am Rande untersucht werden konnte, ist die Qualität des Targets. Von der PLD ist bekannt, dass Targets homogen und dicht sein müssen, eine Eigenschaft, die bei den schockgefrorenen Targets zu verbessern ist. 


\section{Mechanische Eigenschaften von laserdeponiertem PMMA}

Die quantitative Bestimmung der mechanischen Eigenschaften eines Materials, speziell des komplexen Elastizitätsmoduls, ist nicht trivial, da dieser sowohl von der Temperatur als auch von der betrachteten Messzeit abhängt. Für kristalline Festkörper ist im Allgemeinen eine recht klare Trennung zwischen plastischem und elastischem Anteil festzustellen. Bei amorphen Stoffen ist dieses Verhalten deutlich komplexer, die Zeitabhängigkeit ist deutlich stärker ausgeprägt, und die Mechanismen sind auch heute von zentralem Interesse in der Forschung. In diesem Kapitel sollen zunächst die Grundlagen des mechanischen Verhaltens von Polymeren kurz aufgeführt werden. Darauf sollen die mittels des Plasma Puls angeregten Reed (PPXR) (siehe Kapitel 3) am System PMMA gefundenen Resultate gezeigt und die Ergebnisse diskutiert werden.

\subsection{Grundlagen}

Die Beschreibung der temperaturabhängigen Eigenschaften amorpher Feststoffe beschäftigt die Wissenschaft schon seit vielen Jahrzehnten und bis heute ist kein geschlossenes Bild erkennbar. Im folgenden sollen jetzt einige der für diese Arbeit wichtigsten Ergebnisse kurz zusammengefasst werden.

\subsubsection{Qualitative Befunde}

Zentrales Thema der Materialphysik ist der Zusammenhang makroskopischer Eigenschaften mit den mikroskopischen Vorgängen bis zur atomaren Skala. Auch für Polymere sind für die makroskopischen Eigenschaften mikroskopische Prozesse maßgeblich. Anders als für Kristalle kann hier, gerade für amorphe Polymere, Bewegung nicht über Versetzungsgleiten stattfinden. Ein Äquivalent hierzu führt de Gennes mit dem Reptationsmodell ein [25]. Die Bewegung erfolgt hier über die Bewegung ganzer Ketten. Für diese bilden die umgebenen Moleküle enge Kanäle, durch die sich die betrachtete Kette hindurchschlängelt (Abb. 6.1 links). Sie kann folglich im Wesentlichen nur in einer Dimension bewegt werden. Durch das 


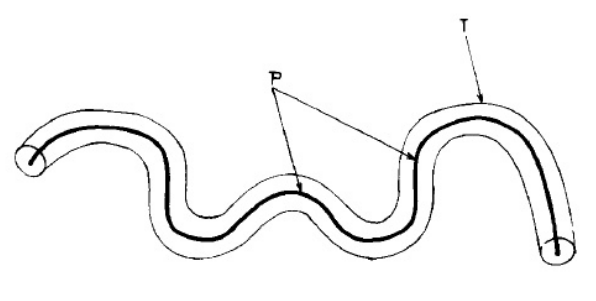

(a)

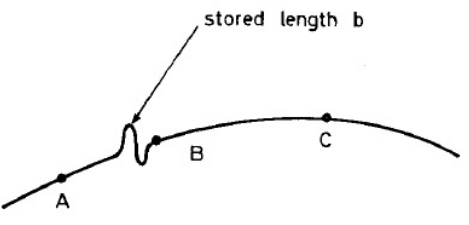

(b)

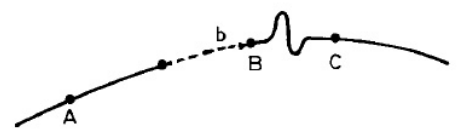

Abbildung 6.1: Reptationsmodell nach de Gennes [25]. Links: Die Kette (P) ist in einer Röhre (T) gefangen und kann sich nur in einer Dimension bewegen; Rechts: Durch Schleifen kann potentielle Länge gespeichert und bewegt werden.

Bilden von Schleifen ohne eingeschlossene andere Ketten kann mikroskopisch Länge für die makroskopische Dehnung gespeichert werden (Abb. 6.1 rechts). Der Betrag dieser im Gleichgewicht gespeicherten Länge ergibt sich durch die Entropie, ist damit temperaturabhängig und die Wiederherstellung benötigt eine charakteristische Relaxationszeit. Das ständige dynamische Umformen der Kanäle und die niedrige intermolekulare Bindungsenergie der Polymerketten unterstützt die Zeitabhängigkeit der Bewegungsprozesse und führt dazu, dass echt-elastisches Verhalten in unvernetzten Polymeren über lange Beobachtungszeiträume kaum beobachtet wird. Es tritt im wesentlichen viskoses und viskoelastisches Verhalten auf.

Alle diese Bewegungen können, wie für die Festkörpertheorie üblich, statistisch beschrieben werden, indem ein schwingendes Teilchen auf eine Potentialbarriere trifft und diese nur mit einer gewissen Wahrscheinlichkeit überschreitet. Allerdings sind die hier zu betrachtenden Anklopffrequenzen teilweise sehr viel kleiner, da es sich nicht um einzelne Atome handelt, die mit einer Debye-Frequenz in der Größenordnung von $10^{14} \mathrm{~Hz}$ schwingen, sondern um Makromoleküle mit Massen von bis zu einigen $10^{6} \mathrm{~g} / \mathrm{mol}$, von denen Segmente schwingen $\left(\tau_{\alpha}=1,48 \cdot 10^{-11}\right.$ s für syndiotaktisches PMMA mit $M_{w}=50.000$ [6]).

Ein sehr erfolgreiches Modell für die Beschreibung von Gläsern ist die Beschreibung über multidimensionale Energielandschaften [114], die mit der Modenkopplungstheorie [5, 71] verbunden ist. Dabei wird das Glas als N-dimensionale variable Potentiallandschaft beschrieben, in der sich die Teilchen bewegen. Bei geringerer Temperatur sinkt die mittlere Teilchenenergie und die Wahrscheinlichkeit, dass Potentialbarrieren überwunden werden. Eine sehr interessante Erklärung für den Glasübergang ist nun, dass unterhalb der Glastemperatur die Wahrscheinlichkeit, dass größere Umwandlungen in der Potentiallandschaft 
auftreten, gegen Null geht, und damit nur noch kleinere Sprünge, verbunden mit kleineren Umwandlungen auftreten. Im Besonderen werden kooperative Umordnungen größerer Bereiche unterdrückt. An diesem Punkt ändert sich das System auf einen nicht-ergodischen Zustand [23].

Dieses Modell ist auch auf Gläser komplexerer Moleküle wie Polymere anwendbar. Allerdings gibt es in komplexen Makromolekülen, anders als bei Atomen, eine Vielzahl weiterer Freiheitsgrade. Auf diese Weise können zusätzliche Dreh-, Schwingungs- und Verschiebungsbewegungen stattfinden, von denen einige einen nicht unerheblichen Teil an Energie aufnehmen können, auch wenn sie zur makroskopischen Bewegung nicht beitragen.

Die auftretenden Relaxationsmechanismen werden in der Reihenfolge ihrer charakteristischen Frequenzen als $\alpha$-, $\beta$ - und ggf. $\gamma$ - Relaxationen nummeriert. Für das hier verwendete PMMA beobachtet man die $\alpha$-Relaxation, die mit dem Glasübergang und der Bewegung ganzer Ketten, hauptsächlich Kippschwingungen und Rotationen von Kettensegmenten, die die Gesamtbewegung unterstützen, verbunden ist 64]. Zusätzlich tritt die $\beta$-Relaxation auf, die mit der Bewegung der gesamten Ester-Seitengruppe, hauptsächlich einer Rotationsbewegung in zwei Hauptpositionen um $180^{\circ}$ mit zusätzlichen Ausgleichs- und Kippschwingungsbewegungen auch den Bindungen der Hauptkette, verbunden ist [104]. Weiterhin wird eine durch Tunneln bestimmte Rotation der Methylgruppe beobachtet, jedoch bei Temperaturen im Bereich von $-120{ }^{\circ} \mathrm{C}$ (bei $360 \mathrm{~Hz}$ ) [134], was außerhalb des hier betrachteten Beobachtungsbereichs liegt. Diese müsste konsequenterweise als $\gamma$-Relaxation gezählt werden, wird jedoch wegen ihrer geringen Bedeutung in der Literatur meist ignoriert.
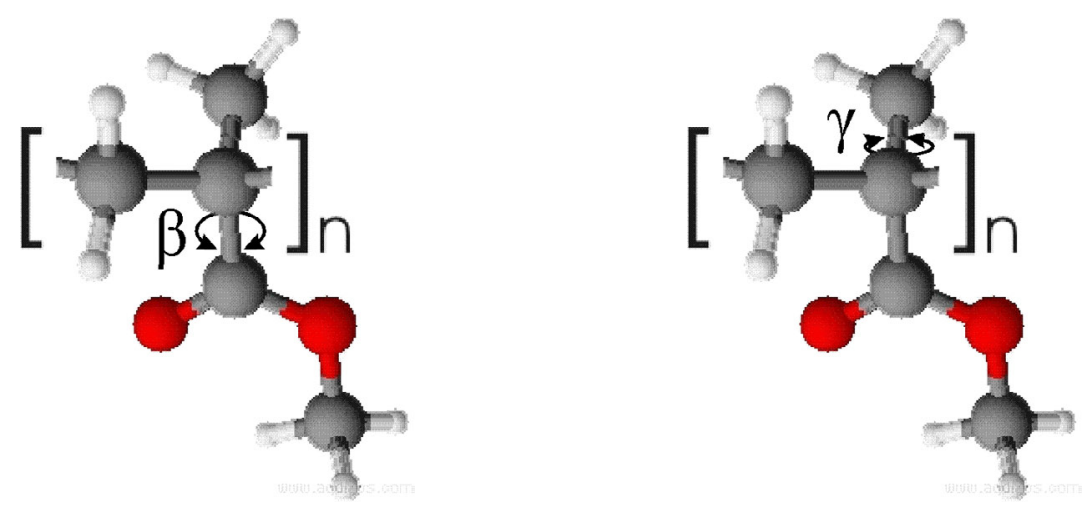

Abbildung 6.2: Hauptschwingungen für die $\beta$ - und $\gamma$-Relaxation

Da die $\alpha$ - und $\beta$ - Relaxationsvorgänge ein unterschiedliches Temperaturverhalten der charakteristischen Zeiten zeigen, liegen sie bei Temperaturen von etwa $120{ }^{\circ} \mathrm{C}$ im gleichen 
Frequenzbereich von einigen tausend $\mathrm{Hz}$ und die beiden deutlich mehr als eine Dekade breiten Frequenzkurven überschneiden sich (merging) [42, 6]. Betrachtet man umgekehrt die Relaxationsvorgänge, wie in dieser Arbeit geschehen, bei einer festen Frequenz, treten sie bei derselben Temperatur auf. Dieses Phänomen erschwert die Beobachtung, da die Trennung der beiden Mechanismen sehr schwierig ist. Eine detailliertere Diskussion für die hier durchgeführten Experimente findet in Abschnitt 6.4 statt.

\subsubsection{Beobachtungen in der Zeitdomäne}

Wie schon oben beschrieben, ergeben sich für die unterschiedlichen Relaxationsmechanismen unterschiedliche charakteristische Relaxationszeiten $\tau$. Die einfachste Möglichkeit der Beschreibung des Relaxationsverhaltens ist eine Potentialbarriere fester Höhe wie im Debye-Modell des Festkörpers mit einem mit fester Frequenz anklopfenden Teilchen. Damit ergibt sich auf eine stufenförmige Anregungsfunktion die makroskopische Antwort

$$
\varphi_{\mathrm{DB}}(t)=\varphi_{0} \exp \left(-\frac{t}{\tau_{\mathrm{DB}}}\right)
$$

mit der Zeit $t$ und der Relaxationszeit $\tau_{\mathrm{DB}}$ [24]. Wie schon Debye 1913 in der Betrachtung der Dispersionsrelationen von Wasser feststellt, führt dieses zu einem breiten Maximum des Verlustmoduls im Frequenzraum für $\omega \tau \approx 1$. Gleichzeitig ergibt sich ein Abfall im Realteil der komplexen Antwortfunktion (in unserem Fall dem Elastizitätsmodul).

Dieses einfache Gesetz für die Antwortfunktion gilt allerdings nur, wenn es nur eine einzige Relaxationszeit gibt. Eine gute Annäherung erhält man, wenn die unterschiedlichen zur Relaxation führenden Ereignisse voneinander unabhängig und außerdem sehr ähnlich sind.

Für die $\alpha$-Relaxation geht man davon aus, dass die Einzelprozesse kooperative Umordnungen von ganzen Bereichen erfordern ([68] für metallische Gläser). Da diese sich unterscheiden, hängt die genaue Relaxationszeit von der konkreten mikroskopischen Umgebung ab, die nie identisch ist. Damit ergibt sich eine Verteilung von Relaxationszeiten, die eine Verbreiterung der Antwortfunktion ergibt. Analytisch exakte Lösungen sind hierfür nicht bekannt, da die genaue Verteilung der Relaxationszeiten unbekannt ist. Stattdessen ver- 
wendet man üblicherweise die gestreckte Exponentialfunktion nach Kohlrausch, Williams und Watts (KWW) [57, 133]

$$
\varphi_{\mathrm{KWW}}=\varphi_{0} \exp \left(\left(-\frac{t}{\tau_{\mathrm{KWW}}}\right)^{\beta_{\mathrm{KWW}}}\right),
$$

bei der die Funktion aus Gleichung 6.1 um einen Exponenten $\beta_{\mathrm{KWW}}$ mit Werten zwischen 0 und 1 gestreckt wird. Gleichung 6.2 kann durch ein Integral über eine Dichte von Debyeschen Anregungsfrequenzen exakt beschrieben werden

$$
\varphi_{0} \exp \left(\left(-\frac{t}{\tau_{\mathrm{KWW}}}\right)^{\beta_{\mathrm{KWW}}}\right)=\int_{0}^{\infty} \exp \left(\frac{-t}{\tau_{\mathrm{DB}}}\right) \rho\left(\tau_{\mathrm{DB}}\right) d \tau_{\mathrm{DB}} .
$$

Die Berechnung der Verteilung der Relaxationszeiten $(\rho(\tau))$ ist jedoch nicht trivial und i. A. nur numerisch und vor allem nicht eindeutig lösbar [1].

\subsubsection{Temperaturabhängigkeit}

Zusätzlich ergibt sich die Schwierigkeit, dass auch die in den Antwortfunktionen benutzte Relaxationszeit $\tau$ temperaturabhängig ist. Im einfachsten Fall nimmt man dafür ein Arrhenius-Verhalten an:

$$
\tau_{\text {Arr }}=\tau_{0} \exp \left(\frac{E}{k_{B} T}\right) .
$$

Dieses beschreibt sehr gut einfachere Relaxationen wie die $\beta$-Relaxation.

In vielen Fällen, gerade für komplexere Vorgänge wie die $\alpha$-Relaxation, passt die VogelFulcher-Tammann Gleichung (VFT) [132, 41, 122] besser:

$$
\tau_{\mathrm{VFT}}=\tau_{0} \exp \left(\frac{D T_{\mathrm{VFT}}}{T-T_{\mathrm{VFT}}}\right) .
$$

Der Parameter $D$ beschreibt die Verbreiterung der Funktion. Wegen der Divergenz bei $T=T_{\mathrm{VFT}}$ funktioniert diese Beschreibung nur für $T>T_{\mathrm{VTF}}$. Auch für diese Verteilung werden kooperative Prozesse sowie die Verteilung der Bindungsverhältnisse in dem Material verantwortlich gemacht. Angell teilt Gläser nach ihrer Abweichung zum ArrheniusVerhalten in starke (Arrhenius-artige, z. B. $\mathrm{SiO}_{2}$ ) und fragile (z. B. PMMA) Gläser ein [4]. Es gibt einen einfachen Zusammenhang zwischen dem Breiteparameter $D$ und dem Fragilitätsindex nach Angell [30]. 


\subsection{4 Übergang zur periodischen Anregung}

Der Übergang zwischen dem Zeit- und dem Frequenzraum wird durch eine Fourier-Transformation erreicht. Leider lassen sich komplexere Funktionen meist nicht geschlossen transformieren, so dass i. A. weitere empirische Funktionen eingeführt werden. Nur für die DebyeAntwortfunktion (Gleichung 6.1] lässt sich eine exakte Lösung der komplexen Relaxationsfunktion $\chi^{*}$ im Frequenzraum finden:

$$
\chi^{*}(\omega)_{\mathrm{DB}}=\chi_{\infty}+\frac{\chi_{S}-\chi_{\infty}}{1+i \omega \tau_{\mathrm{DB}}}
$$

Dabei sind die Werte $\chi_{S}$ und $\chi_{\infty}$ die asymptotischen Werte für den statischen Fall bzw. denjenigen für unendliche Frequenz.

Für Fälle komplizierterer Relaxation, in denen eine Debye-Dynamik das tatsächliche Verhalten nicht ausreichend beschreibt, wird die Havriliak-Negami (HN) Funktion eingeführt [49]

$$
\chi^{*}(\omega)_{\mathrm{HN}}=\chi_{\infty}+\frac{\chi_{S}-\chi_{\infty}}{\left(1+\left(i \omega \tau_{\mathrm{HN}}\right)^{\alpha}\right)^{\gamma}} .
$$

Die beiden neu eingeführten Parameter sind die Verbreiterung $\alpha$ und die Asymmetrie $\gamma$, beide zwischen 0 und 1. Für diese wurden häufig Spezialfälle verwendet, $\alpha=1$ nennt sich die Cole-Davidson-Funktion [21, 22], $\gamma=1$ Cole-Cole-Funktion [20]. Seitdem die Anpassung über leistungsfähigere Computer erfolgt, werden jedoch meist beide Parameter mit gutem Erfolg variiert. Bei der HN-Funktion handelt es sich zwar nicht um eine exakte FourierTransformation der KWW-Funktion (Gleichung 6.2), bei geeigneter Wahl der Parameter sind sie jedoch sehr ähnlich. Dabei gilt meist $\alpha \gamma=\beta_{\mathrm{KWW}}^{1,23}$ [1, 2].

Für die praktische Anwendung ist es wichtig, die Havriliak-Negami Funktion in den Real- und Imaginärteil aufzuspalten. Diese lauten:

$$
\begin{aligned}
\chi^{\prime}(\omega) & =\chi_{\infty}+\left(\chi_{s}-\chi_{\infty}\right) \frac{\cos (\gamma \varphi)}{\left(1+2\left(\omega \tau_{\mathrm{HN}}\right)^{\alpha} \cos \left(\alpha \frac{\pi}{2}\right)+\left(\omega \tau_{\mathrm{HN}}\right)^{2 \alpha}\right)^{\frac{\gamma}{2}}} \\
\chi^{\prime \prime}(\omega) & =\left(\chi_{\infty}-\chi_{s}\right) \frac{\sin (\gamma \varphi)}{\left(1+2\left(\omega \tau_{\mathrm{HN}}\right)^{\alpha} \cos \left(\alpha \frac{\pi}{2}\right)+\left(\omega \tau_{\mathrm{HN}}\right)^{2 \alpha}\right)^{\frac{\gamma}{2}}}
\end{aligned}
$$

mit

$$
\varphi=\arctan \left(\frac{\left(\omega \tau_{\mathrm{HN}}\right)^{\alpha} \sin \left(\alpha \frac{\pi}{2}\right)}{1+\left(\omega \tau_{\mathrm{HN}}\right)^{\alpha} \cos \left(\alpha \frac{\pi}{2}\right)}\right) .
$$

Für eine ausführliche Herleitung siehe [97]. 


\subsection{Temperaturabhängiger komplexer Elastizitätsmo- dul des laserdeponierten PMMA}

Zur Bestimmung des komplexen Elastizitätsmoduls verschiedener PMMA-Filme wurden auf ein Reed-Substrat mit etwa $7 \mathrm{~mm}$ Länge, von dem zuvor mittels einer Leermessung Frequenz und Dämpfung bestimmt wurden, nacheinander unterschiedlich dicke PMMAFilme bei $150{ }^{\circ} \mathrm{C}$ Substrattemperatur deponiert und nach jeder Deposition die mechanischen Eigenschaften gegen die Temperatur gemessen. Die durch die Auswertung der Schwindungsdauer (Abb. 6.3) gemessenen Schichtdicken (siehe Abschnitt 3.7) liegen bei

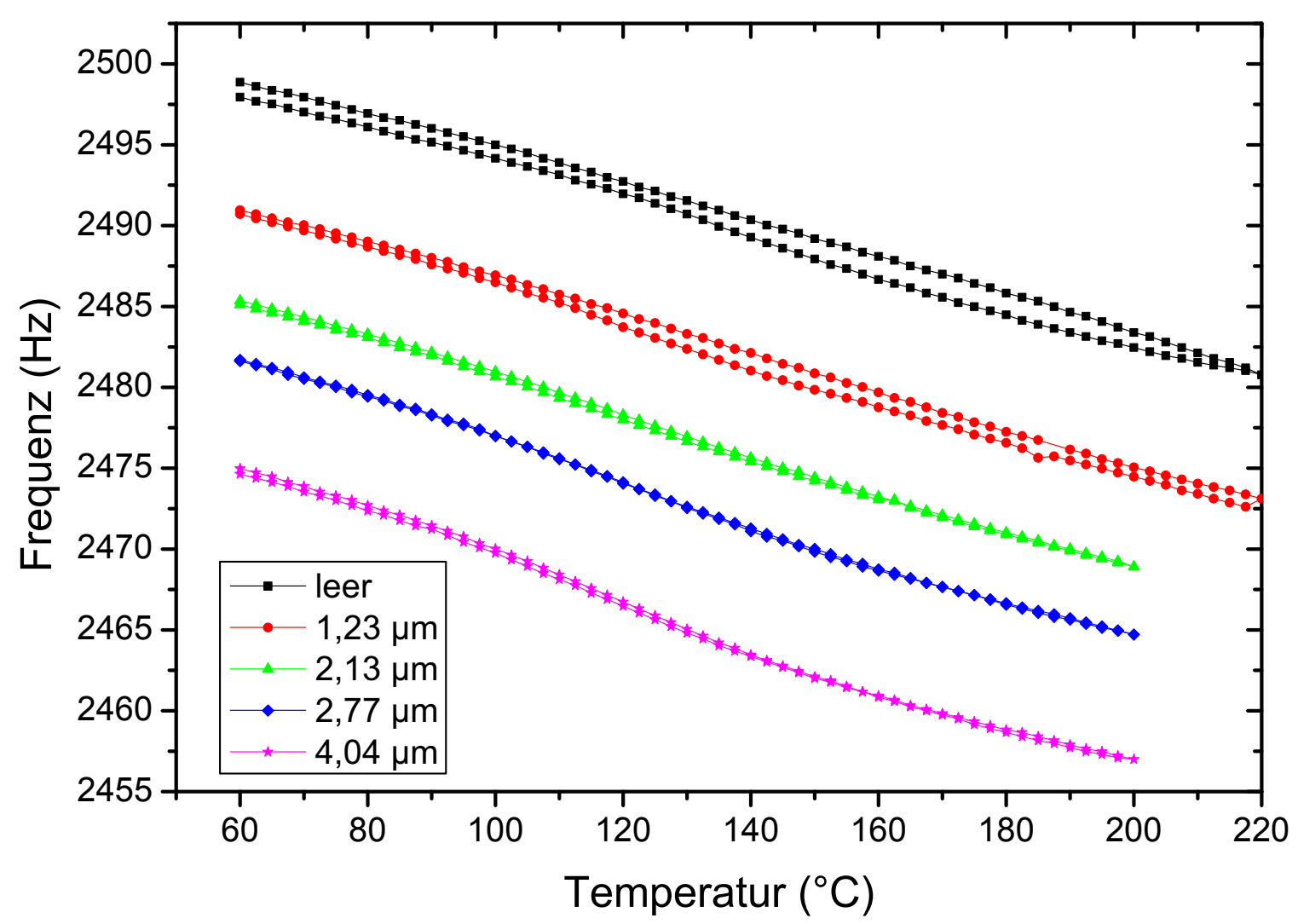

Abbildung 6.3: Mit PPXR gemessene Frequenzen gegen die Temperatur für das leere Substrat und vier verschiedene Schichtdicken

1,23, 2,13, 2,77 und 4,04 $\mu \mathrm{m}$. Vor jeder Messung wurde der bis dahin deponierte Film für 10 Minuten bei $210{ }^{\circ} \mathrm{C}$ ausgelagert. Damit wurde zum einen vermieden, den Effekt der Relaxation als Überlagerung der eigentlichen Messung zu erhalten, zum anderen wurden alle Lagen des schrittweise aufgebauten Films in einen möglichst vergleichbaren Relaxati- 
onszustand gebracht. Die erste Schicht $(1,23 \mu \mathrm{m})$ wurde wie das leere Substrat bis $220{ }^{\circ} \mathrm{C}$ gemessen, die darauf folgenden Schichten jeweils bis $200{ }^{\circ} \mathrm{C}$. Die gemessene Güte ist in Abbildung 6.4 aufgetragen. Es ist zu sehen, dass die Messung der ersten Schicht eine deutliche

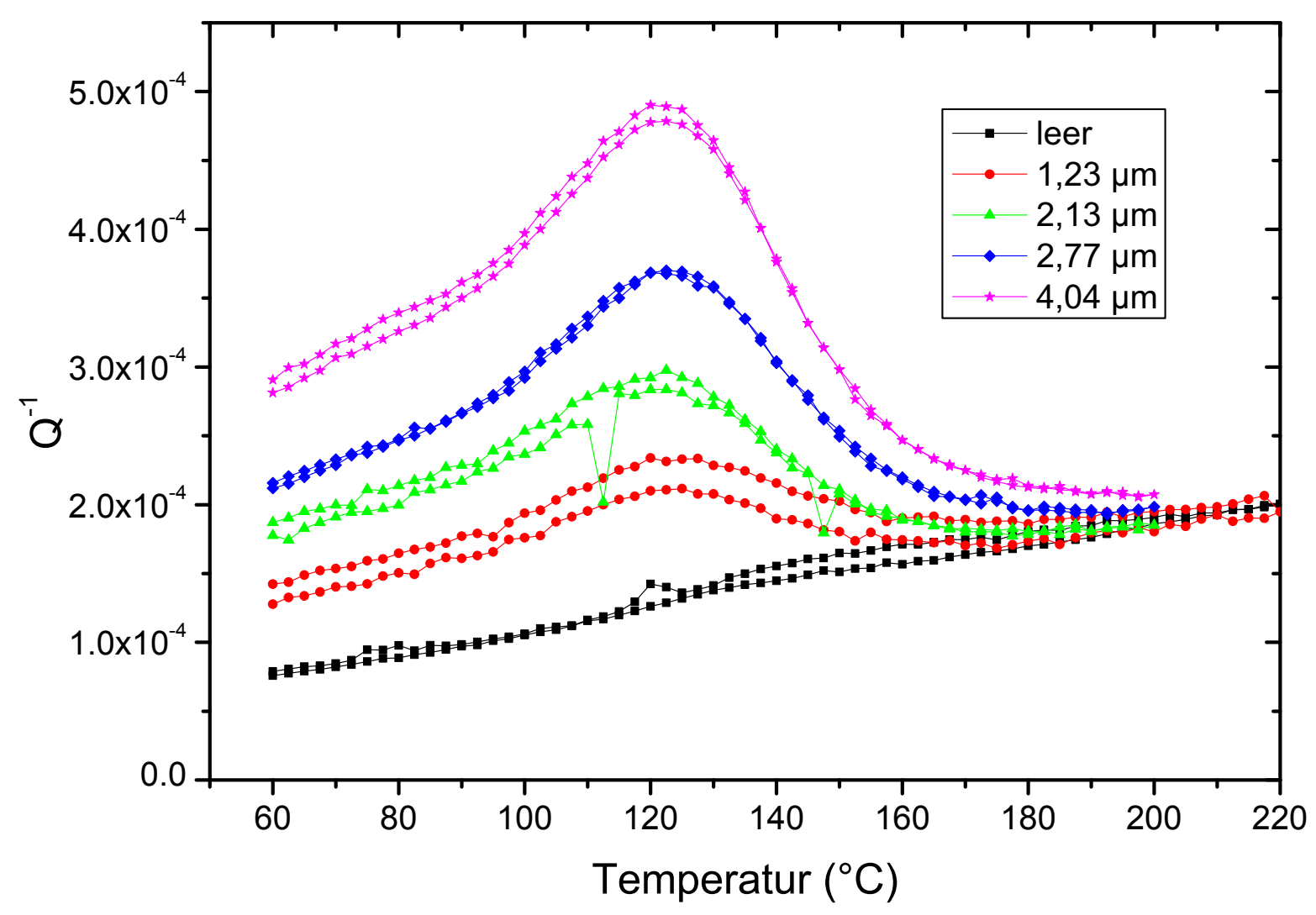

Abbildung 6.4: Mit PPXR gemessene Güten gegen die Temperatur für das leere Substrat und vier verschiedene Schichtdicken

Hysterese zeigt. Diese ist auf die unzureichende Auslagerung und damit nicht komplette Relaxation zurückzuführen. Damit ist diese Schicht zur Auswertung nur schlecht geeignet. Die weiteren Messungen liegen beim Heizen und Abkühlen fast perfekt aufeinander, so dass hier davon ausgegangen werden kann, dass sowohl die Temperaturmessung sehr gut ist als auch die Reproduzierbarkeit der Messung. Ist die Temperatur der Probe während er ganzen Messung niedriger als bei der vorhergegangenen Auslagerung, tritt keine messbare Relaxation mehr ein.

Aus diesen gemessenen Daten wird nun gemäß dem in Abschnitt 3.7 beschriebenen Verfahren der Real- und Imaginärteil des komplexen Elastizitätsmoduls bestimmt. Der Realteil ist in Abbildung 6.5 aufgetragen. Es ist zu sehen, dass die Messung erwartungs- 


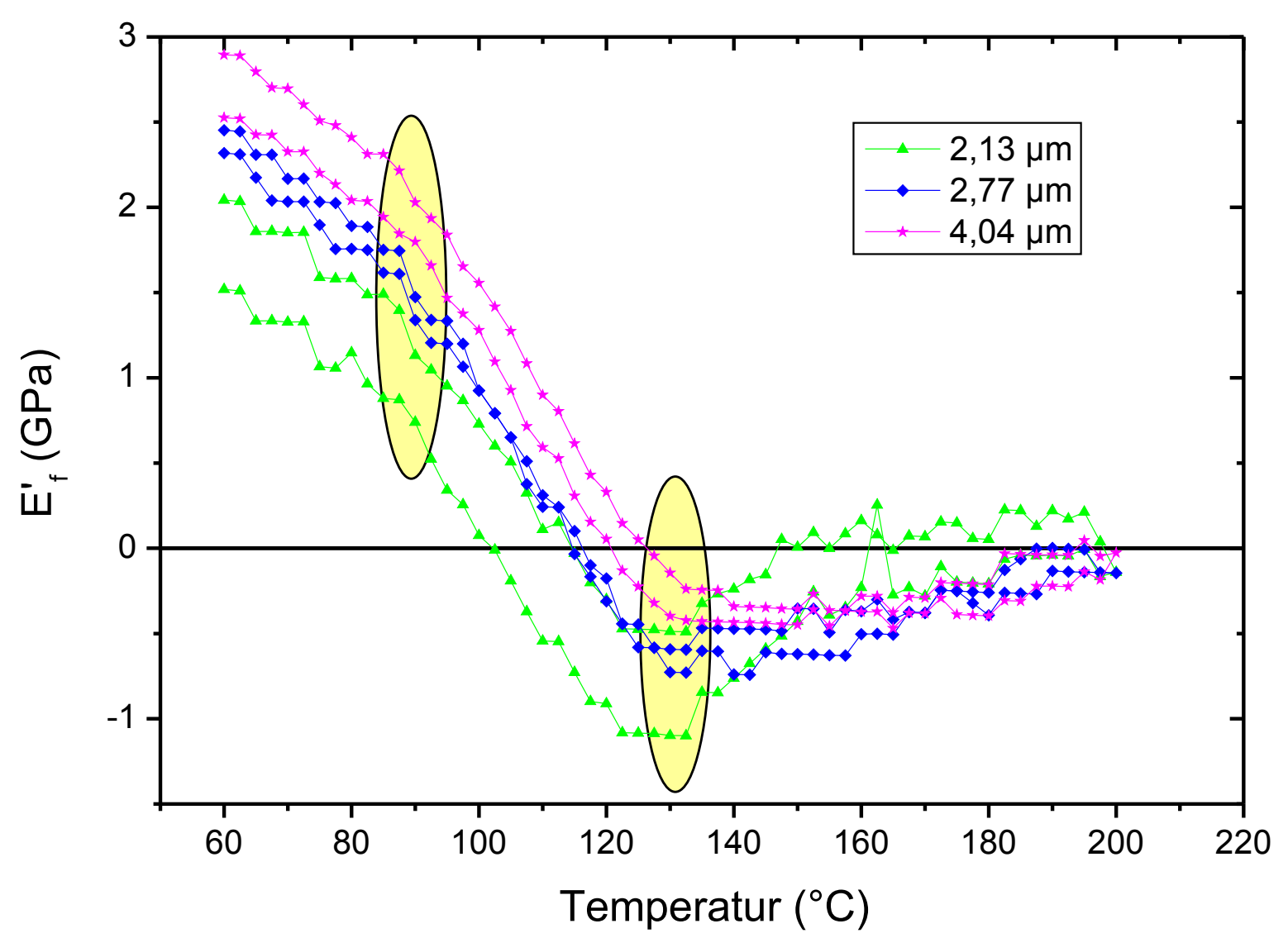

Abbildung 6.5: Berechneter Speichermodul ( $\left.E^{\prime}\right)$ für verschiedene Schichtdicken

gemäß sehr verrauscht ist, da die Messung, wie in Kapitel 3 beschrieben, hauptsächlich auf die Messung der Dämpfung und damit die Bestimmung des Verlustmoduls optimiert ist. Weiterhin wird die Resonanzfrequenz durch Querverbiegung des Substrats aufgrund von Spannungen und Torsionsmoden verändert [47]. Für die höchste Temperatur liegt der Speichermodul bis auf Rundungsfehler auf Null, was an der Anpassung der Schichtdicke liegt, wo diese Annahme eingegangen ist. Für tiefere Temperaturen ist zu sehen, dass der Speichermodul teilweise negative Werte annimmt. Dies ist unphysikalisch und nur durch die prinzipbedingte hohe Ungenauigkeit dieser Messung zu erklären. Weiterhin ist zu sehen, dass bei etwa $90{ }^{\circ} \mathrm{C}$ und bei etwa $130{ }^{\circ} \mathrm{C}$ zwei Knickpunkte auftreten. Vor dem ersten Knickpunkt, der mit der Glastemperatur zu identifizieren ist, hat die Kurve eine geringere Steigung. Da die Messung erst bei $60{ }^{\circ} \mathrm{C}$ beginnt, ist es nicht zu klären, ob diese nicht bei niedrigeren Temperaturen weiter abnimmt. Beim zweiten Knickpunkt ist der gesamte Re- 
laxationsvorgang überschritten und die Elastizität bleibt im Rahmen der Messgenauigkeit konstant bei Null.

Deutlich aussagekräftiger ist die Berechnung des Verlustmoduls in Abbildung 6.6, da die Messung hierfür optimiert ist. Es ist zu sehen, dass der Verlustmodul des Films zuerst

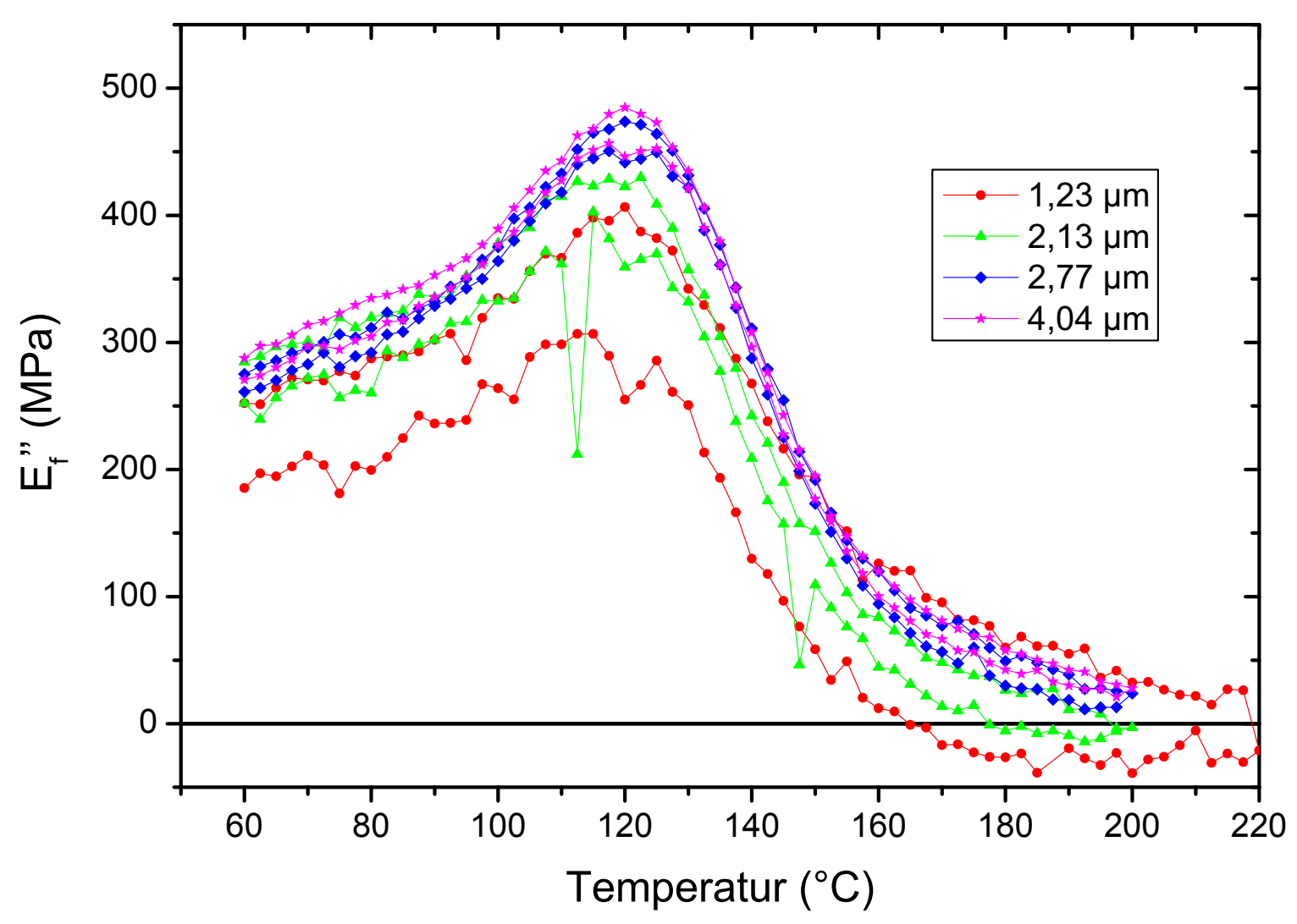

Abbildung 6.6: Berechneter Verlustmodul ( $\left.E^{\prime \prime}\right)$ für verschiedene Schichtdicken

linear ansteigt, dann bei $93{ }^{\circ} \mathrm{C}$ zu einer höheren Steigung abknickt. Es ergibt sich ein Maximum bei $122{ }^{\circ} \mathrm{C}$ und danach ein Abfall gegen Null. Auch hier ist der erste Knickpunkt mit der Glastemperatur verbunden. Nach den Kramers-Kronig-Relationen [63, 59], die $E^{\prime}$ und $E^{\prime \prime}$ verknüpfen, liegt das Maximum an der Stelle der höchsten Steigung des Speichermoduls und der Wendepunkt danach an der Stelle des dortigen zweiten Knickpunkts. Sieht man von der unsicheren Messung der dünnsten Schicht ab, erhält man für die dickeren Schichten höchstens eine kleine Veränderung des Verlustmoduls mit der Schichtdicke. Dies ist für Schichten im $\mu \mathrm{m}$ Bereich, die praktisch Volumeneigenschaften haben sollten, nicht 
anders zu erwarten. Der Verlustmodul liegt im Bereich von einigen hundert MPa, was den erwarteten Größen entspricht.

Die Anpassung einer theoretischen Form wie in Abschnitt 6.1 beschrieben, ist nicht einfach, da diese für die Messung im Frequenzraum ausgelegt sind. Hier wurde an die Kurve eine Havriliak-Negami-Funktion (Gleichung 6.8) angepasst, wobei die notwendigen Relaxationszeiten $\tau_{\text {HN }}$ durch eine Vogel-Fulcher-Tammann Funktion 6.4 genähert wurden (siehe auch [97]). Auf diese Weise werden die Daten zuerst in den Frequenz-Raum übersetzt und dann die HN-Funktion angepasst. Es wurde nur eine HN-Funktion für einen Relaxationsvorgang, in diesem Fall die $\alpha$-Relaxation angepasst. Für diese Funktion treten sehr viele Parameter auf, so dass nicht davon ausgegangen werden kann, dass die Anpassung eindeutig ist. Da die Anpassung erst oberhalb der VFT-Temperatur physikalisch sinnvoll ist und vor allem die Hochtemperaturflanke gut repräsentieren sollte, wurden nur Daten oberhalb des ersten Knickpunkts verwendet $\left(\mathrm{ab} 100{ }^{\circ} \mathrm{C}\right)$. Die weiteren Startparameter wurden soweit möglich aus Literaturdaten gewonnen. Die Parameter $\tau_{0}$ und $\omega$ wurden festgehalten, da so die Zahl der freien Parameter etwas überschaubarer wird, $\tau_{0}$ unabhängig vom Messverfahren sein sollte und $\omega$ mit der Messfrequenz feststeht. Die Startwerte für die VFT-Parameter und erste Erwartungen an die Parameter $\alpha$ und $\gamma$ wurden von Bergman et al. [6] übernommen. Wie man in Abbildung 6.7 sieht, lässt sich eine hervorragend angepasste Funktion gewinnen. Die verwendeten Parameter sind in Tabelle 6.1 zusammengefasst.

\begin{tabular}{|c|c|c|c|c|c|c|}
\hline$\Delta E^{\prime \prime}$ & $\alpha$ & $\gamma$ & $\omega$ & $\tau_{0}$ & $D$ & $T_{\text {VFT }}$ \\
$40,9 \mathrm{GPa}$ & 0,342 & 0,0245 & $2 \pi \cdot 2450 \mathrm{~Hz}$ & $1,48 \cdot 10^{-11} \mathrm{~s}$ & 2,34 & 361 \\
\hline
\end{tabular}

Tabelle 6.1: Parameter für die angepasste Havriliak-Negami-Funktion aus Abb. 6.7

Betrachtet man diese, so ist die Relaxationsstärke $\Delta E^{\prime \prime}=E_{\infty}^{\prime \prime}-E_{s}^{\prime \prime}$ mit 40,9 GPa sehr viel größer als erwartet. Laut Bergman et al. [6] liegt der Parameter $\beta_{\mathrm{KWW}}$ für reines PMMA bei etwa 0,2. Damit erwartet man nach Alvarez et al. [1] einen HN-Parameter $\alpha$ von etwa 0,39. Dies ist sehr gut erfüllt. Allerdings ist zu bedenken, dass von mehreren Gruppen gefunden wurde, dass $\alpha$ zumindest für den $\beta$-Prozess temperaturabhängig ist [6, 107]. Auch wenn nicht auszuschließen ist, dass dies auch für den $\alpha$-Prozess der Fall ist, wurde dies hier nicht berücksichtigt, da hierfür keine Literaturdaten vorliegen und keine Informationen, die eine eventuelle Temperaturabhängigkeit beschreiben. Die Asymmetrie $\alpha$ wurde damit als einfacher, temperaturunabhängiger Anpassungsparameter verwendet.

Der HN-Parameter $\gamma$ ist mit 0,0245 sehr klein. Nach [1] würde man hier ebenfalls einen Wert von etwa 0,39 erwarten. Es ist jedoch zu bedenken, dass auch die Verbindungen zwi- 


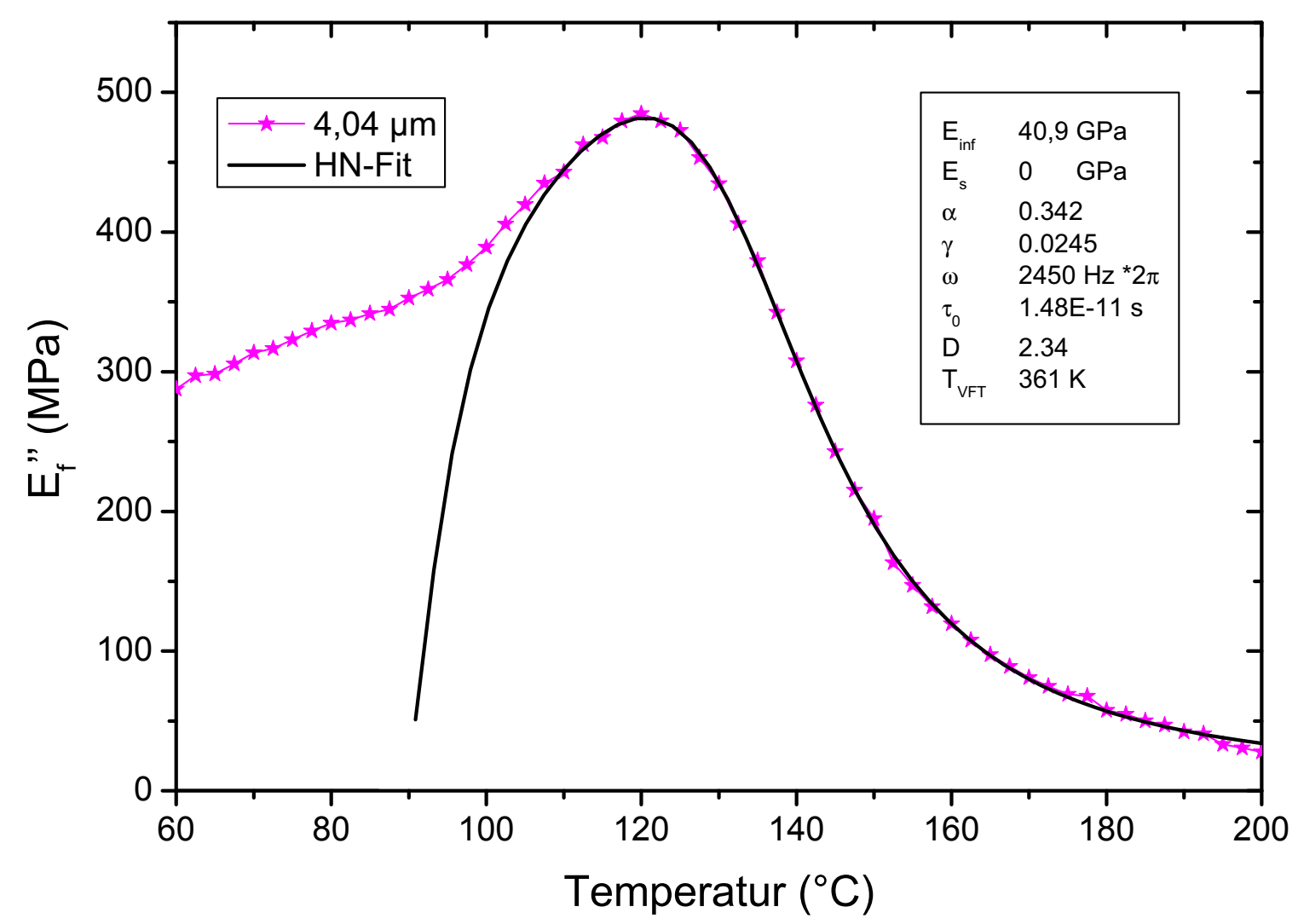

Abbildung 6.7: Berechneter Verlustmodul ( $\left.E^{\prime \prime}\right)$ für die Schicht mit 4,04 $\mu \mathrm{m}$ Dicke mit angepasster HN-Funktion (Imaginärteil, Gleichung 6.8)

schen dem KWW-Parameter $\beta_{\mathrm{KWW}}$ und den HN-Parametern, die in [1] behandelt werden, nicht eindeutig und nicht universell ist. Damit ist so ein geringer Wert durchaus möglich. Es kommt hinzu, dass das laserdeponierte PMMA im Vergleich zum Ausgangsmaterial verändert ist (siehe Kapitel 4). Damit ergibt sich die Möglichkeit, dass der stark verkleinerte Parameter $\gamma$ eine tatsächliche physikalische Bedeutung hat. Er ist dafür zuständig, die Asymmetrie in der HN-Funktion herzustellen. $\gamma=1$ bedeutet, dass die Funktion symmetrisch ist. Eine zufällige Verteilung an Relaxationsvorgängen und -Zeiten kann eine starke Asymmetrie hervorrufen.

Eine gute Übereinstimmung mit der Literatur ergibt sich für die Parameter der VogelFulcher-Tammann-Funktion. Für $D$ ist der Literaturwert 2,27 und für $T_{\mathrm{VFT}} 371 \mathrm{~K}$ zu finden [6]. Ein etwas niedrigeres $T_{\mathrm{VFT}}$ ist damit zu verbinden, dass die Glastemperatur für die stark verkürzten Ketten etwas verringert ist 83. $D$ ist fast identisch mit dem Literaturwert für reines PMMA, der geringfügig kleinere Wert deutet auf eine etwas größere Fragilität nach 
Angell [4] hin. Dies ist konsistent mit einer undefinierteren Zusammensetzung, durch die ein höherer Spielraum für die Energie der Bindungen vorhanden ist.

\subsection{Thermische Behandlung von PMMA}

Um Relaxationseffekte bei höheren Temperaturen zu messen, wurde die Probe aus Abschnitt 6.2 im folgenden zu immer höheren Endtemperaturen weit oberhalb von $T_{g}$ untersucht. Dabei wurden die Endtemperaturen 225, 250, 275, 300 und $350{ }^{\circ} \mathrm{C}$ gewählt. In diesem Abschnitt werden für alle Abbildungen volle Symbole für Aufheiz- und leere für Abkühlkurven verwendet. Betrachtet man zuerst die Resonanzfrequenzen in Abbildung 6.8, so sind in dieser Messung in den Messdaten (Inset) bei hohen Temperaturen Artefakte zu sehen, die vermutlich auf ein Rutschen des Substrats in der Einspannung zurückzuführen

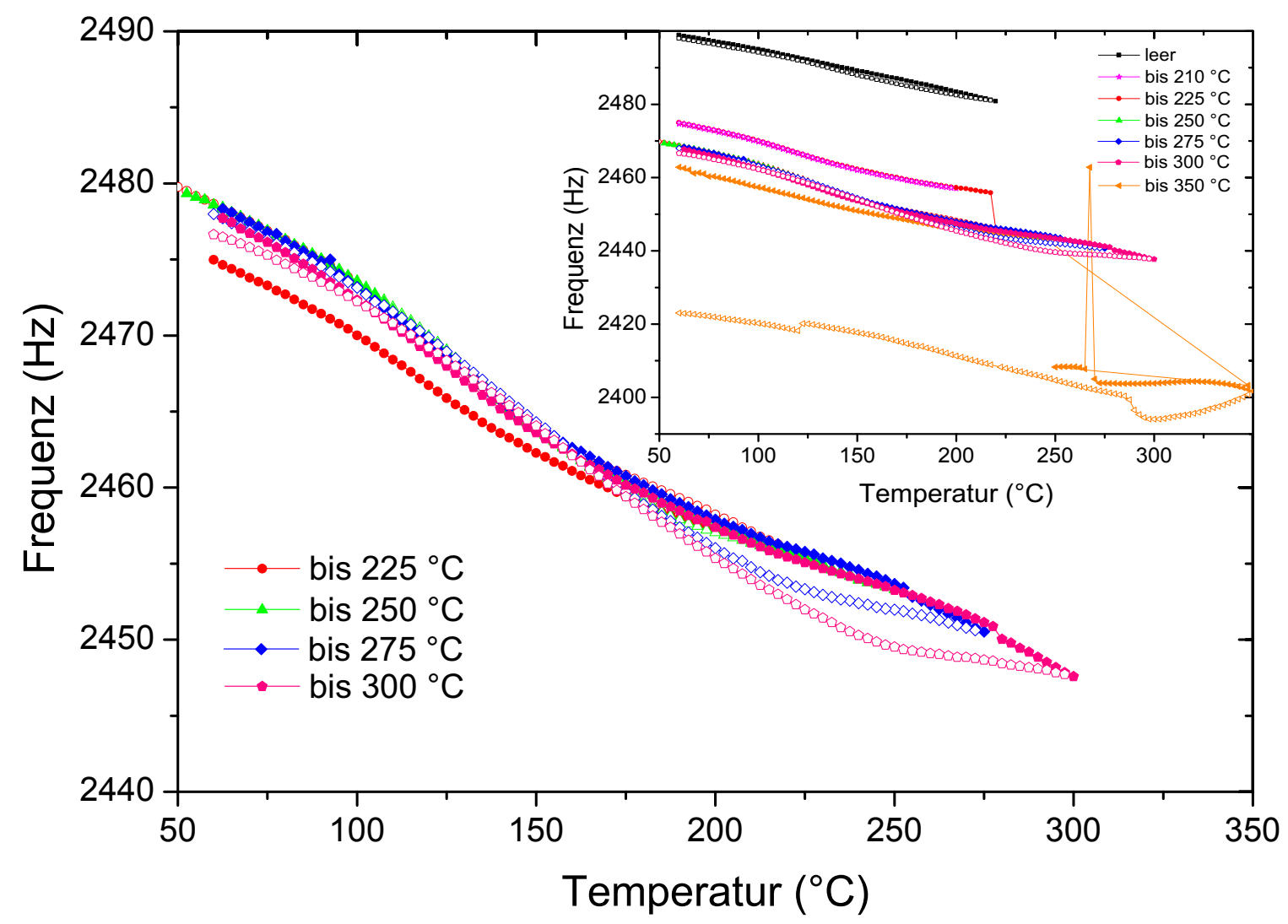

Abbildung 6.8: Resonanzfrequenz des PPXR gegen die Temperatur für mehrere Messungen mit verschiedener Endtemperatur. Inset: Originalmessdaten; Groß: korrigierte Daten (siehe Text) 
sind. Da, wenn diese einfache Erklärung zutrifft, dieser Effekt einfach durch Multiplikation mit einem Faktor korrigiert werden kann, sind im Hauptbild von Abbildung 6.8 die korrigierten Daten dargestellt.

Hier ist zu erkennen, dass schon nach der recht moderaten Auslagerung bei nur $225{ }^{\circ} \mathrm{C}$ die Resonanzfrequenz leicht ansteigt. Dies deutet auf einen Masseverlust hin. Vermutlich handelt es sich hierbei um kleine Moleküle, die in den laserdeponierten Filmen vorhanden sind. Für weitere Durchgänge bis hin zu $300{ }^{\circ} \mathrm{C}$ ist jedoch kein signifikanter Verlust an Masse mehr nachzuweisen. Die Hysterese bei höheren Temperaturen ist vermutlich auf die schlechtere Temperaturregelung in diesem Bereich zurück zu führen. Für die Auslagerung bei $350{ }^{\circ} \mathrm{C}$ ist eine völlige Veränderung festzustellen, hier wird der Film vermutlich völlig zerstört.

In Abbildung 6.9 sind die zu den obigen Frequenzmessungen gehörenden Dämpfungskurven für Auslagerungstemperaturen bis maximal $250{ }^{\circ} \mathrm{C}$ aufgetragen. Analog zur letzten

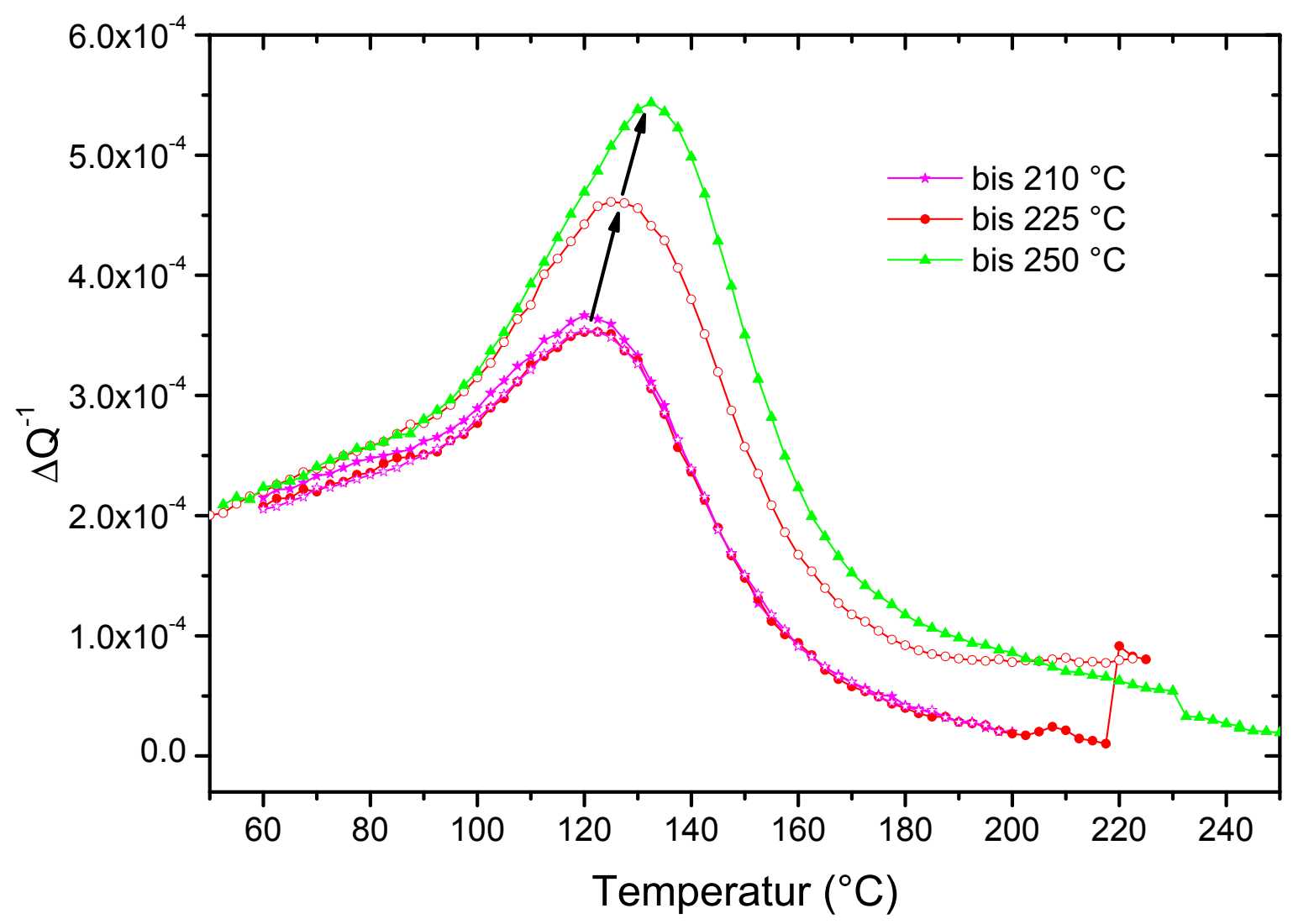

Abbildung 6.9: Mechanischer Verlust für Messungen mit unterschiedlicher Endtemperatur bis $250^{\circ} \mathrm{C}$ 
Messung in Abschnitt 6.2 treten bei der Messung bis $200{ }^{\circ} \mathrm{C}$ keine weiteren Effekte auf, die Probe wurde zuvor bei $210{ }^{\circ} \mathrm{C}$ ausgelagert. Bei der Messung bis $225^{\circ} \mathrm{C}$ tritt bei etwa $220{ }^{\circ} \mathrm{C}$ ein Sprung in der Dämpfung auf, der vermutlich auf das schon angeführte Rutschen des Substrats in der Halterung zurück zu führen ist, aber nicht so klar zu korrigieren ist wie der in der Frequenzkurve. Dennoch ist nicht zu bezweifeln, dass im darauf folgenden Kühlzyklus die Dämpfung signifikant höher ist, als beim zuvor erfolgten Heizen. Dieses Verhalten wird i. A. mit der Relaxation des Materials bei höheren Temperaturen erklärt, durch welche freies Volumen ausgeheilt und damit die Effizienz der Energiedissipation erhöht wird. Dies wurde auch für laserdeponierte Proben schon früher mit dem Doppelpaddeloszillator beobachtet [96]. Da aus den Frequenzmessungen gleichzeitig geschlossen werden kann, dass Material verloren geht, ist zu vermuten, dass gerade kleine Moleküle abdampfen, ohne dass die polymere Struktur beschädigt wird.

Beim darauf folgenden Heizzyklus ist dieser Trend noch einmal verstärkt. Das Maximum steigt weiter an und wandert zu noch höheren Temperaturen. Da die Probe zwischenzeitlich kalt war, ist das Verhalten hier nicht einfach durch Relaxation zu erklären und bisher noch unverstanden.

Die Dämpfung für die weiteren Auslagerungszyklen ist in Abbildung 6.10 gezeigt. Hier ist $\mathrm{zu}$ sehen, dass die erste Abkühlkurve nach der Auslagerung auf $250{ }^{\circ} \mathrm{C}$ wieder genau auf der vorherigen Abkühlkurve bis $220^{\circ} \mathrm{C}$ liegt. Dies legt nahe, dass bei dieser Auslagerung keine weitere Veränderung durch die Auslagerung aufgetreten ist. Für alle weiteren Auslagerungsschritte fällt das Maximum kontinuierlich ab, ohne seine Position auf der Temperaturachse zu verändern. Da kein weiteres Material abdampft, liegt die Vermutung nahe, dass die polymere Struktur ohne größeren Massevelust verloren geht, z. B. durch Quervernetzung über Doppelbindungen oder höchstens den geringfügigen Verlust von Seitengruppen.

Gleichzeitig ist zu sehen, dass für alle Auslagerungsschritte der erste Knick, der mit der Glastemperatur assoziiert wird, seine Position nicht verändert. Dies bedeutet, dass zwar die Glastemperatur identisch bleibt, die Dynamik, die knapp oberhalb davon auftritt jedoch zumindest leicht verändert wird.

Nach Auslagerung auf $350{ }^{\circ} \mathrm{C}$ ist die Dämpfung völlig verschwunden. Da nach Manring [76] die Zersetzung der PMMA-Hauptkette wesentlich zwischen 250 und $350{ }^{\circ} \mathrm{C}$ erfolgt, ist zu vermuten, dass hier das Polymer völlig zerstört wurde. Es könnte abdampfen oder Reste als Kohlenstoffverbindung oder verbunden mit Silizium oder Silber auf der Oberfläche zurückbleiben. Nach der Messung ist auf dem Substrat noch Film zu sehen, aufgrund 


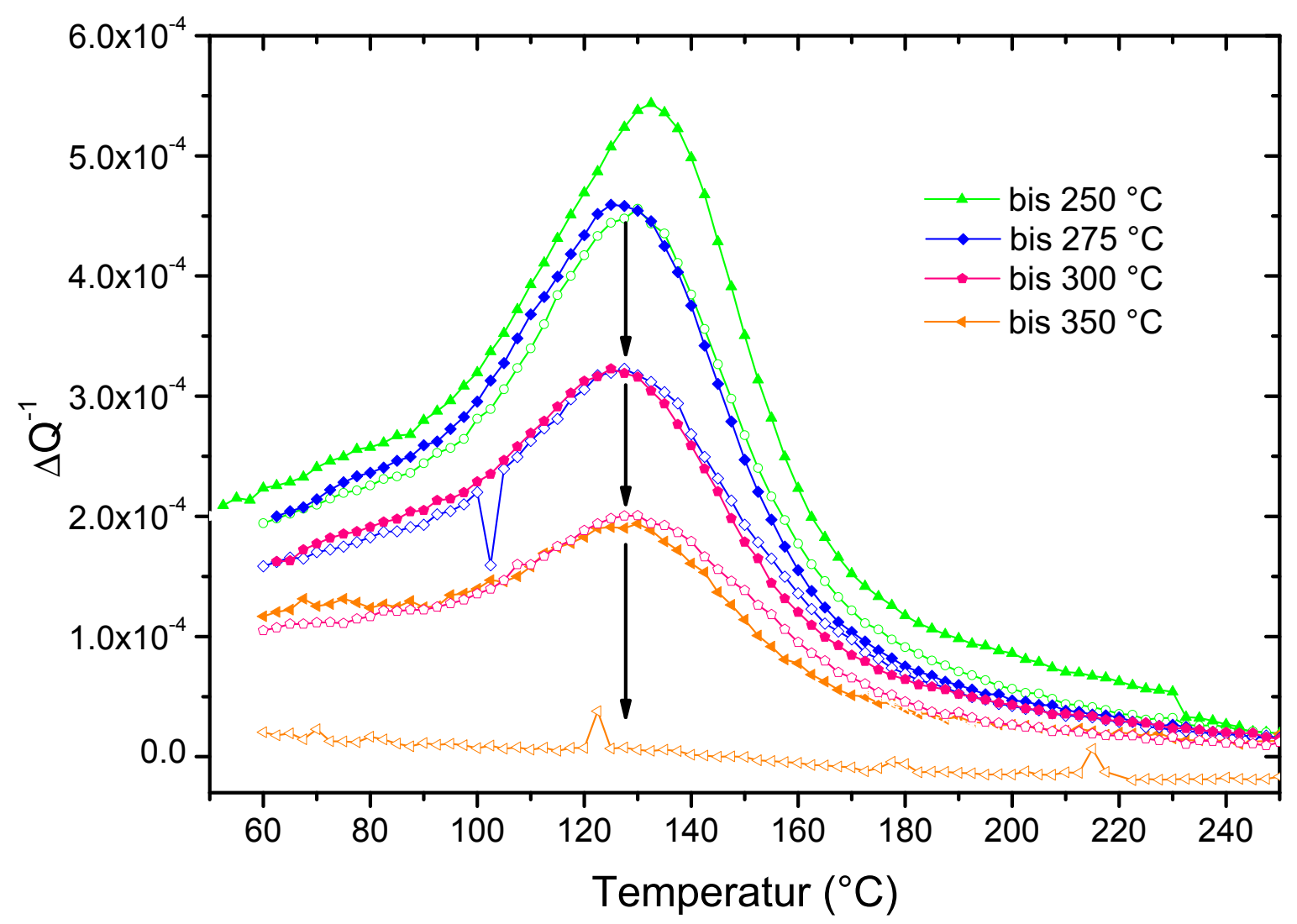

Abbildung 6.10: Mechanischer Verlust für Messungen mit unterschiedlicher Endtemperatur bis $350^{\circ} \mathrm{C}$

der geringen Größe des bedampften Substrats ist jedoch nicht zu messen, um was es sich handelt.

\subsection{Vergleich spektroskopischer Methoden}

Für eine neue Methode wie PPXR ist es wichtig, zu entscheiden, wie sie im Vergleich mit herkömmlichen, etablierten Untersuchungsmethoden zu sehen ist. Von laserdeponierten Proben wurden Spektroskopiedaten bisher mittels des Doppelpaddeloszillators und der dielektrischen Spektroskopie gewonnen.

Der Doppelpaddeloszillator (DPO) misst den Schermodul des Films bei der festen Frequenz von 5,4 kHz. Durch die geringe Grunddämpfung der gewählten Schwingungsmode ist die Messung sehr präzise und bis zu geringen Schichtdicken hin durchführbar. Die DPO- 
Messungen wurden in Zusammenarbeit von Peter Rösner im 1. Physikalischen Institut, Göttingen, durchgeführt [97, 96].

Die Standardmethode für die Spektroskopie an Polymeren ist die dielektrische Spektroskopie. Hierfür ist bekannt, dass bei PMMA vor allem die $\beta$-Relaxation angeregt wird [105], da nur diese ein Dipolmoment hat. In diesem Fall wurden die laserdeponierten Schichten am Lehrstuhl für Experimentalphysik V der Universität Augsburg gemessen. Es wurden temperaturabhängige Messungen bei $5,4 \mathrm{kHz}$ durchgeführt, um sie mit den DPO Messungen zu vergleichen, und bei $540 \mathrm{~Hz}$, einer Frequenz, bei der $\alpha$ - und $\beta$-Relaxtion noch trennbar sind (Beginn des mergings). Für 5,4 kHz sind beide Peaks untrennbar zu einem verschmolzen, wobei, wenn man von einer einfachen Superposition ausgeht, davon ausgegangen werden kann, dass der $\beta$-Prozess deutlich dominiert.

In Abbildung 6.11 sind die Messungen an vergleichbaren, bei Raumtemperatur laserdeponierten Proben für alle drei hier aufgeführten Methoden zusammen aufgetragen. Der

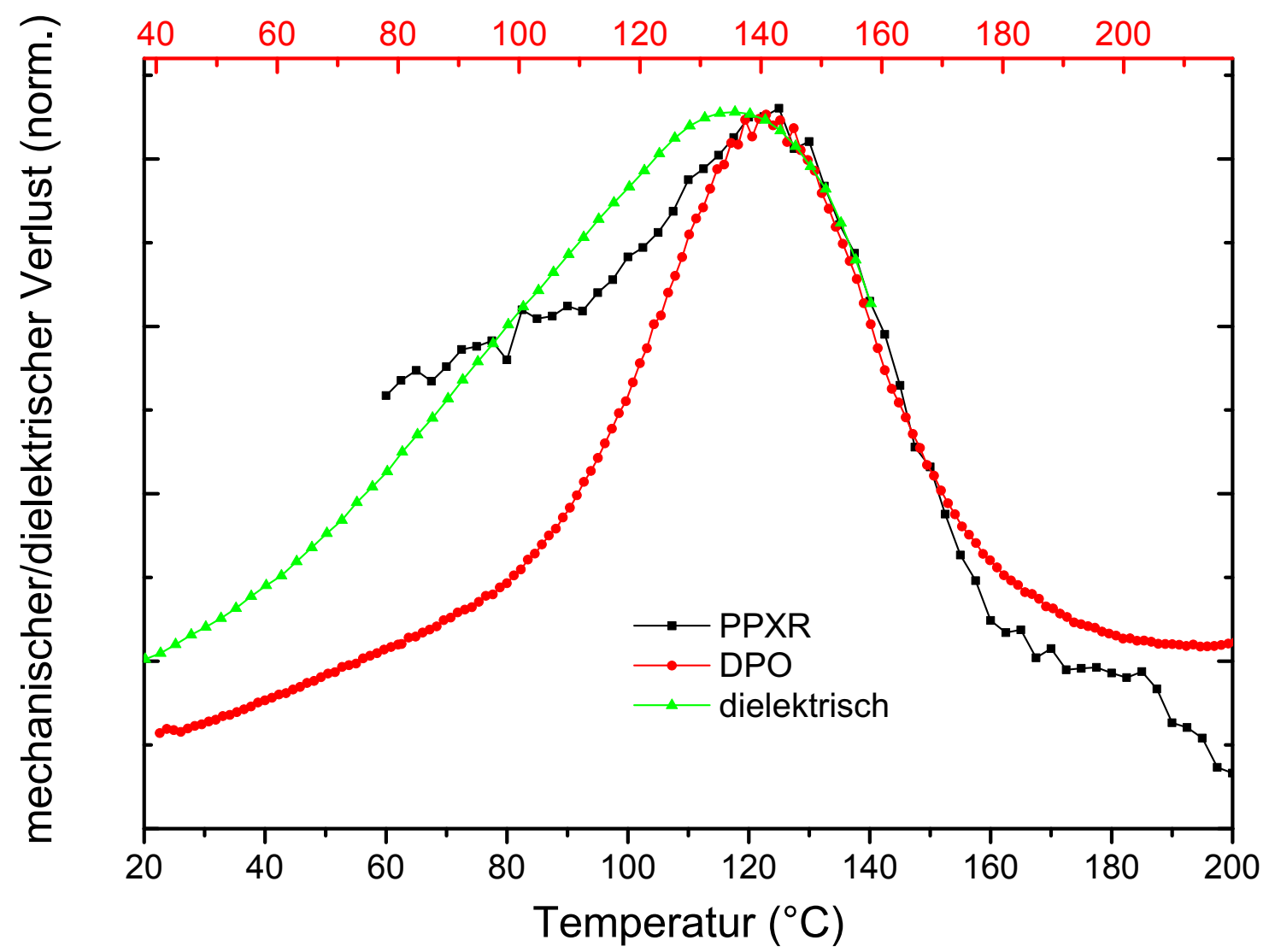

Abbildung 6.11: Vergleich der verschiedenen spektroskopischen Methoden für bei Raumtemperatur laserdeponiertes PMMA (Skala oben für DPO, siehe Text) 
Vergleich über absolute Temperaturen ist schwierig, da die Bestimmung besonders im Fall der mechanischen Spektroskopie experimentelle Unsicherheiten mit sich bringt. Aus diesem Grund wurde hier die dielektrische Spektroskopie als Maßstab genommen und es stellt sich heraus, dass diese mit der des PPXR gut übereinstimmt. Das Maximum der DPO-Messung ist um $18{ }^{\circ} \mathrm{C}$ zu hohen Temperaturen verschoben, weshalb hierfür die Temperaturskala verschoben wurde.

Die Messung für PPXR wurde so ausgewählt, dass die Proben möglichst gut vergleichbar sind, alle wurden bei Raumtemperatur hergestellt, die Messfrequenz ist jedoch unterschiedlich, sie liegt bei nur 2,6 kHz. Damit sollte die Temperaturskala um etwa $5-7{ }^{\circ} \mathrm{C}$ verschoben sein. Die Frequenz sollte jedoch deutlich im merging-Bereich liegen (siehe Abschnitt 6.1).

Schiebt man die Kurven in dieser Art aufeinander, so sieht man, dass für die beiden mechanischen Methoden vor allem die Hochtemperaturflanke sehr gut übereinstimmt. Auf der Tieftemperaturseite weicht die PPXR-Messung bei Temperaturen unterhalb von $110{ }^{\circ} \mathrm{C}$ zu höheren Dämpfungen ab und erreicht einen linearen Anstieg, der oberhalb desjenigen des DPO liegt. Der Grund hierfür liegt vermutlich darin, dass unterschiedliche Messgrößen betrachtet werden. Während mit PPXR hauptsächlich der E-Modul bestimmt wird, misst der DPO den Schermodul des Films. Diese beiden Größen sind durch die Poissonzahl $\nu$ miteinander verbunden, so dass gilt

$$
E=2 G(1+\nu)
$$

Da es sich bei den in Abbildung 6.11 aufgetragenen Größen jeweils um den Imaginärteil der komplexen Größe handelt, müssen wir hier eine komplexe Poissonzahl betrachten, was in der Literatur nur selten geschieht. Für den Realteil wurde eine Temperaturabhängigkeit für PMMA bestimmt, die zeigt, dass die Poissonzahl bei steigender Temperatur im Intervall von -20 bis $+125^{\circ} \mathrm{C}$ bei $10^{-2}-10^{4} \mathrm{~Hz}$ steigt [58, 75]. Vergleicht man dies mit Ergebnissen von Lakes [69], wo Real- und Imaginärteil von $\nu$ meist eine gegenläufige Tendenz haben, so scheint es möglich, dass die Differenzen hieraus zu erklären sind. Eine absolute Skala für die Poissonzahl lässt sich daraus aber nicht berechnen, da die hier gezeigten Kurven normierte Dämpfungskurven sind und sowohl die Verfahren als auch die Schichten zu unterschiedlich sind, um einen quantitativen Vergleich zu wagen. Ignoriert man dies und auch die große Unsicherheit durch die Korrektur der Temperatur, so erhält man für die Umrechnung der beiden Kurven ineinander eine linear abfallende Poissonzahl im Bereich von 60 bis $110{ }^{\circ} \mathrm{C}$, danach bleibt der Wert konstant. Für höhere Temperaturen gleicht das mecha- 
nische Verhalten der Schicht immer mehr dem einer Flüssigkeit, d. h. die Poissonzahl sollte gegen einen konstanten Wert laufen. Die größten Veränderungen liegen erwartungsgemäß im Bereich des Glasübergangs.

Deutliche Unterschiede zeigen sich dagegen im Vergleich zu den Messungen der dielektrischen Spektroskopie. Diese passen (ohne Temperaturverschiebung) ebenfalls an der Hochtemperaturflanke hervorragend überein, sowohl das Maximum als auch die Tieftemperaturflanke liegen jedoch bei niedrigeren Temperaturen. Dies kann damit begründet werden, dass das dielektrische Dämpfungsmaximum hier durch die $\beta$-Relaxation dominiert wird und sich damit bei etwas tieferen Temperaturen befindet. Auf die Hochtemperaturflanke wirkt sich die $\alpha$-Relaxation jedoch auch in dielektrischen Messungen immer noch stark aus. Misst man nun mit der mechanischen Spektroskopie, so sollte im Wesentlichen die $\alpha$-Relaxation vermessen werden, da eine Anregung über mechanische Verformung erfolgt und die $\beta$-Relaxation keinen Beitrag dazu liefert. Damit ist es konsistent, dass die Form an der Hochtemperaturflanke passt. Dagegen zeigen die Messungen von Muzeau et al. [82, dass dielektrische und mechanische Spektroskopie die gleichen Ergebnisse zeigen. Allerdings wurden die dort verglichenen Daten für Temperaturen deutlich unterhalb von $T_{g}$ gemessen $\left(-20^{\circ} \mathrm{C}\right)$, und aus diesen Datenpunkten ist eine Extrapolation auf den kritischen Bereich kaum möglich.

Zur Bestätigung der hier aufgestellten Hypothese sollen die dielektrischen Messung bei $540 \mathrm{~Hz}$ mit PPXR-Messungen verglichen werden. Das hierfür benötigte PPXR-Substrat ist etwa $16 \mathrm{~mm}$ lang, bei 1-2 mm Breite ist die präzise Präparation dementsprechend schwierig und die Messung, die in Abbildung 6.12 unten gezeigt ist, zeigt stärkeres Rauschen, aber eine Frequenz von $520 \mathrm{~Hz}$. Diese Variabilität ist eine große Stärke der PPXR gegenüber anderen mechanischen Spektroskopiemethoden. Zum Vergleich ist im oberen Teil der Abbildung eine PPXR-Messung bei 2,6 kHz zu sehen. Es zeigt sich, dass sowohl die Form der Kurve als auch die Position des Maximums im Rahmen der Ablesbarkeit von dieser Kurve nicht signifikant verändert werden.

Betrachtet man nun im Vergleich dazu die dielektrische Spektroskopie bei $540 \mathrm{~Hz}$, wie es in Abbildung 6.13 gezeigt ist, so erkennt man hier (rote Kurve) deutlich, dass zwei Peaks auftreten, auch wenn diese sich überschneiden (Beginn des mergings). Der linke davon gehört zur $\beta$-, der rechte zur $\alpha$-Relaxation. Würde also bei der mechanischen Spektroskopie das gleiche Verhältnis der beiden Prozesse angeregt, müsste sich die PPXR-Kurve deutlich verschieben. Passt man nun an den $\beta$-Prozess der dielektrischen Messung eine Havriliak-Negami-Funktion (Gleichung 6.8) an und zieht diese von der Messung ab, so er- 


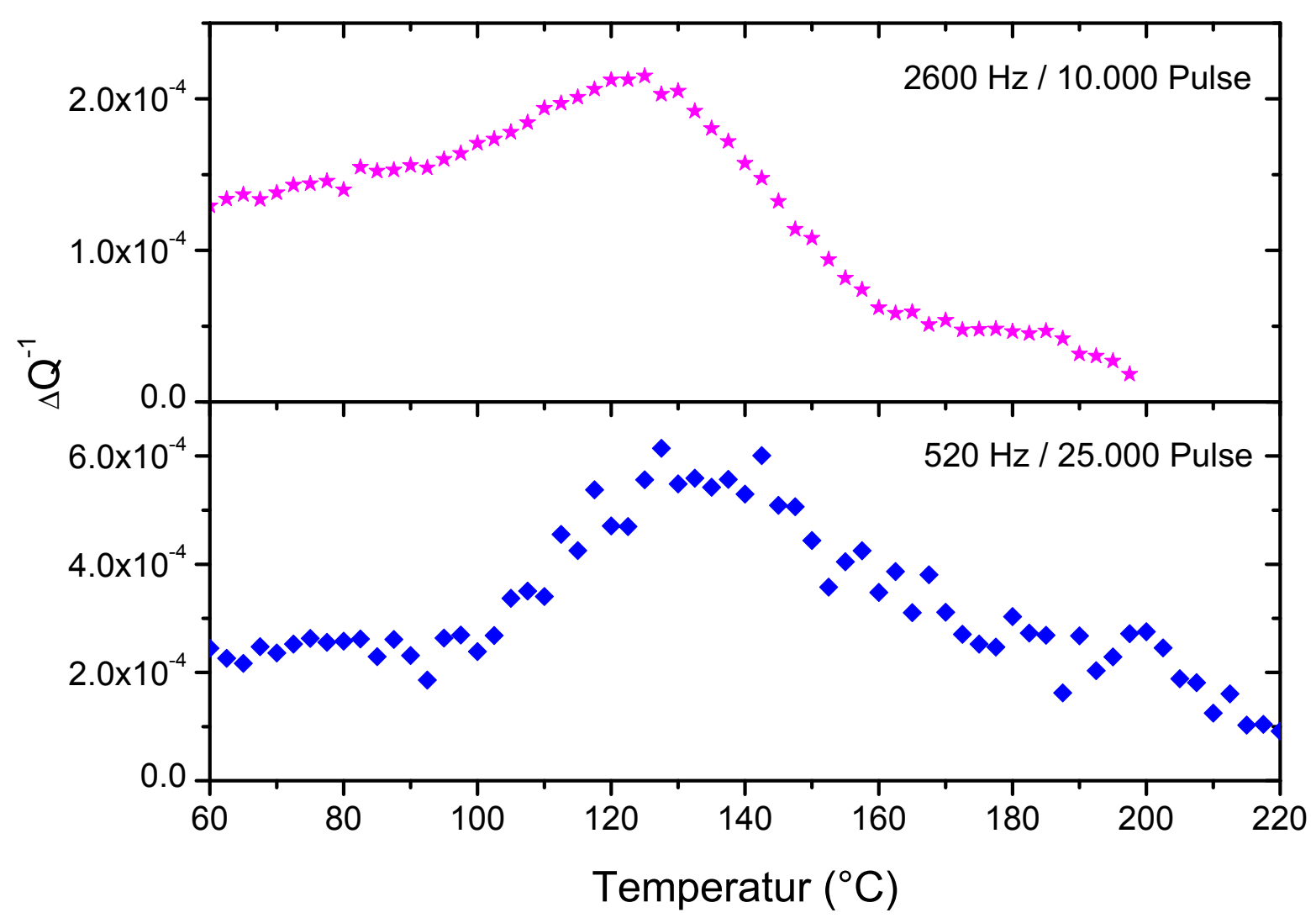

Abbildung 6.12: Vergleich von PPXR-Messungen von bei Raumtemperatur deponierten PMMA Proben mit $2600 \mathrm{~Hz}$ und $520 \mathrm{~Hz}$

gibt sich die schwarz gezeichnete Kurve. Diese sollte nun im Wesentlichen den Anteil der $\alpha$-Relaxation enthalten. Wie man sieht, passt diese zumindest Qualitativ sehr gut zu den mit PPXR erhaltenen Messwerten bei $520 \mathrm{~Hz}$. Es ist zu bedenken, dass die Anpassung der HN-Funktion in die teilweise überlappende Funktion eine große Variationsbreite für die Parameter offen lässt, die hier gezeigte Funktion soll also nicht eine eindeutige Lösung bilden, sondern nur einen Versuch darstellen, die ungefähre Form und Lage der $\alpha$-Relaxation in der dielektrischen Messung zu erhalten. Die Ergebnisse sollen aus diesem Grund nur qualitativ verglichen werden.

Es bleibt festzustellen, dass die mechanischen Spektroskopiemethoden ähnliche Ergebnisse liefern. Außer im Tieftemperaturbereich, der für PPXR durch die Messung des Elastizitäts- anstelle des Schermoduls, also einer anderen Größe, einen deutlich höheren Verlauf zeigt als für den DPO, stimmen die Kurven gut überein. Der Unterschied liegt in den spe- 


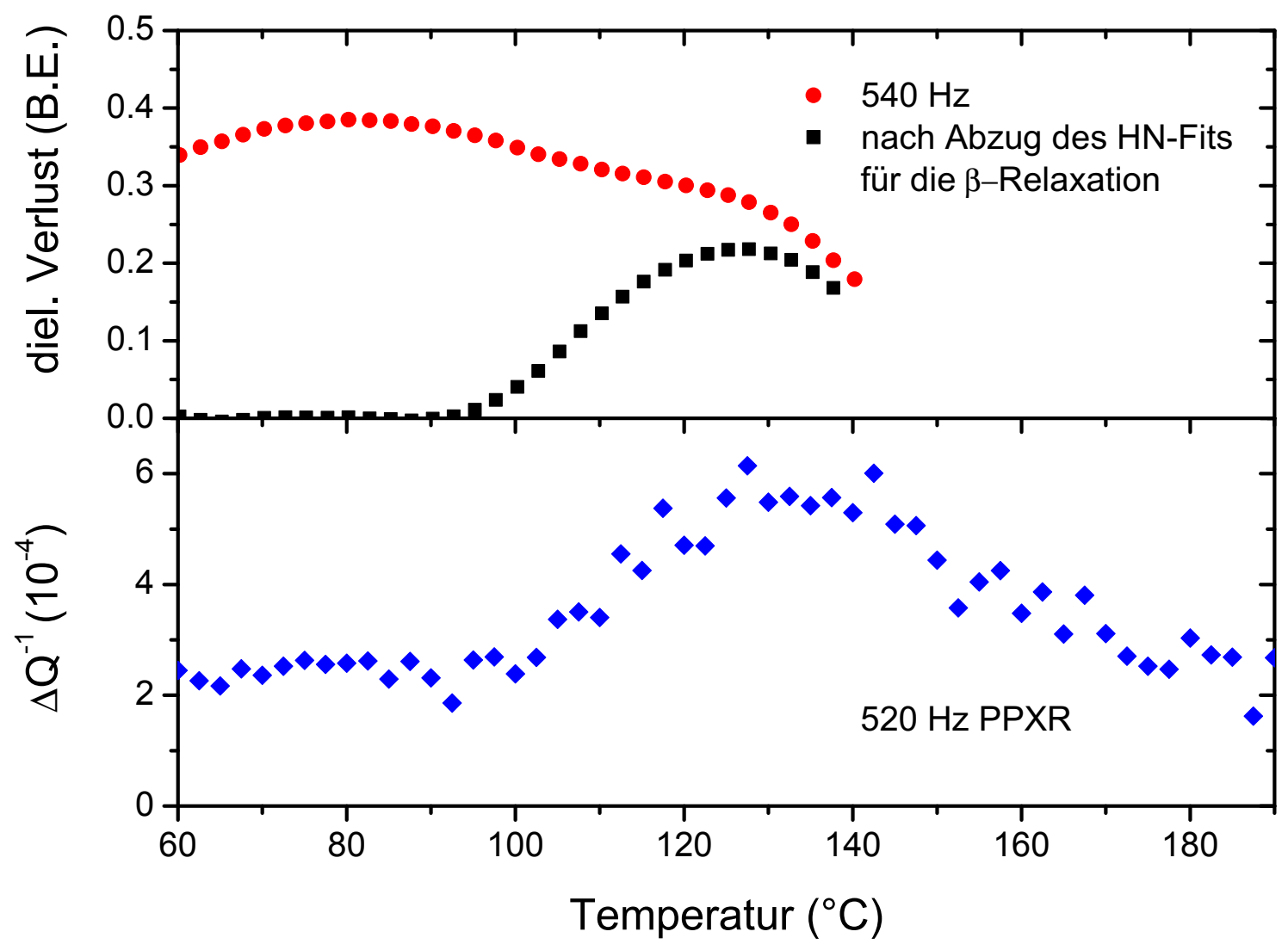

Abbildung 6.13: Vergleich der PPXR-Messung bei $520 \mathrm{~Hz}$ mit dielektrischer Spektroskopie bei $540 \mathrm{~Hz}$. Für die schwarze Kurve wurden an den $\beta$-Peak eine Havriliak-NegamiFunktion angefittet und abgezogen, so dass nur die $\alpha$-Relaxation übrig bleibt.

zifischen Stärken der beiden Methoden, dem sehr geringen Untergrund des DPO und der hohen Flexibilität und der in-situ Nutzbarkeit für PPXR.

Die dielektrische Spektroskopie regt dagegen hauptsächlich die für die Bestimmung mechanischer Eigenschaften unwichtigere $\beta$-Relaxation an und kann damit für Proben bei denen nur Seitenketten polar sind, nur begrenzt zur Bestimmung der mikroskopischen Prozesse der makroskopischen mechanischen Eigenschaften herangezogen werden. Bei komplett unpolaren Systemen fällt diese Methode damit völlig aus. 


\subsection{Abhängigkeit der Messung von der Resonanzfre- quenz}

Wie in Abschnitt 6.1 erläutert, hängt die Temperaturabhängigkeit der mechanischen Eigenschaften stark von der betrachteten Frequenz ab. Mittels der vibrating Reed Technik war es in dieser Arbeit möglich, die Frequenz im Bereich zwischen 500 und $7.500 \mathrm{~Hz} \mathrm{zu}$ variieren.

In Abbildung 6.14 sind Messungen bei unterschiedlicher Frequenz zwischen 1,6 und $7,5 \mathrm{kHz}$ dargestellt. Um die Bedingungen möglichst vergleichbar zu halten, wurden Proben

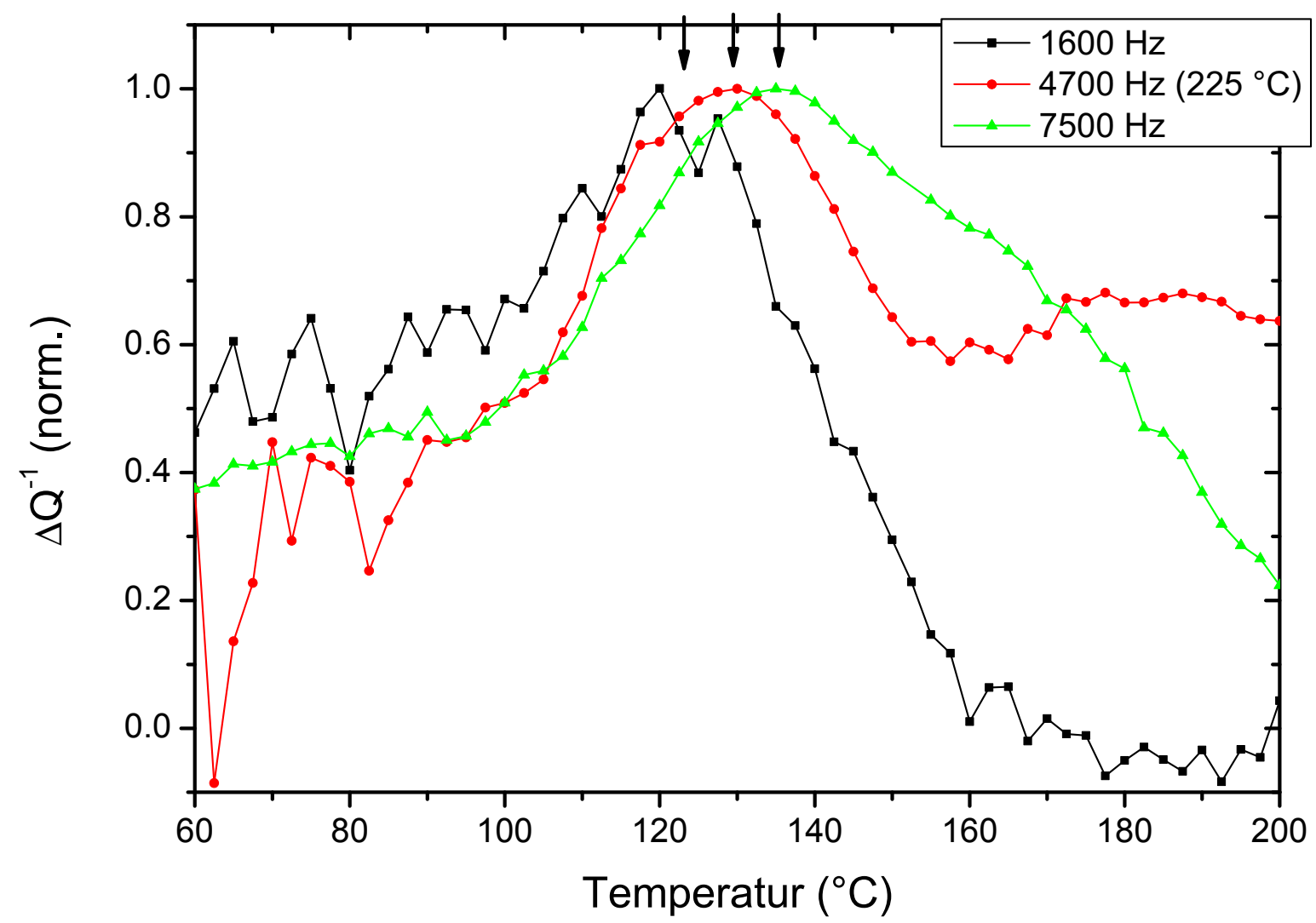

Abbildung 6.14: Vergleich von PPXR-Messungen bei verschiedenen Resonanzfrequenzen. Die Proben wurden bei $200{ }^{\circ} \mathrm{C}$ hergestellt, die Probe für $4700 \mathrm{~Hz}$ bei $225^{\circ} \mathrm{C}$.

mit $200{ }^{\circ} \mathrm{C}$ Substrattemperatur während der Herstellung ausgewählt. Nur die mittlere Probe bei $4,7 \mathrm{kHz}$ wurde bei $225^{\circ} \mathrm{C}$ hergestellt. Die Schichten waren unterschiedlich dick und die Messungen wurden normiert, die absolute Höhe kann daher etwas abweichen. Es ist zu sehen, dass die Messungen im Bereich unterhalb der Glastemperatur einen parallelen 
Verlauf haben. Auch der Knickpunkt, der mit der Glastemperatur verbunden ist, verschiebt sich nur leicht zu höheren Temperaturen. Hier hat die Messfrequenz also wenig Einfluss auf den Verlauf der Dämpfung. Die Daten sind in Tabelle 6.2 zusammengefasst.

\begin{tabular}{|c|c|r|c|}
\hline Messfrequenz & Substrattemperatur & Knickpunkt & Maximum \\
$1600 \mathrm{~Hz}$ & $200{ }^{\circ} \mathrm{C}$ & $99(5){ }^{\circ} \mathrm{C}$ & $123(5){ }^{\circ} \mathrm{C}$ \\
$4700 \mathrm{~Hz}$ & $225{ }^{\circ} \mathrm{C}$ & $104(3){ }^{\circ} \mathrm{C}$ & $130(3){ }^{\circ} \mathrm{C}$ \\
$7500 \mathrm{~Hz}$ & $200{ }^{\circ} \mathrm{C}$ & $104(5){ }^{\circ} \mathrm{C}$ & $136(4){ }^{\circ} \mathrm{C}$ \\
\hline
\end{tabular}

Tabelle 6.2: Daten der Messkurven aus Abb. 6.14 für den Vergleich unterschiedlicher Messfrequenzen

Wie zu erwarten, nimmt der Einfluss der Messfrequenz im Bereich und oberhalb der Glastemperatur bedeutend zu. Die Temperatur des Maximums verschiebt sich mit zunehmender Frequenz zu höheren Temperaturen. Unterschiede zeigen sich auch in der Kurvenform für höhere Temperaturen. Die Abweichung für $4700 \mathrm{~Hz}$ kann an der unterschiedlichen Probenpräparation liegen und wird in Abschnitt 6.6 diskutiert. Für die Kurve bei $7500 \mathrm{~Hz}$ tritt eine Schulter im Bereich um $160{ }^{\circ} \mathrm{C}$ auf, deren Herkunft hier ungeklärt bleiben muss.

\subsection{Modifikation der Filme durch die Substrattempe- ratur}

Wie aus Kapitel 4 bekannt ist, ist die einzige bekannte Methode, die Filmeigenschaften deutlich in Richtung des reinen PMMA zu verändern ohne das Target zu modifizieren, die Veränderung der Substrattemperatur. Hierdurch wird nicht nur, wie beim Auslagern, die Oberflächenmorphologie [120] sondern auch die Kettenlänge des Filmmaterials deutlich verändert [119, 118]. Die Abhängigkeit der mechanischen Relaxation von dieser Veränderung soll in diesem Abschnitt untersucht werden.

Es wurden bei Raumtemperatur und zwischen 150 und $225{ }^{\circ} \mathrm{C}$ hergestellte Filme in Abständen von $25{ }^{\circ} \mathrm{C}$ untersucht. Die Schichtdicke ist für alle Kurven unterschiedlich, sie wurden deshalb auf die maximale Dämpfung normiert. Dies zeigt Abbildung 6.15.

Wiederum ist zu sehen, dass die Kurven im Bereich deutlich unterhalb der Glastemperatur im Rahmen der Messgenauigkeit parallel verlaufen. Ein Effekt für eine unterschiedliche Höhe könnte beispielsweise durch eine unterschiedlich starke Auslenkung begründet sein. Laut Koppelmann et. al. [58] hängt der E-Modul geringfügig von der angelegten Spannung ab. Allerdings beträgt dieser Effekt weniger als 10\%, also nicht genug um diese Differenzen 


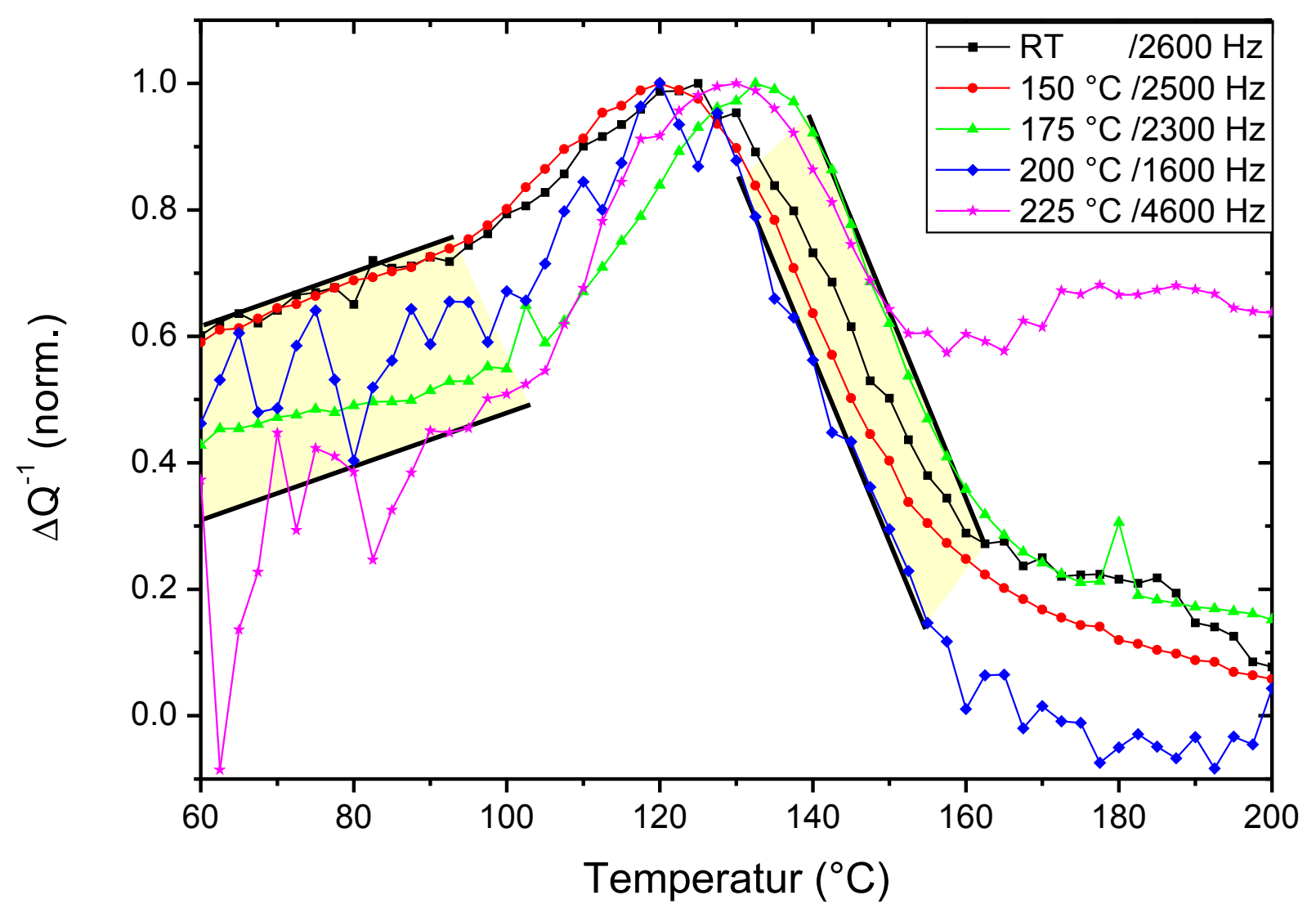

Abbildung 6.15: Vergleich von PPXR-Messungen für laserdeponierte Proben, die bei verschiedenen Substrattemperaturen hergestellt wurden.

zu erklären. Vermutlich liegt die Ursache in den deutlich unterschiedlichen Schichtdicken, die von der einfachen Normierung auf das Maximum nicht erfasst werden. Zusätzlich zeigen die verschiedenen Messungen eine deutlich unterschiedliche Qualität und die Fehler durch Rauschen werden durch die Normierung verstärkt.

Weiterhin sind die Kurven der bei Raumtemperatur und bei $150{ }^{\circ} \mathrm{C}$ hergestellten Probe im Rahmen der Messgenauigkeit von $2,5^{\circ} \mathrm{C}$ identisch, die erwartete Veränderung der Kettenlänge macht sich also nicht bemerkbar. Ein lineares Wachstum vorausgesetzt, erwartet man nach den Ergebnissen von O'Driscoll und Amin Sanayei [83] auch nur eine Veränderung von ca. $0,5^{\circ} \mathrm{C}$ was unterhalb der hier möglichen Auflösung liegt. Ein deutlicher Unterschied zeigt sich für die Kurve des bei $175{ }^{\circ} \mathrm{C}$ deponierten Films, die um $15{ }^{\circ} \mathrm{C}$ zu höheren Temperaturen verschoben ist. Da die Kurve kaum Rauschen zeigt und keine Erkenntnisse für einen Messfehler vorliegen, ist dies momentan nicht zu erklären. Weiterhin 
liegt die darauf folgende Kurve für $200{ }^{\circ} \mathrm{C}$ Herstellungstemperatur wieder bei den vorherigen, was für die bei $175{ }^{\circ} \mathrm{C}$ hergestellten einen nicht identifizierbaren Fehler vermuten lässt.

Klare Abweichungen zeigen die Messungen für die bei $225{ }^{\circ} \mathrm{C}$ hergestellte Probe, deren Maximum einerseits um ca. 7 Grad verschoben ist, was vermutlich an der höheren Messfrequenz liegt (vgl. Abschnitt 6.5), andererseits fällt die Dämpfung für hohe Temperaturen nicht gegen Null. Dies ist vermutlich mit der schon deutlich veränderten Struktur zu erklären. Wie schon in Abschnitt 6.3 gezeigt wurde, treten bei dieser Temperatur vermutlich schon weitere Veränderungen, die mit Masseverlust im Film verbunden sind, auf. Wenn es sich dabei nicht nur um das Abdampfen kleinerer Moleküle, sondern um eine teilweise Abspaltung von kleineren Bestandteilen handelt, so werden gerade während der Deposition, wo die Konzentration an reaktiven Molekülen sehr hoch ist, Veränderungen chemischer Bindungsverhältnisse eintreten, die das Material stark beeinflussen. Durch einen höheren Grad an Vernetzung beispielsweise, würde das Material von einem Thermoplasten in Richtung eines Duroplasten verändert und damit bei höheren Temperaturen nicht mehr flüssig werden.

Mit Vorbehalt der Unsicherheit dieser Messung lassen sich daraus Rückschlüsse auf den Mechanismus des Kettenlängenwachstums bei höherer Substrattemperatur gewinnen. Es konnte schon in Kapitel 4 gezeigt werden, dass die mittlere Kettenlänge mit der Temperatur zunimmt und das Wachstum mit einer Energiebarriere von $0,2 \mathrm{eV}$ verbunden ist. Diese Energie ist zu niedrig, um existierende chemische Bindungen zu brechen. Das Wachstum muss also durch aktivierte Zustände während der Deposition zustande kommen, was konsistent damit ist, dass durch Auslagerung keine Veränderungen der Kettenlänge auftreten [120]. Allerdings scheint es sich nach den Erkenntnissen dieses Abschnitts nicht um einen einfachen Zusammenschluss der Hauptkette, also eine Reparatur der Zerstörung durch den Laserbeschuss zu handeln, sondern vielmehr um eine eher zufällige Vernetzung. Ein linearer Zusammenschluss der Ketten würde nicht zu einem Duroplasten führen. Sehr hoch vernetzte Anteile, wie sie hierfür nötig wären, sind unlöslich und mittels SEC nicht erkennbar. Anteile, die fast linear verbunden sind, werden darin allerdings einfach als größere Ketten erkannt.

Diese Ergebnisse sind konsistent mit Süske [117], wo gezeigt wird, dass die mechanischen Eigenschaften, gemessen im quasistatischen Fall eines Härteeindrucks mit einem Nanoindenter, bei einer Substrattemperatur oberhalb von $200^{\circ} \mathrm{C}$ verändert werden. Der reduzierte E-Modul steigt hier stark an, die chemischen Bindungsverhältnisse, die im Material auftre- 
ten verändern sich, indem der Anteil der $\mathrm{C}=\mathrm{O}$ Bindung im Vergleich zur $\mathrm{C}-\mathrm{H}$ Bindungen systematisch zurückgeht. Auch dies spricht für eine Vernetzung, die ohne Masseverlust vor allem über die Carbonylbindung erfolgt. Vergleichbar ist ebenfalls, dass das nachträgliche Auslagern sehr viel höhere Temperaturen erfordert, um deutliche Änderungen zu erreichen.

Zusammenfassend ist festzustellen, dass nur eine veränderte Kettenlänge in dem hier betrachteten Bereich keine Änderung des temperaturabhängigen mechanischen Verlusts hervorruft, die für die hier dargestellten Messungen signifikant wäre. Allerdings lässt die bei einer Substrattemperatur von von $225{ }^{\circ} \mathrm{C}$ hergestellten Probe während der Herstellung darauf schließen, dass das Kettenwachstum keineswegs linear, sondern durch zufällige Vernetzung geschieht, wodurch bei hohen Temperaturen starke Eigenschaftsänderungen auftreten. 


\section{Zusammenfassung}

Als Ausgangspunkt dieser Arbeit war aus Vorarbeiten bekannt, dass bei der gepulsten Laserdeposition von Poly(methyl methacrylat) (PMMA) zweikomponentige Filme deponiert werden, die aus einer glatten, stark vernetzten Komponente und Tröpfchen aus PMMA mit stark reduzierter Kettenlänge bestehen. Ziel dieser Arbeit war es, das Bild des Mechanismus der Laserablation und -Deposition von Polymeren zu erweitern, und zu ermitteln wie dieser Mechanismus mit dem Ziel veränderter Eigenschaften der deponierten Filme modifiziert werden kann. Ein besonderes Augenmerk wurde dabei auf die frequenz- und temperaturabhängigen mechanischen Eigenschaften gelegt durch die rückwirkend auch wieder Schlüsse auf den Depositionsmechanismus gezogen werden konnten.

Laserdeponierte PMMA-Filme zeigen einen glatten Filmanteil mit Oberflächenrauigkeiten im Bereich unter einem nm, der vermutlich durch Reaktion von Bruchstücken des Target-Materials entstanden ist, die hauptsächlich kleiner als die Monomereinheit sind. Die zweite Komponente besteht aus Tröpfchen aus PMMA (Droplets), deren Kettenlänge durch die Ablation stark verkürzt ist und deren molekulare Masse bei $M_{W}=8,0 \mathrm{~kg} / \mathrm{mol}$ liegt.

Für den Ablationsmechanismus von PMMA bei der verwendeten Wellenlänge von $248 \mathrm{~nm}$ konnte ein zweistufiger Prozess bestimmt werden. Reines PMMA hat eine so große mittlere Eindringtiefe ( $150 \mu \mathrm{m})$, dass die Energie pro Volumen nicht ausreicht, um Material abzutragen. Dies ändert sich in einem ersten Schritt, der so genannten Inkubation. Hier wird das Polymer durch einen photochemischen Mechanismus verändert. Diese Veränderungen betreffen vor allem die Esterseitengruppe des PMMA, da diese die UV-Strahlung absorbiert. Die Veränderungen bewirken Kettenlängenverkürzung und die Bildung von zusätzlichen Doppelbindungen und damit weiterer Chromophoren, was lokal die Absorption erhöht. Auf diese Weise kann dann durch Energieabsorption in diesen Bereichen die Temperatur stark genug ansteigen, um das Polymer photothermisch abzutragen. Da dieser lokale Prozess statistisch verteilt unter der Oberfläche stattfindet (sub surface heating), bilden sich Blasen mit flüssigem und gasförmigem Polymer. Wenn diese platzen, werfen sie strahlförmig unter Druck flüssiges PMMA mit durch die Inkubation auf $8,0 \mathrm{~kg} / \mathrm{mol}$ reduzierte Molmasse aus, und dieses schlägt sich auf dem Film in Form von Droplets mit typischerweise 1-4 $\mu \mathrm{m}$ Durchmesser nieder. Es konnte gezeigt werden, dass die Moleküle mit diesen Molmassen 
übertragen werden und nicht, wie in der Vergangenheit vermutet, durch Repolymerisation auf dem Substrat entstehen. Bei den verwendeten Laserenergien von 1,2 J/ $\mathrm{cm}^{2}$ wird schon ab den ersten Pulsen Material abgetragen, ein Gleichgewichtszustand zwischen Inkubation und Abtrag wird aber erst nach ca. 70 Pulsen erreicht.

Durch Erhöhung der Substrattemperatur auf über $125^{\circ} \mathrm{C}$ während der Deposition lässt sich die Molmasse des Filmmaterials weiter erhöhen. Sie steigt auf über $3,2 \cdot 10^{4} \mathrm{~g} / \mathrm{mol}$ bei $250{ }^{\circ} \mathrm{C}$ wobei hier gezeigt werden konnte, dass es sich nicht um linear aneinander gewachsene verkürzte Polymerketten handeln kann.

Nach dieser Klärung des Depositionsmechanismus scheint die einzige Möglichkeit, Filme zu erhalten, die chemisch näher am Ausgangsmaterial sind und die weniger von den Droplets dominiert werden, den Einfluss der Inkubationsphase zu verringern. Um starke Absorption und damit Ablation auch ohne Zerstörung des Materials und zusätzlich nahe der Oberfläche zu ermöglichen, wurde dieses so modifiziert, dass es schon im Ausgangszustand eine deutlich geringere Eindringtiefe für UV-Strahlung hat. Der erste Schritt hierzu, die Beimengung von bis zu 10 Gew.\% Antracen, zeigt, dass dies Veränderungen in die gewünschte Richtung zur Folge hat. Die Ablation am Target konnte stabilisiert werden, die Droplets auf den Filmen wurden weniger dominant und die Kettenlänge des Polymers konnte auf 14,3 kg/mol erhöht werden. Jedoch scheint die Verteilung des Antracens mikroskopisch nicht homogen genug zu sein, als dass die Instabilität vollständig unterdrückt werden könnte.

Als Konsequenz darauf wurde ein Polymer synthetisiert, für das mittels RAFT-Polymerisation an jedes Makromolekül ein Absorber angehängt war. Auch hier konnte gezeigt werden, dass die Inkubationsphase verkürzt war, jedoch war durch die geringe Absorberkonzentration die Stabilität des Targets unzureichend.

Ein weiterer Versuch, statt eines einzelnen Absorbers eine ganze Kette hochabsorbierenden Polystyrols zu verwenden, konnte diese Stabilität deutlich verbessern und die DropletAnzahl deutlich reduzieren, nur die Kettenlängen des Polymers sind immer noch deutlich kleiner als beim Ausgangsmaterial.

Als konsequente Maximierung der Targetmodifikationen wurde eine Matrix Assisted Pulsed Laser Evaporation (MAPLE) Apparatur neu aufgebaut. Hier ist der Absorber in Form eines stark UV-Licht absorbierenden Lösungsmittels gegenüber dem erwünschten Filmmaterial in der Mehrheit. Die Polymerlösung wird in flüssigem Stickstoff schockgefroren und kann dann als Target verwendet werden. Experimente mit zwei verschiedenen Lösungsmitteln und einem zusätzlichen inerten Hintergrundgas zeigen, dass der Mecha- 
nismus grundlegend geändert werden konnte. Vor allem Toluol als Lösungsmittel scheint geeignet zu sein, die Anforderungen gut zu erfüllen. Die chemische Struktur ist deutlich näher am Ausgangsmaterial und es ergeben sich vielversprechende Möglichkeiten auch im Hinblick auf andere organische Materialien.

Zur Untersuchung der mechanischen Eigenschaften der laserdeponierten Filme wurde mechanische Spektroskopie verwendet, um die temperatur- und frequenzabhängigen Moduln zu untersuchen. Als in-situ Untersuchungsmethode wurde hierfür das Plasma Puls angeregte Reed (Plasma Plume eXcited Reed, PPXR) neu entwickelt. Hierbei handelt es sich um einen mit geringer Dämpfung frei schwingenden Balken, entsprechend einem konventionellen vibrating reed. Auf diesen wird der zu vermessende Film aufgebracht. Die Anregung der Schwingung des Balkens erfolgt für das PPXR über die Energie des Laserplasmas, das von einem metallischen Silbertarget ausgeht. Auf diese Weise lässt sich in-situ mit hoher Auflösung der komplexe E-Modul des Films bestimmen.

Die Messungen mit PPXR zeigen, dass vor allem der Imaginärteil des komplexen elastischen Moduls, der Verlustmodul, sehr gut bestimmt werden kann. Der temperaturabhängige Verlauf folgt den Erwartungen und die Anpassung einer Havriliak-Negami-Funktion zeigt Parameter, die mit den bekannten Veränderungen in laserdeponiertem PMMA konsistent sind.

Durch die in-situ Messung während der Auslagerung des laserdeponierten PMMA konnte bis zu einer Temperatur von $250{ }^{\circ} \mathrm{C}$ die Relaxation des Materials beobachtet werden, bei höheren Temperaturen wird es dann weitgehend zersetzt.

Der Vergleich mit anderen Spektroskopiemethoden, speziell dem Doppelpaddeloszillator (DPO) und der dielektrischen Spektroskopie zeigt den Wert des PPXR. Im Vergleich zum DPO zeigt sich für die hier durchgeführten Messungen eine vergleichbare Präzision, da jedoch hauptsächlich der elastische Modul, und nicht wie beim DPO der Schermodul bestimmt wird, ergeben sich komplementäre Informationen. Im Vergleich zur dielektrischen Spektroskopie konnte gezeigt werden, dass die mechanische Spektroskopie hauptsächlich die mit dem Glasübergang verbundene $\alpha$-Relaxation misst, während bei der dielektrischen die $\beta$-Relaxation, eine Rotation der Estergruppe, dominiert.

Auch die Abhängigkeit der Messung von der Resonanzfrequenz konnte untersucht werden. Auch wenn die Präparation der geeigneten Substrate aufwendig ist und die Qualität der Messung deshalb schwankt, konnte gezeigt werden, dass PPXR sehr gut im Frequenzbereich von 500-7500 Hz eingesetzt werden kann. Durch diese Änderungen verschiebt sich erwartungsgemäß hauptsächlich das Maximum der $\alpha$-Relaxation auf der Temperaturskala. 
Durch die Untersuchung der Filme, die bei unterschiedlichen Substrattemperaturen hergestellt wurden, konnte gezeigt werden, dass die Änderung der Kettenlänge zu gering ist, um eine Veränderung der Glasübergangstemperatur zu beobachten. Allerdings konnten bei sehr hohen Substrattemperaturen von $225^{\circ} \mathrm{C}$ Rückschlüsse auf den Mechanismus des Kettenwachstums während der Deposition gewonnen werden. Die Ergebnisse lassen darauf schließen, dass das Wachstum nicht über das lineare Anwachsen von Ketten erfolgt und auch nicht über die Repolymerisation von Monomeren, wie dies in der Vergangenheit häufig angenommen wurde. Stattdessen wird vorgeschlagen, dass es sich um ein Quervernetzen der vorhandenen Makromoleküle handelt, die bei hohem Vernetzungsgrad bei hohen Temperaturen nicht mehr flüssig werden und in starkem Maße duroplastischen Charakter zeigen.

Insgesamt konnte im Verlauf dieser Arbeit das Bild der gepulsten Laserdeposition von PMMA bedeutend erweitert werden. Der Ablations- und Depositionsmechanismus wurde besser verstanden und es konnte als Konsequenz aus diesem Verständnis gezeigt werden, dass eine gezielte Veränderung der Targets Änderungen der Eigenschaften in die gewünschte Richtung ermöglicht. Durch die neue Methode, PPXR, ermöglichte die mechanische Spektroskopie an laserdeponierten Filmen neue Erkenntnisse, unter anderem über den Mechanismus des Kettenwachstums bei erhöhten Substrattemperaturen.

Aus diesen neuen Erkenntnissen lassen sich Schlussfolgerungen ableiten, welche Eigenschaften ein makromolekulares Material haben muss, damit erfolgreich mittels der gepulsten Laserdeposition Filme deponiert werden können. PMMA kann also in diesem Sinn als Modellsystem für die Laserdeposition von organischen Materialien angesehen werden. 


\section{Literaturverzeichnis}

[1] Alvarez, F. ; Alegría, A. ; Colmenero, J. : Relationship between the timedomain Kohlrausch-Williams-Watts and frequency-domain Havriliak-Negami relaxation functions. In: Phys. Rev. B 44 (1991) 7306

[2] Alvarez, F. ; Alegría, A. ; Colmenero, J. : Interconnection between frequencydomain Havriliak-Negami and time-domain Kohlrausch-Williams-Watts relaxation functions. In: Phys. Rev. B 47 (1993) 125

[3] Andrew, J. E.; Dyer, P. E.; Forster, D. ; Key, P. H.: Direct etching of polymeric materials using a XeCl laser. In: Appl. Phys. Lett. 43 (1983) 717

[4] Angell, C. A.: Structural instability and relaxation in liquid and glassy phases near the fragile liquid limit. In: J. Non-Cryst. Solids 102 (1988) 205

[5] Bengtzelius, U. ; Götze, W. ; Sjölander, A. : Dynamics of supercooled liquids and the glass transition. In: J. Phys. C: Solid State Phys. 17 (1984) 5915

[6] Bergman, R. ; Alvarez, F. ; Alegría, A. ; Colmenero, J. : The merging of the dielectric $\alpha$ - and $\beta$-relaxations in poly-(methyl methacrylate). In: J. Chem. Phys. 109 (1998) 7546

[7] Blanchet, G. B.; Fincher, Jr., C. R.: Laser induced unzipping: A thermal route to polymer ablation. In: Appl. Phys. Lett. 65 (1994) 1311

[8] Blanchet, G. B.; Fincher, Jr., C. R.; Jackson, C. L.; Shah, S. I. ; Gardner, K. H.: Laser Ablation and the Production of Polymer Films. In: Science 262 (1993) 719

[9] Blanchet, G. B.: Deposition of Poly(methyl methacrylate) Films by UV Laser Ablation. In: Macromolecules 28 (1995) 4603

[10] Blanchet, G. B.; Cotts, P. ; Fincher, JR, C. R.: Incubation: Subthreshold ablation of poly-(methyl methacrylate) and the nature of the decomposition pathways. In: J. Appl. Phys. 88 (2000), Nr. 52975

[11] Brandenburg, J. ; Neu, V. ; Holzapfel, B. ; Krebs, H.-U. ; Fähler, S. : Pulsed laser deposition of metals: Consequences of the energy distribution within the laser spot. In: Appl. Phys. A 79 (2004) 1005

[12] Buback, M. ; Hesse, P. ; Junkers, T. ; Vana, P. : Determination of Addition and Fragmentation Rate Coefficients in RAFT Polymerization via Time-Resolved ESR Spectroscopy after Laser Pulse Initiation. In: Macromol. Rapid Commun. 27 (2006) 182 
[13] Bubb, D. M.; Horwitz, J. S.; Callahan, J. H.; McGill, R. A.; Houser, E. J.; Chrisey, D. B.; Papantonakis, M. R.; Haglund, Jr., R. F.; Galicia, M. ; VERTES, A. : Resonant infrared pulsed-laser deposition of polymer films using a free-electron laser. In: J. Vac. Sci. Technol. A 19 (2001) 2698

[14] Bubb, D. M.; Papantonakis, M. R.; Toftmann, B. ; Horwitz, J. S.; McGill, R. A.; Chrisey, D. B. ; Haglund, JR., R. F.: Effect of ablation parameters on infrared pulsed laser deposition of poly(etylene glycol) films. In: J. Appl. Phys. 91 (2002) 9809

[15] Bubb, D. M.; Ringeisen, B. R.; Callahan, J. H.; Galicia, M. ; Vertes, A. ; Horwitz, J. S.; McGill, R. A.; Houser, E. J.; Wu, P. K.; Piqué, A. ; Chrisey, D. B.: Vapor deposition of intact polyethylene glycol thin films. In: Appl. Phys. A 73 (2001) 121

[16] Bubb, D. M.; Wu, P. K.; Horwitz, J. S.; Callahan, J. H.; Galicia, M. ; Vertes, A. ; McGill, R. A.; Houser, E. J.; Ringeisen, B. R. ; Chrisey, D. B.: The effect of the matrix on film properties in matrix-assisted pulsed laser evaporation. In: $J$. Appl. Phys. 91 (2002) 2055

[17] Buchdahl, R. ; Morgan, R. J. ; Nielsen, L. E.: Free Vibrating Reed Apparatus for the Measurement of Internal Friction and Young's Molulus down to 4K. In: Rev. Sci. Instrum. 41 (1970) 1342

[18] Chrisey, D. B. (Hrsg.); Hubler, G. K. (Hrsg.): Pulsed Laser Deposition Of Thin Films. New York : Wiley Interscience Publication, 1994. - ISBN 0-471-59218-8

[19] Chrisey, D. B.; Piqué, A. ; McGill, R. A.; Horwitz, J. S.; Ringeisen, B. R.; BubB, D. M. ; Wu, P. K.: Laser Deposition of Polymer and Biomaterial Films. In: Chem. Rev. 103 (2003) 553

[20] Cole, S. ; Cole, R. H.: Dispersion and Absorption in Dielectrics. In: J. Chem. Phys. 9 (1941) 341

[21] Davidson, D. W.; Cole, R. H.: Dielectric Relaxation in Glycerine. In: J. Chem. Phys. 18 (1950) 1417

[22] Davidson, D. W.; Cole, R. H.: Dielectric Relaxation in Glycerol, Propylene Glycol, and $n$-Propanol. In: J. Chem. Phys. 19 (1951) 1484

[23] Debenedetti, P. G.; Stillinger, F. H.: Supercooled liquids and the glass transition. In: Nature 410 (2001) 259

[24] DeBiJe, P. : Zur Theorie der anormalen Dispersion im Gebiete der langwelligen elektrischen Strahlung. In: Ber. D. Phys. Ges. 16 (1913) 777 
[25] DeGennes, P. G.: Reptation of a Polymer Chain in the Presence of Fixed Obstacles. In: J. Chem. Phys. 55 (1971) 572

[26] Dhont, J. K. G. (Hrsg.); Gompper, G. (Hrsg.) ; Richter, D. (Hrsg.): Soft Matter - Complex Materials on Mesoscopic Scales, 33th IFF winter school. Jülich : Forschungszentrum Jülich, 2002 (Materie und Material). - ISBN 3-89336-297-5

[27] Dyer, P. E.: Excimer laser polymer ablation: twenty years on. In: Appl. Phys. A 77 (2003) 167

[28] Dyer, P. E.; Srinivasan, R. : Nanosecond photoacoustic studies on ultraviolet laser ablation of organic polymers. In: Appl. Phys. Lett. 48 (1986) 445

[29] Eason, R. (Hrsg.): Pulsed Laser Deposition Of Thin Films: applications-led growth of functional materials. Hoboken, New Jersey : Wiley Interscience Publication, 2007. - ISBN 0-471-44709-9

[30] Ediger, M. D.; Angell, C. A. ; Nagel, S. R.: Supercooled Liquids and Glasses. In: J. Phys. Chem. 100 (1996) 13200

[31] Efthimiopoulos, T. ; Kiagias, C. ; Heliotis, G. ; Helidonis, E. : Evidence of volume bubble creation during laser ablation of PMMA organic polymer. In: Can J. Phys. 78 (2000) 509

[32] Estler, R. C.; Nogar, N. S.: Mass spectroscopic identification of wavelength dependent UV laser photoablation fragments from polymethylmethacrylate. In: Appl. Phys. Lett. 49 (1986) 1175

[33] F̈̈hler, S. ; Störmer, M. ; Krebs, H.-U. : Origin and avoidance of droplets during laser ablation of metals. In: Appl. Surf. Sci. 109-110 (1997) 433

[34] FäHLER, S. ; KREBS, H.-U. : Calculations and experiments of material removal and kinetic energy during pulsed laser ablation of metals. In: Appl. Surf. Sci. 96-98 (1996) 61

[35] FAUPEL, J. : Wachstum von metallischen Nanoclustern auf Polymeroberflächen, Universität Göttingen, Diss., 2005. http://webdoc.sub.gwdg.de/diss/2005/faupel/ faupel.pdf. - Elektronische Ressource

[36] Faupel, J. ; Fuhse, C. ; Meschede, A. ; Herweg, C. ; Krebs, H.-U. ; VitTA, S. : Microstructure of pulsed laser deposited ceramic-metal and polymer-metal nanocomposited thin films. In: Appl. Phys. A 79 (2004) 1233

[37] Fitz-Gerald, J. M.; Jennings, G. ; Johnson, R. ; Fraser, C. L.: Matrix assisted pulsed laser deposition of light emmitting polymer thin films. In: Appl. Phys. A 80 (2005) 1109 
[38] Fliessbach, T. : Lehrbuch zur theoretischen Physik. Heidelberg, Berlin, Oxford : Spektrum Akademischer Verlag, 1996. - ISBN 3-86025-686-6

[39] Fuhse, C. ; Krebs, H.-U. ; Vitta, S. ; Johansson, G. A.: Interface quality and thermal stability of laser deposited metal/MgO multilayers. In: Appl. Opt. 43 (2004) 6265

[40] Fukumura, H. ; Mibuka, N. ; Shigeru, E. ; Masuhara, H. ; Nishi, N. : Mass Spectrometric Studies on Laser Ablation of Polystyrene Sensitized with Anthracene. In: J. Phys. Chem. 97 (1993) 13761

[41] Fulcher, G. S.: Analysis of Recent Measurements of the Viscosity of Glasses. In: J. Am. Ceram. Soc. 8 (1925) 339

[42] Garwe, F. ; Schönhals, A. ; Lockwenz, H. ; Beiner, M. ; Schröter, K. ; Donth, E. : Influence of Cooperative $\alpha$ Dynamics on Local $\beta$ Relaxation during the Development of the Dynamic Glass Transition in Poly(n-alkyl methacrylate)s. In: Macromolecules 29 (1996) 247

[43] Gavigan, J. P.; Givord, D. ; Lienard, A. ; Rebouillat, J. P. ; Souche, Y. : Epitaxial growth and magnetic properties of $\mathrm{RM}_{5}$ thin films ( $\mathrm{R}=$ rare earth, $\mathrm{M}=\mathrm{Ni}, \mathrm{Co}, \mathrm{Fe})$ prepared by laser ablation deposition. In: J. Appl. Phys. 69 (1991) 5677

[44] Ghosh, A. P.; Hurst, JR., J. E.: Photoacoustic studies of excimer laser induced ablation of polymethylmethacrylate. In: J. Appl. Phys. 64 (1988) 287

[45] Hansen, S. G.; Robitaille: Arrival time measurements of films formed by pulsed laser evaporation of polycarbonate and selenium. In: J. Appl. Phys. 64 (1988) 2122

[46] Hansen, S. G.; Robitaille: Formation of polymer films by pulsed laser evaporation. In: Appl. Phys. Lett. 52 (1988) 81

[47] Harms, U. ; Kempen, L. ; Neuhäuser, H. : Influence of stress in thin film modulus measurements by the vibrating reed technique. In: Thin Solid Films 153-157 (1998) 323

[48] Harms, U. ; NeuhäUser, H. : Vibrating reed apparatus with optical detection and digital signal processing: Application to measurements on thin films. In: Rev. Sci. Instrum. 70 (1999) 1751

[49] Havriliak, S. ; Negami, S. : A Complex Plane Representation of Dielectric and Mechanical Relaxation Process in Some Polymers. In: Polymer 8 (1967) 161

[50] Hellwege, K.-H. (Hrsg.): Landolt-Börnstein, Numerical Data and Functional Relationships in Science and Technology. Bd. III-18. Berlin, Heidelberg, New York, Tokyo : Springer Verlag, 1984. - ISBN 3-86025-686-6 
[51] Hiraoka, H. ; Chuang, T. J. ; Masuhara, H. : Dopant-induced ablation of polymers by $308 \mathrm{~nm}$ excimer laser. In: J. Vac. Sci. Technol. B. 6 (1988) 463

[52] HöLCK, O. : Aufbau einer Vibrating-Reed-Apparatur zur Untersuchung der inneren Reibung kleiner Moleküle in polymeren Gläsern. Institut für Materialphysik, Universität Göttingen, Diplomarbeit, 2000

[53] Hölzle, R. (Hrsg.): Physik der Polymere, 22. IFF-Ferienkurs. Jülich : Forschungszentrum Jülich, 2002. - ISBN 3-89336-055-7

[54] Inagaki, N. : Plasma Surface Modification and Plasma Polymerization. Lancester, Pennsylvania : Technomic Publishing Company, Inc., 1996. - ISBN 1-56676-337-1

[55] JellineK, H. H. G.; LuH, M. D.: Thermal Degradation of Polymethylmethacrylate. In: Makromol. Chem. 115 (1968) 89

[56] Jones, R. M.: Mechanics Of Composite Materials. Philadelphia : Taylor and Francis, 1999. - ISBN 1-56032-712-X

[57] Kohlrausch, R. : Theorie der elektrischen Rückstandes in der Leidener Flasche. In: Ann. Phys. 91 (1854) 56-82, 179-214

[58] Koppelmann, J. ; Hirnböck, R. ; Leder, H. ; Royer, F. : Relaxation spectroscopy on amorphous high polymers. In: Colloid 6 Polymer Sci. 258 (1980) 9

[59] Kramers, H. A.: Die Dispersion und Absorption von Röntgenstrahlen. In: Phys. Z. $30(1929) 522$

[60] KREBS, H.-U. : Characteristic properties of laser deposited metallic systems. In: Intern. J. Non-Equilibrium Processing 10 (1997) 3

[61] Krebs, H.-U. ; F̈̈HLer, S. ; Bremert, O. : Laser deposition of metallic alloys and multilayers. In: Appl. Surf. Sci. 86 (1995) 86

[62] Krebs, H.-U. ; Weisheit, M. ; Faupel, J. ; Süske, E. ; Scharf, T. ; Fuhse, C. ; Störmer, M. ; Sturm, K. ; Seibt, M. ; KiJewski, H. ; Nelke, D. ; Panschenko, E. ; Buback, M. : Pulsed laser deposition (PLD) - a versatile thin film technique. In: Adv. in Solid State Phys. 43 (2003) 505

[63] Kronig, R. d. L.: On the theory of dispersion of x'rays. In: J. Opt. Soc. Am. 12 (1926) 547

[64] Kuebler, S. C.; Schaefer, D. J.; Bofffel, C. ; Pawelzik, U. ; Spiess, H. W.: 2D Exchange NMR Investigation of the $\alpha$-Relaxation in Poly(ethyl methacrylate) as Compared to Poly(methyl methacrylate). In: Macromolecules 30 (1997) 6597 
[65] Küper, S. ; Modaressi, S. ; Stuke, M. : Photofragmentation Pathways of a PMMA Model Compound under UV Excimer Laser Ablation Conditions. In: J. Phys. Chem. 94 (1990) 7514

[66] Küper, S. ; Stuke, M. : Femtosecond uv Excimer Laser Ablation. In: Appl. Phys. $B 44$ (1987) 199

[67] Küper, S. ; Stuke, M. : UV-Excimer-Laser Ablation of Polymethylmethacrylate at $248 \mathrm{~nm}$ : Characterization of Incubation Sites with Fourier Transform IR- and UV-Spectroscopy. In: Appl. Phys. A 49 (1989) 211

[68] KurŠumović, A. ; CAntor, B. : Anelastic crossover and creep recovery spectra in $\mathrm{Fe}_{40} \mathrm{Ni}_{40} \mathrm{~B}_{20}$ metallic glass. In: Scripta Materialia 34 (1996) 1655

[69] Lakes, R. S.: High Damping Composite Materials: Effect of Structural Hierarchy. In: J. Composite Materials 36 (2002) 287

[70] Lazare, S. ; Lopez, J. ; Weisbuch, F. : High-aspect-ratio microdrilling in polymeric materials with intense KrF laser radiation. In: Appl. Phys. A 69 [Suppl.] (1999) $\mathrm{S} 1$

[71] Leutheusser, E. : Dynamic model of the liquid-glass transition. In: Phys. Rev. A 29 (1984) 2765

[72] Lippert, T. ; Webb, R. L.; Langford, S. C. ; Dickinson, J. T.: Dopand induced ablation of poly(methyl methacrylate) at 308 nm. In: J. Appl. Phys. 85 (1999), Nr. 31838

[73] Lippert, T. : Interaction of Photons with Polymers: From Surface Modification to Ablation. In: Plasma Process. Polym. 2 (2005) 525

[74] LÖSEKRUG, B. : Energiedichteabhängigkeit der Eigenschaften laserdeponierter Poly(methyl methacrylat)-Schichten und-Kompositmaterialien. Institut für Materialphysik, Universität Göttingen, Diplomarbeit, 2006

[75] Lu, H. ; Zhang, X. ; Kauss, W. : Uniaxial, Shear, and Poisson Relaxation and Their Conversion to Bulk Relaxation: Studies on Poly(Methyl Methacrylate). In: Polymer Engineering and Science 37 (1997) 1053

[76] Manring, L. E.: Thermal Degradation of Poly(methyl methacrylate). 4. Random Side-Group Scission. In: Macromolecules 24 (1991) 3304

[77] Mercado, A. L.; Allmond, C. E.; Hoekstra, J. G. ; Fitz-Gerald, J. M.: Pulsed laser deposition vs. matrix assisted pulsed laser evaporation for growth of biodegradable polymer thin films. In: Appl. Phys. A 81 (2005) 591 
[78] Meschede, A. : Wachstum und Eigenschaften laserdeponierter $\mathrm{MgO} / \mathrm{Fe}$ - und Ti$\mathrm{Sc} / \mathrm{Fe}$-Cr-Schichtsysteme. Institut für Materialphysik, Universität Göttingen, Diplomarbeit, 2004

[79] Miotello, A. ; Kelly, R. : Critical assessment of thermal models for laser sputtering at high fluences. In: Appl. Phys. Lett. 67 (1995) 3535

[80] Moad, G. ; Rizzardo, E. ; Thang, S. H.: Living Radical Polymerization by the RAFT Process. In: Aust. J. Chem. 58 (2005) 379

[81] Mortimer, C. E.; Müller, U. : Chemie. Stuttgart : Georg Thieme Verlag, 2003. - ISBN 3-134-84308-0

[82] Muzeau, E. ; Perez, J. ; Johari, G. P.: Mechanical Spectrometry of the $\beta$ Relaxation in Poly(methyl methacrylate). In: Macromolecules 24 (1991) 4713

[83] O’Driscoll, K. ; Amin Sanayei, R. : Chain-Length Dependence of the Glass Transition Temperature. In: Macromolecules 24 (1991) 4479

[84] Parkus, H. : Mechanik der festen Körper. Wien-New York : Springer-Verlag, 1995. - ISBN 3-211-80777-2

[85] Parrat, L. G.: Surface Studies of Solids by Total Reflection of X-Rays. In: Phys. Rev. 95 (1954) 359

[86] Pettit, G. H.; Sauerbrey, R. : Pulsed Ultraviolet Laser Ablation. In: Appl. Phys. A 56 (1993) 51

[87] Piqué, A. ; McGill, R. A.; Chrisey, D. B.; Leonhardt, D. ; Mslna, T. E.; Spargo, B. J.; Callahan, J. H.; Vachet, R. W.; Chung, R. ; Bucaro, M. A.: Growth of organic thin films by the matrix assisted pulsed laser evaporation (MAPLE) technique. In: Thin Solid Films 355-356 (1999) 536

[88] RabeK, J. F.: Photodegradation of Polymers. Berlin; Heidelberg; New York : Springer-Verlag, 1996. - ISBN 3-540-60716-1

[89] Rebollar, E. ; Gaspard, S. ; M., O. ; Villavieja, M. M.; Corrales, T. ; Bosch, P. ; Georgiou, S. ; Castillejo, M. : Pulsed laser deposition of polymers doped with fluorescent molecular sensors. In: Appl. Phys. A 84 (2006) 171

[90] Ringeisen, B. R.; Callahan, J. ; Wu, P. K.; Piqué, A. ; Spargo, B. ; McGill, R. A.; Bucaro, M. ; Kim, H. ; Bubb, D. M. ; Chrisey, D. B.: Novel Laser-Based Deposition of Active Protein Thin Films. In: Langmuir 17 (2001) 3472

[91] RöDER, J. ; KREBS, H.-U. : Tuning the microstructure of pulsed laser deposited polymer-metal nanocomposites. In: Appl. Phys. A 85 (2006) 15 
[92] RöDER, J. : Beeinflussung des Wachstums von Ag auf PMMA. Institut für Materialphysik, Universität Göttingen, Diplomarbeit, 2005

[93] Rodrigo, K. ; Czuba, P. ; Toftmann, B. ; Schou, J. ; Pedrys, R. : Surface morphology of polyetylene glycol films produced by matrix-assisted pulsed laser evaporation (MAPLE): Dependence on substrate temperature. In: Appl. Surf. Sci 252 (2006) 4824

[94] Rodrigo, K. ; Toftmann, B. ; Schou, J. ; Pedrys, R. : Laser-induced ion emision during polymer deposition from a flash-frozen water ice matrix. In: Chem. Phys. Lett. 399 (2004) 368

[95] Rösner, P. ; Samwer, K. ; Pohl, R. O. ; Schneider, S. : Use of a double-paddle oscillator for the study of metallic films at high temperatures. In: Rev. Sci. Instrum. 74 (2003) 3395

[96] Rösner, P. ; Hachenberg, J. ; Samwer, K. ; Wehn, R. ; Lunkenheimer, P. ; Loidl, A. ; Süske, E. ; Scharf, T. ; Krebs, H.-U. : Comparison of mechanical and dielectric relaxation processes in laser deposited poly(methyl methacrylate) films. In: New J. Phys. 8 (2006) 89

[97] Rösner, P. C.: Mechanische Relaxation in komplexen Fluiden, Universität Göttingen, Diss., 2004. http://webdoc.sub.gwdg.de/diss/2004/roesner/roesner.pdf.

- Elektronische Ressource

[98] ScharF, T. : Mechanische Spannungen und Struktur laserdeponierter metallischer Filme in Abhängigkeit von der Teilchenenergie. Institut für Materialphysik, Universität Göttingen, Diplomarbeit, 2001

[99] Scharf, T. ; Faupel, J. ; Sturm, K. ; Krebs, H.-U. : Intrinsic stress evolution in laser deposited thin films. In: J. Appl. Phys. 94 (2003) 4273-4278

[100] Scharf, T. ; Faupel, J. ; Sturm, K. ; Krebs, H.-U. : Pulsed laser deposition of metals in various inert gas atmospheres. In: Appl. Phys. A 79 (2004) 1587-1598

[101] Scharf, T. ; Krebs, H.-U. : Influence of an inert gas pressure on the deposition rate during pulsed laser deposition. In: Appl. Phys. A 75 (2002) 551-554

[102] Scharf, T. ; KreBs, H.-U. : In-situ mechanical spectroscopy of laser deposited films using plasma plume excited reed. In: Rev. Sci. Instrum. 77 (2006) 093901

[103] Schmidt, H. ; Ihlemann, J. ; Wolff-Rottke, B. ; Luther, K. ; Troe, J. : Ultraviolet laser ablation of polymers: spot size, pulse duration, and plume attenuation effects explained. In: J. Appl. Phys. 83 (1998) 5458 
[104] Schmidt-Rohr, K. ; Kulik, A. S.; Beckham, H. W.; Ohlemacher, A. ; PawelZIK, U. ; Boeffel, C. ; SpIEss, H. W.: Molecular Nature of the $\beta$ Relaxation in Poly(methyl metharcylate) Investigated by Multidimensional NMR. In: Macromolecules 27 (1994) 4733

[105] Schröter, K. ; Unger, R. ; Reissig, S. ; Garwe, F. ; Kahle, S. ; Beiner, M. ; Donth, E. : Dielectric Spectroscopy in the $\alpha \beta$ Splitting Region of Glass Transition in Poly(ethyl methacrylate) and Poly(n-butyl methacrylate): Different Evaluation Methods and Experimental Conditions. In: Macromolecules 31 (1998) 8966

[106] Sellinger, A. T.; Leveugle, E. M.; Gogick, K. ; Zhigilei, L. V. ; Fitz-Gerald, J. M.: Laser processing of polymer nanocomposite thin films. In: J. Vac. Sci. Technol. A 24 (2006) 1618

[107] Singer, K. D.; King, L. A.: Relaxation phenomena in polymer nonlinear optical materials. In: J. Appl. Phys. 70 (1991) 3251

[108] SRinivasan, R. : Ablation of polymethyl methacrylate films by pulsed (ns) ultraviolet and infrared $(9.17 \mu \mathrm{m})$ lasers: A comparative study by ultrafast imaging. In: J. Appl. Phys. 73 (1993) 2743

[109] Srinivasan, R. ; Braren, B. ; Casey, K. G.: Nature of "incubation pulses" in the ultraviolet laser ablation of polymethyl methacrylate. In: J. Appl. Phys. 68 (1990) 1842

[110] Srinivasan, R. ; Braren, B. ; Casey, K. G. ; YeH, M. : Ultrafast imaging of ultraviolet laser ablation and etching of polymethylmetharcylate. In: Appl. Phys. Lett. 55 (1989) 2790

[111] Srinivasan, R. ; Braren, B. ; Seeger, D. E. ; Dreyfus, R. W.: Photochemical Cleavage of a Polymeric Solid: Details of the Ultraviolet Laser Ablation of Poly(methyl methacrylate) at 193 and 248 nm. In: Macromolecules 19 (1986) 916

[112] Srinivasan, R. ; Mayne-Banton, V. : Self-developing photoetching of poly(ethylene terephthalate) films by far-ultraviolet excimer laser radiation. In: Appl. Phys. Lett. 41 (1982) 576

[113] Srinivasan, R. ; Smrtic, M. A. ; Babu, S. V.: Excimer laser etching of polymers. In: J. Appl. Phys. 59 (1986) 3861

[114] Stillinger, F. H.: A Topographic View of Supercooled Liquids and Glass Formers. In: Science 267 (1995) 1935

[115] Sturm, K. : Einfluss einer Argon-Atmosphäre auf die gepulste Laserdeposition von Metallschichten, Universität Göttingen, Diss., 2000. - Cuvillier Verlag Göttingen, ISBN 3-89712-909-4 
[116] Süske, E. : Laserdeposition von Polymer-Metall-Schichten. Institut für Materialphysik, Universität Göttingen, Diplomarbeit, 2001

[117] Süske, E. : Charakteristische Eigenschaften laserdeponierter Poly(methyl-methacrylat)-Filme, Universität Göttingen, Diss., 2005. http://webdoc.sub.gwdg.de/ diss/2005/sueske/sueske.pdf. - Elektronische Ressource

[118] Süske, E. ; Scharf, T. ; Krebs, H.-U. ; Junkers, T. ; Buback, M. : Mechanism of poly(methyl methacrylate) film formation by pulsed laser deposition. In: J. Appl. Phys. 100 (2006) 014906

[119] Süske, E. ; Scharf, T. ; Krebs, H.-U. ; Panchenko, E. ; Junkers, T. ; Egorov, M. ; Buback, M. ; KiJewski, H. : Tuning of cross-linking and mechanical properties of laser-deposited poly (methyl metacrylate) films. In: J. Appl. Phys. 97 (2005) 063501-1-063501-4

[120] Süske, E. ; Scharf, T. ; Schaaf, P. ; Panchenko, E. ; Nelke, D. ; Buback, M. ; KIJEWsKi, H. ; KreBs, H.-U. : Variation of the mechanical properties of pulsed laser deposited PMMA films during annealing. In: Appl. Phys. A 79 (2004) 1295-1297

[121] Sutcliffe, E. ; Srinivasan, R. : Dynamics of UV laser ablation of organic polymer surfaces. In: J. Appl. Phys. 60 (1986) 3315

[122] Tammann, G. ; Hesse, W. : Die Abhängigkeit der Viscosität von der Temperatur bei unterkühlten Flüssigkeiten. In: Z. Anorg. Allg. Chem. 156 (1926) 245

[123] Timm, R. ; Willmott, P. R. ; Huber, J. R.: Ablation and blow-off characteristics at $248 \mathrm{~nm}$ of $\mathrm{Al}, \mathrm{Sn}$ and Ti targets used for thin film pulsed laser deposition. In: $J$. Appl. Phys. 80 (1996) 1794

[124] Toftmann, B. ; Papantonakis, B. R.; Auyeung, R. C. Y.; Kim, W. ; O’Malley, S. M.; Bubb, D. M.; Horwitz, J. S.; Schou, J. ; Johansen, P. M. ; Haglund, R. F.: UV and RIR matrix assisted pulsed laser deposition of organic MEH-PPV films. In: Thin Solid Films 453-454 (2004) 177

[125] Toftmann, B. ; Rodrigo, K. ; Schou, J. ; Pedrys, R. : High laser fluence deposition of organic materials in water ice matices by "MAPLE". In: Appl. Surf. Sci. 247 (2005) 211

[126] Tsuboi, Y. ; Adachi, N. ; Yamamoto, E. ; Itaya, A. : Pulsed Laser Deposition of Poly(tetrafluoroethylene), Poly(methylmethacrylate) and Polycarbonate Utilizing Anthracene-Photosensitized Ablation. In: Jpn. J. Appl. Phys. 41 (2002) 885

[127] Tsuboi, Y. ; Goto, M. ; Itaya, A. : Thin Film Formation of a Protein by Laser Ablation Deposition Technique. In: Chemistry Letters 27 (1998) 521 
[128] Tsuboi, Y. ; Goto, M. ; Itaya, A. : Pulsed laser deposition of silk protein: Effekt of photosenistized-ablation on the secondary structure in thin deposited films. In: $J$. Appl. Phys. 89 (2001) 7917

[129] Tsuboi, Y. ; Itaya, A. : Deposition of a thin polystyrene film by anthracenephotosensitized laser ablation at 351 nm. In: Appl. Phys. Lett. 74 (1999) 3896

[130] Tsunekawa, M. ; Nishio, S. ; SAto, H. : Laser Ablation of polymethylmethacrylate and polystyrene at $308 \mathrm{~nm}$ : Demonstration of thermal and photothermal mechanisms by a time-of-flight mass spectroscopic study. In: J. Appl. Phys. 76 (1994) 5598

[131] Vitta, S. ; Weisheit, M. ; Scharf, T. ; Krebs, H.-U. : Alloy-ceramic oxide multilayer mirror for water-window soft x rays. In: Optics Lett. 26 (2001) 1448-1450

[132] Vogel, H. : Das Temperaturabhängigkeitsgesetz der Viskosität von Flüssigkeiten. In: Phys. Z. 22 (1921) 645

[133] Williams, G. ; Watts, D. C.: Non-Symetrical Dielectric Relaxation Behaviour Arising from a Simple Empirical Decay Function. In: Trans. Faraday Soc. 66 (1970) 80

[134] Williams, J. ; Shohamy, E. ; Reich, S. ; Eisenberg, A. : Methyl-Group Tunneling in Viscoelastic Relaxation: Experimental Verification. In: Phys. Rev. Lett. 35 (1975) 951

[135] Willmott, P. R.: Deposition of complex multielemental thin films. In: Prog. Surface Science 76 (2004) 163

[136] Willmott, P. R.; Huber, J. R.: Pulsed laser vaporization and deposition. In: Rev. Mod. Phys. 72 (2000) 315

[137] WindT, D. L.: IMD. http://cletus.phys.columbia.edu/〜windt/imd/. Version: 2000

[138] Wu, P. K.; Ringeisen, B. R.; Krizman, D. B.; Frondoza, C. G.; Brooks, M. ; Bubb, D. M.; Auyeung, R. C. Y.; Piqué, A. ; Spargo, B. ; McGill, R. A. ; ChriSEY, D. B.: Laser transfer of biomaterials: Matrix-assisted pulsed laser evaporation (MAPLE) and MAPLE Direct Write. In: Rev. Sci. Instrum. 74 (2003) 2546 


\section{Danksagung}

An erster Stelle möchte ich Prof. Uli Krebs danken, der mir die Möglichkeit gegeben hat, in seiner Arbeitsgruppe diese Arbeit durchzuführen. Er hat während der ganzen Zeit für eine hervorragende Arbeitsatmosphäre gesorgt und stand mir immer mit Rat und vielen hilfreichen Diskussionen zur Seite.

Im Besonderen möchte ich weiterhin allen Mitgliedern meiner Arbeitsgruppe danken. Ich danke den „schon lange Ehemaligen“ Kai Sturm, Martin Weisheit und Christian Fuhse, die durch ihre - teilweise auch unkonventionellen - Lösungen für den Laboralltag vieles möglich gemacht haben. Mit meinen langjährigen Mitstreitern Erik Süske, Jörg Faupel und Andreas Meschede habe ich viele Stunden beim Schrauben im Labor und fruchtbaren Diskussionen verbracht, vielen Dank hierfür. Auch der „nächsten Generation“, Johanna Röder, Britta Lösekrug und Tobias Liese danke ich für ein hervorragendes Arbeitsklima und gute Zusammenarbeit. Conni Mewes und Michael Malchow danke ich besonders für die technische Hilfe bei den vielen Problemen des Laboralltags. Frank Barkuski danke ich für hilfreiche Diskussionen und dafür, dass er Wissen aus dem Laserlabor zu uns mitgebracht hat.

Im Weiteren danke ich dem Rest des Instituts für Materialphysik, besonders dem assimilierten Mitglied der Gruppe und dem im zweiten Stock geduldeten, Carsten Herweg und Sebastian Dreyer, für die angenehme Arbeitsatmosphäre, die sie maßgeblich mit geschaffen haben.

Besonders danken möchte ich den Arbeitsgruppen von Prof. M. Buback und Philipp Vana im Institut für physikalische Chemie, die mit der Chromatographie, der RAFTPolymerisation und vielem sonst, wovon Physiker normalerweise keine Ahnung haben, vieles an dieser Arbeit erst möglich gemacht haben. Auch die stete Bereitschaft zu Diskussionen, vor allem im Rahmen des Graduiertenkollegs, waren unersetzlich. Namentlich danken möchte ich hier Dorit Nelke, Elena Müller, Thomas Junkers, Mark Egorov, Duc Hung Nguyen und Björn Steisel.

Für die mechanische Spektroskopie mit dem DPO und viele fruchtbare Diskussionen dazu danke ich Prof. K. Samwer und Peter Rösner aus dem 1. Physikalischen Institut. Durch diesen Kontakt sind auch die dielektrischen Messungen in Augsburg durchgeführt worden, hier danke ich Prof. A. Loidl, Peter Lunkenheimer und Robert Wehn.

Einige hilfreiche Tipps und viele Diskussionen kamen aus der Polymergruppe von Prof. R. Kirchheim, vor allem von Ole Hölck und Tatjana Haramina.

Ich danke den Mitgliedern des Graduiertenkollegs 782 für die vorbildliche Diskussionsbereitschaft bei den vielen Workshops, besonders Prof. M. Suhm, ohne den diese bestimmt nicht entstanden wäre. Die Anregungen zu der Analyse im Fourier-Raum kamen hier von Prof. C. Griesinger und Robert Schneider, herzlichen Dank.

Zu guter Letzt möchte ich meinen Eltern danken, die mich stets unterstützt haben und mir das Studium erst ermöglichten. 



\section{Lebenslauf}

Persönliche Daten

Name:

Geburtsdaten:

Staatsangehörigkeit:

Schulbildung

1982-1986

1986-1995

1.07.1995

Wehrdienst

1995-1996

Studium

1996-1998

16.10 .1998

1998-2001

2000-2001

8.11.2001

Promotion

seit 2002
Thorsten Scharf

18. Oktober 1975 in Dinkelsbühl

deutsch

Mittelpunkt Grundschule (heute Kugelsburgschule) in Volkmarsen

Christian Rauch Schule in Arolsen (heute Bad Arolsen), allgemeine Hochschulreife

Wehrdienst Frankenberg/Eder

Grundstudium der Physik an der Georg-August-Universität Göttingen Vordiplomprüfung Physik

Hauptstudium der Physik an der Georg-August-Universität Göttingen Diplomarbeit in der Arbeitsgruppe von Prof. Dr. H.-U. Krebs am Institut für Materialphysik.

Diplom, Titel der Arbeit: Mechanische Spannungen und Struktur laserdeponierter metallischer Filme in Abhängigkeit von der Teilchenenergie

Doktorand in der Arbeitsgruppe von Prof. Dr. H.-U. Krebs am Institut für Materialphysik. 